\title{
Cellular responses mediated by the transcription factor STAT1 in murine inflammatory diseases
}

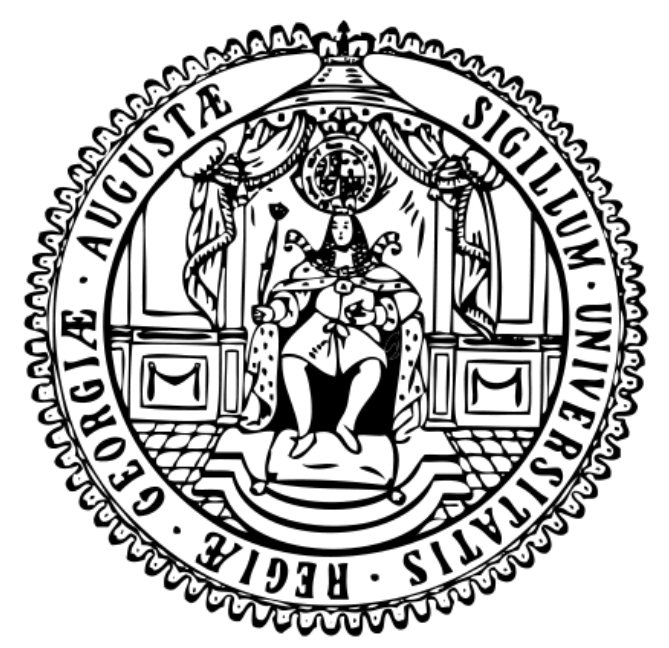

Dissertation

In partial fulfilment of the requirements for the degree

"Doctor rerum naturalium (Dr. rer. nat.)"

of the Georg-August-Universität Göttingen

within the "Molecular Medicine" study program

of the Georg-August University School of Science (GAUSS)

submitted by

Theresa Riebeling

born in Schwalmstadt

Göttingen 2016 


\section{Members of the thesis committee:}

Prof. Dr. mult. Thomas Meyer (supervisor)

Email: $\quad$ thomas.meyer@med.uni-goettingen.de

Postal Adress: $\quad$ Institute of Psychosomatic Medicine and Psychotherapy University Medical Centre Göttingen

Georg-August-University Göttingen

Waldweg 33

37073 Göttingen

Prof. Dr. rer. nat. Susanne Lutz (second member of the thesis committee)

Email: $\quad$ susanne.lutz@med.uni-goettingen.de

Postal Adress: $\quad$ Institute of Pharmacology and Toxicology

University Medical Centre Göttingen

Georg-August-University Göttingen

Robert-Koch-Straße 40

37075 Göttingen

Prof. Dr. med. Dörthe Katschinski (third member of the thesis committee)

Email: _ doerthe.katschinski@med.uni-goettingen.de

Postal Adress: $\quad$ Institute of Cardiovascular Physiology

University Medical Centre Göttingen

Georg-August-University Göttingen

Humboldtallee 23

37073 Göttingen

Date of Disputation: 27 October 2016 


\section{AFFIDAVIT}

Here I declare that my doctoral thesis entitled

"Cellular responses mediated by the transcription factor STAT1 in murine inflammatory diseases"

has been written independently with no other sources and aids than quoted.

Theresa Riebeling

Göttingen, September 2016 


\section{List of publications}

Riebeling T, Staab J, Herrmann-Lingen C, Meyer T. DNA binding reduces the dissociation rate of STAT1 dimers and impairs the interdimeric exchange of protomers. BMC Biochem 2014;15:28.

Staab J, Riebeling T, Koch V, Herrmann-Lingen C, Meyer T. The two interfaces of the STAT1 N-terminus exhibit opposite functions in IFN $\gamma$-regulated gene expression. Mol Immunol 2015;67(2 Pt B):596-606. 


\section{Table of contents}

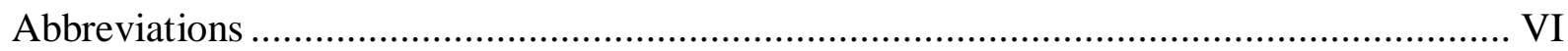

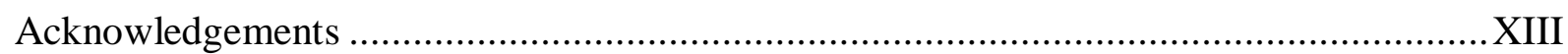

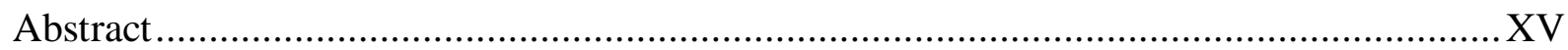

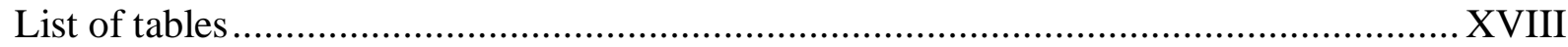

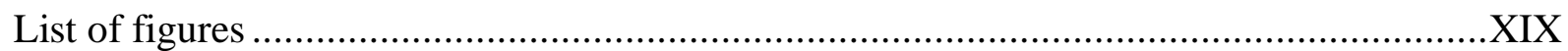

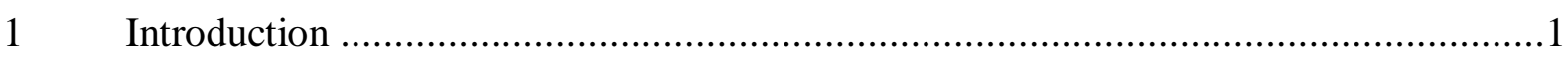

1.1 STAT1 - A key transcription factor in immunity and inflammation .........................1

1.2 JAK/STAT signalling, the actin cytoskeleton and the ERM-proteins .......................11

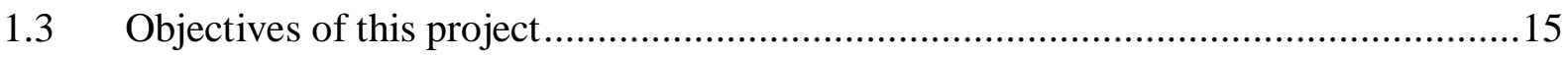

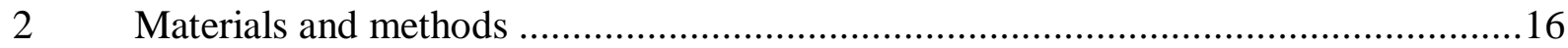

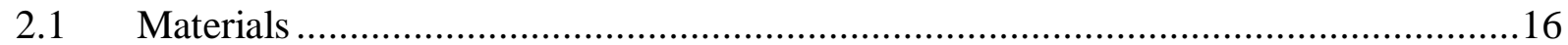

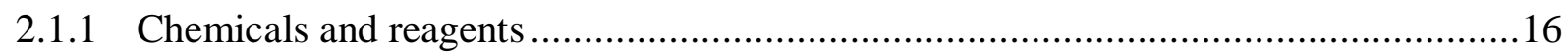

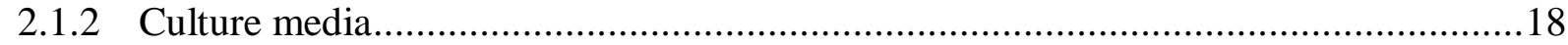

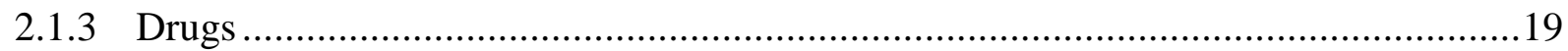

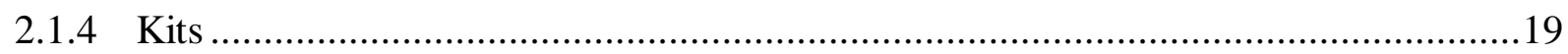

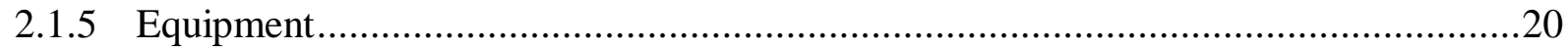

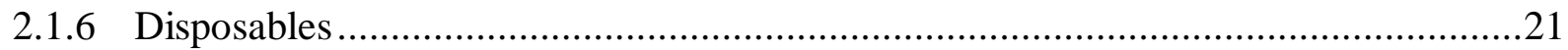

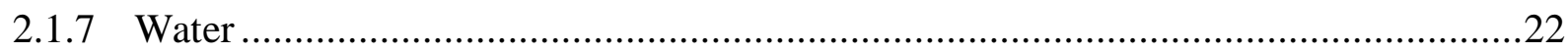

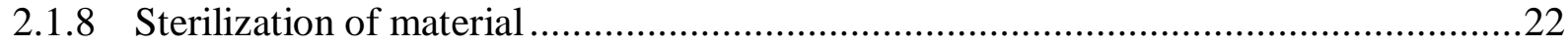

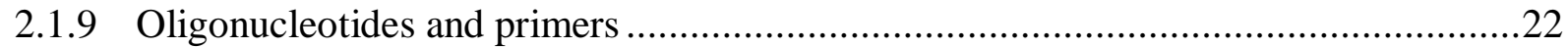

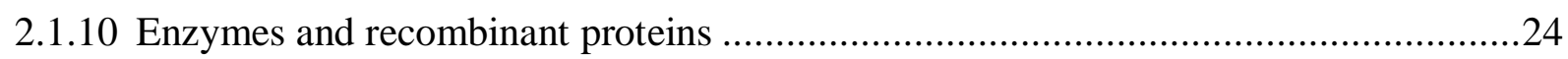

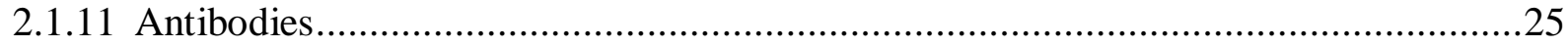

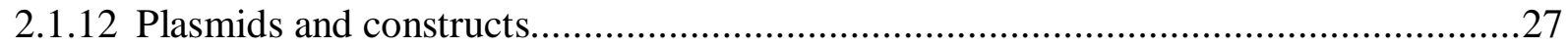

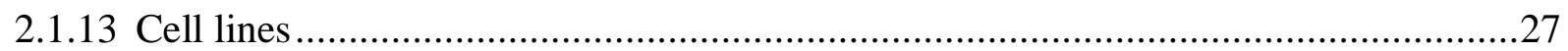

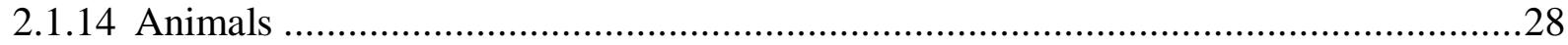

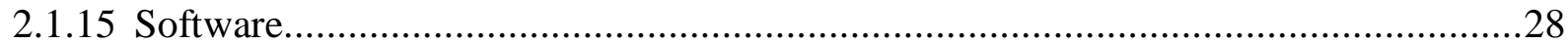

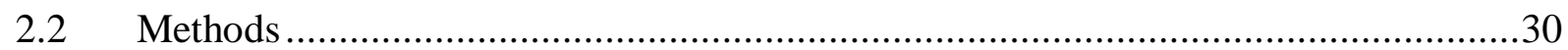

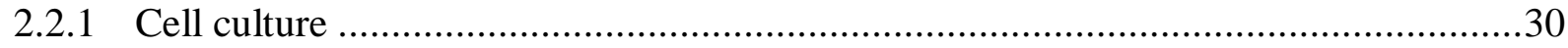

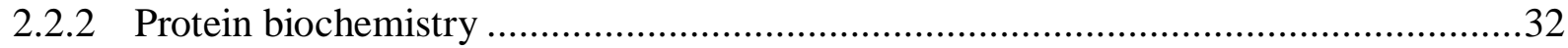

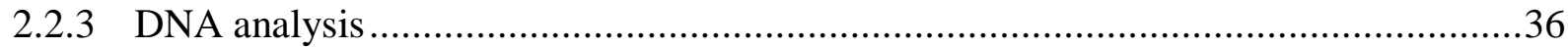




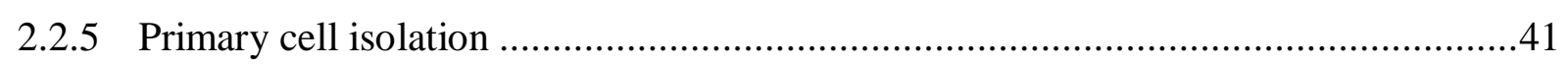

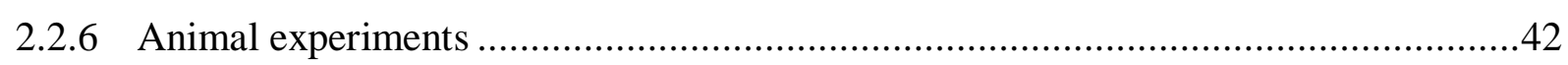

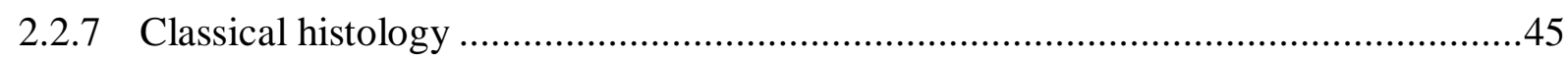

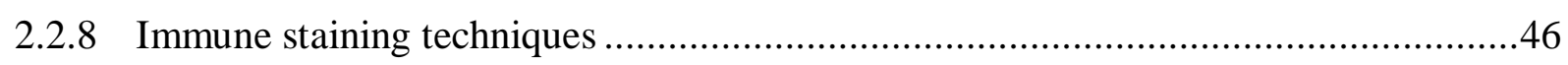

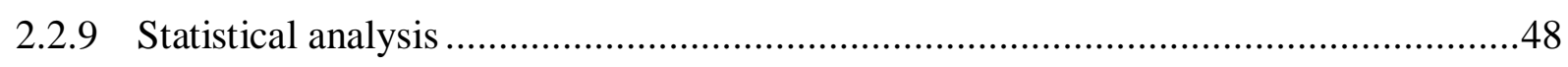

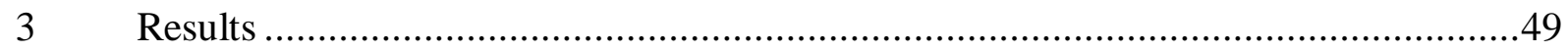

3.1 N-terminus-mediated intradimeric interactions are dispensable for protomer exchange

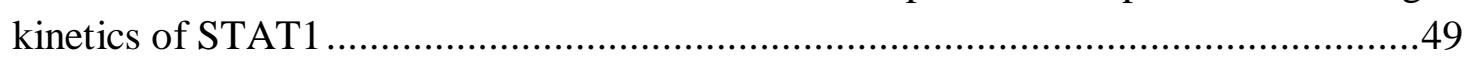

3.1.1 DNA-binding kinetics do not differ among native STAT1 and GFP-tagged STAT1 ..50

3.1.2 Native and GFP-tagged STAT1 form hetero-tetrameric complexes ex vivo................52

3.1.3 The rate of protomer exchange is reduced through binding to DNA...........................53

3.1.4 The exchange of STAT1 protomers is a process independent of intradimeric N-

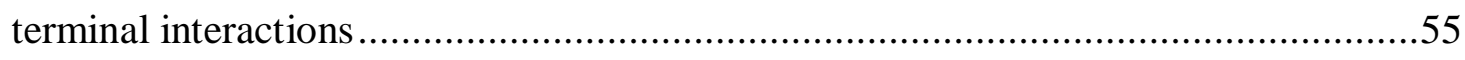

3.2 Gene expression of ezrin is weakly induced by STAT1 ......................................59

3.2.1 Unique GAS motif in the promoter region of the human ezrin gene was identified ....59

3.2.2 STAT1 readily binds to the tandem GAS motif as a dimer but poorly as a tetramer ...60

3.2.3 STAT1 associates to the EZR promoter in Jurkat cells .........................................62

3.2.4 IFN $\gamma$-activated STAT1 induces EZR gene transcription .....................................63

3.2.5 No evidence of IFN $\gamma$-induced ezrin expression in leukemia cell lines ......................65

3.2.6 Ex vivo analysis of STAT1-deficient and STAT1-F77A mutant mice does not show decreased ezrin protein expression ..............................................................6

3.3 Assessment of the role of IFN $\gamma$-dependent STAT1 signalling in myocardial infarction

3.3.1 General characteristics of the study cohort and infarct survival ............................ 71

3.3.2 H\&E staining did not reveal differences in the size of the infarcted area...................73

3.3.3 Mice carrying the STAT1-F77A mutant are protected from adverse cardiac remodelling......

3.3.4 STAT1 expression is reduced in hearts of STAT1-F77A knockin mice compared with the wildtype on day 3 following myocardial infarction .................................... 80

3.3.5 Immune histochemistry shows an accumulation of cells expressing tyrosinephosphorylated STAT1 in the inflammatory infiltrate.

3.3.6 No evidence of altered recruitment of neutrophils, monocytes or T cells to areas of myocardial infarction was observed in STAT1-F77A mutant mice. 
3.4 STAT1 deficiency delays onset of EAE upon challenge with lipopolysaccharide ......88

3.4.1 STAT1 knockout mice are protected upon LPS challenge ....................................89

3.4.2 Onset of clinical symptoms of EAE is delayed in STAT1 knockout mice injected with

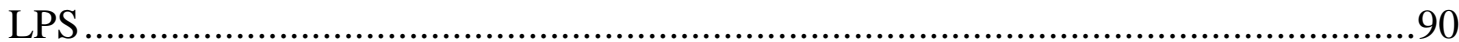

3.4.3 Cells from STAT1 knockout mice show increased proliferation upon $\mathrm{MOG}_{35-55}$

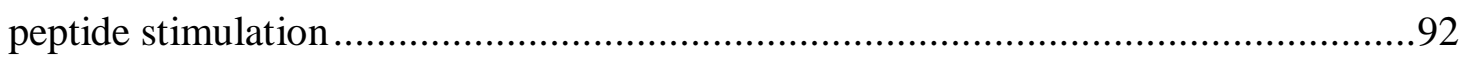

3.4.4 STAT1 knockout cells produce increased amounts of IL-17A and IFN $\gamma \ldots \ldots \ldots \ldots \ldots . . . . .94$

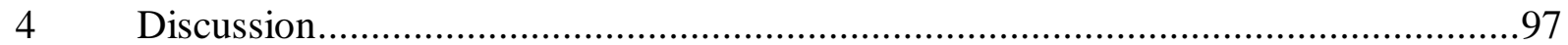

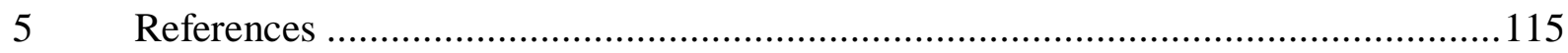

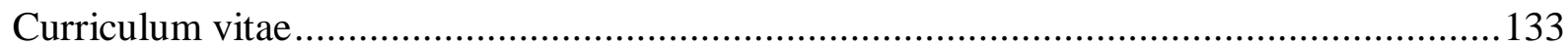




\section{Abbreviations}

A

AF

APC

APCs

APS

ATP

BDGP

BSA

C

C3a

CBP/p300

CCL

CCR2

CD

cDNA

CFA

ChIP

CIS

CNS

Comp

Con A

CREB

CXCL

Cy

DAB

DMEM

DMSO

DNA

dNTP

DTT

EAE
Adenine

Alexa Fluor dye

Allophycocyanin

Antigen-presenting cells

Ammonium persulphate

Adenosine triphosphate

Berkeley drosophila genome project

Bovine serum albumin

Cytosine

Complement C3 isoform a

CREB-binding protein

C-C motif chemokine ligand

C-C motif chemokine receptor type 2

Cluster of differentiation

Complementary deoxyribonucleic acid

Complete Freund's adjuvant

Chromatin immunoprecipitation

Cytokine-inducible $\mathrm{SH} 2$-domain containing protein

Central nervous system

Competition

Concanavalin A

cAMP response element-binding protein

$\mathrm{C}-\mathrm{X}-\mathrm{C}$ motif chemokine ligand

Cyanine

3,3'-diaminobenzidine

Dulbecco's modified Eagle's medium

Dimethyl sulphoxide

Deoxyribonucleic acid

Deoxynucleotide triphosphates

Dithiothreitol

Experimental autoimmune encephalomyelitis 
EDTA

EF

EGF

EGTA

EH medium

ELISA

EMSA

ERM

EZR

FACS

F-actin

FAS

FBS

FERM

FITC

FOXP3

FS

G

GAPDH

GAS

GBP

GFP

GH

GTP

H\&E

HEPES

HMGB1

HPV

HSP

i.p.

ICAM

IFIT1

IFN
Ehylenediaminetetraacetic acid

Ejection fraction

Epidermal growth factor

Ethylene glycol-bis( $\beta$-amimoethyl ether)-N,N,N',N'-tetraacetic acid

Preservation medium

Enzyme-linked immunosorbent assay

Electrophoretic mobility shift assay

Ezrin, radixin, moesin

Ezrin

Fluorescence-activated cell sorting

Filamentous actin

Fractional area shortening

Fetal bovine serum

Four-point-one, ezrin, radixin, moesin

Fluorescein isothiocyanate

Forkhead box P3

Fractional shortening

Guanine

Glyceraldehyde 3-phosphate dehydrogenase

Interferon- $\gamma$ activated site/sequence

Guanylate binding protein

Green fluorescent protein

Growth hormone

Guanosine triphosphate

Haemalum and eosin

4-(2-hydroxyethyl)-1-piperazineethanesulphonic acid

High mobility group box 1

Human papilloma virus

Heat-shock protein

Intraperitoneal

Intercellular adhesion molecule

Interferon-induced protein with tetratricopeptide repeats 1

Interferon 


\begin{tabular}{|c|c|}
\hline IFNAR & Interferon- $\alpha$-receptor \\
\hline IFNGR & Interferon- $\gamma$-receptor \\
\hline IgE & Immunoglobulin $\mathrm{E}$ \\
\hline IGF & Insulin-like growth factor \\
\hline IgG & Immunoglobulin $\mathrm{G}$ \\
\hline IL & Interleukin \\
\hline iNOS & Inducible nitric oxide synthase \\
\hline IRAK & Interleukin-1 receptor associated kinase \\
\hline IRF & Interferon-regulatory factor \\
\hline ISGF & Interferon-stimulated gene factor \\
\hline ISRE & IFN $\alpha$-stimulated gene response element \\
\hline JAK & Janus kinase \\
\hline KO & Knockout \\
\hline LIF & Leukemia-inhibitory factor \\
\hline $\mathbf{L N}$ & Lymph node \\
\hline LPS & Lipopolysaccharide \\
\hline L-selectin & Lymphocyte selectin \\
\hline $\mathbf{L V}$ & Left ventricle \\
\hline Ly49A & Killer cell lectin-like receptor, subfamily A, member 1 \\
\hline Ly-6C / Ly-6G & Lymphocyte antigen 6 complex, locus $\mathrm{C} / \mathrm{G}$ \\
\hline LY96 & Lymphocyte antigen 96 \\
\hline MHC & Major histocompatibility complex \\
\hline MI & Myocardial infarction \\
\hline MOG $_{35-55}$ & Myelin oligodendrocyte glycoprotein peptide, amino acids 35-55 \\
\hline MPO & Myeloperoxidase \\
\hline MS & Multiple sclerosis \\
\hline Mx-Cre & Cre-recombinase expressed under the control of the MX dynamin- \\
\hline & like GTPase 1 \\
\hline MyD88 & Myeloid differentiation primary response gene 88 \\
\hline n.d. & Not detectable \\
\hline NEAA & Non-essential amino acid \\
\hline NFкB & Nuclear factor $\kappa B$ \\
\hline NK & Natural killer \\
\hline NOS & Nitric oxide synthase \\
\hline
\end{tabular}




\begin{tabular}{|c|c|}
\hline N-terminus & Amino-terminus \\
\hline PAGE & Polyacrylamide gel electrophoresis \\
\hline PBS & Phosphate-buffered saline \\
\hline PCR & Polymerase chain reaction \\
\hline PE & Phycoerythrin \\
\hline p-ERM & Phosphorylated ERM protein \\
\hline PI3K & Phosphatidylinositol-4,5-biphosphate 3-kinase \\
\hline PIAS & Protein inhibitor of activated STATs \\
\hline PKC & Protein kinase $\mathrm{C}$ \\
\hline Poly-dIdC & Poly-deoxyinosinic-deoxycytidylic acid \\
\hline p-STAT & Phosphorylated STAT protein \\
\hline PTP1B & Protein tyrosine phosphatase 1B \\
\hline PVDF & Polyvinylidene fluoride \\
\hline qPCR & Quantitative polymerase chain reaction \\
\hline Rac1 & RAS-related C3 botulinum substrate 1 \\
\hline RAGE & Receptor of advanced glycation end products \\
\hline Rap1 & RAS-related protein 1 \\
\hline RAR & Retinoic acid receptor \\
\hline ReMed & Restimulation medium \\
\hline Rho & Ras homolog \\
\hline RIPA & Radioimmunoprecipitation assay \\
\hline RNA & Ribonucleic acid \\
\hline ROCK & Rho associated coiled-coil containing protein kinase \\
\hline ROR $\gamma \mathbf{t}$ & RAR-related orphan receptor $\gamma \mathrm{t}$ \\
\hline ROS & Reactive oxygen species \\
\hline RPMI & Roswell Park Memorial Institute medium \\
\hline $\mathbf{R V}$ & Right ventricle \\
\hline SDS & Sodium dodecyl sulphate \\
\hline SH2 & Src-homology \\
\hline SHP & SH2-containing phosphatase \\
\hline SLC9A1 & Solute carrier family 9 member A1 \\
\hline SOCS & Suppressor of cytokine signalling \\
\hline STAT & Signal transducer and activator of transcription \\
\hline $\mathbf{T}$ & Thymine \\
\hline
\end{tabular}


TBE

T-bet

TBS(T)

TBX21

TC

TCR

TE buffer

TEMED

TGF

Th

TLR

TMB

TNF

TRAF6

TSS

TYK

Untr

UV

VCAM

Vol $_{\mathbf{d}}$

Vols $_{\text {s }}$

WT

ZO

$\alpha \mathrm{S1} / \alpha \mathrm{S3}$
Tris, boric acid, EDTA buffer

T cell-specific T-box transcription factor

Tris buffered saline (supplemented with Tween-20)

T-box 21

Tyrosine phosphatase

$\mathrm{T}$ cell receptor

Tris EDTA buffer

Tetramethylethylenediamine

Transforming growth factor

T helper

Toll-like receptor

3,3',5,5'-tetramethylbenzidine

Tumour necrosis factor

TNF receptor-associated factor 6

Transcription start site

Tyrosine kinase

Untreated

Ultra violet

Vascular cell adhesion molecule

Volume diastole

Volume systole

Wildtype

Zona occludens

Anti-STAT1/3 antibody 


\section{Units}

$\begin{array}{ll}\mathbf{\%} & \text { Percent } \\ { }^{\circ} \mathbf{C} & \text { Degree Celsius } \\ \mathbf{b} & \text { Base } \\ \mathbf{b p m} & \text { Beats per minute } \\ \mathbf{B q} & \text { Becquerel } \\ \mathbf{D a} & \text { Dalton } \\ \mathbf{g} & \text { Gram } \\ \mathbf{G} & \text { Gauge } \\ \mathbf{h} & \text { Hour } \\ \mathbf{l} & \text { Liter } \\ \mathbf{m} & \text { Meter } \\ \mathbf{M} & \text { mol/1 } \\ \mathbf{m i n} & \text { Minute } \\ \mathbf{m o l} & \text { Mole } \\ \mathbf{r p m} & \text { Rounds per minute } \\ \mathbf{s e c} & \text { Second } \\ \mathbf{x g} & \text { Times gravity } \\ \mathbf{\Omega} & \text { Ohm }\end{array}$




\section{Amino Acids}

When referring to amino acids either the three letter code or the one letter code is used.

Amino Acid

Alanine

Arginine

Asparagine

Aspartic acid

Cysteine

Glutamic acid

Glutamine

Glycine

Histidine

Isoleucine

Leucine

Lysine

Methionine

Phenylalanine

Proline

Serine

Threonine

Tryptophan

Tyrosine

Valine
Three letter code

Ala

Arg

Asn

Asp

Cys

Glu

Gln

Gly

His

Ile

Leu

Lys

Met

Phe

Pro

Ser

Thr

Trp

Tyr

Val
One letter code

A

$\mathrm{R}$

$\mathrm{N}$

D

C

E

Q

G

H

I

L

K

M

F

P

S

$\mathrm{T}$

W

$\mathrm{Y}$

V 


\section{Acknowledgements}

Now, at the end of my time as a doctorate student, there are a couple of people whom I would like to thank for their help and support during the past three-and-a-half years. Without these people, this piece of work would not have been possible.

I hold the deepest gratitude towards my supervisor Professor Thomas Meyer. Throughout the time of my doctorate he gave me the greatest support and contributed to this project with discussion, advice, ideas, and material. He markedly shaped my view on what it means to be a scientist. I truly admire his dedication and enthusiasm.

Moreover, I would like to thank the members of my thesis committee, Professor Dörthe Katschinski and Professor Susanne Lutz, for their input, help, ideas and support - and for joining my committee while I was already in the middle of my doctorate. I thank Professor Ali El-Armouche who helped to establish the myocardial infarction project during the early phase but then had to leave my thesis committee when he moved to his new position in Dresden. Furthermore, I thank Professor Uwe-Karsten Hanisch who helped in laying the foundation of this project. His early passing was a great loss.

I am grateful to Dr Aleksandar Ivetic, our partner within the IRTG 1816, his lab group, and the entire department of Cardiology at King's College in London for offering me the opportunity to stay, work and learn with and from them during a period of two months last year.

I would like to acknowledge the service unit of the SFB 1002. I thank Sabrina Wollborn and Sarah Zafar for doing a wonderful job in performing the myocardial infarction surgery with great care and skill, Roland Blume and Marcel Zoremba for providing me with clear echocardiographic images and Beate Knocke for teaching me how to evaluate them properly.

Moreover, I want to express my gratitude towards the entire lab group of Professor Alexander Flügel, especially towards Dr Fred Lühder for his help and support in getting the EAE project started, and the technicians Martina Weig, Adriane Stas, and Birgit Curdt for teaching me and helping me with the needed experimental techniques.

I would like to express my gratitude towards my co-workers at the lab of molecular psychocardiology here in Göttingen for being supportive and helpful during the past three years. I hold a deep gratitude towards Dr Julia Staab, our post-doc for her help, ideas and valued discussion, for sharing her experience and expertise, helping me to calm down when I 
was worried and for performing the qPCR on the STAT1 F77A knockin model. I want to thank Asma Doudin for double-checking my results and wish her all the best for her doctorate. Furthermore, I thank our technicians Heike Hühn and Anke Gregus for their excellent technical support and maintenance of the lab.

During my doctorate, I was granted the pleasure of meeting several MD students and rotation students and was allowed to supervise a few bachelor theses. I want to thank all of them for providing a pleasant working atmosphere and bringing new ideas into the lab. There are two students who I want to mention especially: Johannes Wolff for acquiring the proliferation and ELISA data of the EAE experiments with me during his bachelor thesis and Priyanka Menon for immune staining a part of the paraffin sections shown in this thesis. It was a pleasure to work with and supervise them.

Special thanks go to the organizing team of the International Research Training Group (IRTG) 1816. Not only did they help me and my fellow students of the program with funding issues but also supported us by offering workshops, meetings, and seminars and by encouraging us to widen our scope beyond our projects. I thank Dr Christina Würtz, Katja ElArmouche, Fulya Ören, Gry Dihazi, and Ulrike Fischer for the terrific work they have done and for always having an open ear when problems occurred. Furthermore, I want to mention my fellow students of the IRTG 1816 program. I hope that we will keep in touch.

During the past three-and-a-half years I have experienced and received lots of support by people from different departments of the University Medical Centre in Göttingen. I want to thank everybody who helped me at some point of my thesis by providing me with some reagent, a protocol, a cell line, an idea, discussion or technical assistance. I am sorry that there is not enough room to mention all of these people.

Finally, I want to mention my sister Katharina and my aunt Eva for proof-reading this thesis. I thank my parents, family, friends and my fiancé for being supportive, encouraging me to do a doctorate, and for their love, help and care at all times. 


\section{Abstract}

The intracellular transduction of interferon signals from the plasma membrane to the nucleus is mediated by signal transducer and activator of transcription 1 (STAT1) which acts in tetrameric form as a transcription factor in immune responses. In this project, the protomer exchange between STAT1 dimers was studied under kinetic aspects and this process was identified as a potential rate-limiting step in the STAT1 activation/inactivation cycle. The data support an alternative mechanism for the transition between the parallel and anti-parallel conformation of STAT1 dimers based on the dissociation and subsequent reassociation of protomers which does not require reciprocal interactions within the $\mathrm{N}$-terminal domain dimer to stabilize an intermediate conformational state. However, binding to high-affinity STATspecific target sequences, termed gamma-activated sites (GAS), critically interfered with the exchange dynamics. A tandem GAS element in the promoter of the human gene coding for the cytoskeletal protein ezrin was identified in silico as a putative STAT1 target sequence, and was confirmed to bind dimeric STAT1 on each motif to moderately induce gene transcription. However, mice carrying either an N-terminal substitution mutation of STAT1 affecting cooperative DNA binding or a complete functional knockout of the Stat1 gene did not present with altered expression of ezrin and moesin in bone marrow cells compared with mice expressing the wildtype molecule. In a model of myocardial infarction performed by ligation of the left anterior descending coronary artery, male mice expressing the IFN $\gamma$-irresponsive STAT1 mutant displayed higher survival rates, whereas females were protected from adverse cardiac remodelling in the early remodelling phase. The inflammatory infiltrate showed a mild increase in tyrosine-phosphorylated STAT1, while the pool of total STAT1 was significantly reduced in the infarcted areas of knockin animals as compared to their wildtype littermates. In experimental autoimmune encephalomyelitis used as a model for a $\mathrm{T}$ helper cell-induced autoimmune disease, cells obtained from lymphatic organs of STAT1-deficient animals at the time of disease onset were hyperproliferative and secreted high amounts of IFN $\gamma$ and IL-17A. Injection of these mice with lipopolysaccharide during the induction phase of experimental autoimmune encephalomyelitis completely abolished this proliferative phenotype. In summary, the findings demonstrate the role of cooperative DNA binding and tetramer formation of STAT1 in orchestrating complex immunological processes, even in the absence of infectious agents and, furthermore, highlight the function of tyrosinephosphorylated STAT1 as a key factor facilitating the cross-talk between innate and adaptive immunity. 


\section{STAT1-vermittelte zelluläre Antworten in murinen entzündlichen}

\section{Erkrankungen}

Die intrazelluläre Weiterleitung von Interferonsignalen von der Zytoplasmamembran zum Zellkern wird vermittelt über den Signaltransduktor und Aktivator der Transkription 1 (STAT1), welcher in seiner tetrameren Form als Transkriptionsfaktor an Immunantworten beteiligt ist. In diesem Projekt wurde der Protomerenaustausch zwischen STAT1-Dimeren unter kinetischen Gesichtspunkten untersucht und dabei dieser Prozess als ein potentiell geschwindigkeitsbestimmender Schritt des Aktivierungs-/Inaktivierungs-Zyklus von STAT1 identifiziert. Die Daten unterstützen einen alternativen Mechanismus für den Wechsel zwischen der parallelen und antiparallelen Konformation von STAT1-Dimeren basierend auf der Dissoziation und nachfolgenden Reassoziation von Protomeren, bei dem reziproke Interaktionen innerhalb des N-terminalen Domänendimers zur Stabilisierung eines intermediären Konformationsübergangs nicht benötigt werden. Durch Bindung an spezifische DNA-Zielbereiche, als Gamma-aktivierte Sequenzen (GAS) bezeichnet, wird die Dynamik des Protomerenaustauschs wesentlich beeinträchtigt. In der Sequenz des für das zytoskelettale Strukturprotein Ezrin kodierenden humanen EZR-Gens wurde mittels in silico Analyse ein doppeltes GAS-Motiv als mögliche STAT1-Zielsequenz identifiziert und die Bindung von STAT1-Dimeren an jedes der beiden Elemente sowie eine moderate Geninduktion bestätigt. Allerdings zeigen Mäuse mit einer N-terminalen Substitutionsmutation von STAT1, welche die kooperative DNA-Bindung beeinträchtigt, sowie auch ein kompletter funktioneller Knockout des Statl-Gens keine veränderte Expression von Ezrin und Moesin in Knochenmarkszellen verglichen mit Mäusen, die das Wildtyp-Molekül exprimieren. In einem Myokardinfarktmodell durch Ligatur des Ramus interventricularis anterior zeigen männliche Mäuse mit Expression der Interferon- $\gamma$-irresponsiven STAT1-Mutante höhere Überlebensraten, während weibliche Tiere vor den nachteiligen Effekten des kardialen Remodellings in der frühen Phase geschützt sind. In entzündlichen myokardialen Infiltraten dieser Tiere wurde ein geringfügig höheres Expressionsniveau an tyrosinphosphoryliertem STAT1 nachgewiesen, während die Gesamtproteinmenge an STAT1 gegenüber dem Wildtyp reduziert war. Zellen aus lymphatischen Organen STAT1-defizienter Tiere mit experimenteller autoimmuner Enzephalomyelitis, die als Modell einer T-Helfer-Zellvermittelten Autoimmunerkrankung verwendet wurde, zeigten einen hyperproliferativen Phänotyp und sezernierten größere Mengen an IFN $\gamma$ und IL-17A. Injektion dieser Mäuse mit Lipopolysaccharid während der Induktionsphase der experimentellen autoimmunen 
Enzephalomyelitis hob den hyperproliferativen Phänotyp vollständig auf. Zusammenfassend demonstrieren die Ergebnisse aus dieser Arbeit die Bedeutung einer kooperativen DNABindung und Tetramerstabilisierung von STAT1 im Zusammenspiel komplexer immunologischer Prozesse auch in Abwesenheit infektiöser Pathogene und unterstreichen zudem die Schlüsselrolle von tyrosinphosphoryliertem STAT1 bei der Verknüpfung zwischen angeborenem und erworbenem Immunsystem. 


\section{List of tables}

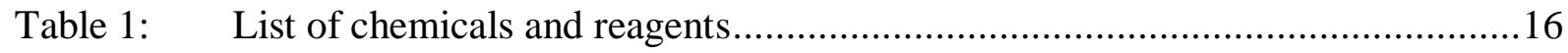

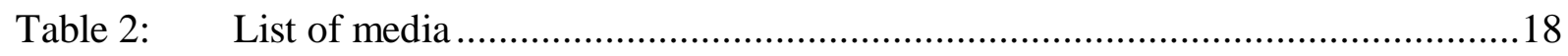

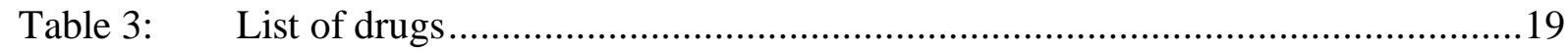

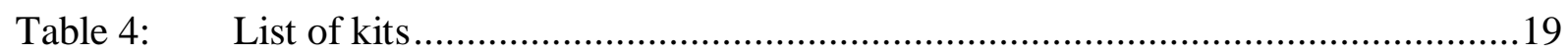

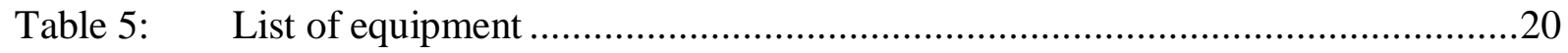

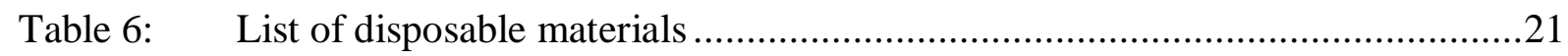

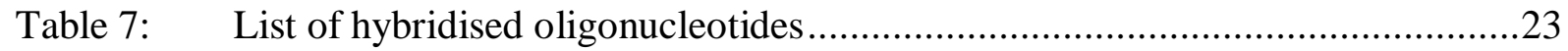

Table 8: List of oligonucleotide primers for genotyping ….....................................23

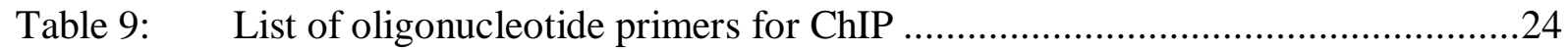

Table 10: List of oligonucleotide primers for quantitative PCR (qPCR) ........................24

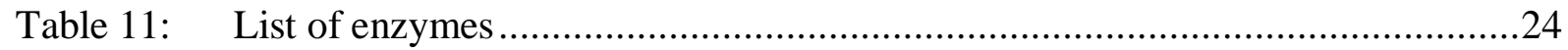

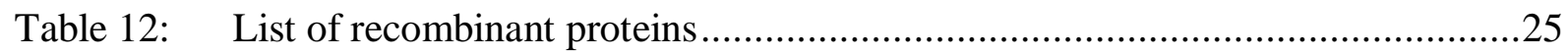

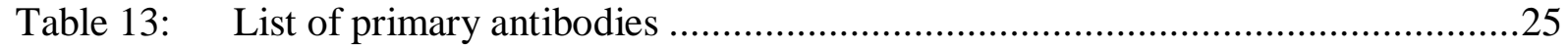

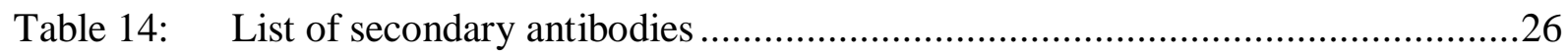

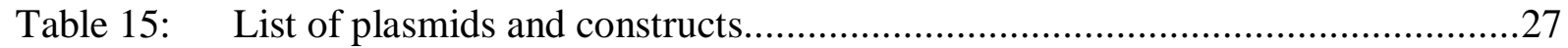

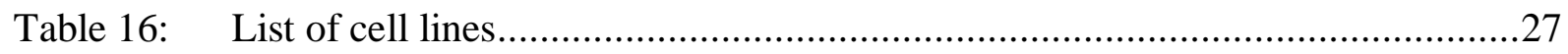

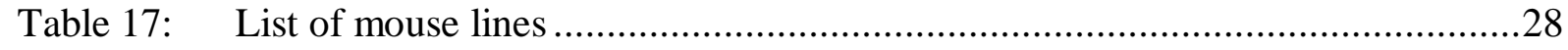

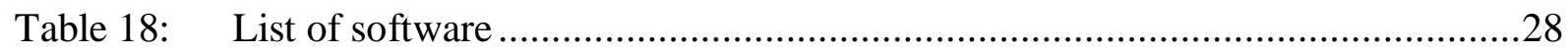

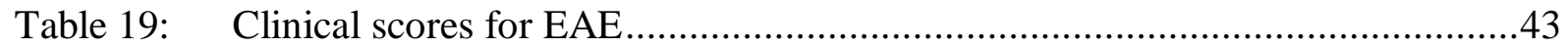

Table 20: Endpoint calculation of echocardiography data ..........................................45

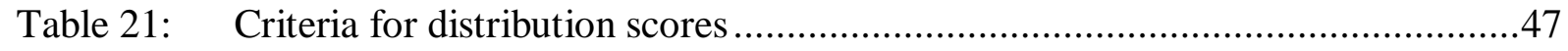

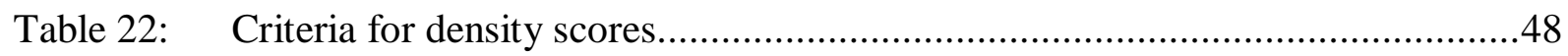

Table 23: GAS motifs surrounding the transcription-start site (TSS) in the promoter of the

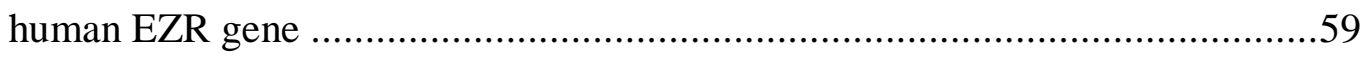

Table 24: GAS motifs surrounding the TSS of the murine EZR gene sequence ................60

Table 25: Survival rates of mice after undergoing myocardial infarction surgery at day 3 post-myocardial infarction 


\section{List of figures}

Figure 1: $\quad$ Model of tetramer stabilization of STAT1 binding to DNA ................................

Figure 2: $\quad$ Proposed mechanisms for STAT1 conformational change ..............................49

Figure 3: Native STAT1 and STAT1-GFP both favour tetrameric binding to tandem GAS sites

Figure 4: Competition assay showing the dissociation kinetics of STAT1 dimers from single GAS elements

Figure 5: Native STAT1 and STAT1-GFP can form hetero-tetramers

Figure 6: STAT1 hetero-tetramers consisting of two homodimers are formed on tandem GAS sites

Figure 7: Untagged and GFP-tagged STAT1 form hetero-tetramers with 3:1 and 1:3 stoichiometry when unbound to DNA

Figure 8: Binding of STAT1 to DNA hinders the exchange of protomers

Figure 9: Protomer exchange kinetics of dimers does not differ between the wildtype STAT1 and the F77A mutant

Figure 10: Amino-terminal interactions are dispensable for monomer exchange of STAT1

Figure 11: EMSA showing STAT1 binding to tandem GAS elements in the human EZR promoter .....

Figure 12: ChIP analysis of STAT1 association to the EZR promoter in IFN $\gamma$-treated Jurkat cells

Figure 13: Real-time PCR results from Jurkat cells and HL-60 cells treated with IFN $\gamma$ for $2 \mathrm{~h}, 6 \mathrm{~h}$, and $10 \mathrm{~h}$, respectively

Figure 14: Real-time PCR results of Jurkat cells treated with IFN $\alpha$ and IFN $\gamma$ showing induction of the EZR gene

Figure 15: Expression of ezrin and moesin in Jurkat and HL-60 cells treated with IFN $\alpha$ or IFN $\gamma$

Figure 16: Expression and phosphorylation of ezrin and moesin in HL-60 cells treated with IFN $\alpha$ or IFN $\gamma$.

Figure 17: Ex vivo analysis of baseline expression of ezrin and moesin in bone marrow cells from wildtype, STAT1-deficient and STAT1-F77A mice

Figure 18: Phosphorylation levels of ezrin and moesin in bone marrow from wildtype, STAT1-deficient and STAT1-F77A knockin mice treated ex vivo with interferons.

Figure 19: Kaplan-Meier survival analysis of mice subjected to experimental myocardial infarction

Figure 20: H\&E staining of acute myocardial infarction 
Figure 21: Evaluation of the extent of inflammation according to H\&E staining of sections of paraffin-embedded cardiac tissue three days after myocardial infarction ......75

Figure 22: Increase in left ventricular diameter after myocardial infarction ......................76

Figure 23: Increased left ventricular cross-sectional area due to myocardial infarction ......77

Figure 24: Increase in end-diastolic and end-systolic volume after myocardial infarction ..78

Figure 25: The reduced cardiac function after myocardial infarction is blunted in mice with impaired STAT1 tetramer stabilization .......................................................... 79

Figure 26: Expression and phosphorylation of STAT1 and STAT3 in heart tissue from STAT1-F77A knockin mice on day 3 after myocardial infarction.

Figure 27: Staining of tyrosine-phosphorylated STAT1 in heart sections on day 3 after myocardial infarction or sham operation

Figure 28: Analysis of the relative numbers of regulatory $\mathrm{T}$ cells in lymph node and spleen of the STAT1-F77A mouse line

Figure 29: Reduced activation of two known IFN $\gamma$-target genes by STAT1-F77A in reconstituted U3A cells

Figure 30: Staining of myeloperoxidase (MPO) on day 3 following myocardial infarction or sham operation

Figure 31: Staining of CD68 following ligation of the left ascending coronary artery or sham operation on day 3

Figure 32: Expression of CD3 in infarcted heart tissue on day 3 follow-up.......................88

Figure 33: Survival before onset of EAE upon LPS challenge

Figure 34: Clinical scores in STAT1-deficient and wildtype mice treated with and without

LPS injection

Figure 35: Mean date of EAE onset.

Figure 36: Relative proliferation rates of cells derived from lymphatic organs challenged with $\mathrm{MOG}_{35-55}$ peptide or concanavalin A

Figure 37: IL-17A concentrations in the supernatants of spleen and lymph node cells from $\mathrm{MOG}_{35-55}$-immunised mice in the presence or absence of LPS challenge.

Figure 38: Dysregulated IFN $\gamma$ production in the supernatants of primary cells from lymphatic organs of STAT1 knockout animal cells immunized with $\mathrm{MOG}_{35-55}$ peptide in the presence or absence of LPS co-stimulation..... 


\section{Introduction}

\subsection{STAT1 - A key transcription factor in immunity and inflammation}

Signal transducer and activator of transcription 1 (STAT1) belongs to a family of latent signal-dependent transcription factors that is highly conserved among mammals and is involved in a plethora of cellular processes including immunity, cell growth, differentiation and apoptosis (Levy and Darnell, 2002). The protein was first described in the early 1990s as part of a complex that was phosphorylated in response to interferon- $\alpha$ (IFN $\alpha)$ and IFN $\gamma(\mathrm{Fu}$, 1992; Shuai et al., 1992).

STAT family proteins are thought to have evolved with the development of the first multicellular organisms. They were reported to be present in Dictyostelium discoideum and Drosophila melanogaster but were not found in Saccharomyces cerevisiae (Yan et al., 1996; Kawata et al., 1997). In mammals, there are seven different forms of STAT proteins, namely STAT1, STAT2, STAT3, STAT4, STAT5a, STAT5b and STAT6. In mice, the genes for these proteins cluster in three regions: Stat1 and Stat4 at the proximal region of chromosome 1, Stat2 and Stat6 at the distal region of chromosome 10 and Stat3, Stat5a and Stat5b at the distal region of chromosome 11 (Copeland et al., 1995). In humans, the genes encoding STAT1 and STAT4 are localised in a head to tail tandem orientation to the long arm of chromosome 2, STAT3, STAT5 $a$ and STAT5 $b$ are localized to chromosome 17, and STAT2 and STAT6 are both found on chromosome 12 (Darnell, 1997).

Upon activation by extracellular signalling ligands at the plasma membrane, STAT proteins exert their roles as transcription factors in the nucleus and modulate cytokinedependent gene expression (Darnell, 1997). Among the signalling molecules are a variety of cytokines including interferons, interleukins, growth factors, and hormones (Abroun et al., 2015).

The characteristic structure of members of the STAT protein family is a modular set-up of an amino-terminal, a coiled-coil, a DNA-binding, a Src-homology (SH2), and a transactivation domain (from amino- to carboxy-terminus). The molecular weight of mammalian STAT proteins ranges between $80 \mathrm{kDa}$ and $113 \mathrm{kDa}$ (Darnell, 1997; Levy and Darnell, 2002). Conserved among the STAT transactivation domains is a tyrosine residue that is phosphorylated by Janus kinases (JAKs) upon activation. In STAT1, this residue is located at position 701, while the corresponding position in the STAT3 molecule is located at 705 
(Shuai et al., 1993a; Kaptein, 1996). By differential splicing or proteolysis, all STAT proteins except for STAT2 have been found to be expressed with different isoforms of different protein size and slightly different regulation. In the case of STAT1 and STAT3, a full-length $\alpha$ - and a truncated $\beta$-isoform are expressed. Both $\beta$-isoforms lack a serine residue which is conserved among full-length STAT1, STAT3, STAT5a and STAT5b. Phosphorylation of this serine residue has been shown to boost STAT1 transcriptional activity rendering the $\beta$ isoforms less active (Schindler et al., 1992a; Schaefer et al., 1995; Wang et al., 1996; Decker and Kovarik, 2000; Horvath, 2000; Lödige et al., 2005).

The three-dimensional structures of STAT1 and STAT3 dimers bound to DNA were determined using crystallographic analyses. The data revealed the nature of STAT-DNA binding including the dimerization of the two protomers via reciprocal interactions of their SH2 domains and the binding interface interacting with the DNA (Becker et al., 1998; Chen et al., 1998). However, these structural analyses failed to determine the structure of the $\mathrm{N}$ terminal domain and parts of the transactivation domain. Combination of the models of STAT1 and STAT4 for which crystallography of the N-terminus had been successful led to a model that predicted interactions of the N-termini within a dimer (Baden et al., 1998; Vinkemeier et al., 1998; Chen et al., 2003b). Crystallographic analysis of unphosphorylated STAT1 confirmed these predictions based on the hybrid model and showed that unphosphorylated cytosolic STAT1 forms tetrameric complexes as dimers of dimers. These studies identified two different conformational states of STAT1 dimers that would both be supported by N-terminal interactions and showed that phosphorylated STAT1 favoured the more open parallel alignment, while unphosphorylated STAT1 was more likely to acquire the more condensed antiparallel state (Mao et al., 2005; Wenta et al., 2008). STAT1 dimers in the parallel conformation were assumed to interact with the receptor and shown to bind to DNA.

The main role in stabilizing this conformational state was attributed to the $\mathrm{SH} 2$ domain. Stabilization of the antiparallel state is mainly achieved through a combination of $\mathrm{N}$-terminal domain interactions and interactions of the coiled-coil domain with the DNA-binding domain of the partner protomer. It was proposed that phosphorylated STAT1 dimers in the parallel conformation are a poor substrate for phosphatases and cannot leave the nucleus unless they are dephosphorylated (Meyer et al., 2003). This suggests that a conformational shift is a main constituent of the STAT1 activation-inactivation cycle. It was predicted that interactions of the N-termini facilitate the shift from the parallel to the antiparallel conformational state and 
that the phenylalanine at position 77 plays a major role (Mao et al., 2005; Zhong et al., 2005; Mertens et al., 2006; Wenta et al., 2008).

Second to the SH2 domain, the amino-terminal domain is the most highly conserved domain among the STAT proteins. However, domain exchange experiments have shown that it is not interchangeable. In STAT1, the amino-terminal domain spans the first 135 amino acids (Strehlow and Schindler, 1998; Murphy et al., 2000) and through this domain, proteinprotein interactions between two STAT1 molecules are facilitated. Based on structural data and targeted mutagenesis, a conserved phenylalanine residue was identified at position 77 in STAT1 and at position 81 in STAT5 that is essential for the formation of tetramers (John et al., 1999; Meyer et al., 2004). Mutation of this residue in STAT1 to alanine resulted in severely impaired cooperative DNA binding and reduced expression of some but not all STAT1 target genes. Furthermore, the STAT1 N-terminus provides a surface to interact with other modulating factors such as the CREB-binding protein (CBP/p300) and protein inhibitor of activated STAT1 (PIAS1) (Leung et al., 1996; Darnell, 1997; Horvath, 2000; Shuai, 2000). In summary, DNA-bound tetramers and higher-order oligomers of STAT1 are formed by reciprocal amino-terminal interactions.
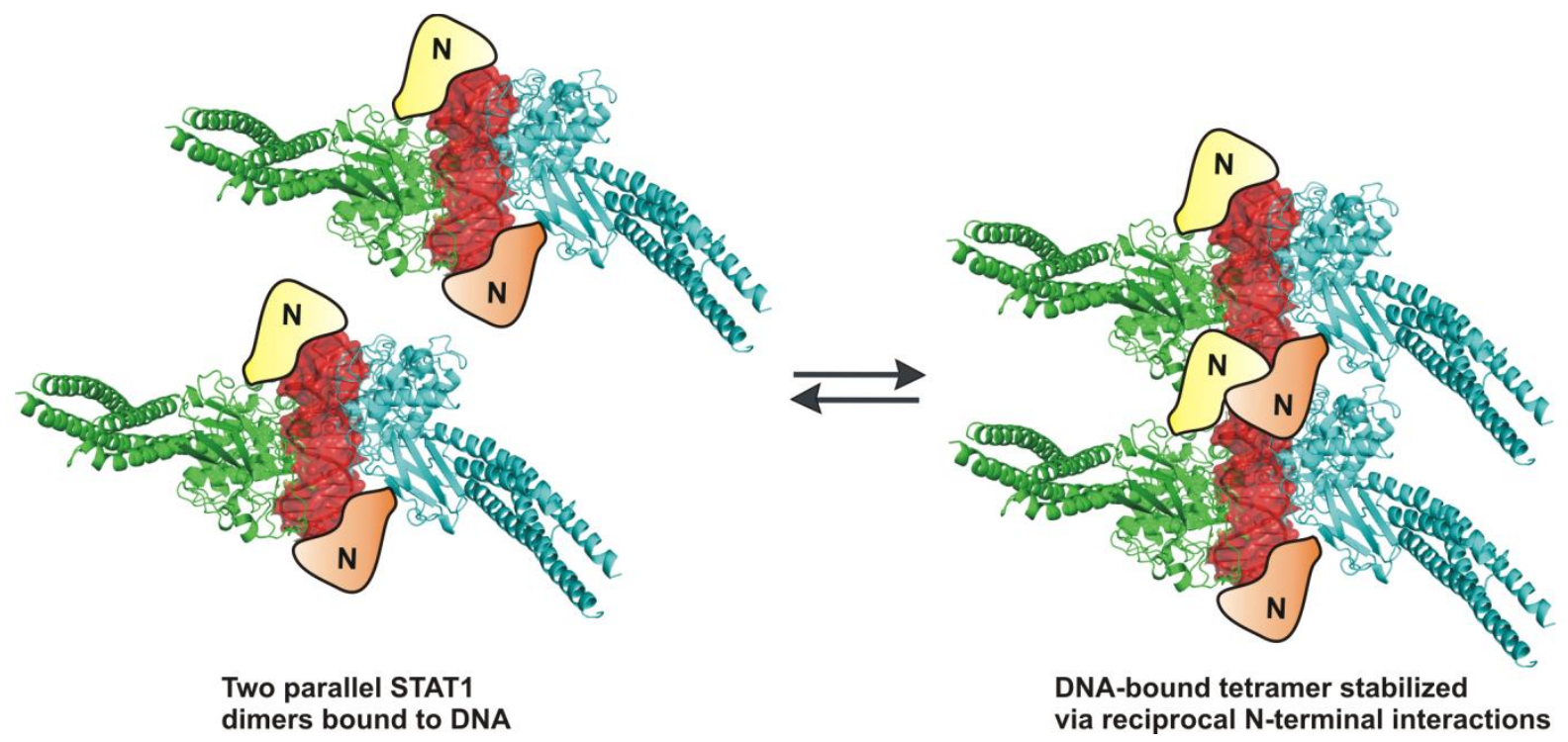

Figure 1: Model of tetramer stabilization of STAT1 binding to DNA. Shown are STAT1 dimers in parallel alignment (blue and green) bound to the DNA double helix (red). The N-termini (N) of two dimers interact to facilitate cooperative DNA binding.

The coiled-coil domain of STAT1 consists of 181 amino acids and forms a structure of four $\alpha$-helices. It contains a leucine-rich nuclear export signal that is pivotal for the STAT1 
activation cycle (Begitt et al., 2000). In addition, the coiled-coil domain forms an interface for interactions with the DNA-binding domain of a partner molecule in order to stabilize the antiparallel dimer conformation. Targeted mutagenesis of the residues Q340, G384, Q408 of the DNA-binding domain and residue F172 disrupts these interactions and led to the destabilization of the antiparallel conformational state and prolonged tyrosine phosphorylation (Begitt et al., 2000; Mao et al., 2005; Mertens et al., 2006).

The DNA-binding domain contains the interface for the interaction of STAT1 with target sequences on DNA. Through several distinct residues, STAT1 interacts with the major as well as the minor groove of the DNA-double helix (Chen et al., 1998). Besides the DNA binding activity, this domain also provides the nuclear localisation sequence needed for the interaction with importin- $\alpha-5$ to facilitate nuclear import of the phosphorylated STAT1 dimer (Fagerlund et al., 2002; McBride et al., 2002; Meyer et al., 2002).

The STAT1 linker domain spans the amino acids 488 to 575 (Chen et al., 1998). A variety of residues have been identified in the linker domain of STAT1 as well as of STAT3 which upon mutation, alter DNA-binding kinetics and affect gene transcription. It is believed that by interactions of the linker domain with the SH2 domain and the DNA-binding domain, the process of association to and release from DNA is regulated (Yang et al., 2002; Hüntelmann et al., 2014; Mertens et al., 2015). Furthermore, the measles virus phosphoprotein was found to interact with the STAT1 linker domain, thereby, preventing JAK1-induced activating phosphorylation, indicating that the linker domain is a target of viral pathogens during immune evasion (Devaux et al., 2013).

The SH2 domain is highly conserved among the STAT family members and provides the surface for the dimerization of tyrosine-phosphorylated STAT1 molecules. For this interaction, the arginine residue at position 602 is most critical since it interacts with the phosphorylated tyrosine residue 701 of the partner protomer to form a parallel STAT1 dimer (Shuai et al., 1993b; Chen et al., 1998; Wenta et al., 2008).

The transactivation domain of STAT1 spans a region of 67 amino acids and holds the critical phosphorylation site at position 701 as well as the serine residue at position 727 that can be phosphorylated to enhance transcription. The truncated STAT1 $\beta$ isoform lacks transcriptional activation, despite the fact that this isoform is able to dimerize, become tyrosine-phosphorylated and bind to DNA. Due to its low levels of conservation among the different STAT1 variants, the transactivation domain is believed to mediate target gene 
specificity (Shuai et al., 1992; Schindler et al., 1992b; Darnell, 1997; Decker and Kovarik, 2000).

STAT1 is activated mainly by interferon signalling. Interferons are classified into three different subgroups. The type I interferons (IFN $\alpha$, IFN $\beta$ and some related molecules) are known to be essential in orchestrating an antiviral defence. The only mammalian type II interferon is IFN $\gamma$. It has been identified as a main actor in antibacterial, -fungal and -parasite immunity but plays only a limited role in antiviral activity. Type III interferons classified as different isoforms of IFN $\lambda$ were discovered in 2003 . They were found to carry out their main tasks in supporting epithelial barrier function and in immunity against some RNA viruses (Kotenko et al., 2003; Sheppard et al., 2003; Zhang et al., 2008; Lazear et al., 2015).

Interferon signalling is initiated by binding of the cytokine to the extracellular domain of its cognate receptor. Type I interferons bind to the interferon- $\alpha$ receptor (IFNAR) which is comprised of two subunits, IFNAR1 and IFNAR2 (Novick et al., 1994; Platanias, 2005). Extracellular IFN $\gamma$ is detected by the interferon- $\gamma$ receptor (IFNGR) (Langer and Pestka, 1988). IFNAR and IFNGR are both class-II cytokine receptors. They contain an extracellular ligand-binding domain and a transmembrane domain which spans the membrane once. Proteins with tyrosine-kinase activity are bound to the intracellular carboxy-terminal domain of interferon receptors (Bach et al., 1997). Upon binding of the ligand to its receptor, the two receptor subunits dimerize. Janus kinases become activated which enables them to recruit and phosphorylate STAT proteins at a conserved tyrosine residue near the carboxy-terminus to induce activation. The Janus kinases are a relatively small family of tyrosine kinases consisting of only four members, namely JAK1, JAK2, JAK3 and TYK2 (tyrosine kinase 2). They are non-covalently bound to cytokine receptors via a four-point-one, ezrin, radixin, moesin (FERM) domain (Yamaoka et al., 2004). To facilitate differential regulation of type I and type II interferon signalling, IFNAR recruits STAT1 as well as STAT2, while IFNGR exclusively recruits STAT1 (Shuai et al., 1993b; Darnell, 1997). In the case of STAT1, the transcription factor is recruited from a pool of monomers and unphosphorylated dimers located in the cytosolic and nuclear compartment of the resting cell (Yang and Stark, 2008).

Following phosphorylation of STAT1 and STAT2, two different complexes are formed. The STAT1 homodimer is formed by interactions of the SH2 domains of two phosphorylated STAT1 monomers. The interferon-stimulated gene factor 3 (ISGF-3), a complex formed of phosphorylated STAT1 and STAT2 together with interferon-regulatory factor 9 (IRF9), is exclusively induced in response to type I interferon signalling. Both complexes interact with 
importin- $\alpha 5$ and readily accumulate in the nucleus within minutes after activation (Fagerlund et al., 2002). Once inside the nucleus, the imported complexes facilitate transcription of interferon-responsive genes by association to target sequences in promoter and enhancer regions. STAT1 homodimers bind with high affinity to a palindromic sequence, the so-called interferon-gamma-activated site (GAS). The ISGF-3 complex binds to a different sequence, termed IFN $\alpha$-stimulated response element (ISRE) (Darnell et al., 1994; Horvath, 2000).

STAT inactivation is catalysed by phosphatases located in the cytosol and in the nucleus. In the cytosol, the most prominent tyrosine phosphatases are SH2-containing phosphatase 1 (SHP-1), SHP-2, and protein tyrosine phosphatase 1B (PTP1B). In the case of STAT1 and STAT3, an important mechanism of inactivation is dephosphorylation in the nucleus by the T cell protein tyrosine phosphatase TC-45 (Hoeve et al., 2002; Levy and Darnell, 2002; Rawlings et al., 2004).

The most well-studied inhibitory mechanism to counteract JAK/STAT signalling is by the expression of suppressor of cytokine signalling (SOCS) proteins. These proteins are components in a negative feedback loop since most SOCS protein encoding genes are known targets of STAT-regulated gene transcription (Starr and Hilton, 1999; Levy and Darnell, 2002). Eight members of the SOCS protein family have been characterized and termed SOCS1-SOCS7 and cytokine-inducible SH2-domain containing protein (CIS). Some SOCS proteins execute their action of JAK/STAT signal inhibition by binding to the catalytic subunit of the receptor-bound JAKs, thereby hindering the autophosphorylation of the kinases. CIS has a different mode of action in competing with STAT proteins for the receptor binding site. The best studied of the SOCS protein family are SOCS1 and SOCS3 since the genetic knockout of these factors in mice results in complex and distinct impairments of immune regulation (Alexander, 2002; Rawlings et al., 2004; Linossi and Nicholson, 2015).

Another system of JAK/STAT signal inhibition functions through the expression of PIAS. This class of proteins has been shown to interact with numerous signalling pathways, the JAK/STAT and nuclear factor $\kappa \mathrm{B}(\mathrm{NF} \kappa \mathrm{B})$ pathway being the best studied. They are thought to interact with dimeric STAT complexes by blocking the DNA-binding activity to prevent STATs from binding to promoters and, additionally, by the recruitment of co-repressors to stop the initiation of transcription. PIAS regulation does not affect all STAT-inducible genes to the same extent, indicating a mechanism of differential regulation. While some genes have been shown to be upregulated in PIAS1 knockdown, other genes remain unaffected (Liu et al., 2004; Shuai, 2006). 
To study the function of STAT proteins in more detail, several different genetic knockout models for members of the STAT protein family have been generated. Since STAT1 mediates the intracellular signal transduction of both type I and type II interferons, the complete functional knockout of this transcription factor results in a complete absence of the typical response to IFN $\alpha$, IFN $\beta$ and IFN $\gamma$. These mice show a high susceptibility to a large variety of microbial pathogens while remaining otherwise healthy and indistinguishable from wildtype littermates when kept under pathogen-free conditions (Durbin et al., 1996; Meraz et al., 1996). Using an ex vivo ischemia/reperfusion model of Langendorff perfusion of the heart, it was demonstrated that STAT1-deficient mice displayed reduced infarct size. Cardiomyocytes of the STAT1-knockout mouse line favoured autophagy over apoptosis signalling, resulting in higher cell survival rates in the peri-infarct region (McCormick et al., 2012).

The STAT1-F77A knockin mouse lacks the critical phenylalanine residue located in the Nterminus which is needed to facilitate tetramer stabilization. Owing to the exchange of this amino acid residue to alanine, cooperative DNA binding is rendered impossible. The F77A point mutation leaves type I interferon signal transduction mostly unaffected, but results in severely impaired transcriptional activation upon IFN $\gamma$ signalling. Mice lacking N-domainmediated tetramer stabilization are protected from viral infection and have a lower mortality under treatment with lipopolysaccharide (LPS). However, the transgenic mouse line showed higher rates of mortality when infected with Listeria monocytogenes, indicating impairments in antibacterial but not antiviral response (Begitt et al., 2014).

For some STAT proteins, especially for STAT1, it has been shown that they upregulate their own expression in a self-inducing manner. Upon IFN $\gamma$ stimulation, the intracellular levels of unphosphorylated STAT1 are increased and remain elevated for more than one day. During this time, a set of immunomodulatory genes, among them several genes important in antiviral immune regulation, are overexpressed, as shown in fibroblasts and mammary gland epithelial cells (Cheon and Stark, 2009). A genetic knockin of Stat1 which is constitutively unphosphorylated at this critical tyrosine residue was generated. The resulting mutant displayed a reduced and delayed response to type I and type II interferons. Furthermore, mice expressing tyrosine-phosphorylation-dead STAT1 were more susceptible to infection with Legionella pneumophila but not to Listeria monocytogenes compared with the complete functional knockout of STAT1 (Majoros et al., 2016).

As a component of the ISGF-3 complex, STAT2 is needed to transduce type I interferon signals. Mice lacking STAT2 protein expression are viable, but show a distinct 
immunological phenotype in being more susceptible to a broad range of viral pathogens (Park et al., 2000).

The physiological role of STAT3 cannot be evaluated using a complete functional knockout in mice, since this factor contributes to embryonal development, and STAT3deficiency is lethal between embryonic day 6.5 and 7.5 (Takeda et al., 1997b). Using a conditional knockout in Mx-Cre mice, $90 \%$ knockdown efficiency in the liver and $70 \%$ knockdown efficiency in the bone marrow were achieved. These mice showed a markedly reduced production of liver acute phase proteins in response to lipopolysaccharide (LPS) while overall cytokine signalling - pro- as well as anti-inflammatory - was prolonged (Alonzi et al., 2001). A cardiomyocyte-specific deletion of Stat 3 resulted in an increased susceptibility to cardiac damage, higher rates of apoptosis in response to inflammation, and the development of cardiac fibrosis and dysfunction in old age (Jacoby et al., 2003; HilfikerKleiner et al., 2004).

STAT4 is the main transducer of interleukin-12 (IL-12) signalling - a strong inducer of differentiation towards the Th1 lineage in $\mathrm{CD}^{+} \mathrm{T}$ cells. While developing normally and being fertile, STAT4 knockout mice display increased numbers of Th2 cells accompanied by a blockade of the development of Th1 cells (Kaplan et al., 1996b; Takeda and Akira, 2000; Wurster et al., 2000).

Genetic knockout of Stat5a has led to the identification of its role as a signal transducer in mammopoiesis and lactogenesis. While being indistinguishable from wildtype littermates, homozygous STAT5a-deficient mice did not lactate, indicating that, in the process of mammary gland development, STAT5a has a non-redundant role that STAT5b cannot compensate for despite its high sequence homology (Liu et al., 1997). Functional knockout of STAT5b led to a resistance to growth hormone $(\mathrm{GH})$ pulses resulting in dwarfism, elevated plasma GH, low plasma insulin-like growth factor 1 (IGF1), and obesity (Udy et al., 1997). A redundant role for STAT5a and STAT5b in T cells was identified using a double mutant. Peripheral T cells failed to proliferate in response to IL-2 signalling (Moriggl et al., 1999). Using STAT5a-STAT5b double knockin N-domain mutant mice in which STAT5 proteins form dimers but not tetrameric complexes, Lin and coworkers identified a set of cytokineregulated genes whose expression required STAT5 tetramers (Lin et al., 2012). Moreover, the authors demonstrated that the STAT5-double knockin mice had reduced numbers of cluster of differentiation 4-positive $\left(\mathrm{CD}^{+}\right) / \mathrm{CD} 25^{+} \mathrm{T}$ cells, natural killer $(\mathrm{NK})$ cells, and $\mathrm{CD}^{+} \mathrm{T}$ cells 
resulting in impaired cytokine-mediated proliferation. These findings demonstrated that tetramerization of STAT5 is critical for cytokine responses and normal immune function.

STAT6 is activated by IL-4 and IL-13 signalling, stimuli that induce the differentiation of Th2 cells. A mouse model of a complete functional knockout of STAT6 resulted in severely impaired humoral immune responses caused by altered $\mathrm{T}$-helper cell differentiation (Kaplan et al., 1996a; Shimoda et al., 1996; Takeda et al., 1997a).

IL-10 deficiency in mice is associated with increased expression of IFN $\gamma, \mathrm{C}-\mathrm{X}-\mathrm{C}$ motif chemokine ligand 9 (CXCL9) and CXCL10 upon central nervous system (CNS) infection with murine cytomegalovirus. Surprisingly, infiltration of the brain by natural killer (NK) cells and $\mathrm{CD}^{+} \mathrm{T}$ cells was decreased. Expression of L-selectin/CD62L was elevated on CD8 ${ }^{+}$ $\mathrm{T}$ cells obtained from murine cytomegalovirus-infected animals, and migration along a CXCL10 gradient was impaired (Cheeran et al., 2009).

Knockout of the PIAS1 gene was described as a transgenic model for impaired JAK/STAT inhibition (Liu et al., 2004). Mice deficient in PIAS1 expression displayed a set of STAT1regulated genes that were constitutively upregulated (guanylate binding protein 1 [Gbpl], Cxcl9, Cxcl10), while others remained unaffected (Irf1, nitric oxide synthase 2 [Nos2], Socs1). Genetic knockout of Pias1 was partly embryonically lethal, and homozygous pups were smaller than wildtype littermates, while being protected from bacterial infection and showing a stronger antiviral response.

The phenotypes of the above-mentioned transgenic mouse models with impaired JAK/STAT signalling underscore that this signalling pathway plays a major role in innate and adaptive immunity. In summary, type I interferon signalling is known to activate the ISGF-3 complex which induces the transcription of several genes involved in antiviral immunity. Studies using mutants with defective type I interferon signalling have shown that the formation of ISGF-3 is required for protection against a variety of viral pathogens (Horvath, 2000; Levy and Darnell, 2002; Jouanguy et al., 2007). The type II interferon IFN $\gamma$ was identified to be involved in a variety of immunity- and inflammation-related processes including macrophage and $\mathrm{T}$ cell activation. The list of IFN $\gamma$-target genes includes genes coding for antiviral and microbicidal molecules, such as major histocompatibility complex (MHC) molecules, cytokines, chemokines, and phagocytic receptors (Stark, 2007; Hu and Ivashkiv, 2009). In innate immunity, IFN $\gamma$ not only directly activates macrophages but lowdose stimulation was shown to sensitize macrophages resulting in an even more pronounced 
STAT1-dependent response to type II interferon and to inflammatory signals acting through toll-like receptor 4 (TLR4) (Hu et al., 2002).

Signal transduction via STAT3 often acts as a counterbalance to STAT1 signalling. The activation of STAT3 signalling is induced by a variety of factors, among others IL-6, IL-10, epidermal growth factor EGF, and leukemia-inhibitory factor LIF (Wegenka et al., 1993; Riley et al., 1999; Abroun et al., 2015). STAT3 has been attributed a role in several immunological processes, such as autoimmune disease, cancer, and anti-fungal immunity. Dominant negative STAT3 mutations lead to a disease termed hyper-IgE syndrome, which is accompanied by chronic mucocutaneous candidiasis (Nefedova et al., 2004; Frisullo et al., 2006; Harada et al., 2007; Cypowyj et al., 2012).

Within the context of adaptive immunity, STAT1- and STAT3-dependent signal pathways are both important in the lineage decision of $\mathrm{CD}^{+}{ }^{+} \mathrm{T}$ helper (Th) cells. IFN $\gamma$, IL-12, STAT1 and STAT4 form a positive feed-forward loop to induce the master transcription factor of the Th1 cell fate, T-box transcription factor 21 (TBX21/T-bet) (Afkarian et al., 2002). Differentiation towards the Th17 lineage is induced by a combination of IL-6 and transforming growth factor $\beta$ (TGF $\beta$ ) signalling which leads to the induction of the master regulator of the Th17 program, RAR-related orphan receptor $\gamma \mathrm{t}(\mathrm{ROR} \gamma \mathrm{t})$ in a STAT3dependent manner (Bettelli et al., 2006; Ivanov et al., 2006; Korn et al., 2007; Yang et al., 2007). In contrast, regulatory $T$ cells counteract the pro-inflammatory responses executed by Th1, Th2 and Th17 cells. This $\mathrm{CD}^{+} \mathrm{T}$ cell lineage is induced by a combination of TGF $\beta$, IL2 and IL-10 (Chen et al., 2003a; Zheng et al., 2004; Fontenot et al., 2005). IFN $\gamma$ - or STAT1deficient $\mathrm{CD}^{+} \mathrm{T}$ cells are more prone to develop towards the regulatory $\mathrm{T}$ cell fate (Caretto et al., 2010).

Although STAT1- and STAT3-dependent signal pathways induce different and often opposing reactions, stimulation of cells with IFN $\gamma$ can induce STAT3 phosphorylation, and, vice versa, IL-6 - among other activators of STAT3 signalling - also induces STAT1 activation. This effect is especially pronounced in the absence of either of the two STAT proteins (Stark, 2007). Some activators of STAT signalling display a cell type-specific differential regulation of STAT gene induction, e.g. IFN $\beta$ is a strong inducer of STAT1 phosphorylation in monocytes and $\mathrm{CD}^{+} \mathrm{T}$ cells but a poor inducer in $\mathrm{CD} 4^{+} \mathrm{T}$ cells and B cells. In all four cell types, IFN $\beta$ also induces a pronounced STAT3 and STAT5 activation, suggesting that canonical STAT signalling cascades are not applicable to all cell types (van Boxel-Dezaire et al., 2010). The effects of differential regulation and opposing functions were 
recently shown for other cytokines, including IL-21 and IL-27 (Peters et al., 2015; Wan et al., 2015).

\subsection{JAK/STAT signalling, the actin cytoskeleton and the ERM-proteins}

Numerous studies have reported that interferons are engaged in the regulation of cell shape via the actin cytoskeleton. In intestinal epithelial cells, IFN $\gamma$ reduces the expression of the zona occludens protein $\mathrm{ZO}-1$ and induces rearrangements of the apical actin cytoskeleton which results in increased paracellular permeability of the monolayer (Youakim and Ahdieh, 1999). During embryonic development, transient IFN $\gamma$ production accompanied reorganization and re-gain of polarity of trophectoderm cells which had been treated with a protein kinase C agonist (Marcelo and Lefèvre, 2002). Rearrangements of the cytoskeleton have been of interest within the context of activation of different subsets of leukocytes. IL-2, as well as, type I interferons were shown to increase mobility of MHC-I and Ly49A at the cell membrane of NK cells (Bagawath-Singh et al., 2016).

Recruitment to the site of inflammation strongly depends on well-orchestrated cytoskeletal rearrangements. In experiments using a Boyden chamber, it has been demonstrated that complement $\mathrm{C} 3$ isoform a (C3a) in combination with IFNa and IFN $\gamma$ was more potent in inducing F-actin-mediated migration than C3a alone (Gutzmer et al., 2004). Activation of T cells by antigen-presenting cells (APCs) involves rearrangements of the actin cytoskeleton (Valitutti et al., 1995). Migration in response to a chemotactic stimulus is strongly dependent on the correct distribution of signalling molecules and specific proteins to the correct location of the polarized cell (Niggli, 2014). T cells derived from mice deficient for the actin-bundling protein leukocyte plastin did not form proper immunological synapses with antigenpresenting cells resulting in reduced activation, proliferation and cytokine production and less severe symptoms in experimental autoimmune encephalomyelitis (EAE). This shows that proper lamellipodia formation is necessary for T cell activation (Wang et al., 2010).

Not only activation and recruitment of leukocytes are impaired in this context but also the process of migration within the tissue strongly depends on the formation of cellular protrusions that interact with matrix proteins. IFN $\beta$ and IFN $\gamma$ but not IFN $\alpha$ are known to alter actin and paxillin cytoskeletal arrangement, thereby, inhibiting the contact of cell protrusions to fibronectin in U937 cells which hinders migration (Surin et al., 2000). Immature B cells were shown to exclude themselves from antigen-enriched sites in lymphatic organs in an 
IFN $\gamma / \mathrm{PI} 3 \mathrm{~K} / \mathrm{PKC} \alpha$-dependent mechanism which induced actin-cytoskeleton rearrangements that impaired migration (Flaishon et al., 2001). The cytokines $\mathrm{C}-\mathrm{C}$ motif chemokine ligand 2 (CCL2), CXCL9, CCL5, IL-8 and CXCL10 induce microglial migration towards sites of brain inflammation. This migratory process is accompanied by reorganization of F-actin (Cross and Woodroofe, 1999).

In the context of infection with intracellular bacteria, the actin cytoskeleton is often exploited by the pathogen to facilitate bacterial movement. In fighting infection of the intracellular bacterium L. monocytogenes, IL-6 was shown to impair the recruitment of actin by the bacterium, while IL-6 and IFN $\gamma$ both increased the expression of inducible nitric oxide synthase (iNOS) which helps in the defence against intracellular bacteria via production of reactive nitrogen species (Ouadrhiri et al., 1999). The enzyme iNOS was found to be sequestered near the cell membrane to the actin cortex in macrophages where it acts as a first line of defence against intracellular bacteria (Webb et al., 2001).

One mechanism of signalling mediated by the actin cytoskeleton is through the ERM proteins ezrin, radixin and moesin. This class of proteins forms the link between several transmembrane factors and the actin cytoskeleton and is an evolutionary conserved class of cytoskeletal proteins. They are associated with the cytoskeleton and form the link between actin filaments and the intracellular domain of cell surface membrane-spanning proteins. They are involved in a diverse set of cellular processes such being the assembly of the mitotic spindle and stability of filopodia, lamellipodia, microvilli and adhesion sites. Besides facilitating structural integrity of the cell, they perform scaffolding functions which aid signal transduction in response to extracellular stimuli (Takeuchi et al., 1994; Lamb et al., 1997; Furutani et al., 2007; Fehon et al., 2010; Vilmos et al., 2016).

Structurally, ERM proteins contain a highly conserved FERM domain at the N-terminus, an $\alpha$-helical domain at the centre which is predicted to form coiled coils and an actin-binding domain at the carboxy-terminus. The FERM domain consists of three subunits - a ubiquitinlike fold, a four-helix bundle and a phosphotyrosine-binding-like domain - which assemble in a cloverleaf-like structure and facilitate binding to membrane-associated proteins. The Cterminal and $\alpha$-helical domain can fold over the FERM domain in order to cover the interaction sites (Hamada et al., 2000; Bretscher et al., 2002; Pore and Gupta, 2015).

ERM proteins have been attributed several roles in immune mediation such as B and T cell activation and migration. Their role includes the scaffolding of cell-surface molecules as well 
as intracellular signal transmission (Pore and Gupta, 2015). Ezrin, moesin and radixin bind to filamentous but not to globular actin. In most leukocyte subsets, only ezrin and moesin were identified while radixin was not detected (Pestonjamasp et al., 1995; Ivetic and Ridley, 2004). In $\mathrm{T}$ cell activation, the ERM proteins are known to have a role in sequestering membrane proteins that are not directly associated with T cell receptor (TCR) signalling to the distal pole complex (Chauhan and Moore, 2011). During the process of leukocyte extravasation, an accumulation of intercellular adhesion molecule 1 (ICAM1), vascular cell adhesion molecule 1 (VCAM1), ezrin and moesin regulated by Rho/p160 ROCK signalling was needed at the apical pole of the endothelial cells to allow for leukocyte adhesion and transmigration (Barreiro et al., 2002). During the process of diapedesis, Thr567-phosphorylated ezrin is involved in uropod formation and the sequestration of CD44 to the polar cap at the rear end of transmigrating lymphocytes (Lee et al., 2004). T cells derived from patients with systemic lupus erythematosus exhibit higher adhesion and migration capacity, while overexpressing CD44 and Thr567-phosphorylated ezrin. Transfection of healthy donor $\mathrm{T}$ cells with a constitutively active form of ezrin resulted in increased adhesion and migration ( $\mathrm{Li}$ et al., 2007). However, recent experiments using $\mathrm{CD}^{+} \mathrm{T}$ cells that lacked ezrin and moesin showed that ERM proteins were mostly dispensable for migration and diapedesis but severely impaired the interaction with $\beta 1$-integrin and homing to the lymph nodes (Chen et al., 2013).

In the context of innate immunity, moesin was shown to co-precipitate with toll-like receptor 4, lymphocyte antigen 96 (LY96) and CD14 in macrophages. Blockade of moesin using a neutralizing antibody resulted in reduction of TLR4 signal transduction via myeloid differentiation primary response protein 88 (MyD88), interleukin-1 receptor-associated kinase 1 (IRAK1) and TNF receptor-associated factor 6 (TRAF6) (Zawawi et al., 2010). Treatment of peripheral blood monocyte-derived macrophages with IFN $\gamma$ was associated with increased expression of P-glycoprotein and induced a polar redistribution of P-glycoprotein co-localized with ezrin and actin on cell pseudopodia. These results were shown exclusively for macrophages and could not be confirmed for monocytes and lymphocytes (Puddu et al., 1999).

Although expression of the ERM genes is tissue specific, some redundancy has been shown in their function. Mice with a global genetic knockout of moesin developed normally, were fertile and did not show impairments in processes relying on ERM signalling such as platelet aggregation or the formation of stress fibres (Doi et al., 1999). However, moesin deficiency in mice led to an impairment in the slow rolling of neutrophils, resulting in reduced 
recruitment in a peritonitis model (Matsumoto and Hirata, 2016). Mutations in the moesin gene in humans have been linked to primary immunodeficiency (Lagresle-Peyrou et al., 2016). Genetic knockout of radixin in mice led to the birth of healthy appearing pups. However, in these mice, the secretion of bilirubin was hindered leading to high serum concentrations of conjugated bilirubin and liver injury (Kikuchi et al., 2002). Ezrin deficiency in mice results in severe defects in the development of the gastrointestinal system. Usually, these mice die within the first two weeks post-natal (Tamura et al., 2005). Knockdown of ezrin in trophoblast giant cells identified a non-redundant role of ezrin in an immunological context and was shown to be required for sequestering heat-shock protein 70 (HSP70) and facilitating phagocytosis of bacterial pathogens (Watanabe et al., 2009).

Overexpression and increased activity of ezrin have been implicated in the context of cancer. Increased ezrin protein expression in solid tumours has been associated with poor prognosis and increased formation of metastasis (Elzagheid et al., 2008; Leiphrakpam et al., 2014; Cetin et al., 2015). Using hepatocellular carcinoma cell lines, it was shown that its overexpression was associated with an increased ability to migrate, suggesting a role in the formation of metastases (Zhang et al., 2006). In Epstein-Barr virus-associated nasopharyngeal carcinoma, ezrin overexpression was linked to increased motility and invasiveness (Endo et al., 2009). In non-small cell lung cancer cells, interactions of phosphorylated ezrin with the EGF receptor were shown to boost transcription of oncogenic target genes of EGF signalling (Saygideğer-Kont et al., 2016).

ERM phosphorylation is regulated by small GTPases. Deletion of the small GTPase Rasassociated protein 1 (Rap1) in $\mathrm{T}$ cells resulted in reduced homing to lymph nodes and increased invasion of the intestinal mucosa by an ERM phosphorylation-dependent mechanism (Ishihara et al., 2015). Interactions of the Ras homolog (Rho) family GTPases and the ERM proteins have been implicated in the regulation of several factors involved in migration and cell adhesion such as CD44 and solute carrier family 9 member A1 (SLC9A1) (Okamoto et al., 1999; Denker and Barber, 2002). Besides Ras-related C3 botulinum toxin substrate 1 (Rac1), also RhoA was shown to be involved in endothelia interaction with extracellular matrix proteins in an ERM-dependent manner (Menager et al., 1999). Transient signalling via the TCR results in reduced ERM phosphorylation in a Rac-dependent manner which affects polarity and motility of $\mathrm{T}$ cells. This mechanism stalls $\mathrm{T}$ cells in their current position and might allow for the establishment of a proper immunological synapse (CernudaMorollón et al., 2010). 


\subsection{Objectives of this project}

STAT1 displays a variety of ways of facilitating signalling: the tyrosine-phosphorylated molecule binds to STAT2 and IRF9 to mediate IFNa signalling, while in IFN $\gamma$ signalling homodimers of STAT1 are formed. The un-phosphorylated homodimer has recently been shown to be transcriptionally active and, in addition, STAT1 exerts also non-transcriptional effects. The STAT1-F77A knockin mouse is a useful tool to distinguish signalling mediated by the homo-tetramer from other types of signalling. Up to now, this mouse model has been studied mainly within the context of infectious disease. In this project, the focus is shifted from the context of infectious disease where the immune system is activated in order to fight a pathogen towards non-infectious inflammatory diseases. The mouse models of myocardial infarction and EAE are well-established procedures that will be focused on in this investigation. The aim of this study is to increase our understanding of the role of STAT1 in these immunological processes.

This project will assess the role of the N-terminal domain in the transition between the two STAT1 conformational states. Thereafter, the contribution of interferon-activated STAT1 signalling on the expression of the ERM proteins will be studied. In order to evaluate the recruitment of immune cells to sites of myocardial inflammation in vivo, experimental ligation of the left anterior descending artery will be performed in STAT1-F77A knockin mice and the effect will be compared to their wildtype littermates. In addition, the role of STAT1 expression in EAE will be studied. 


\section{Materials and methods}

\subsection{Materials}

\subsubsection{Chemicals and reagents}

Table 1: List of chemicals and reagents

\begin{tabular}{|c|c|}
\hline Name & Company \\
\hline Agarose & Carl Roth, Karlsruhe, Germany \\
\hline Ampicillin & Sigma-Aldrich, Taufkirchen, Germany \\
\hline APS (ammonium persulphate) & Carl Roth \\
\hline Bromophenol blue & Fisher Bioreagents, Pittsburgh, USA \\
\hline BSA (bovine serum albumin) Fraction V & Carl Roth \\
\hline Complete Mini proteinase inhibitor cocktail & Roche, Rotkreuz, Switzerland \\
\hline Concanavalin A & Sigma-Aldrich \\
\hline DAB (3,3'-diaminobenzidine) & Sigma-Aldrich \\
\hline $\begin{array}{l}\text { Desoxy-ATP (adenosine triphosphate) }\left[{ }^{33} \mathrm{P}\right]- \\
\text { labelled }\end{array}$ & Hartmann Analytic, Braunschweig, Germany \\
\hline DMSO (dimethyl sulphoxide) & Applichem, Darmstadt, Germany \\
\hline dNTP (deoxynucleotide triphosphates) & Carl Roth \\
\hline DTT (dithiothreitol) & Applichem \\
\hline EDTA (ethylenediaminetetraacetic acid) & Sigma-Aldrich \\
\hline $\begin{array}{l}\text { EGTA (ethylene glycol-bis( } \beta \text {-amimoethyl } \\
\text { ether)-N,N,N',N'-tetraacetic acid) }\end{array}$ & Carl Roth \\
\hline Entellan & Merck Millipore, Darmstadt, Germany \\
\hline Eosin $\mathrm{Y}$ & Merck Millipore \\
\hline Ethanol, $99.8 \%$ & Carl Roth \\
\hline Ethidium bromide solution $0.625 \mathrm{~g} / \mathrm{ml}$ & VWR Life Science, Erlangen, Germany \\
\hline FBS (fetal bovine serum) Superior & Biochrom, Berlin, Germany \\
\hline Ficoll & Amersham Bioscience, Freiburg, Germany \\
\hline Fluoromount G & Southern Biotech, Birmingham, USA \\
\hline Formaldehyde solution, $37 \%$ & Carl Roth \\
\hline Glycerol, ROTIPURAN $® \geq 99,5 \%$, p. a. & Carl Roth \\
\hline Glycine & Carl Roth \\
\hline $\mathrm{H}_{2} \mathrm{O}_{2}$ (hydrogen peroxide) solution (30\%) & Carl Roth \\
\hline
\end{tabular}


$\mathrm{H}_{2} \mathrm{SO}_{4}$ (sulphuric acid)

$\left[{ }^{3} \mathrm{H}\right]$-Thymidine (tritiated thymidine) (37

$\mathrm{MBq} / \mathrm{ml})$

HEPES (4-(2-hydroxyethyl)-1-

piperazineethanesulphonic acid), Pufferan

$\geq 99,5 \%$, p. a.

HEPES, cell culture grade

Hoechst 33258

Igepal CA-630

Isopropanol

Kanamycin

$\mathrm{KCl}$ (potassium chloride)

L-glutamine

$\mathrm{LiCl}$ (lithium chloride)

LPS

Lymphocyte separation medium

Mayer's haemalum

MegaTran 1.0

Mercaptoethanol

Methanol

$\mathrm{MgCl}_{2}$ (magnesium chloride)

$\mathrm{Na}_{3} \mathrm{C}_{6} \mathrm{H}_{5} \mathrm{O}_{7}$ (trisodium citrate)

$\mathrm{NaCl}$ (sodium chloride)

$\mathrm{NaCl}, 0.9 \%$

$\mathrm{NaHCO}_{3}$ (sodium bicarbonate)

$\mathrm{NaN}_{3}$ (sodium azide)

Na-Pyruvate (sodium pyruvate)

$\mathrm{Na}_{3} \mathrm{VO}_{4}$ (sodium orthovanadate)

NEAA (non-essential amino acids)

PBS (phosphate buffered saline)

Pefabloc

Penicillin/streptomycin

Pertussis toxin

Phenol/chloroform/isoamylalcohol (25:24:1)
Carl Roth

MP Biomedicals, Eschwege, Germany

Carl Roth

Carl Roth

Sigma-Aldrich

Sigma-Aldrich

Carl Roth

Sigma-Aldrich

Merck Millipore

Thermo Fisher

Carl Roth

Sigma-Aldrich

PAA, Pasching, Austria

Merck Millipore

Origene, Rockville, USA

Sigma-Aldrich

Merck Millipore

Sigma-Aldrich

Carl Roth

Carl Roth

B. Braun, Melsungen, Germany

Carl Roth

Thermo Fisher

Thermo Fisher

Acros Organics, Geel, Belgium

Thermo Fisher

Life Technologies, Carlsbad, USA

Roche

Biochrom

List Biological Laboratories, Campbell, USA

Carl Roth 
Poly-dIdC (poly-deoxyinosinic-

deoxycytidylic acid)

Protein A Agarose/Salmon Sperm DNA

Puromycin

RIPA (radioimmunoprecipitation assay)

buffer, $10 \mathrm{x}$

Rotiphorese Gel 30 (Acrylamid/Bisacrylamid (37.5:1)-solution)

Rotiphorese Gel 40 (Acrylamid/Bisacrylamid (29:1)-solution)

Roti-Plast Paraffin

SDS (sodium dodecyl sulphate)

SDS PAGE (polyacrylamide gel

electrophoresis) running buffer, $10 \mathrm{x}$

Sodium acetate

Sodium deoxycholate

TEMED (tetramethylethylenediamine)

Tris-base

Tris- $\mathrm{HCl}$

Triton X-100

Tween-20

Xylene
Carl Roth

Sigma-Aldrich

Merck, Darmstadt, Germany

Sigma-Aldrich

Merck

Carl Roth

Carl Roth

Carl Roth

Carl Roth

Carl Roth

Applichem

Carl Roth

Carl Roth

Carl Roth

Carl Roth

Carl Roth

Merck Millipore

\subsubsection{Culture media}

Table 2: List of media

\begin{tabular}{ll}
\hline Name & Company \\
\hline DMEM (Dulbecco's modified Eagle's & Biochrom \\
medium) (Glucose $4.5 \mathrm{~g} / \mathrm{l}, 580 \mathrm{mg} / \mathrm{l} \mathrm{L-}$ & \\
Glutamin, $110 \mathrm{mg} / \mathrm{l} \mathrm{Na-pyruvate)}$ & \\
DMEM (Glucose $4.5 \mathrm{~g} / \mathrm{l}$, without L- & Biochrom \\
Glutamin, $110 \mathrm{mg} / \mathrm{l} \mathrm{Na-pyruvate)}$ & \\
LB medium (Luria/Miller) & Carl Roth \\
RPMI (Roswell Park Memorial Institute & Lonza, Basel, Switzerland \\
medium) 1640 &
\end{tabular}




\subsubsection{Drugs}

Table 3: List of drugs

\begin{tabular}{ll}
\hline Name & Company \\
\hline Cepetor (medetomidine, $1 \mathrm{mg} / \mathrm{ml})$ & Cp-pharma, Burgdorf, Germany \\
CFA (complete Freund's adjuvant) & Difco Laboratories, Detroit, USA \\
Fentanyl $(0.05 \mathrm{mg} / \mathrm{ml})$ & Rotexmedica, Trittau, Germany \\
Flumazenil $(0.5 \mathrm{mg} / \mathrm{ml})$ & Inresa Arzneimittel, Freiburg im Breisgau, \\
& Germany \\
Isoflurane & Abbvie, Chicago, USA \\
Ketamine & Medistar, Ascheberg, Germany \\
Midazolam (5 mg/ml) & Rotexmedica \\
Novalminsulfon (metamizol, 500 mg/ml) & Zentiva Pharma, Berlin, Germany \\
Revertor (atipamezole hydrochloride (5 & Cp-pharma \\
mg/ml) & \\
Temgesic (buprenorphine hydrochloride & Reckitt Benckiser, Slough, UK \\
0.324 mg/ml) & \\
Xylariem (xylazine hydrochloride) & Ecuphar, Greifswald, Germany
\end{tabular}

\subsubsection{Kits}

Table 4: List of kits

\begin{tabular}{|c|c|}
\hline Name & Company \\
\hline ABsolute Blue QPCR SYBR Green Mix & Thermo Fisher, Waltham, USA \\
\hline Bio-Rad Protein Assay Kit II & Bio-Rad, Hercules, USA \\
\hline Mouse IFN $\gamma$ Enzyme-linked immunosorbent & Biolegend, San Diego, USA \\
\hline \multicolumn{2}{|l|}{ assay (ELISA) MAX Deluxe } \\
\hline Mouse IL-17 ELISA MAX Deluxe & Biolegend \\
\hline PE anti-mouse/rat/human FOXP3 Flow kit & Biolegend \\
\hline PeqGold total RNA Kit & VWR Life Science \\
\hline Qiagen Plasmid Maxi Kit & Qiagen, Hilden, Germany \\
\hline Thermo Scientific Verso ${ }^{\mathrm{TM}} \mathrm{cDNA}$ Synthesis & Thermo Fisher \\
\hline Kit & \\
\hline
\end{tabular}




\subsubsection{Equipment}

Table 5: List of equipment

\begin{tabular}{|c|c|}
\hline Name & Company \\
\hline Bandelin Sonopuls & Bandelin electronic, Berlin, Germany \\
\hline BioBeam 8000, gamma irradiation device & STS, Braunschweig, Germany \\
\hline Biological safety cabinet class II, Herasafe & Thermo Fisher Scientific, Langenselbold, \\
\hline KS 9 & Germany \\
\hline BioPhotometer plus & Eppendorf, Hamburg, Germany \\
\hline Cell harvester Filter Mate & Perkin Elmer, Waltham, USA \\
\hline Centrifuge $5804 \mathrm{R}$ & Eppendorf \\
\hline Embedding machine EG1160 & Leica, Wetzlar, Germany \\
\hline Filter mate cell harvester & Perkin Elmer \\
\hline FLA-5100 scanner & Fuji, Düsseldorf, Germany \\
\hline Flow cytometer FACSCalibur & BD, Heidelberg, Germany \\
\hline Freezing container & Nalgene, Rochester, USA \\
\hline Incubator B $5061 \mathrm{EC}-\mathrm{CO}_{2}$ & Heraeus, Hanau, Germany \\
\hline Speedmill tissue homogenizer & Analytic Jena, Jena, Germany \\
\hline Luminescence counter MicroBeta TriLux & Perkin Elmer, Waltham, USA \\
\hline Microcentrifuge $5415 \mathrm{R}$ & Eppendorf \\
\hline Microscope, fluorescent, Axiovert 200M & Zeiss Oberkochen, Germany \\
\hline Microscope, inverted, Axiovert $40 \mathrm{CFL}$ & Zeiss \\
\hline Microscope, BX51 & Olympus, Tokyo, Japan \\
\hline Microtome SM 2000 R & Leica \\
\hline Mini Vent type 845 & FMI GmbH, Seeheim-Jungenheim, Germany \\
\hline Multifuge $1 \mathrm{~S}-\mathrm{R}$ & Heraeus \\
\hline Neubauer-modified counting chamber & Paul Marienfeld, Königshofen, Germany \\
\hline Odyssey CLx imaging system & LI-COR, Bad Homburg, Germany \\
\hline Orbital shaker & VWR Life Science \\
\hline $\begin{array}{l}\text { Pipettes, Type Research (P1000, P200, P100, } \\
\text { P10, P2.5) }\end{array}$ & Eppendorf \\
\hline Power supply unit & Biometra, Göttingen, Germany \\
\hline Real-time cycler Mastercycler epgradient S & Eppendorf \\
\hline Rocker Duomax 1030 & Heidolph, Schwabach, Germany \\
\hline Semi-dry blotting cell & Peqlab Biotechnologie, Erlangen, Germany \\
\hline
\end{tabular}


SDS PAGE gel running system

Steel Mesh, pore size $40 \mu \mathrm{m}$

Sonicator UW 2070

Sunrise ELISA Reader

Surgical forceps

Surgical scissors

Thermo shaker TS1

Tissue Dehydration System TP1028

Tuberculin metal/glass syringe

UVsolo TS imaging system

Vevo® 2100 System

Waterbath GFL
Biometra

UMG factory, Göttingen, Germany

Bandelin electronic

Tecan, Männedorf, Switzerland

Fine Science Tools, Heidelberg, Germany

Fine Science Tools

Biometra

Leica

Hartenstein, Würzburg, Germany

Analytic Jena

Visualsonics, Toronto, Canada

Schuett biotec GmBH, Göttingen, Germany

\subsubsection{Disposables}

Table 6: List of disposable materials

\begin{tabular}{ll}
\hline Name & Company \\
\hline Cell culture flasks, T75 & Sarstedt, Nümbrecht, Germany \\
Cell culture plates, 6-well, 24-well, 96-well & Sarstedt \\
Cell Scraper 25 cm & Sarstedt \\
Cryovials & Nunc, Roskilde, Denmark \\
Ethilon 9-0 BV-4 5.0 mm 3/8c EH7448G & Ethicon, Norderstedt, Germany \\
surgical silk & \\
FACS tubes & BD \\
Falcon tubes, 15 ml, 50 ml & Sarstedt \\
Filter mat A & Perkin Elmer, Waltham, USA \\
Glass capillary intraEND 100 $\mu 1$ & Brand, Wertheim, Germany \\
Glass cover slip & Thermo Fisher \\
Glass microscope slide & Thermo Fisher \\
Illustra-MicroSpin-G-25 column & GE Healthcare, Little Chalfont, UK \\
Injection needles & B. Braun, Melsungen, Germany \\
InnuSpeed lysis tubes A & Analytic Jena \\
Insulin Syringe, 29 G & B. Braun, Melsungen, Germany \\
MaxiSorp ELISA plates, 96-well, flat bottom & Nunc \\
MeltiLex A scintillator sheets & Perkin Elmer \\
& \\
\hline
\end{tabular}


Microreaction tube, $0.2 \mathrm{ml}, 1.5 \mathrm{ml}, 2 \mathrm{ml}$

Petri Dishes, $10 \mathrm{~cm}$

Pipette Tips (P1000, P100, P10)

Prolene 6-0 C-1 $13 \mathrm{~mm} \mathrm{3/8c} \mathrm{8889H} \mathrm{surgical}$ silk

PVDF (polyvinylidene fluoride) membrane

Immobilon-P

Round bottom tubes, $14 \mathrm{ml}$

Serological pipettes, $5 \mathrm{ml}, 10 \mathrm{ml}$

Syringe, $20 \mathrm{ml}, 10 \mathrm{ml}$
Eppendorf

Corning, New York, USA

Sarstedt

Ethicon, Norderstedt, Germany

Merck Millipore

BD

Sarstedt, Nümbrecht, Germany

BD, Heidelberg, Germany

\subsubsection{Water}

Most solutions referred to in this study were produced using double-distilled $\mathrm{H}_{2} \mathrm{O}$. This quality was reached using the PURELAB Plus purification system (Elga Labwater, Celle, Germany). Water with a resistivity of $18.2 \mathrm{M} \Omega * \mathrm{~cm}$ was produced.

If DNase-, RNase- and proteinase-free water was specifically recommended for the application, Molecular Biology Grade Water (5 Prime, Hilden, Germany) was used.

\subsubsection{Sterilization of material}

Laboratory equipment and solutions used in cell culture or other highly sensitive settings were sterilized using the 5075 ELV autoclave (Tuttnauer Europe B.V., Breda, the Netherlands). If heat sterilization was not recommended for a solution, filter-sterilization was performed using a Minisart NY 25 syringe filter (Sartorius, Göttingen, Germany).

\subsubsection{Oligonucleotides and primers}

All oligonucleotide primers were purchased from Sigma-Aldrich. The online platforms primer3plus and NCBI blast were used to design primers. For the genotyping of the STAT1 knockout mouse line, sequences were taken from (Meraz et al., 1996)). All oligonucleotides were reconstituted in DNase-, RNase-, proteinase-free water and stored at $-20^{\circ} \mathrm{C}$.

\subsubsection{Hybridised oligonucleotides}

Nucleotides were reconstituted at a concentration of $200 \mu \mathrm{mol} / \mathrm{l}$. Hybridization was achieved through mixing equal parts of complementary oligos, heating to $95^{\circ} \mathrm{C}$ for $5 \mathrm{~min}$ and slow cooling to room temperature. 
Table 7: List of hybridised oligonucleotides

\begin{tabular}{|c|c|c|}
\hline \multicolumn{2}{|l|}{ Name } & \multirow{2}{*}{$\begin{array}{l}\text { Sequence } \\
\text { 5'-TTT TCG TTT CCC CGA AAT TGA CGG ATT TCC CCG }\end{array}$} \\
\hline $2 \times \mathrm{xAS}$ & Sense & \\
\hline & & AAA C-3' \\
\hline & Antisense & 5'-TTT TGT TTC GGG GAA ATC CGT CAA TTT CGG GGA \\
\hline & & AAC G-3' \\
\hline \multirow[t]{4}{*}{ EZR long } & Sense & 5'-TTT TCA AAC AAA AAA ACT TAA TTC CTA GAA TTT \\
\hline & & CAG AGA AAT CCC ATG GAG CTT TTT-3' \\
\hline & Antisense & 5'-TTT AAA AAG CTC CAT GGG ATT TCT CTG AAA TTC \\
\hline & & TAG GAA TTA AGT TTT TTT GTT TG-3' \\
\hline \multirow[t]{2}{*}{ EZR short } & Sense & 5'-TTT TTA ATT CCT AGA ATT TCA GAG AAT C-3' \\
\hline & Antisense & 5'TTT TGA TTT CTC TGA AAT TCT AGG AAT TA-3' \\
\hline \multirow[t]{2}{*}{ EZR M1 } & Sense & 5'-TTT TTA ATA GCT ACT ATT TCA GAG AAT C-3' \\
\hline & Antisense & 5'-TTT TGA TTT CTC TGA AAT AGT AGC TAT TA-3' \\
\hline \multirow[t]{2}{*}{ EZR M2 } & Sense & 5'-TTT TTA ATT CCT AGA ATT AGA GAC TAA TC-3' \\
\hline & Antisense & 5'-TTT TGA TTA GTC TCT AAT TCT AGG AAT TA-3' \\
\hline \multirow[t]{2}{*}{ EZR M3 } & Sense & 5'-TTT TTA ATA GCT ACT ATT AGA GAC TAA TC-3' \\
\hline & Antisense & 5'-TTT TGA TTA GTC TCT AAT AGT AGC TAT TA-3' \\
\hline \multirow[t]{2}{*}{ EZR M4 } & Sense & 5'-TTT TTA ATT CCT AGT ATT ACA GAG AAA TC-3' \\
\hline & Antisense & 5'-TTT TGA TTT TC TGT AAT ACT AGG AAT TA-3' \\
\hline \multirow[t]{2}{*}{ M67 } & Sense & 5'-TTT TCG ACA TTT CCC GTA AAT CTG-3' \\
\hline & Antisense & 5'-TTT TCA GAT TTA CGG GAA ATG TCG-3' \\
\hline \multicolumn{3}{|c|}{ Grade of purification: Desalted } \\
\hline
\end{tabular}

Primers used for genotyping and chromatin immunoprecipitation (ChIP) were reconstituted to a concentration of $100 \mu \mathrm{mol} / \mathrm{l}$. Primers used in quantitative real-time polymerase-chain reaction (qRT-PCR) were reconstituted to the concentration of $50 \mu \mathrm{mol} / \mathrm{l}$.

Table 8: List of oligonucleotide primers for genotyping

\begin{tabular}{lll}
\hline Gene & \multicolumn{2}{c}{ Sequence } \\
\hline Stat1-F77A & Forward & 5'-CGC GAA TTG CTA ATA AAA CAA G-3' \\
& Reverse & 5'-TGC TGC TGA GTC CAA ATA AAG-3' \\
Stat1 $w t$ & Forward & 5'-GGC TGA CCT TGG AAC TTG TG-3' \\
& Reverse & 5'-AAG AGT CAG CAG GGG TCT GA-3' \\
& &
\end{tabular}


neomycin

5'-AGC GCA TCG CCT TCT ATC-3'

Grade of purification: Desalted

Table 9: List of oligonucleotide primers for ChIP

\begin{tabular}{lll}
\hline Gene & \multicolumn{2}{c}{ Sequence } \\
\hline$E Z R$ & Forward & 5'-GAA GCC AGA CGT TGA GAA CC-3' \\
& Reverse & 5'-CCG AAG CTC TGG TTA AGT GG-3' \\
IRF1 & Forward & 5'-CTT CGC CGC TAG CTC TAC AAC AG-3' \\
& Reverse & 5'-GCT CCG GGT GGC CTCGGT TCG-3' \\
\multicolumn{2}{l}{ Grade of purification: Desalted }
\end{tabular}

Table 10: List of oligonucleotide primers for quantitative PCR (qPCR)

\begin{tabular}{lll}
\hline Gene & & Sequence \\
\hline ccl2 & Forward & 5'-CCA GTC ACC TGC TGT TAT AAC-3' \\
cxcl10 & Reverse & 5'-TGG AAT CCT GAA CCC ACT TCT-3' \\
& Forward & 5'-ATT CTG AGC CTA CAG CAG AG-3' \\
ezr & Reverse & 5'-GCT TGC AGG AAT AAT TTC AA-3' \\
& Forward & 5'-CAG AGA GAG AGC TCT CGG AG-3' \\
gapdh & Reverse & 5'-TGG CAG TGT ATT CTG CAA GC-3' \\
& Forward & 5'-GAA GGT GAA GGT CGG AGT C-3' \\
ifit1 & Reverse & 5'-GAA GAT GGT GAT GGG ATT TC-3' \\
& Forward & 5'-CAG AAG CCC AGA CTT ACC TG-3' \\
irf1 & Reverse & 5'ATA GGC AGA GAT CGC ATA CC-3' \\
& Forward & 5'-AGC TA GCT GTG CGA GTG TA-3' \\
stat1 & Reverse & 5'-TAG CTG CTG TGG TCA TCA GG-3' \\
& Forward & 5'-CCG TTT TCA TGA CCT CCT GT-3' \\
Grade of purification: HPLC & &
\end{tabular}

\subsubsection{Enzymes and recombinant proteins}

Table 11: List of enzymes

\begin{tabular}{ll}
\hline Name & Company \\
\hline ExtrAvidin (streptavidin-peroxidase) & Sigma-Aldrich \\
InnuTaq DNA polymerase & Analytic Jena
\end{tabular}


InnuTaq HOT-A DNA polymerase

Klenow fragment

Proteinase K

Taq polymerase

Trypsin

Tsp509I
Analytic Jena

New England Biolabs, Ipswich, USA

Carl Roth

Ares Bioscience GmbH, Cologne, Germany

PAA, Pasching, Austria

Thermo Fisher

Table 12: List of recombinant proteins

\begin{tabular}{ll}
\hline Name & Company \\
\hline IFN- $\alpha$, human, recombinant from $E$. coli & Biomol, Hamburg, Germany \\
IFN- $\alpha$, murine, recombinant from $E$. coli & Abcam, Cambridge, Germany \\
IFN- $\gamma$, human, recombinant from $E$. coli & Biomol \\
IFN- $\gamma$, murine, recombinant from $E$. coli & Biomol \\
Myelin oligodendrocyte glycoprotein peptide & Institute for Medical Immunology Charité \\
amino acids 35-55 $\left(\mathrm{MOG}_{35-55}\right)$ & Berlin, Berlin, Germany
\end{tabular}

\subsubsection{Antibodies}

Table 13: List of primary antibodies

\begin{tabular}{lll}
\hline Name & Company & Cat-No. \\
\hline Acetyl-histone H3 & Merck-Millipore, Darmstadt, Germany & $17-10112$ \\
CCR2 AF700, clone 475301 & R\&D Systems, Wiesbaden, Germany & FAB 5538N \\
CD3 & Bio-Rad, Hercules, USA & MCA 1477 \\
CD4 PE, clone GK1.5 & BD Pharmingen, Erembodegem, & 557308 \\
& Belgium & \\
CD4 PE/Cy5, clone H129.19 & Biolegend, San Diego, USA & 130312 \\
CD4 PE e610, clone RM4-5 & eBioscience, Frankfurt a. M.,Germany & $61-0042-80$ \\
CD8 $\alpha$ PE/Cy7, clone 53-6.7 & Biolegend & 100722 \\
CD11b FITC, clone M1/70 & Biolegend & 101206 \\
CD25 FITC, clone 7D4 & BD Pharmingen & 553070 \\
CD44 PE, clone IM7 & BD Pharmingen & 553134 \\
CD62L FITC, clone MEL-14 & Biolegend & 104405 \\
CD68 & Bio-Rad & MCA 1957T \\
Ezrin & Cell Signaling Technology, Cambridge, & 3145 \\
& UK &
\end{tabular}


Ezrin/radixin/moesin

Glyceraldehyde 3-phosphate

dehydrogenase (GAPDH),

clone $14 \mathrm{C} 10$ rabbit $\mathrm{mAB}$

Ly-6C APC/Cy7, clone

HK1.4

Ly-6G PE, clone RB6-8C5

Myeloperoxidase

NK-1.1 APC/Cy7, clone

PK136

Normal rabbit IgG

Phospho-ERM (ezrin

[Thr567]/radixin

[Thr564]/moesin [Thr558])

p-STAT1, clone 58D6

p-STAT3

STAT1, (C-24)

STAT1 $\alpha$ p91, (M-23)

STAT3, (H-190)

TCR $\beta$ FITC, clone H57-597
Cell Signaling Technology

3142

Cell Signaling Technology

2118

Biolegend, San Diego, USA

128025

BD Pharmingen

553128

Thermo Fisher

PA5-16672

Biolegend

108723

Merck-Millipore

12-370

Cell Signaling Technology

3141

Cell Signaling Technology

9167

Cell Signaling Technology

9131

Santa Cruz, Dallas, USA

sc-345

Santa Cruz

sc-591

Santa Cruz

sc-7179

BD Pharmingen
553171

Table 14: List of secondary antibodies

\begin{tabular}{llc}
\hline Name & Company & Cat-No. \\
\hline Biotinylated anti-rabbit IgG $(\mathrm{H}+\mathrm{L})$ & Vector Laboratories Inc, & BA-1000 \\
derived from goat & Burlingame, USA & \\
Biotinylated anti-rat IgG $(\mathrm{H}+\mathrm{L})$ derived & Vector Laboratories Inc, & BA-4001 \\
from rabbit & Burlingame, USA & \\
Goat anti-rat secondary antibody, Alexa- & Thermo Fisher & A-21247 \\
Fluor 647 conjugated & & \\
Donkey anti-rabbit secondary antibody, & LI-COR Biosciences, Bad & $926-32213$ \\
IRDye 800CW conjugated & Homburg, Germany &
\end{tabular}




\subsubsection{Plasmids and constructs}

Table 15: List of plasmids and constructs

\begin{tabular}{lll}
\hline Name & Description & Reference \\
\hline pSTAT1 & Full-length human STAT1 cloned into the & (Begitt et al., 2000) \\
& pcDNA3.1 mammalian expression vector & \\
& (Thermo Fisher) & \\
pSTAT1-DE92/96RR & pSTAT1 double missense mutation with & (Staab et al., 2015) \\
& exchange of aspartate 92 and glutamate 96 to & \\
pSTAT1-F77A & arginine & \\
& pSTAT1 point mutation with exchange of & (Meyer et al., \\
pSTAT1-GFP & phenylalanine 77 to alanine & 2004) \\
& Recombinant human STAT1 $\alpha$ (aa 1-746) & (Begitt et al., 2000) \\
& protein fused to green fluorescent protein & \\
& (GFP) at the carboxy-terminus cloned into the & \\
& pEGFP-N1 vector (Clontech Laboratories Inc, & \\
& Mountain View, USA) & \\
pSTAT1-GFP encoding the F77A point & (Meyer et al., \\
& mutation as a STAT1 GFP fusion protein & 2004)
\end{tabular}

2.1.13 Cell lines

Table 16: List of cell lines

\begin{tabular}{lll}
\hline Name & Characteristics & Origin \\
\hline HeLa & Human adherent epitheloid cervix carcinoma & (Scherer et al., 1953) \\
& cell line; positive for HPV16 & Kind gift from Professor Uwe \\
HL-60 & Human suspension cell line derived from & Vinkemeier, Nottingham \\
& peripheral blood promyeloblast leukemia & Kind gift from Professor \\
& & Matthias Dobbelstein, Göttingen \\
Jurkat & Human suspension cell line derived from & (Gillis and Watson, 1980) \\
& T lymphocyte leukemia & Kind gift from Professor \\
& & Matthias Dobbelstein, Göttingen \\
U3A & Human adherent epitheloid fibrosarcoma line; $;$ & (McKendry et al., 1991) \\
& deficient in STAT1 expression & Kind gift from Professor Uwe
\end{tabular}




\subsubsection{Animals}

Some of the experiments in this project were performed in mice as a model organism. Mice were kept in individually ventilated cages in the animal facilities of the University Medical Centre, Göttingen. All experiments performed were in line with the current law and approved by the Lower Saxony State Office for Consumer Protection and Food Safety (Niedersächsisches Landesamt für Verbraucherschutz und Lebensmittelsicherheit, LAVES).

Table 17: List of mouse lines

\begin{tabular}{lll}
\hline Name & Characteristics & Origin \\
\hline STAT1-F77A & Mouse line carrying a point mutation & (Begitt et al., 2014) \\
knockin & resulting in a substitution of alanine & \\
& in position 77 for phenylalanine on a & \\
& C57B16/N background (Charles & \\
& Rivers, Sulzfeld, Germany) & \\
& Mouse line with deletion of several & (Meraz et al., 1996) \\
STAT1 knockout & exons of STAT1 resulting in a & Purchased from Taconic \\
& complete functional knockout; & Farms, Cologne, Germany \\
& obtained on an 129/Sv background & \\
& and backcrossed for more than six & \\
& generations onto a C57BL6/N & \\
& (Charles Rivers, Sulzfeld, Germany) & \\
& genetic background &
\end{tabular}

\subsubsection{Software}

Table 18: List of software

\begin{tabular}{lll}
\hline Name & Description & Distributor \\
\hline AnalySIS^B & $\begin{array}{l}\text { Life science imaging } \\
\text { software }\end{array}$ & Olympus \\
CellQuestPro & Operating and analysis & BD \\
& software for cell sorter & \\
CorelDRAW 12 & Vector Graphics editor & Corel Corporation, Ottawa, \\
& & Canada
\end{tabular}


Fiji

IBM SPSS, Version 23

Image J

Magellan6

Microsoft Excel

Realplex ep

SigmaPlot

TINA v 2.0

Vevo® 2100 Software
Add-on to the Image J

software with focus on

biological-image analysis

Statistics software

Java-based image processing program

Operating and analysis

software for ELISA reader

Spreadsheet analysis program

Operating software for

Eppendorf real-time cycler

Statistics software

Operating software for FLA-

5100 scanner

Operating and analysis

software for the Vevo® 2100

imaging system
Public domain; add-on to ImageJ (Schindelin et al., 2012)

IBM, Armonk, USA

Public domain; developed at NIH by Wayne Rasband

(Schneider et al., 2012)

Tecan, Mannedorf,

Switzerland

Microsoft Corporation,

Redmond, USA

Eppendorf

Systat Software GmbH,

Erkrath, Germany

Visualsonics, Toronto,

Canada 


\subsection{Methods}

\subsubsection{Cell culture}

\subsubsection{Unfreezing of established tumour cell lines}

To unfreeze established tumour cell lines (namely U3A, HeLa, Jurkat and HL-60), a cryovial containing $1.5 \times 10^{6}$ cells was taken from the $-80^{\circ} \mathrm{C}$ freezer and quickly thawed in a water bath at $37^{\circ} \mathrm{C}$. As soon as most of the cell suspension was liquid, the content of the tube was transferred to $10 \mathrm{ml}$ pre-warmed culture medium. This diluted suspension was centrifuged for $5 \mathrm{~min}$ at $65 \mathrm{x} \mathrm{g}$ at room temperature. The supernatant was discarded, the pellet was resuspended in $10 \mathrm{ml}$ pre-warmed culture medium and transferred to a T75 culture flask to allow the cells to attach and grow. The following culture media were used: for U3A cells DMEM (with L-glutamine) with $10 \%$ FBS, $1 \%$ penicillin/streptomycin, and $0.008 \%$ puromycin and for HeLa, HL-60, and Jurkat cells RPMI 1640, supplemented with $10 \%$ FBS and $1 \%$ penicillin/streptomycin. Commercially obtained PBS (Gibco) was used for all cellculture applications.

\subsubsection{Freezing of established tumour cell lines}

To freeze cells from an established tumour cell line, the cells were harvested by digestion of cell-cell junctions using trypsin after washing the cells with PBS for adherent cells or direct centrifugation of suspension culture cells. The incubation time with trypsin was 2 min for U3A cells and $4 \mathrm{~min}$ for HeLa cells. The cells were collected and spun down at $65 \mathrm{x} \mathrm{g}$ for $5 \mathrm{~min}$. The supernatant was discarded, the pellet was resuspended in the respective freezing medium and aliquots of $1.5 \times 10^{6}$ cells in $1 \mathrm{ml}$ freezing medium were transferred to cryotubes. The tubes were transferred to a freezing container to allow gradual cooling to $-80^{\circ} \mathrm{C}$ and stored at $-80^{\circ} \mathrm{C}$. Culture medium supplemented with $20 \% \mathrm{FBS}$ and $10 \%$ DMSO was used for freezing HeLa, HL60, Jurkat, and U3A cells.

\subsubsection{Maintenance, passaging and seeding of established tumour cell lines}

The established tumour cell lines were cultured in a humidified atmosphere at $37^{\circ} \mathrm{C}$ and $5 \%$ $\mathrm{CO}_{2}$ (HeLa, HL-60, Jurkat, and U3A cells). The cells were passaged every 2 to 3 days. For this, the suspension cells were pelleted by centrifugation at $65 \mathrm{x}$ g for $5 \mathrm{~min}$. The supernatant was discarded, and the pellet resuspended in culture medium. An aliquot was inoculated with fresh culture media in a new T75 flask (typical passage as 1:8 or 1:10 dilution). Adherent cells were washed with PBS and trypsinized. Trypsin activity was stopped by addition of twice the 
amount of complete culture medium. An aliquot from this suspension was used to start a new passage in a T75 flask (typical passage as 1:8 or 1:10 dilution). For the plating, cells were counted before being seeded. For protein and RNA analysis, 250,000 cells were seeded in each well of a 6-well culture plate. This corresponds to a density of approximately $1.56 \times 10^{5}$ cells per $\mathrm{cm}^{2}$.

\subsubsection{Routine check for mycoplasma infection in established tumour cell lines}

To check for infection with mycoplasma, cells were stained with Hoechst dye. Suspension cells were spun down at $100 \mathrm{xg}$ and resuspended in methanol for a 15 min fixation. After having been spun down again, the cells were resuspended in Hoechst dye diluted 1:5,000 in methanol and incubated at $37^{\circ} \mathrm{C}$ for $15 \mathrm{~min}$. Cells were spun down yet again, washed with PBS and resuspended in PBS. For the analysis, $50 \mu 1$ of the cell suspension were placed on a glass microscope slide and covered with a cover slip. Adherent cells were fixed with $4 \%$ formaldehyde in PBS for $15 \mathrm{~min}$ at room temperature. Cells were washed twice with PBS and once with $\mathrm{H}_{2} \mathrm{O}$ and stained with Hoechst dye diluted 1:5,000 in PBS. Again, cells were washed twice with PBS, once with $\mathrm{H}_{2} \mathrm{O}$, and mounted using Fluoromount $\mathrm{G}$ mounting medium. Cells were routinely analysed for Hoechst staining in the cytoplasm to detect mycoplasma infection using the Axiovert 200M fluorescent microscope at $340 \mathrm{~nm}$ and a magnification of $40 x$.

\subsubsection{Treatment of cultured cells with different stimuli}

Prior to protein or RNA extraction, cultured cells were treated with different stimuli. To this end, the respective stimulus (e.g. IFN $\gamma$ ) was diluted in pre-warmed culture medium at the desired concentration for adherent cells or at five times the concentrations used for suspension cells. For adherent cells, the culture medium was replaced by the medium containing the stimulus. In the case of suspension cells, $25 \%$ of the culture volume was added from the cytokine-containing medium. For the RNA extraction, culture medium was replaced with culture medium containing only $0.5 \%$ FBS $16 \mathrm{~h}$ prior to the beginning of the stimulation, and stimulation with cytokines was also performed in low serum-culture medium. If not stated differently, treatment concentrations were as follows: human IFN $\gamma ; 50 \mathrm{ng} / \mathrm{ml}$, human IFN $\alpha$; $50 \mathrm{ng} / \mathrm{ml}$, murine IFN $\gamma ; 5 \mathrm{ng} / \mathrm{ml}$, and murine IFN $\alpha ; 5 \mathrm{ng} / \mathrm{ml}$. Treatment with vanadate was carried out using $0.8 \mathrm{mmol} / \mathrm{l}$ sodium vanadate and $0.2 \mathrm{mmol} / 1 \mathrm{H}_{2} \mathrm{O}_{2}$. 


\subsubsection{Transfection of cultured cells with plasmid DNA}

Cultured eukaryotic cells were transfected with different vectors using MegaTran 1.0 according to the manufacturer's recommendation. For the 6-well plate scale, $160 \mu \mathrm{l}$ of $150 \mathrm{mM} \mathrm{NaCl}$ solution were mixed with $1.6 \mu \mathrm{g}$ of DNA and $4.8 \mu \mathrm{l}$ of MegaTran 1.0 per well using a vortex and incubated at room temperature for $10 \mathrm{~min}$. For each well, $160 \mu 1$ of this solution was added to eukaryotic cells that had grown to 50-70\% confluence and blended with the culture supernatant by gentle agitation. Cells were cultured for a further $24 \mathrm{~h}$ before stimulation to allow for uptake and expression of the vector.

\subsubsection{Protein biochemistry}

\subsubsection{Fractionated protein extraction}

For protein extraction, cells grown in 6-well-culture plates or a single cell suspension were used. For suspension cells, the cells were collected and harvested by centrifugation for 4 min at $1,500 \mathrm{x}$. They were washed with PBS and spun down again. The pellet was resuspended in $50 \mu \mathrm{l}$ complete cytosolic protein lysis buffer $(20 \mathrm{mM}$ HEPES, $10 \mathrm{mM} \mathrm{KCl}, 1 \mathrm{mM}$ EDTA, $100 \mu \mathrm{M} \mathrm{Na} \mathrm{VO}_{4}, 10 \%$ glycerol, $0.1 \%$ IGEPAL CA-630, pH 7.4, supplemented with $1 \%$ Complete proteinase inhibitor cocktail (reconstituted), $3 \mathrm{mM}$ DTT, $0.4 \mathrm{mM}$ Pefabloc) and incubated on ice for $5 \mathrm{~min}$. In order to harvest adherent cells, they were washed twice with PBS, and $50 \mu \mathrm{l}$ of complete cytosolic protein lysis buffer were added to the culture dish. The cells were incubated with the lysis buffer for $5 \mathrm{~min}$, harvested using a cell scraper and then transferred to a $1.5 \mathrm{ml}$ microreaction tube. The cells in complete cytosolic lysis buffer were spun down for $15 \mathrm{sec}$ at $4^{\circ} \mathrm{C}$ and $16,100 \mathrm{x} \mathrm{g}$. The supernatant was transferred to a fresh microreaction tube and centrifuged again for $5 \mathrm{~min}$ at $4^{\circ} \mathrm{C}$ and $16,100 \mathrm{x} \mathrm{g}$. The resulting cleared supernatant was collected as cytosolic protein extract. The pellet from the initial centrifugation step was resuspended in $50 \mu$ l complete nucleic protein lysis buffer $(20 \mathrm{mM}$ HEPES, $420 \mathrm{mM} \mathrm{KCl,} 1 \mathrm{mM}$ EDTA, $100 \mu \mathrm{M} \mathrm{Na}_{3} \mathrm{VO}_{4}, 20 \%$ glycerol, pH 7.4, supplemented with $1 \%$ Complete proteinase inhibitor cocktail, $3 \mathrm{mM}$ DTT, and $0.4 \mathrm{mM}$ Pefabloc) and incubated on ice for $30 \mathrm{~min}$. The lysate was centrifuged for $15 \mathrm{~min}$ at $4^{\circ} \mathrm{C}$ and $16,100 \mathrm{xg}$. The supernatant was collected as nucleic extract. Finally, the cytosolic and nucleic extracts were pooled. If the protein extracts were to be used in electrophoretic mobility shift assay (EMSA) analysis, they were stored without any further processing at $-80^{\circ} \mathrm{C}$. If the protein extracts were to be used in Western blotting analysis, $14.4 \mu \mathrm{l}$ of $6 \mathrm{x}$ Laemmli buffer (350 mM Tris- $\mathrm{HCl}, 8 \%$ SDS, $30 \%$ glycerol, $10 \%$ mercaptoethanol, $0.04 \%$ bromophenol, $\mathrm{pH} 7.4$ ) were added to the $80 \mu \mathrm{l}$ pooled protein sample. The extracts were boiled at $95^{\circ} \mathrm{C}$ for $3 \mathrm{~min}$ and stored at $-20^{\circ} \mathrm{C}$. 


\subsubsection{Direct protein extraction from bone marrow cells}

Proteins from treated murine bone marrow were obtained using a direct approach: Following isolation of the bone marrow and production of a single cell suspension, cells were collected by centrifugation for $4 \mathrm{~min}$ at $100 \mathrm{xg}$ at $4^{\circ} \mathrm{C}$. The cells were washed once with ice-cold PBS and collected using a second centrifugation step. The pellet was resuspended in $100 \mu 12 \mathrm{x}$ Laemmli buffer (produced by diluting 6x Laemmli buffer in double-distilled $\mathrm{H}_{2} \mathrm{O}$ ) using a $29 \mathrm{G}$ insulin syringe. The extracts were boiled at $95^{\circ} \mathrm{C}$ for $3 \mathrm{~min}$ and stored at $-20^{\circ} \mathrm{C}$ until use. Before being loaded, these samples were diluted 1:1 in SDS-running buffer and again boiled for $3 \mathrm{~min}$ at $95^{\circ} \mathrm{C}$.

\subsubsection{Protein extraction from murine heart}

In order to extract protein from murine heart, cardiac tissue was snap-frozen in liquid $\mathrm{N}_{2}$ after removal of the heart from the mouse and separation of the lower third of the left ventricle (main infarcted area) from the rest of the organ. Protein extraction was achieved by splitting the acquired pieces of tissue in a mortar with liquid $\mathrm{N}_{2}$. Roughly $40 \mathrm{mg}$ of tissue were used for protein extraction. The tissue was placed in $800 \mu$ l complete RIPA buffer supplemented with $1 \%$ complete protease inhibitor cocktail, $1 \%$ PMSF (100 $\mu \mathrm{M}$ in isopropanol), and 1 $\mathrm{mM} \mathrm{Na}_{3} \mathrm{VO}_{4}$ and homogenized using the Speedmill tissue homogenizer with innuSpeed lysis tubes A for $4 \mathrm{x} 45 \mathrm{sec}$. The homogenized sample was incubated on ice for $30 \mathrm{~min}$ and transferred to a fresh reaction tube before sonication at $50 \%$ amplitude, $70 \%$ time for $3 \times 30$ sec. After sonication, debris was spun down at $12,000 \times \mathrm{g}$ for $20 \mathrm{~min}$ at $4^{\circ} \mathrm{C}$. Supernatants were collected as protein extracts and stored at $-80^{\circ} \mathrm{C}$ until use. When used for Western blotting experiments, $40 \mu \mathrm{g}$ of protein were diluted in PBS $(150 \mathrm{mM} \mathrm{NaCl}, 100 \mathrm{mM}$ $\mathrm{Na}_{2} \mathrm{HPO}_{4}, 15 \mathrm{mM} \mathrm{K} \mathrm{HPO}_{4}, 30 \mathrm{mM} \mathrm{KCl}, \mathrm{pH}$ 7.4) and 6x Laemmli buffer was added. These samples were boiled for $5 \mathrm{~min}$ at $95^{\circ} \mathrm{C}$ before being loaded.

\subsubsection{Protein quantification using Bradford assay}

To quantify protein concentrations in samples obtained from whole tissue, the Bradford method was used (Bradford, 1976). Briefly, protein samples were diluted in PBS to reach a total volume of $800 \mu \mathrm{l}$. Two hundred $\mu \mathrm{l}$ of the Bio-Rad protein assay dye reagent was added and incubated with the protein solution for $5 \mathrm{~min}$. The solution was transferred to a semimicro cuvette for spectrometric analysis at $595 \mathrm{~nm}$ using the BioPhotometer plus. The photometer was calibrated for concentrations ranging from $1-25 \mu \mathrm{g} / \mathrm{ml}$ using the BSA standard provided with the Bio-Rad Protein Assay Kit II. 


\subsubsection{Western blotting analysis}

For Western blotting analysis, protein samples containing Laemmli buffer were loaded onto a two-phase $10 \%$ SDS-PAGE gel. Polymerisation of the stacking gel was achieved by adding $25 \%$ stacking gel buffer (500 mM Tris-HCl, $0.4 \%$ SDS, pH 6.8), $16 \%$ Rotiphorese Gel 30, $0.6 \%$ APS, and $0.2 \%$ TEMED in double-distilled $\mathrm{H}_{2} \mathrm{O}$. The separating gel was polymerised by mixing $25 \%$ separating gel buffer (1.5 mM Tris-HCl, $0.2 \%$ SDS, pH 8.8), $33.1 \%$ Rotiphorese Gel 30, $0.03 \%$ APS, and $0.16 \%$ TEMED (in double-distilled $\mathrm{H}_{2} \mathrm{O}$ ). The samples were run at $11 \mathrm{~mA}$ using 1x SDS PAGE running buffer (25 mM Tris-Base, $192 \mathrm{mM}$ glycine, 0.1\% SDS, $\mathrm{pH}$ 8.6) and transferred onto a PVDF membrane using a semidry blotting technique at $80 \mathrm{~mA}$ for $90 \mathrm{~min}$ with transfer buffer $(25 \mathrm{mM}$ Tris-base, $150 \mathrm{mM}$ glycine, $10 \%$ methanol, $\mathrm{pH}$ 8.0). The resulting blot was blocked using $4 \%$ BSA in Tris-buffered saline (TBS) (137 mM NaCl, $10 \mathrm{mM}$ Tris-HCl, pH 7.4) supplemented with $0.1 \%$ Tween-20 (TBST) and incubated overnight at $4{ }^{\circ} \mathrm{C}$ with the primary antibody diluted in $4 \% \mathrm{BSA}$ in TBST. Then, the blot was washed five times for 5 min with TBST before being incubated with the diluted secondary antibody for $1 \mathrm{~h}$ in the dark at room temperature. It was washed 5 times with TBST, once with TBS and scanned using the LI-COR Odyssey CLx imaging system. For reblotting, the membrane was washed three times for $5 \mathrm{~min}$ and three times for $15 \mathrm{~min}$ in TBST. Thereafter, it was incubated overnight with a new primary antibody and developed the next day, as described above. If the first and second antigen to be detected were similar in molecular size, the blot was stripped from its first set of primary and secondary antibodies before analysis. To this end, the blot was incubated with stripping buffer $(62.5 \mathrm{mM}$ Tris- $\mathrm{HCl}$, $2 \% \mathrm{SDS}, 0.7 \%$ mercaptoethanol, $\mathrm{pH} 6.8$ ) at $60^{\circ} \mathrm{C}$ for $60 \mathrm{~min}$. Subsequently, it was thoroughly washed with tap water, then four times for 15 min in TBST, blocked again with $4 \%$ BSA in TBST and incubated overnight with a different primary antibody.

The dilutions of primary antibodies were as follows: anti-ERM 1:1,000; anti-ezrin 1:2,000; anti-GAPDH 1:5,000; anti-phospho-ERM 1:1,000; anti-phosphotyrosine-STAT1 1:1,000; anti-phosphotyrosine-STAT3 1:1,000; anti-STAT1 1:1,000 and anti-STAT3 1:750. Dilution of the IRDye $800 \mathrm{CW}$-conjugated donkey-anti-rabbit secondary antibody was 1:10,000.

\subsubsection{Electrophoretic mobility shift assay}

DNA binding of STAT1 was assessed using electrophoretic mobility shift assay (EMSA). For this technique, purified samples of native protein were incubated with radioactively labelled DNA probes in the presence of a non-specific competitor (poly-dIdC), and the reactions were run on a native polyacrylamide gel. The DNA probe $(0.2 \mu \mathrm{l})$ was mixed with $1 \mu \mathrm{l}$ poly-dIdC 
( $2 \mathrm{mg} / \mathrm{ml}), 1.3 \mu \mathrm{l}$ DTT $(100 \mathrm{mM}), 3.5 \mu 1 \mathrm{H}_{2} \mathrm{O}$ and $2.5 \mu \mathrm{l}$ 5x EMSA loading buffer (100 mM HEPES, $200 \mathrm{mM} \mathrm{KCl}, 5 \mathrm{mM} \mathrm{MgCl} 2,2.5 \mathrm{mM}$ EDTA, $0.5 \mathrm{mM}$ EGTA, $20 \%$ Ficoll) and incubated with $4.5 \mu \mathrm{l}$ or adjusted amounts of protein extracts for the times indicated. The STAT1 C-24 antibody was used in a supershift reaction, while the STAT3 H-190 antibody was used as a negative control. This mixture was loaded onto an equilibrated non-denaturing $4.8 \%$ TBE-polyacrylamide gel (12\% Rotiphorese Gel 40, $2.4 \%$ TBE (89 mM Tris-base, $89 \mathrm{mM}$ boric acid, $2 \mathrm{mM}$ EDTA), $2 \%$ APS, $0.1 \%$ TEMED) and run at $400 \mathrm{~V}$ using $0.25 \mathrm{x}$ TBE. The gel was then pressed against Whatman blotting paper and vacuum-dried. The radioactive signals were detected using transfer of the signal to a phospho-imager film and read by the FLA-5100 scanner. For this reading, the software TINA v 2.0 was used.

\subsubsection{Radioactive labelling of EMSA probes}

Oligonucleotides intended for use in EMSA were annealed by mixing them at a concentration of $100 \mathrm{mmol} / 1$ in DNase-, RNase- and proteinase-free $\mathrm{H}_{2} \mathrm{O}$ and heating the mixture to $95^{\circ} \mathrm{C}$ in a water bath. This was left to slowly cool to room temperature overnight to allow proper annealing and was then stored at $-20^{\circ} \mathrm{C}$ until further use. Radioactive labelling was performed by an end-filling reaction using the Klenow fragment. Five units of the Klenow fragment derived from DNA polymerase I from E. coli were mixed with $5 \mu 1$ 10x Eco-Pol buffer, $0.1 \mathrm{ng}$ annealed oligonucleotides and $8 \mu \mathrm{l}\left[{ }^{33} \mathrm{P}\right]$-labelled ATP and incubated for $25 \mathrm{~min}$ at room temperature. Finally, an excess of non-radioactive dNTPs (6.5 mM of each dNTP) was added for a further $5 \mathrm{~min}$, and the reaction was stopped by addition of $1 \mu \mathrm{l}$ of $0.5 \mathrm{mM}$ EDTA solution. Free nucleotides were eliminated by centrifugation at $700 \mathrm{x} g$ for $3 \mathrm{~min}$ using an Illustra-MicroSpin-G-25 column.

\subsubsection{ELISA}

In order to quantify IL-17 and IFN $\gamma$ production, culture supernatants of cells isolated from lymph node and spleen were collected after $72 \mathrm{~h}$ incubation in restimulation medium (ReMed) (5\% FBS, $0.0002 \%$ mercaptoethanol in $\mathrm{R}_{0}$ medium supplemented with $1 \%$ NEAA, $1 \% \mathrm{Na}$ pyruvate, $1 \%$ L-glutamine, and $1 \%$ penicillin/streptomycin) and analysed using ELISA technique. Cells isolated as described above were seeded in U-bottom 96-well plates at a density of $3 \times 10^{5}$ cells per well for the analysis of lymph node isolates and $6 \times 10^{5}$ cells per well for spleen cells, respectively. $\mathrm{MOG}_{35-55}$ at a final concentration of $20 \mu \mathrm{g} / \mathrm{ml}$ was used as a specific stimulus, while concanavalin A at a concentration of $1.25 \mu \mathrm{g} / \mathrm{ml}$ was used as control. Cells were incubated with the reagents at $37^{\circ} \mathrm{C}$ and $5 \% \mathrm{CO}_{2}$. Each condition was analysed in 
triplicate individually for every animal. After $72 \mathrm{~h}$, supernatants were collected and stored at $-80^{\circ} \mathrm{C}$ until analysis. Measurements were performed using the Mouse ELISA MAX Deluxe kits for IL-17A and IFN $\gamma$ following the manufacturer's recommendations. Briefly, MaxiSorp ELISA plates were coated with $100 \mu \mathrm{l}$ of the capture antibody provided, diluted 1:200 in coating buffer for $16 \mathrm{~h}$ at $4^{\circ} \mathrm{C}$. Unspecific binding sites of the capture antibodies were blocked with Assay Diluent A for $1 \mathrm{~h}$ at room temperature. One hundred $\mu \mathrm{l}$ of diluted supernatants were added to the wells of the plate and incubated for $2 \mathrm{~h}$ at room temperature under mild agitation, each sample was applied in triplicate. Samples were diluted in Assay Diluent A in order to give values within the dynamic range of the assay. Standard curves were generated by inclusion of diluted IL-17A or IFN $\gamma$ at concentrations of $15.6 \mathrm{pg} / \mathrm{ml}, 31.25 \mathrm{pg} / \mathrm{ml}, 62.5$ $\mathrm{pg} / \mathrm{ml}, 125 \mathrm{pg} / \mathrm{ml}, 250 \mathrm{pg} / \mathrm{ml}, 500 \mathrm{pg} / \mathrm{ml}$ and 1,000 pg/ml. Background values were acquired by measurement of pure Assay Diluent A. $100 \mu 1$ of diluted detection antibody was added to each well and incubated for $1 \mathrm{~h}$. Thereafter, the wells were incubated with the avidinconjugated horseradish peroxidase for $30 \mathrm{~min}$. Finally, $100 \mu \mathrm{l}$ of freshly prepared TMB substrate solution was added and incubated with the plate in the dark for $20 \mathrm{~min}$. The chromatic reaction was terminated by adding $100 \mu \mathrm{l} 2 \mathrm{~N} \mathrm{H}_{2} \mathrm{SO}_{4}$ to each well. A thorough rinse using PBS-Tween was performed between each of the steps. Absorbance at $450 \mathrm{~nm}$ and $570 \mathrm{~nm}$ was determined using the Sunrise ELISA plate reader. Difference values used for further analysis were calculated as the absorbance at $570 \mathrm{~nm}$ subtracted from the absorbance at $450 \mathrm{~nm}$. Quantitative assessment of the data was performed using the standard curve.

\subsubsection{DNA analysis}

\subsubsection{DNA extraction from tail biopsies}

To extract DNA from mouse tissue samples (e.g. tail biopsy), the tissue sample was lysed in $500 \mu \mathrm{l}$ tail extraction buffer (100 mM Tris, 5 mM EDTA, $200 \mathrm{mM} \mathrm{NaCl}, 0.2 \%$ SDS, pH 8.5) and $10 \mu \mathrm{l}$ proteinase $\mathrm{K}(20 \mathrm{mg} / \mathrm{ml})$ overnight at $56^{\circ} \mathrm{C}$ and $250 \mathrm{rpm}$ in a thermo-shaker. Proteinase $\mathrm{K}$ was inactivated by a $5 \mathrm{~min}$ incubation at $90^{\circ} \mathrm{C}$. The lysate was centrifuged at $16,100 \mathrm{x} \mathrm{g}$ and $10^{\circ} \mathrm{C}$ for $15 \mathrm{~min}, 350 \mu \mathrm{l}$ of the supernatant was collected, and the DNA was precipitated by adding $500 \mu 1$ isopropanol. The sample was mixed thoroughly by vortexing and centrifuged for $5 \mathrm{~min}$ at 16,100 $\mathrm{xg}$. The supernatant was discarded, the pellet was washed twice with $70 \%$ ethanol, air-dried and finally resuspended in $50 \mu 1 \mathrm{TE}$ buffer (10 mM Trisbase, $1 \mathrm{mM}$ EDTA, $\mathrm{pH} 7.5$ ). The DNA was heated to $48^{\circ} \mathrm{C}$ for $30 \mathrm{~min}$ and stored at $4^{\circ} \mathrm{C}$ overnight before analysis to allow complete resuspension. 


\subsubsection{Genotyping of STAT1-F77A knockin mice}

Genotyping of STAT1-F77A knockin mice was achieved by PCR amplification and subsequent analysis of a restriction-fragment size polymorphism. The respective fragment was amplified using the primer pair stat $1 \mathrm{~F} 77 \mathrm{~A}$ at a final concentration of $0.4 \mu \mathrm{mol} / \mathrm{l}, 2.5 \mu \mathrm{l}$ of $10 \mathrm{x}$ reaction buffer, $0.5 \mu \mathrm{l}$ of $50 \mathrm{mM} \mathrm{MgCl}_{2}$ solution, $1 \mu \mathrm{l}$ of $\mathrm{dNTP}$ mix $(10 \mu \mathrm{mol} / \mathrm{l})$, $17.25 \mu \mathrm{l}$ of DNase-, RNase- and proteinase-free water and $0.25 \mu \mathrm{l}$ of Taq polymerase for each sample. $1.5 \mu \mathrm{l}$ of purified DNA solution was added. The reaction was transferred to a thermocycler and run with the following program: $95^{\circ} \mathrm{C}$ for $3 \mathrm{~min}$, followed by 41 cycles of denaturation at $95^{\circ} \mathrm{C}$ for $15 \mathrm{~s}$, annealing at $61^{\circ} \mathrm{C}$ for $15 \mathrm{~s}$, and extension at $72^{\circ} \mathrm{C}$ for $15 \mathrm{sec}$ with a final elongation at $72^{\circ} \mathrm{C}$ for $7 \mathrm{~min}$. The resulting PCR product was run on a $1 \%$ agarose gel at $85 \mathrm{~mA}$ for $45 \mathrm{~min}$ and analysed using the UVsolo TS imaging system to check for proper product amplification. A total of $5 \mu 1$ of the product were incubated with $0.3 \mu 1$ of Tsp509I, $1.5 \mu \mathrm{l}$ of reaction buffer B and 7.2 $\mu 1$ of DNase, RNase- and proteinase-free water overnight at $65^{\circ} \mathrm{C}$. The digested PCR product was tested using a $3 \%$ agarose gel run at $85 \mathrm{~mA}$ for $60 \mathrm{~min}$. Since the F77A mutation eliminates a Tsp509I-restriction site, fragmentlength polymorphism was exploited for genotype analysis. Due to the loss of a Tsp509I site caused by mutation F77A, the STAT1F77A-derived PCR product showed an additional $234 \mathrm{bp}$ fragment, which was cleaved into two fragments of similar size in the case of wildtype-derived DNA.

\subsubsection{Genotyping of STAT1-KO mice}

Genotyping of STAT1-KO mice was achieved by a PCR setup resulting in the amplification of a native or neomycin cassette-related fragment of the $\mathrm{KO}$ allele or both with a common upstream primer and a reverse primer located either in the deleted sequence or in the neomycin-resistance gene. For this purpose, the DNA was prepared and purified as described above. The PCR reaction included $2.5 \mu \mathrm{l}$ of $10 \mathrm{x}$ buffer containing $\mathrm{MgCl}_{2}, 1 \mu \mathrm{l}$ each of stat1 wildtype forward and reverse and neomycin primer, $0.625 \mu 1$ of dNTP, $0.15 \mu 1$ of innuTaq Hot A polymerase, $1 \mu \mathrm{l}$ of DNA and $17.7 \mu \mathrm{l}$ of DNase- RNase- and proteinase-free water. The PCR conditions were $95^{\circ} \mathrm{C}$ for $4 \mathrm{~min}$, followed by 40 cycles of denaturation at $95^{\circ} \mathrm{C}$ for $45 \mathrm{~s}$, annealing at $60^{\circ} \mathrm{C}$ for $45 \mathrm{~s}$, and extension at $72^{\circ} \mathrm{C}$ for $210 \mathrm{sec}$ with a final elongation at $72^{\circ} \mathrm{C}$ for $10 \mathrm{~min}$. The resulting PCR product was run on a $1 \%$ agarose gel at $85 \mathrm{~mA}$ for $45 \mathrm{~min}$ and visualized using the UVsolo TS imaging system. The PCR product from the native gene was $1.7 \mathrm{~kb}$, while the corresponding product length of the transgene was $1.3 \mathrm{~kb}$. 


\subsubsection{Plasmid amplification}

For plasmid amplification, chemically competent bacteria of the E. coli strain DH5 $\alpha$ were transformed using the heat-shock method (Cohen et al., 1972). Briefly, $100 \mu 1$ of DH5 $\alpha$ cells were slowly unfrozen and incubated on ice with $1 \mu \mathrm{g}$ of the desired plasmid DNA. The bacteria were then exposed to a $30 \mathrm{sec}$ heat shock at $42^{\circ} \mathrm{C}$ with a subsequent 2 min-incubation on ice. The reaction was transferred to $1 \mathrm{ml}$ of antibiotic-free LB medium and incubated for $1 \mathrm{~h}$ at $37^{\circ} \mathrm{C}$ with $225 \mathrm{rpm}$ of orbital agitation before being spread on a pre-warmed agarose plate containing either ampicillin (for the plasmids derived from pcDNA3.1) or kanamycin (for the plasmids derived from pEGFP-N1). After incubation lasting $16 \mathrm{~h}$, a colony was picked and inoculated with $2 \mathrm{ml}$ of LB medium containing the respective antibiotic and grown for $8 \mathrm{~h}$, before $1 \mathrm{ml}$ of this pre-culture was transferred to $100 \mathrm{ml}$ of LB medium with antibiotic and incubated overnight. The bacteria grown in this culture were centrifuged at $6,000 \times \mathrm{g}$ for $15 \mathrm{~min}$. For the isolation of plasmid DNA, the Qiagen Plasmid Maxi kit was used according to the manufacturers' instructions. The DNA was resuspended in DNAse-, RNAse- and proteinase-free water. The concentration was adjusted to $1 \mu \mathrm{g} / \mu \mathrm{l}$ after determination by UV spectrophotometry. Plasmid DNA was stored at $-20^{\circ} \mathrm{C}$.

\subsubsection{ChIP analysis}

For chromatin immunoprecipitation (ChIP), Jurkat cells were grown in $10 \mathrm{ml}$ suspension culture until they reached $80 \%$ confluence and treated with $50 \mathrm{ng} / \mathrm{ml} \mathrm{IFN} \gamma$ for $45 \mathrm{~min}$ or left untreated. Cells were harvested, washed twice with PBS and resuspended in $1.5 \%$ formaldehyde in PBS. Cells were fixed for $15 \mathrm{~min}$ before the reaction was stopped by adding $11 \%$ of the volume of $2 \mathrm{M}$ glycine solution. After $5 \mathrm{~min}$ incubation, the cells were washed twice with ice-cold PBS and resuspended in $750 \mu$ FA-lysis buffer (50 mM HEPES, $140 \mathrm{mM}$ $\mathrm{NaCl}, 1 \mathrm{mM}$ EDTA, $1 \%$ Triton X-100, $0.1 \%$ sodium deoxycholate, $0.1 \%$ SDS, pH 7.5). Cells were lysed for $20 \mathrm{~min}$ on ice. Subsequently, sonication was performed 20 times for $25 \mathrm{sec}$ at $70 \%$ time and $55 \%$ amplitude. A total of $6.75 \mathrm{ml} \mathrm{RIPA}$ buffer (50 mM Tris-HCl, $150 \mathrm{mM} \mathrm{NaCl}, 2 \mathrm{mM}$ EDTA, $1 \%$ IGEPAL CA-630, 0.5 \% sodium deoxycholate $0.1 \%$ SDS, $1 \%$ complete protease inhibitor cocktail (reconstituted), $\mathrm{pH} 8.0$ ) and $60 \mu \mathrm{l}$ of protein-A agarose/salmon sperm DNA were added for a preclearing step of $30 \mathrm{~min}$ with slow rotation. Agarose beads were removed by centrifugation at $700 \mathrm{x}$ g for $1 \mathrm{~min}$. Four hundred $\mu \mathrm{l}$ of each sample were collected as reference input and stored at $-20^{\circ} \mathrm{C}$ until further analysis. Overnight incubation with the antibodies for pull-down was performed in a volume of $1.6 \mathrm{ml}$ each at $4^{\circ} \mathrm{C}$ with constant rotation. The following primary and secondary antibodies were used with 
their corresponding dilutions: anti-STAT1 1p91 (M-23); 1:640, anti-acetyl histone; 1:320, and anti-rabbit immunoglobulin ( $\mathrm{IgG}) ; 1: 320$. Incubation with $5 \mu \mathrm{H}_{2} \mathrm{O}$ was included as a negative control. After $16 \mathrm{~h}$ of incubation, $40 \mu \mathrm{l}$ of protein-A agarose/salmon sperm DNA was diluted 1:2 in TE-buffer and added to the sample. Incubation lasted $1.5 \mathrm{~h}$ at $4^{\circ} \mathrm{C}$ with constant rotation. Beads were centrifuged at $4^{\circ} \mathrm{C}$ and $700 \mathrm{x} \mathrm{g}$ for $1 \mathrm{~min}$. The beads were washed in five consecutive steps with low salt buffer $(20 \mathrm{mM}$ Tris-HCl, $150 \mathrm{mM} \mathrm{NaCl}, 2 \mathrm{mM}$ EDTA, $1 \%$ Triton $\mathrm{X}-100,0.1 \%$ SDS, pH 8.1), high salt buffer (20 mM Tris-HCl, $500 \mathrm{mM}$ $\mathrm{NaCl}, 2$ mM EDTA, 1 \% Triton X-100, $0.1 \%$ SDS, pH 8.1), LiCl buffer (10 mM Tris-HCl, $250 \mathrm{mM} \mathrm{LiCl}, 1 \mathrm{mM}$ EDTA, $1 \%$ sodium deoxycholate, 1 \% IGEPAL CA-630, pH 8.1), and TE buffer twice. The DNA/protein complex was eluted with a 1:1 mix of $2 \%$ SDS solution and $200 \mathrm{mM} \mathrm{NaHCO} 3$ solution in two consecutive steps in a total volume of $400 \mu \mathrm{l}$. Afterwards, $14 \mu \mathrm{l}$ of $5 \mathrm{M} \mathrm{NaCl}$ solution were added and incubated at $65^{\circ} \mathrm{C}$ overnight with constant agitation. To each reaction, $7 \mu \mathrm{l}$ of $0.5 \mathrm{M}$ EDTA solution, $14 \mu \mathrm{l}$ of $1 \mathrm{M}$ Tris-HCl solution and $1 \mu \mathrm{l}$ of proteinase $\mathrm{K}$ solution $(20 \mathrm{mg} / \mathrm{ml})$ were added and incubated with the sample for $1 \mathrm{~h}$ at $37^{\circ} \mathrm{C}$. The enzyme was heat-inactivated for $20 \mathrm{~min}$ at $75^{\circ} \mathrm{C}$. DNA was isolated using $360 \mu \mathrm{l}$ phenol/chloroform/isoamyl alcohol (25:24:1). The phases were separated at 10,000 x g for $2 \mathrm{~min}$. The aqueous phase was collected and supplemented with $35 \mu \mathrm{l}$ of $3 \mathrm{M}$ sodium acetate solution and DNA was precipitated by incubation with $800 \mu 1$ isopropanol at $-20^{\circ} \mathrm{C}$ for $1 \mathrm{~h}$. The DNA was collected by centrifugation for $15 \mathrm{~min}$ at $16,100 \mathrm{x} \mathrm{g}$ and $4^{\circ} \mathrm{C}$. The pellet was washed once in $70 \%$ ethanol and left to air-dry before being dissolved in DNase-, RNase- and proteinase-free water. ChIP samples were stored at $-20^{\circ} \mathrm{C}$.

\subsubsection{ChIP-analysis PCR}

ChIP analysis was performed using classical PCR. For the reaction, innuTaq HOT-A DNA polymerase was used according to the manufacturer's recommendations. The reaction included $2.5 \mu \mathrm{l}$ of $10 \mathrm{x}$ buffer containing $\mathrm{MgCl}_{2}, 1 \mu \mathrm{l}$ of $10 \mathrm{mM}$ forward and reverse primer each, $0.625 \mu \mathrm{l}$ of dNTPs $(10 \mathrm{mM}), 0.15 \mu \mathrm{l}$ of polymerase, $1.5 \mu 1$ of DNA and 18.225 of $\mu 1$ DNase-, RNase- and proteinase-free water. The reaction was transferred to a thermocycler and run using the following protocol: $95^{\circ} \mathrm{C}$ for $4 \mathrm{~min}$, followed by 38 cycles of denaturation at $95^{\circ} \mathrm{C}$ for $45 \mathrm{~s}$, annealing at $61^{\circ} \mathrm{C}$ for $45 \mathrm{~s}$, and extension at $72^{\circ} \mathrm{C}$ for $120 \mathrm{sec}$ with a final elongation at $72^{\circ} \mathrm{C}$ for $10 \mathrm{~min}$. The resulting PCR product was run on a $1 \%$ agarose gel at $85 \mathrm{~mA}$ for $45 \mathrm{~min}$ and analysed using the UVsolo TS gel imaging system. 


\subsubsection{RNA analysis}

\subsubsection{RNA extraction from cell culture}

For the extraction of RNA, the PeqGold total RNA kit was used according to the manufacturer's recommendation. Briefly, cells were cultured in starvation medium for $16 \mathrm{~h}$ and subsequently treated for the respective duration of time with the indicated cytokine. At the end of the incubation time, the cells were washed with PBS. Four hundred $\mu 1$ of lysis buffer $\mathrm{T}$ were added to each well. The lysed cells were transferred to the DNA-removing column and centrifuged at $12,000 \mathrm{x}$ g for $1 \mathrm{~min}$. The eluate was mixed with an equal volume of $70 \%$ ethanol and transferred to an RNA-binding column. The column was centrifuged for $1 \mathrm{~min}$ at $10,000 \mathrm{x} \mathrm{g}$, washed once with wash buffer 1 and twice with ethanol-supplemented wash buffer 2. The column was then centrifuged for 1 min to dry at $10,000 \mathrm{x} \mathrm{g}$ and $50 \mu 1$ of DNase-, RNase- and proteinase-free water were added and incubated with the column for $3 \mathrm{~min}$. The RNA was eluted during a 1 min centrifugation at 5,000 x g. The resulting RNA was used directly for cDNA synthesis or stored at $-80 \mathrm{C}$.

\subsubsection{Reverse transcription of RNA isolated using the PeqGold total RNA kit}

To obtain cDNA from the isolated RNA samples, the RNA was reversely transcribed using the Verso cDNA Synthesis Kit from Thermo Fisher according to the manufacturer's instructions. For each sample, $4 \mu \mathrm{l}$ of 5x cDNA synthesis buffer, $2 \mu 1$ of dNTP mix, $1 \mu 1$ of RNA primer (anchored Oligo dT), $1 \mu$ l of RT Enhancer, $1 \mu$ l of Verso Enzyme Mix and $3 \mu 1$ of DNase-, RNase- and proteinase-free $\mathrm{H}_{2} \mathrm{O}$ were mixed and added to $8 \mu \mathrm{l}$ of the purified RNA. The amount of RNA necessary for sufficient yield of the cDNA synthesis had been determined empirically. The mix was transferred to the thermocycler and incubated for $30 \mathrm{~min}$ at $42^{\circ} \mathrm{C}$. The reaction was stopped by heating the sample to $95^{\circ} \mathrm{C}$ for the duration of 2 min. The synthesised cDNA was stored at $-20^{\circ} \mathrm{C}$ until use.

\subsubsection{Real-time PCR}

Endogenous gene transcription was analysed using quantitative PCR (qPCR) technique. The reactions were performed at $95^{\circ} \mathrm{C}$ for 4 min, followed by 38 cycles of denaturation at $95^{\circ} \mathrm{C}$ for $45 \mathrm{~s}$, annealing at $61^{\circ} \mathrm{C}$ for $45 \mathrm{~s}$, and extension at $72^{\circ} \mathrm{C}$ for $120 \mathrm{sec}$ with a final elongation at $72{ }^{\circ} \mathrm{C}$ for $10 \mathrm{~min}$ and the epgradient $\mathrm{S}$ real-time cycler. All primers used in qPCR were diluted to a working concentration of $5 \mu \mathrm{mol} / 1$. A mastermix was prepared containing $10 \mu \mathrm{lof}$ SYBR green, $0.28 \mu \mathrm{l}$ of each primer and $9.44 \mu \mathrm{l}$ of $\mathrm{H}_{2} \mathrm{O}$ for each sample and $19 \mu \mathrm{l}$ of this mix were added to each cavity of a ThermoFast semi-skirted 96-well-plate. One $\mu 1$ of the cDNA 
solution was added to the reaction mix. Each reaction was performed in duplicate. The reaction was run with settings adjusted to Filter $520 \mathrm{~nm}$ 'SYBR', Filter $550 \mathrm{~nm}$ 'X', Sample Vol. '20 $\mu 1$ ', Probe 'SYBR Green'. The following program was used: $95^{\circ} \mathrm{C}$ for $15 \mathrm{~min}$, followed by 45 cycles of denaturation at $95^{\circ} \mathrm{C}$ for $15 \mathrm{sec}$, annealing at $55^{\circ} \mathrm{C}$ for $30 \mathrm{sec}$, and extension at $72^{\circ} \mathrm{C}$ for $30 \mathrm{sec}$. GAPDH was used as reference housekeeping gene. The program realplex ep was used to operate and adjust the settings of the real-time cycler. Data analysis was performed using the $2^{-\Delta \Delta \mathrm{C}_{\mathrm{T}}}$ method (Livak and Schmittgen, 2001).

\subsubsection{Primary cell isolation}

\subsubsection{Isolation of bone marrow cells}

Primary murine bone marrow cells were isolated from mouse femur and tibia. The mice were sacrificed by $\mathrm{CO}_{2}$ inhalation. The fur, skin, muscle and fat were removed from the leg. The leg was cut at the hip joint, the femur separated from the hip bone through dislocation. The foot was removed, femur and tibia were separated from each other using a scalpel cut at the patella. The bones were washed in PBS, and potentially remaining tissue was removed using gauze drenched in PBS. The femurs and tibias were transferred to a cell-culture hood and sterilized for $15 \mathrm{sec}$ in $70 \%$ ethanol. Samples were then rinsed in PBS and transferred to DMEM supplemented with $10 \% \mathrm{FBS}$ and $1 \%$ penicillin/streptomycin for short-time storage. The ends of the bones were cut using sterile surgical scissors. A syringe filled with medium carrying a $21 \mathrm{G}$ needle was inserted into the bone and used to flush the bone marrow into a $50 \mathrm{ml}$ falcon tube. The bone marrow was dispersed using a $23 \mathrm{G}$ needle, passed through a $40 \mu \mathrm{m}$ cell strainer and centrifuged for $5 \mathrm{~min}$ at $100 \mathrm{x}$ g. The supernatant was discarded, and the cells were resuspended in medium at the desired density.

\subsubsection{Isolation of splenocytes and $\mathrm{T}$ cells}

Primary cells from spleen and lymph nodes were obtained from mice which had been immunized with $\mathrm{MOG}_{35-55}$, in order to assess proliferation and production of cytokines. The mice were euthanized using $\mathrm{CO}_{2}$, and the spleen as well as the inguinal and mesenteric lymph nodes were collected in ice-cold preservation medium (EH medium) (DMEM supplemented with glutamine and $25 \mathrm{mM}$ HEPES). The organs were pressed trough a steel mesh to separate the cells and eliminate connective tissue. The resuspended cells were centrifuged at $100 \mathrm{xg}$ for $6 \mathrm{~min}$ at $4^{\circ} \mathrm{C}$, and the pellet was washed once using $\mathrm{EH}$ medium. The cells were resuspended in ReMed. Cell numbers were counted using a Neubauer chamber and the cell suspension diluted for seeding in proliferation and cytokine production assays. 


\subsubsection{Isolation of leukocytes from blood}

To analyse the composition of leukocyte subsets in circulation, $100 \mu 1$ of blood were taken from the retro-orbital sinus of mice using a glass capillary. The blood-filled capillary was transferred to a $1.5 \mathrm{ml}$ reaction tube containing $400 \mu \mathrm{l}$ of PBS/EDTA ( $1 \mathrm{mM}$ EDTA in PBS) solution. Lymphocyte separation medium was carefully added as a sublayer and the samples were centrifuged for $20 \mathrm{~min}$ at $300 \mathrm{x}$ g. The resulting buffy coat $(\sim 200 \mu \mathrm{l})$ was collected, transferred to $3 \mathrm{ml}$ FACS buffer ( $2 \% \mathrm{BSA}, 308 \mathrm{mM} \mathrm{NaN}_{3}$ in PBS) and used for further analysis.

\subsubsection{Assessment of proliferation capacity}

To evaluate whether primary cells derived from spleen and lymph nodes of $\mathrm{MOG}_{35-55}$-immunized animals were able to proliferate upon an antigen stimulus, a proliferation assay was performed. The proliferation rate was assessed based on detection of $\left[{ }^{3} \mathrm{H}\right]$-thymidine incorporated into the DNA. To this end, cells isolated from treated mice as described above were seeded in U-bottom 96-well plates in $100 \mu 1$ ReMed per well. Cell numbers were $3 \times 10^{5}$ for cells obtained from lymph nodes and $6 \times 10^{5}$ for spleenocytes. $\mathrm{MOG}_{35-55}$ at a concentration of $20 \mu \mathrm{g} / \mathrm{ml}$ and, as a positive control, concanavalin $\mathrm{A}$ at a concentration of $1.25 \mu \mathrm{g} / \mathrm{ml}$ were incubated with the samples at $37^{\circ} \mathrm{C}$ and $5 \% \mathrm{CO}_{2}$. Each condition was analysed in triplicate individually for every animal. After $48 \mathrm{~h},\left[{ }^{3} \mathrm{H}\right]$-thymidine was added to a final decay rate of $685.19 \mathrm{~Bq} / \mathrm{ml}$ culture supernatant and incubated with the cells for $16 \mathrm{~h}$. For quantification of $\left[{ }^{3} \mathrm{H}\right]$-thymidine incorporation, cells were lysed and then transferred to a Filter Mat A using the Filter Mate cell harvester. Filter mats were washed six times with water and dried at $80^{\circ} \mathrm{C}$ for $90 \mathrm{~min}$. A MeltiLex scintillator sheet was melted onto each filter mat. The mats were cooled to room temperature and radioactive decay was measured using the MicroBeta TriLux luminescence counter.

\subsubsection{Animal experiments}

\subsubsection{Immunization with $\mathrm{MOG}_{35-55}$ peptide and treatment with LPS}

For the induction of EAE, $\mathrm{MOG}_{35-55}$ peptide mixed with $\mathrm{CFA}$ was used. Mice were anaesthetized using a combination narcotic of $8 \%$ ketamine and $4 \%$ xylazine hydrochloride, in sterile $0.9 \% \mathrm{NaCl}$. The animals were injected with $100 \mu 1$ of $\mathrm{MOG}_{35-55}$ immunization paste (50\% $\mathrm{MOG}_{35-55}$ peptide, $50 \% \mathrm{CFA}$ ), mixed and incubated at $4{ }^{\circ} \mathrm{C}$ for $90 \mathrm{~min}$ prior to injection) subcutaneously in two different sites. Two hundred $\mu 1$ of pertussis toxin solution were applied intraperitoneal (i.p.), and mice were observed while recovering from narcosis. 
Forty-eight hours after immunization with $\mathrm{MOG}_{35-55}$ immunization paste, the animals were injected with a second bolus of $200 \mu 1$ pertussis toxin solution. Lipopolysaccharide (LPS) was injected i.p. $24 \mathrm{~h}$ after immunization with $\mathrm{MOG}_{35-55}$ immunization paste at a concentration of $5 \mu \mathrm{g} / \mathrm{g}$ body weight. Controls were injected with $0.9 \% \mathrm{NaCl}$ solution.

\subsubsection{Clinical scores of EAE}

Beginning on day 10 following immunization with $\mathrm{MOG}_{35-55}$ peptide, mice were observed daily to evaluate EAE disease progression. Scores were assigned following the parameters stated in Table 19. With a score of 4 and above, mice were offered powdered food and water at the bottom of the cage, with a score of 5 and above, mice were additionally kept on cotton mats to avoid sores. Upon reaching a score of 7 or higher or after losing more than $20 \%$ of their original body weight, mice were euthanized. EAE experiments were approved by the LAVES (reference number 3392 42502-04-13/1227).

Table 19: Clinical scores for EAE

\begin{tabular}{ll}
\hline Score & Clinical signs \\
\hline 0 & No clinical signs \\
1 & Reduced tail tonicity \\
2 & Flaccid tail paresis \\
3 & Loss of righting reflex \\
4 & Kinetic ataxia \\
5 & Mild paresis of hind legs \\
6 & Full paresis of one hind leg or partial paresis of both hind legs \\
7 & Paraplegia with full paresis of both hind legs \\
8 & Tetraparesis with (mild) paresis of the forelegs \\
9 & Moribund \\
10 & Dead
\end{tabular}

\subsubsection{Surgery for myocardial infarction}

Myocardial infarction was surgically introduced into STAT1-F77A knockin mice on a C57BL/6N background. Surgery was performed on healthy young adult animals at the age of 8-12 weeks. Three days before surgery, the drinking water of the animals was supplemented with $2 \mathrm{mg} / \mathrm{ml}$ metamizol to provide basic analgesia and to prevent avoidance of metamizol supplemented water after surgery. Mice were anaesthetized using $10 \mu \mathrm{l} / \mathrm{g}$ body weight 
narcotic solution (5\% Cepetor, $10 \%$ midazolam, $10 \%$ fentanyl, in $0.9 \% \mathrm{NaCl}$ ) i.p. and fixed in the supine position. Through a small incision of the trachea, a tube was inserted to provide artificial respiration. Respiration was set at $150 \mu \mathrm{l}$ volume and 150 strokes/min. The skin on the chest was dissected with a lateral cut, the pectoral muscles were detached to expose the rib cage and the $4^{\text {th }}$ intercostal space was spread to expose the heart. The infarction was introduced by ligation of the left anterior descending artery using Ethilon 9-0 BV-4 $5.0 \mathrm{~mm}$ 3/8c EH7448G surgical silk. Whitening of the cardiac apex was observed to control proper occlusion of the coronary vessel. After successful introduction of the infarction, retractors were removed and the skin was sutured using Prolene 6-0 C-1 $13 \mathrm{~mm} \mathrm{3/8c} \mathrm{8889H}$ surgical silk. Narcotic antagonist (5\% Revertor, $10 \%$ Flumazenil, in sterile $0.9 \% \mathrm{NaCl}$ ) was injected i.p. at a dose of $10 \mu \mathrm{l} / \mathrm{g}$ body weight. Mice were given a buprenorphine solution (1\% Temgesic in sterile $0.9 \% \mathrm{NaCl}$ ) at a dose of $15 \mu \mathrm{l} / \mathrm{g}$ body weight and left on a pre-warmed plate at $37^{\circ} \mathrm{C}$ until fully awake. Sham-operated mice underwent the same surgery but the suture around the coronary vessel was removed without occlusion of the left anterior descending artery. After surgery, gavage of metamizol through drinking water was continued. If analgesia was not sufficient, the mice were given buprenorphine (10 $\mu 1 / \mathrm{g}$ body weight) i.p. twice per day. Myocardial infarction experiments were approved by the LAVES (reference number 3392 42502-04-13/1226).

\subsubsection{Echocardiographical assessment}

Transthoracic echocardiography was performed $\leq 7$ days before myocardial infarction (MI) surgery and 3 days after surgery for the acute phase testing and at 1 week and 4 weeks after surgery for the long-term observation cohort. Echocardiography was performed under $1.5 \%$ isoflurane narcosis using the Vevo x2100 system equipped with a $30 \mathrm{MHz}$ centre frequency ultrasound transducer. The long axis was visualized in B mode only, the short axis in B- and M-mode. Heart rates varied in the range of 320-450 bpm, respiration rates ranged from 100150 per minute. Data analysis was performed using the Vevo 2100 software. In the long axis, the diastolic and systolic length was determined $\left(\mathrm{L}_{\mathrm{s}}\right.$ and $\left.\mathrm{L}_{\mathrm{d}}\right)$. In the short axis, left ventricular inner diameter $\left(\mathrm{LVID}_{\mathrm{s}}\right.$ and $\left.\mathrm{LVID}_{\mathrm{d}}\right)$, anterior and posterior wall thickness and left ventricular interior area $\left(\mathrm{Area}_{\mathrm{s}}\right.$ and $\left.\mathrm{Area}_{\mathrm{d}}\right)$ were measured in systole and diastole. In addition, the left ventricular exterior area was measured in systole only. From these data, fractional area shortening (FAS), fractional shortening (FS), ejection fraction (EF), end-diastolic $\left(\mathrm{Vol}_{\mathrm{d}}\right)$ and end-systolic volume $\left(\mathrm{Vol}_{\mathrm{s}}\right)$ were calculated. 
Table 20: Endpoint calculation of echocardiography data

\begin{tabular}{ll}
\hline Measurement & Calculation \\
\hline FAS & $\left(\right.$ Area $_{d}-$ Area $\left._{\mathrm{s}}\right) /$ Area $_{\mathrm{d}} * 100$ \\
$\mathrm{FS}$ & $\left(\mathrm{LVID}_{\mathrm{d}}-\mathrm{LVID}_{\mathrm{s}}\right) / \mathrm{LVID}_{\mathrm{d}} * 100$ \\
$\mathrm{Vol}_{\mathrm{s}}$ & $(5 / 6) *\left(\mathrm{Area}_{\mathrm{s}} * \mathrm{~L}_{\mathrm{s}}\right)$ \\
$\mathrm{Vol}_{\mathrm{d}}$ & $(5 / 6) *\left(\mathrm{Area}_{\mathrm{d}} * \mathrm{~L}_{\mathrm{d}}\right)$ \\
$\mathrm{EF}$ & $\left(\mathrm{Vol}_{\mathrm{d}}-\mathrm{Vol}_{\mathrm{s}}\right) / \mathrm{Vol}_{\mathrm{d}} * 100$
\end{tabular}

\subsubsection{Classical histology}

\subsubsection{Paraffin embedding of tissue and organs}

In order to prepare murine tissue and organs for histological and immunohistological analysis, organs were embedded in paraffin. Mice were sacrificed through cervical dislocation under isoflurane narcosis. Organs of interest were surgically removed and washed in PBS before being perfused for $96 \mathrm{~h}$ in $4 \%$ formaldehyde solution at $4^{\circ} \mathrm{C}$. Organs and tissue were transferred to pure PBS for $16 \mathrm{~h}$ and dehydrated using consecutive ascending concentrations of ethanol, pure xylene and finally paraffin. Paraffin perfused organs were cast in blocks using the EG1160 tissue embedder. To perform histological analysis, paraffin blocks were cut using the SM $2000 \mathrm{R}$ microtome, stretched using a water bath at $45^{\circ} \mathrm{C}$ and dried at $50^{\circ} \mathrm{C}$. Sections were of $5 \mu \mathrm{m}$ in thickness.

\subsubsection{H\&E staining of paraffin embedded sections of murine heart}

To gain an overall impression of tissue conditions, haemalum and eosin (H\&E) staining was performed on paraffin embedded sections. For this purpose, tissue samples were first deparaffinized and re-hydrated by incubation in xylene for twice $15 \mathrm{~min}$ and descending concentrations of ethanol $\left(99 \%, 96 \%, 70 \%\right.$, desalted $\left.\mathrm{H}_{2} \mathrm{O}\right)$ twice for 2 min each. Slides were stained with filtered Mayer's haemalum solution for $10 \mathrm{~min}$ and blued using running tapwater for $10 \mathrm{~min}$. The slides were counterstained in $0.1 \%$ eosin solution for $2 \mathrm{~min}$, washed twice in desalted water and dehydrated using ascending concentrations of ethanol (70 \%, $96 \%, 99 \%)$ twice for $2 \mathrm{~min}$ each followed by two $15 \mathrm{~min}$ incubation steps in xylene. The samples were mounted using Entellan and analysed using light microscopy. 


\subsubsection{Immune staining techniques}

\subsubsection{Fluorescence-activated cell sorting analysis}

When performing fluorescence-activated cell sorting (FACS) analysis, cells of spleen, lymph nodes or circulating leukocytes were stained after producing a single cell suspension. If nothing else is stated, $5 \times 10^{5}$ cells were used for analysis. The cells were centrifuged at $185 \mathrm{x}$ $\mathrm{g}$ for $8 \mathrm{~min}$ and $4^{\circ} \mathrm{C}$ and washed once with FACS buffer. They were stained after resuspension in $100 \mu \mathrm{l}$ antibody mastermix (FACS buffer with antibodies) for $15 \mathrm{~min}$ at $4^{\circ} \mathrm{C}$ while being protected from light. Cells were washed again once using FACS buffer and were resuspended in $1 \mathrm{ml}$ FACS buffer before analysis. Staining of nuclear forkhead box P3 (FoxP3) was performed using, the PE anti-mouse/rat/human FOXP3 Flow kit according to the manufacturer's recommendations. After having been stained for extracellular markers, the cells were incubated for $30 \mathrm{~min}$ with $400 \mu \mathrm{l}$ Fix-Perm solution, protected from light throughout the entire staining. Cells were washed once in FACS buffer. Blocking was performed for 15 min in $2 \%$ rat serum in perm buffer. One $\mu 1$ of FoxP3 antibody was then directly added and incubated with the cells for $30 \mathrm{~min}$. Cells were washed twice in perm buffer and resuspended in $200 \mu \mathrm{l}$ FACS buffer.

Sorting was performed using the FACSCalibur cell sorter. For analysis, the software CellQuestPro was used. A minimum of 30,000 cells was sorted for quantitative analysis. Four different reaction mixes staining for different leukocyte subsets were used for detection. One mix was targeted to identify different $\mathrm{T}$ cell-subsets and contained CD4 PE e610 (1:500), CD8 PE/Cy7 (1:333), CD44 PE (1:333), and CD62L (1:200). In a second reaction, myeloid cell subsets were identified using CD11b FITC (1:200), Ly-6C APC/Cy7 (1:500), CCR2 AF700 (1:20), and Ly-6G PE (1:1,000). The third staining was performed to identify NK and NKT cells using TCR $\beta$ FITC (1:200), CD4 PE (1:1,000), and NK-1.1 APC/Cy7 (1:250), and in the fourth reaction, regulatory $\mathrm{T}$ cells were quantified using CD25 FITC (1:200), CD4 PE/Cy5 (1:500) and the PE anti-mouse/rat/human FOXP3 Flow kit.

\subsubsection{Immune staining using HRP-reaction}

Immune staining for the detection of the protein localisation in tissue sections was performed using a peroxidase reaction. The tissue sections were de-paraffinized and re-hydrated by incubation in xylene twice for 15 min followed by a series of descending concentrations of ethanol $\left(99 \%, 96 \%, 70 \%\right.$, desalted $\left.\mathrm{H}_{2} \mathrm{O}\right)$ twice for 2 min each. Slides were boiled in citrate buffer ( $8.13 \mathrm{mM}$ sodium citrate, $\mathrm{pH}$ 6.0) for $15 \mathrm{~min}$, left to cool on ice and rinsed with double-distilled $\mathrm{H}_{2} \mathrm{O}$ and PBS. A peroxidase block was performed using $3 \% \mathrm{H}_{2} \mathrm{O}_{2}$ in PBS for 
20 min at $4{ }^{\circ} \mathrm{C}$. The tissue was blocked in $10 \%$ FBS in PBS supplemented with $0.05 \%$ Triton $\mathrm{X}-100$ and incubated with the primary antibody in $10 \% \mathrm{FBS}$ in PBS for $16 \mathrm{~h}$ at $4^{\circ} \mathrm{C}$. The antibody was removed and the tissue rinsed three times with PBS before the biotinylated secondary antibody diluted 1:200 in 10\% FBS in PBS was applied for $1 \mathrm{~h}$ at room temperature. After rinsing the slide four times with PBS, it was incubated with streptavidinperoxidase diluted 1:1,000 in PBS for $1 \mathrm{~h}$. Slides were developed using DAB. The $2.5 \%$ stock solution of DAB was diluted 1:250 in PBS supplemented with $0.012 \% \mathrm{H}_{2} \mathrm{O}_{2}$ and incubated with the tissue until the chromatic reaction was clearly visible. The reaction was stopped by rinsing off the DAB with double-distilled $\mathrm{H}_{2} \mathrm{O}$. Nuclei were counterstained with Mayer's haemalum solution for $10 \mathrm{~min}$ and blued using running tap water for $10 \mathrm{~min}$. The slides were washed twice in desalted water and dehydrated using ascending concentrations of ethanol $(70 \%, 96 \%, 99 \%)$ twice for 2 min each and two 15 min incubation steps in xylene. The samples were mounted using Entellan and analysed using light microscopy. Dilutions of primary antibodies were as follows: anti-phosphotyrosine-STAT1; 1:200, antimyeloperoxidase (MPO); 1:200, anti-CD68; 1:75, and anti-CD3 1:200.

\subsubsection{Evaluation of staining intensity}

Ventricle myocardial infiltration was assessed using two independent measures, the distribution of cells staining positive and the density of these cells within the positive area. Both measures were scored on a scale from 0-4. The criteria applied for scoring the distribution are listed in Table 21. The criteria applied for scoring the density are listed inTable 22. For the evaluation of the H\&E stained hearts, only the distribution criteria were applied.

Table 21: Criteria for distribution scores

\begin{tabular}{ll}
\hline Score & Characteristics \\
\hline 0 & No specific staining \\
1 & Less than half of the left ventricle \\
2 & $50 \%$ to $90 \%$ of the left ventricle \\
3 & Entire left ventricle but little or no infiltration of the septum or \\
& right ventricle \\
4 & Entire left ventricle and strong infiltration of the right ventricle or \\
& the septum
\end{tabular}


Table 22: Criteria for density scores

\begin{tabular}{ll}
\hline Score & Characteristics \\
\hline 0 & No specific staining \\
1 & Single cells at low density \\
2 & Small accumulations, moderate density \\
3 & Many or large accumulations \\
4 & High, even density of positively stained cells
\end{tabular}

\subsubsection{Statistical analysis}

For statistical analysis, IBM SPSS and SigmaPlot were used. Means and standard deviations or standard errors were calculated from the data. Between-group differences were assessed using Student's $t$-test. A p value of .05 was chosen as level of significance. 


\section{Results}

\subsection{N-terminus-mediated intradimeric interactions are dispensable for protomer exchange kinetics of STAT1}

It is known that STAT1 dimers need to undergo a conformational change from a parallel to an antiparallel alignment to be prone to inactivating dephosphorylation (Zhong et al., 2005). Mertens and colleagues have proposed a mechanism in which intradimeric N-terminal interactions facilitate the rotation of dimer partners from one of the conformational states to the other (Mertens et al., 2006) (Figure 2, model 1). Here, experimental data support an alternative mechanism to achieve conformational reorientation, namely, conversion of parallel to antiparallel STAT1 dimers is achieved through dissociation and re-association of STAT1 protomers (Wenta et al., 2008) (Figure 2, model 2). Kinetics of the dissociation of dimers and tetramers were investigated using EMSA technique, utilising native and GFP-tagged STAT1 protein derived from transfected U3A cells. Furthermore, the question as to whether $\mathrm{N}$ terminal interactions - especially those facilitated by the phenylalanine residue at position 77 - are essential for this process has been addressed.

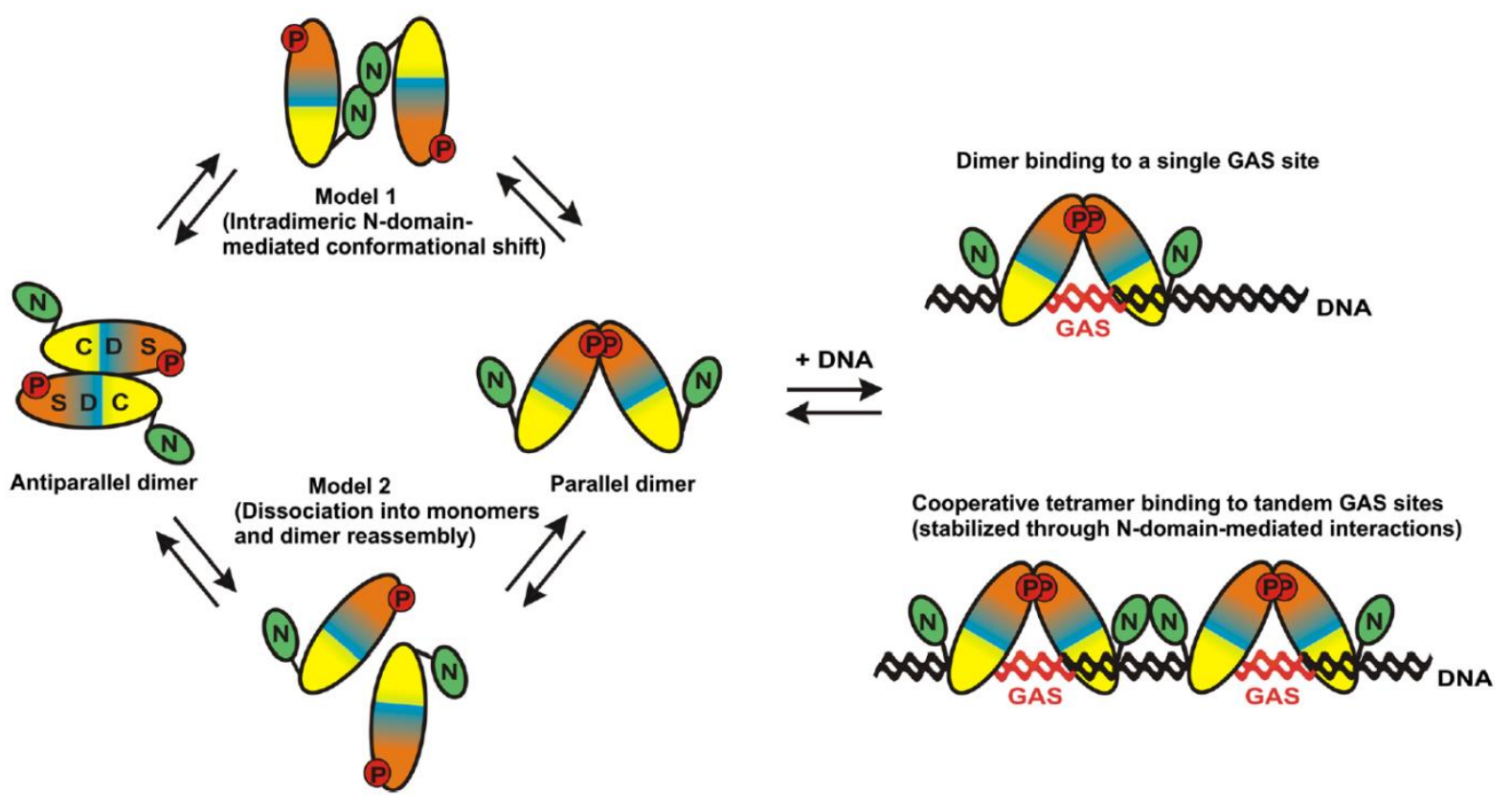

Figure 2: Proposed mechanisms for STAT1 conformational change. Model 1 by Mertens et al,. 2006 suggested that the transition between two conformational states is facilitated through reciprocal $\mathrm{N}$ terminal interactions. Model 2 is based on the dissociation and reassociation of STAT1 dimers. Highaffinity DNA binding (dimeric as well as tetrameric) is only possible in the parallel form. $N=N$ terminus, $C=$ coiled-coil domain, $D=D N A$-binding domain, $S=S H 2$ domain, $P=$ phosphate group . 


\subsubsection{DNA-binding kinetics do not differ among native STAT1 and GFP-tagged STAT1}

In order to assess the exchange kinetics of STAT1 protomers, an experimental system was established. The vectors pcDNA3.1, coding for full-length native STAT1, and pEGFP-N1, encoding a GFP-tagged STAT1 fusion protein, were utilized to introduce two different versions of the STAT1 gene into the STAT1-deficient cell line U3A. These two constructs differ in weight which makes it possible to distinguish them in EMSA readouts and in their ability of subcellular trafficking (Meyer et al., 2007). First, we evaluated whether they also differ with respect to their DNA-binding kinetics which would render them useless for the intended experiments.

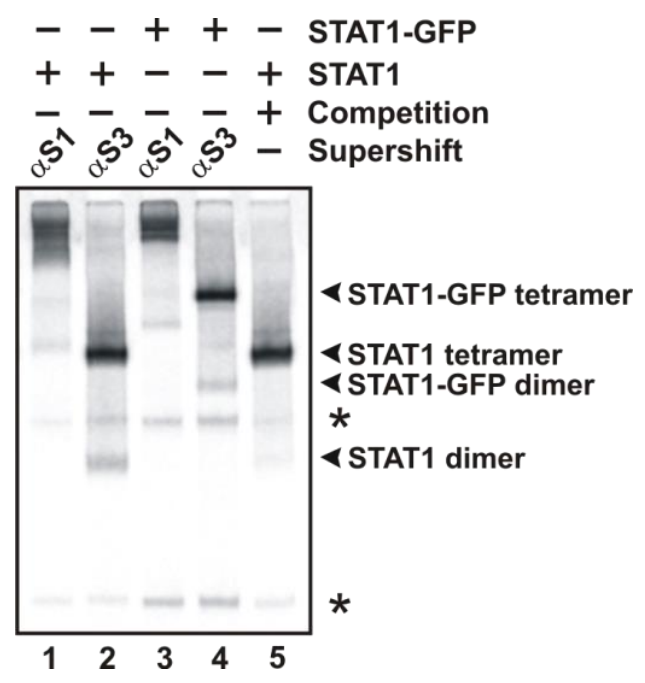

Figure 3: Native STAT1 and STAT1-GFP both favour tetrameric binding to tandem GAS sites. EMSA confirmed the identity of recombinant STAT1 proteins interpreted to be the untagged (lane 1, lane 2 and lane 5) and the GFP-tagged variant (lane 3 and lane 4) using supershift reactions with either anti-STAT1 (lane 1 and lane 3) or anti-STAT3 (lane 2 and lane 4) antibody. Lane 5 shows the result of a competition experiment with unlabelled GAS elements added in 750-fold excess. The asterisk indicates an unspecific band.

Protein extracts derived from U3A cells transfected with a construct carrying either the native STATl gene or the gene for STAT1 with a GFP-tag were incubated with the $\left[{ }^{33} \mathrm{P}\right]$ labelled 2xGAS probe and analysed by means of EMSA (Figure 3). Both STAT1 proteins bound to the probe specifically as dimeric and tetrameric complexes, as shown in lane 2 and lane 4 for native STAT1 and GFP-tagged STAT1, respectively. For both variants, the slower migrating complex corresponding to the tetrameric form was favoured over the dimeric complex, as indicated by stronger signal intensity in the autoradiograms. The specificity of the signal was confirmed by supershift reaction: Addition of a STAT1-specific antibody 
significantly shifted the dimer and tetramer band (lane 1 and 3), whereas an antibody directed against STAT3 was unable to cause this effect (lane 2 and 4). Co-incubation with a 750-fold molar excess of an unlabelled 2xGAS oligonucleotide rapidly weakened the signal for dimeric STAT1 bound to the probe, while leaving the signal intensity for the tetramer almost unaffected. This highlights the higher stability of tetrameric complexes bound to DNA as compared to dimeric complexes - an effect highly dependent on reciprocal interactions between the STAT1 amino-termini (Vinkemeier et al., 1996; Begitt et al., 2014).

A

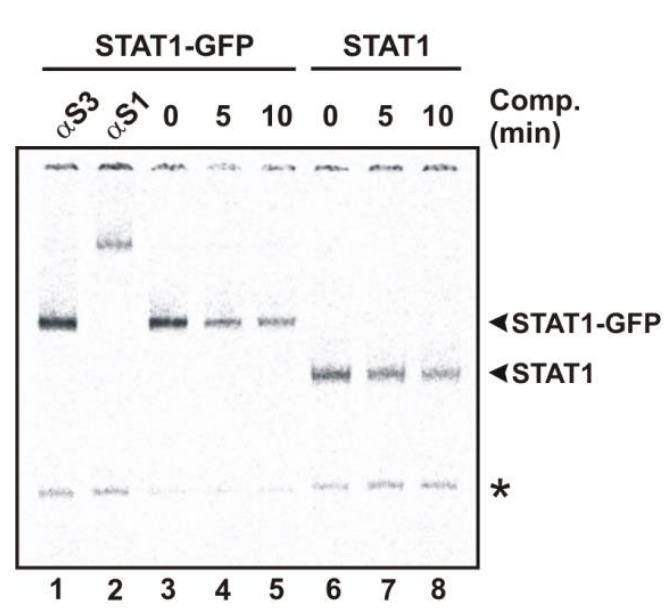

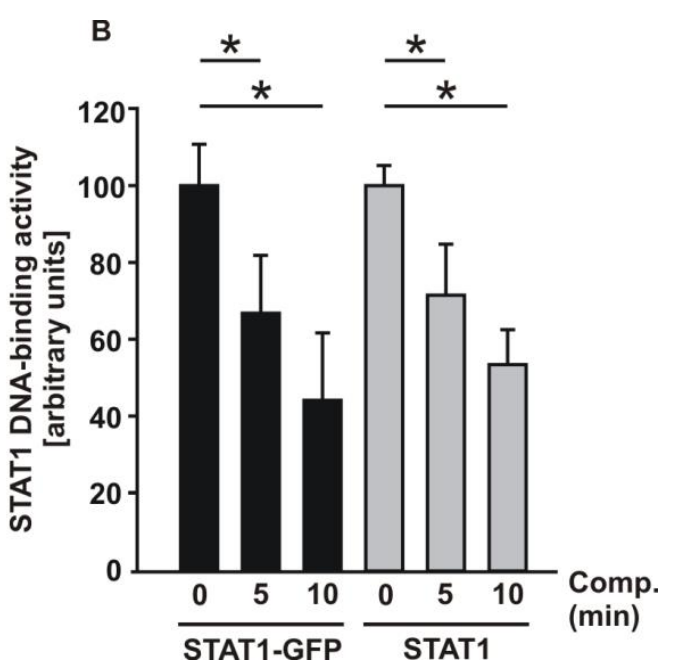

Figure 4: Competition assay showing the dissociation kinetics of STAT1 dimers from single GAS elements. (A) Protein extracts from U3A cells transfected with plasmids coding for either wildtype or GFP-tagged STAT1 were incubated with $\left[{ }^{33} P\right]$-labelled M67 for $15 \mathrm{~min}$ and subsequently coincubated with a 750-fold molar excess of unlabelled M67 duplex for the indicated times. The asterisk indicates an unspecific band. (B) Densitometry of three independent experiments showing the results from the competition assay in A. Error bars represent the standard deviation. Asterisks indicate statistically significant differences.

In order to compare the DNA-release kinetics of the two STAT1 variants, a competition with the untagged M67 oligonucleotide was performed (Figure 4). Protein extracts from IFN $\gamma$-treated U3A cells which had been transiently transfected with a plasmid coding for either native or GFP-tagged STAT1 were incubated on ice for $15 \mathrm{~min}$ with the radioactively labelled M67 probe which contained a single GAS element. A 750-fold molar excess of nonlabelled M67 oligonucleotide was added 0, 5 and $10 \mathrm{~min}$ before the samples were loaded onto the non-denaturing polyacrylamide gel. As seen in lanes 6-8 of Figure 4A, DNA binding of native STAT1 was reduced with time owing to competition from unlabelled DNA. The same effect was observed for the STAT1-GFP molecule (lanes 3-5). Quantitative assessment of three independent experiments demonstrated that DNA-bound STAT1 dimers are released 
relatively rapidly and that the dissociation kinetics of the native and the GFP-tagged STAT1 variants are comparable (Figure 4B).

\subsubsection{Native and GFP-tagged STAT1 form hetero-tetrameric complexes ex vivo}

Given the observation that native and GFP-tagged STAT1 both bind to DNA with similar kinetics, the next step was to observe whether the two variants were able to interact in order to form hetero-tetramers. Extracts from IFN $\gamma$-treated U3A cells expressing either recombinant GFP-tagged or untagged STAT1 were incubated with radioactively labelled 2xGAS probes. As a control, each of the protein variants was incubated with the DNA probe separately for $20 \mathrm{~min}$ on ice and the resulting samples were then combined immediately before being loaded onto the gel (Figure 5, lane 5). In this setting, the two homo-tetramers can be clearly distinguished. To allow interchange of dimers, a separate reaction was set up where the $\left[{ }^{33} \mathrm{P}\right]-$ labelled 2xGAS probe was incubated with a mix of both extracts for 20 min before being loaded onto the gel (Figure 5, lane 6). In this setting of co-incubating the extracts with the probe, both bands corresponding to the homo-tetramers in lane 5 appeared again. Additionally, a third band appeared between the two homo-tetramers which was interpreted as a 2xGAS probe occupied by two homo-dimers each containing either GFP-tagged or untagged STAT1.

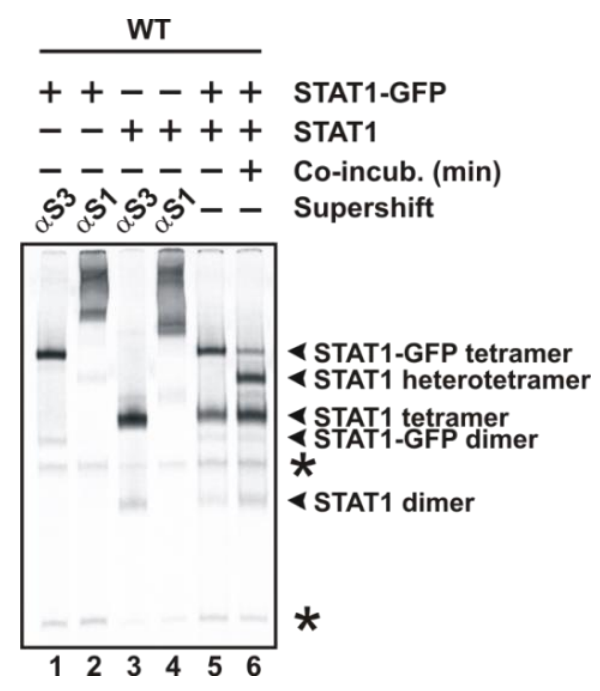

Figure 5: Native STAT1 and STAT1-GFP can form hetero-tetramers. Extracts from U3A cells expressing native or GFP-tagged STAT1 were incubated with the $\left[{ }^{33} P\right]$-labelled $2 x G A S$ probe either separately (lane 5) or mixed together (lane 6) for 20 min. Asterisks indicate unspecific bands. 
To validate the identity of the additional band, a co-incubation of the extracts before loading with varying portions of both STAT1 variants was performed (Figure 6). Lane 1 and 2 correspond to lane 5 and 6 from Figure 5. In lanes 3 and 4, the amount of extracts from cells transfected to produce native STAT1 exceeded the extracts from lane 5 and 6 by the factor 5 , while this relation was reversed for GFP-tagged STAT1. It becomes clear that, in lane 4, there is almost no signal from the band representing the STAT1-GFP homo-tetramer, whereas in lane 6, the signal from the native STAT1 homo-tetramer band was markedly reduced compared with the corresponding band in lane 2 and 5. This strong reduction in the signal intensity indicated that the newly formed tetrameric complex is indeed a hetero-tetramer consisting of two STAT1-GFP/STAT1-GFP and STAT1/STAT1 homodimers, respectively.

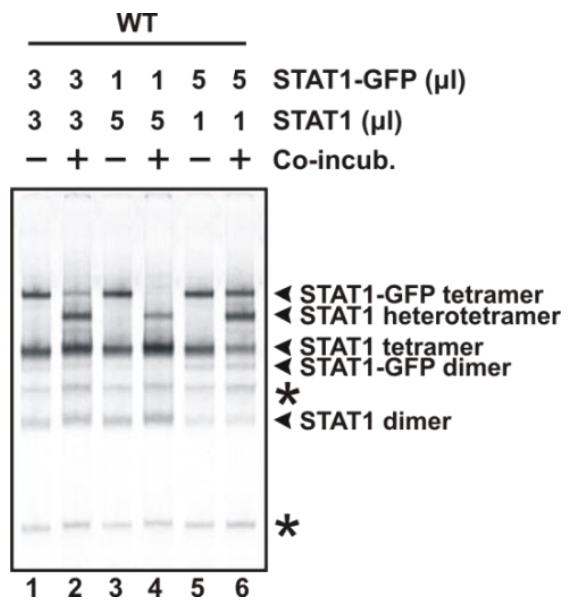

Figure 6: STAT1 hetero-tetramers consisting of two homodimers are formed on tandem GAS sites. Extracts from U3A cells expressing either recombinant, untagged or GFP-tagged STAT1 were coincubated at ratios of 1:1, 1:5 and 5:1 with $\left[{ }^{33} P\right]-2 x G A S$ elements before loading on a non-denaturing EMSA gel. Lanes with odd numbers show the results for extracts that were incubated separately, while lanes with even numbers correspond to the co-incubated extracts. Asterisks indicate unspecific bands.

\subsubsection{The rate of protomer exchange is reduced through binding to DNA}

To this point, the data demonstrated the presence of a STAT1/STAT1-GFP heterotetramer only with a 1:1 stoichiometry. This complex most probably consisted of two homo-dimers since no additional bands representing a $1: 3$ or $3: 1$ stoichiometry were observed. In order to validate the conditions under which complexes with a 1:3 and 3:1 stoichiometry were formed, extracts from IFN $\gamma$-treated U3A cells transfected with either variant were incubated separately in the presence of DNA (Figure 7, lane 1). Alternatively, lysates containing the two variants were mixed in the absence of $\left[{ }^{33} \mathrm{P}\right]-2 \mathrm{xGAS}$, which was only added immediately before the reactions were loaded onto the gel (Figure 7, lane 2). The two reactions were incubated for 
$45 \mathrm{~min}$ at room temperature, before the DNA-bound STAT1 complexes were electrophoretically separated by EMSA. When free exchange of STAT1 molecules was allowed in the absence of co-incubating DNA, this resulted in the occurrence of several additional bands not detected in the presence of the 2xGAS probe. This observation showed that not only dimers but also single tyrosine-phosphorylated protomers can interchange between different dimer pairs in solution, unless STAT1 is bound to high-affinity DNAbinding sites present in the incubation mix.

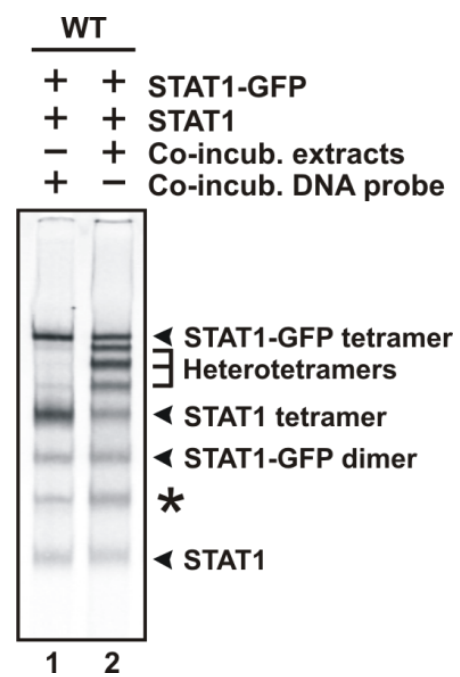

Figure 7: Untagged and GFP-tagged STAT1 form hetero-tetramers with 3:1 and 1:3 stoichiometry when unbound to DNA. Co-incubation of extracts from reconstituted U3A cells expressing either untagged or GFP-tagged STATl at room temperature for 45 min before addition of $\left[{ }^{33} P\right]-2 x G A S$ probe resulted in the appearance of the predicted bands with 3:1 and 1:3 stoichiometry (lane 2). No such bands were detected in the presence of binding to GAS elements. The asterisk indicates an unspecific band.

The kinetics of the promoter exchange was then investigated. Protein extracts from reconstituted U3A cells containing either recombinant untagged or GFP-tagged STAT1 were co-incubated with the 2xGAS DNA probe for different durations of time (Figure 8). A comparison of lane 1 with lane 2 and 3 in Figure 8A shows that longer co-incubation of the STAT1 isoforms in the absence of DNA resulted in increased interchange of dimers and protomers of STAT1. This demonstrates that binding of STAT1 to DNA hinders the replacement of protomers within dimers. 
A

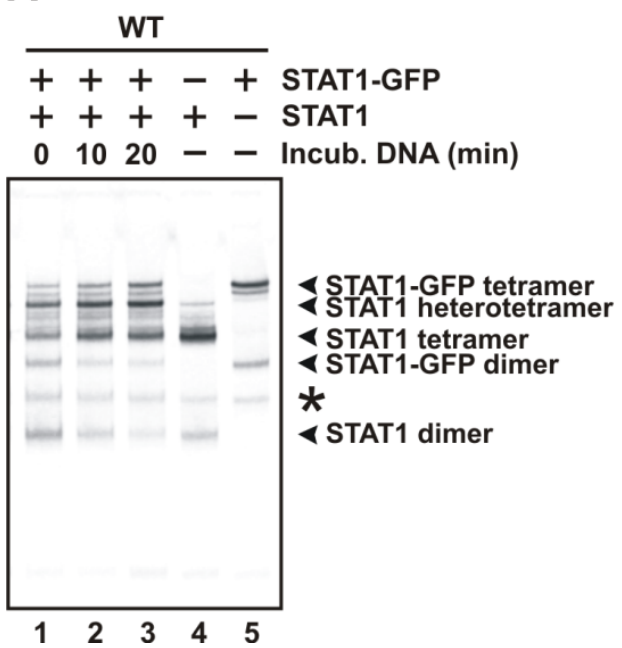

B

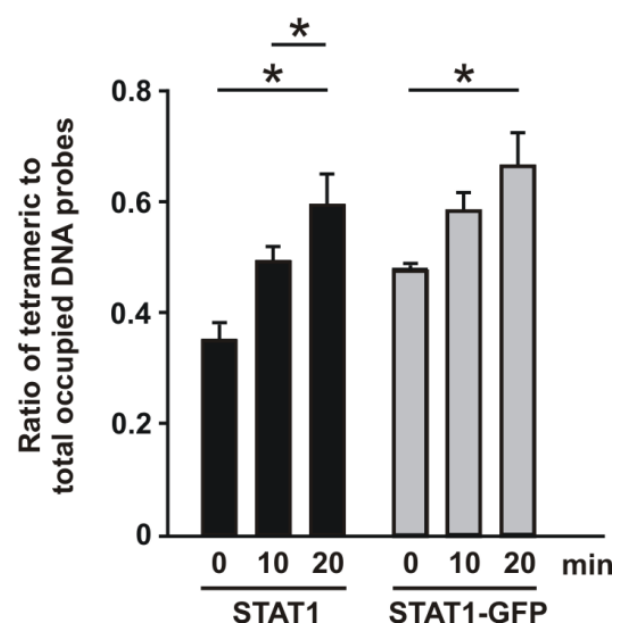

Figure 8: Binding of STAT1 to DNA hinders the exchange of protomers. (A) $\left.{ }^{33} P\right]$-labelled $2 x G A S$ was incubated with mixed extracts from U3A cells expressing either untagged STAT1 or STAT1-GFP for the indicated durations of up to 20 min (lane 1-3). Lane 4 and 5 display the results of $\left[{ }^{33} P\right]-2 x G A S$ incubation with only one of the STAT1 variants. The asterisk indicates an unspecific band. (B) Densitometry from three independent experiments showing the tetramer signal in relation to the combined signal of dimers and tetramers. Error bars represent the standard deviation. Asterisks indicate statistically significant differences.

Furthermore, densitometric analysis of three independent experiments verified the impression that increasing incubation time of STAT1 with DNA favoured the formation of tetrameric complexes over the binding as a dimer. In summary, these data highlight the significance of DNA binding in the dissociation and re-association of dimers and the stabilization of tetrameric STAT1 complexes.

\subsubsection{The exchange of STAT1 protomers is a process independent of intradimeric N- terminal interactions}

To allow for inactivation of tyrosine-phosphorylated STAT1 dimers, a conformational change from a parallel to an antiparallel alignment is necessary (Zhong et al., 2005). A mechanistic model had been proposed by Mertens and colleagues that required intradimeric interactions between the N-termini of the partner protomers to break the phosphotyrosine-SH2 binding (Mertens et al., 2006). As a tool to study N-terminal interactions, the STAT1-F77A mutant was created. By exchange of the phenylalanine at position 77 to alanine, reciprocal N-domainmediated binding between two dimers was critically weakened (Meyer et al., 2004). Further insights into the dynamics of protomer exchange were expected from studying the phenotype of the STAT1-F77A mutant. Since the STAT1-F77A mutant had been shown to display more efficient and prolonged phosphorylation as compared to the wildtype protein (Meyer et al., 
2004), protein extracts used in further experiments were tested by means of Western blotting technique to adjust for equal amounts of tyrosine-phosphorylated STAT1 (Figure 9B).

A

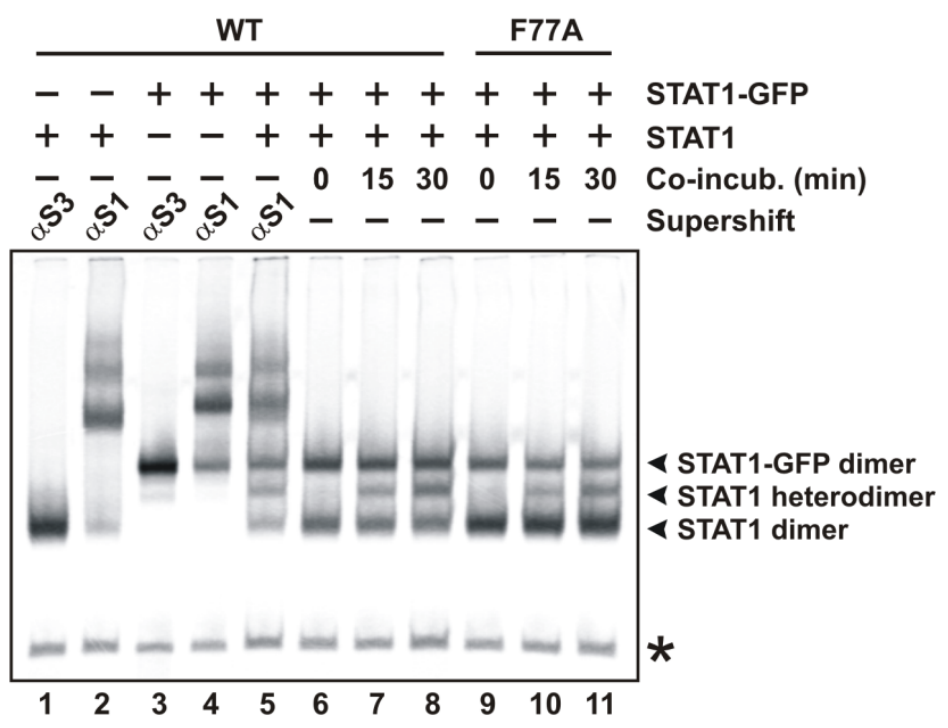

B
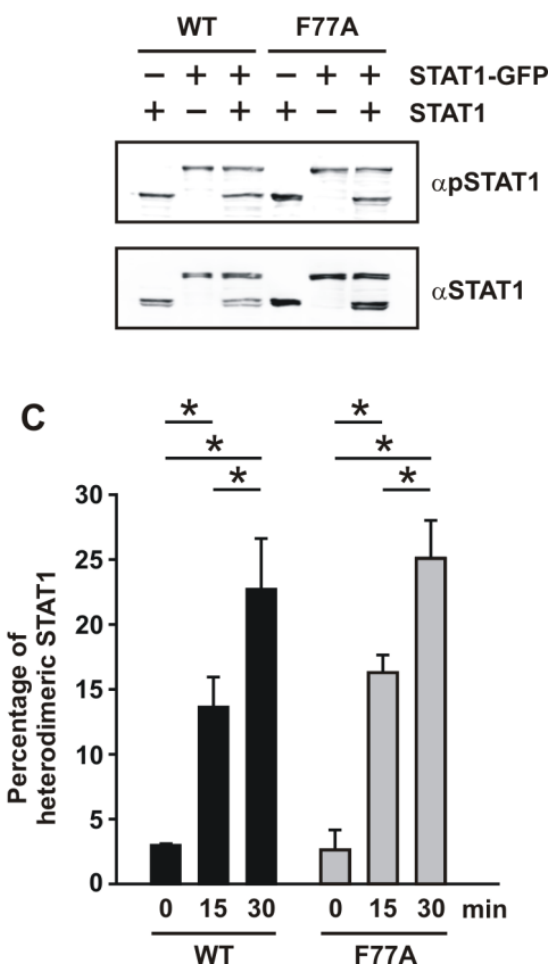

Figure 9: Protomer exchange kinetics of dimers does not differ between the wildtype STAT1 and the F77A mutant. (A) Protein extracts of U3A cells that had been transfected with the native or GFPtagged form of WT or F77A mutant STAT1 were co-incubated for the indicated durations. The probe used for binding STAT1 was $\left[{ }^{33} P\right]-M 67$ containing a single GAS site. The asterisk indicates an unspecific band. (B) No evidence of gross structural alterations in the STAT1-F77A mutant was detected as judged from Western blotting results. Lysates from IFNy-pre-treated, reconstituted U3A cells expressing either the untagged form of STATl $\alpha$ with a molecular weight of $91 \mathrm{kDa}$ or the GFPtagged variant with the molecular weight of $118 \mathrm{kDa}$ were tested in isolation or as a mixture using a STAT1-phospho-tyrosine-specific antibody (top). The result from stripping-off bound immunoreactivity and exposure of the membrane with a pan-STAT1 antibody is shown at the bottom. (C) Densitometric analysis of three independent experiments performed as demonstrated in (A). Shown is the proportion of heterodimers among all dimers that were detected. Error bars represent the standard deviation. Asterisks indicate statistically significant differences.

Protein extracts from transfected U3A cells expressing the untagged or the GFPconjugated isoform of wildtype or F77A mutant STAT1 were co-incubated for 0,15 or 30 min before addition of the M67 DNA probe (Figure 9A). Lanes 6-8 depict a representative result for wildtype STAT1. In line with the above mentioned observations, an increase in STAT1 hetero-dimers with increasing times of co-incubation before addition of the probe was detected. As can be seen from lane 9-11, the pattern of the F77A mutant did not differ from 
the wildtype molecule. The readouts from three independently conducted EMSAs were quantified using densitometry analysis. The results shown in Figure 9C confirmed that there was no significant difference in the intradimeric protomer exchange kinetics between the wildtype protein and the F77A mutant when binding to a DNA probe carrying a single GAS motif was studied.

Subsequently, the differences in the DNA-association kinetics between the wildtype and the F77A mutant were tested by means of EMSA using the 2xGAS probe (Figure 10A). Lanes 7-9 show the results for the wildtype. Lane 7 corresponds to completely separate incubation of the wildtype and the GFP-tagged variant, lane 8 displays the co-incubation of the two isoforms in the presence of GAS, and lane 9 depicts co-incubation of the two STAT1 variants in the absence of GAS elements which were added immediately before loading onto the gel. Again, the results confirmed the conclusion from the previous experiments. Lanes 10-12 show the respective results for the STAT1-F77A mutant. The slight retardation in the migration of the tetramer band of native STAT1 carrying the F77A mutation probably results from a less compact form of the homo-tetramer due to its lack of tetramer stabilization through $\mathrm{N}$ terminal interactions (Vinkemeier et al., 1996; Meyer et al., 2004).

As for the wildtype molecule, little to no signal was detectable for recombined isoforms other than the two homo-tetramers, upon incubation of the two STAT1 variants with the 2xGAS probe separately (Figure 10A, lane 10). Upon incubation of the two isoforms together with the DNA probe for the entire duration of the experiment, all five different compositions of the tetramer are observed (lane 11). When the protein extracts were mixed and coincubated before addition of the DNA, again all five species appeared (lane 12). The main difference between the result of the incubation of the mixed protein extracts with and without the probe is the higher concentration of hetero-tetramers with 3:1 and 1:3 stoichiometry which corroborated the earlier observation that dimers within a tetramer are exchanged at a faster rate than protomers and that exchange rates for dimers and monomers dramatically drop as soon as they are bound to DNA.

In Figure 10B, the results from the corresponding densitometric analysis are displayed. The quantitation of the EMSA results showed the relative amount of GFP-tagged and untagged STAT1 isoforms in the tetramers formed under conditions of co-incubation with protein extracts and/or GAS elements. This analysis underlines the initial observation demonstrating that there is no difference in the protomer exchange kinetics between the STAT1 wildtype 
protein and its F77A mutant. This finding supported the conclusion that reciprocal N-terminal interactions are dispensable for the exchange of protomers.
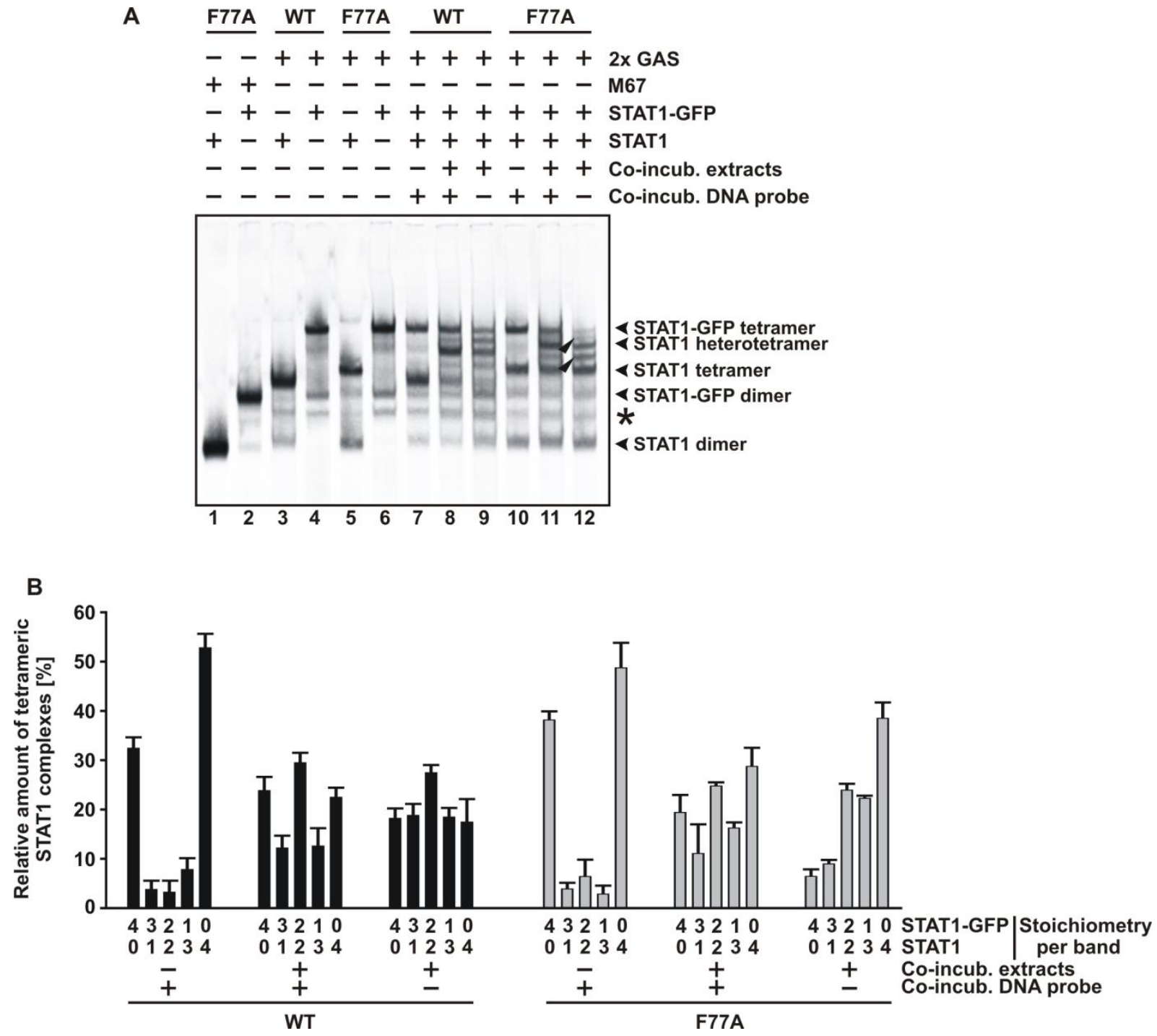

Figure 10: Amino-terminal interactions are dispensable for monomer exchange of STAT1. (A) EMSA experiment comparing the intratetrameric and intradimeric subunit exchange kinetics of wildtype STAT1 (lane 7-9) with its F77A mutant (lane 10-12). The asterisk indicates an unspecific band. Unconjugated wildtype STAT1 homo-tetramers run slightly faster than STAT1-F77A homotetramers probably due to a more condensed structure facilitated through tetramer stabilization. (B) Densitometry analysis of the proportions of each variant at the indicated stoichiometric ratio from three independent gel runs. Error bars represent the standard deviation. Asterisks indicate statistically significant differences. 


\subsection{Gene expression of ezrin is weakly induced by STAT1}

\subsubsection{Unique GAS motif in the promoter region of the human ezrin gene was identified}

Ezrin, radixin and moesin constitute the ERM protein family, a class of cytoskeletal proteins with the main function of linking transmembrane proteins to the actin cortex. While recent studies have focused on the role of ERM proteins on leukocyte recruitment (e.g. Killock et al., 2009; Wang et al., 2014), less is known about the regulation of their gene expression.

An in silico search revealed the presence of a unique STAT-binding element in the promoter of the human ezrin $(E Z R)$ gene approximately 500 bases upstream of the first exon (Table 23). This motif consists of two GAS elements located in tandem orientation which are separated by only one base pair. Each element closely resembles the consensus STAT-binding site, termed GAS sequence (TTC[N] $]_{3-4} \mathrm{GAA}$ ), which is usually located in the vicinity of the transcriptional start site (Decker et al. 1997).

Table 23: GAS motifs surrounding the transcription-start site (TSS) in the promoter of the human EZR gene. Gene sequence was obtained from www.ensembl.org.

\section{>chromosome:GRCh38:6:158765141:158820912:-1}

TTCTAGAGTGAGCTCTCCCGCAGCAAATTCTACTGGCCCCCAAAGTATGTGTTTTGTGTGTCTTAAAA ATTTGTTGAGAACCATTAGCAAAAAAACAAACAAAAAAACTTAA TTCCTA GAATTTCAGA GAAATCCC ATGGAGCTTTTTGCCAGTCACGTCAAAAGAGGCCACAAACGTGCCACTTAACCAGAGCTTCGGAAAGG CGGCGGCTGGGCCGGCCACGTGCACCGAGACTCGGGGCCAGGTGCAGCCGCCCCAGGGCCGAGGCCTC GGAACTGGCCCCCGGTCCCGGCCCCAAGCGGTCCAGCGATTCCCCCAAGCCGTCCGCCCCTCCAGATT TATTTACGTTTTCCTGACTTCCCCCTGCCCGCTGTGGGACAAACAGCCTCCCCACTTGCATCTGCGAG GGGAGTAGCGCGCACTTCCGCCAAGTTCCGCCCCCACCCAGCCCGAGGCCCGGCTGCCGCCATCTTGC GGGGGGCGCACCTCACAGGTCGGGAGCTGGGCGGGAAGGGGCGTGGTCCCGGGACCCGCCCCGCCGGG GCTTTTGGGAGCGCGGGCAGCGAGCGCACTCGGCGGACGCAAGGGCGGCGGGGAGCACACGGAGCACT GCAGGCGCCGGGTGAGGCGTGCGGCGGCCGGGGTC

\section{Exon 1, IFNY-activated site motif}

Predicted TSS using the data base Berkeley Drosophila Genome Project (BDGP) neural network prediction tool (http://www.fruitfly.org/seq_tools/promoter.html)

Given that the IFN $\gamma / \mathrm{STAT} 1$ axis is a signalling pathway highly relevant to a plethora of immunological processes, STAT1 may be a candidate for regulating ezrin signalling in leukocytes. Although in the mouse genome a similar tandem GAS motif cannot be found in the Ezr gene promoter (Table 24), three optimal and two non-optimal GAS motifs were identified in a region spanning 1,200 base pairs upstream of the transcription start site to the 
first intron. This finding warranted further research efforts to investigate the IFN $\gamma /$ STAT1 signalling pathway as a putative mediator of $E z r$ gene transcription in mice.

Table 24: GAS motifs surrounding the TSS of the murine EZR gene sequence. Gene sequence was obtained from www.ensembl.org.

>chromosome:GRCm38:17:6737441:6785784:1

ACCTAGCACGGCACTAATTTCACGTGTGTA TTCATT GAAGTCTACATCTGTGTAGATGTTGCTCCTTG GATCCCTA TTCTACAAATTAGGATTACCGGGTCCCAAAGGAACTTTTCCCATTCTGGAATGGATATAC TCAGCCCCGGAACGGAGTTGTGCACATGTCACATTCAATACATCTAATCCTCATTACAACTCACGGAA CAGATGTCTTACGATGGGGCAATTTTGCAAATAAGAAAACTAAGGAATCAACCCTTCCAGTGCAGTAC CCCAATAGAAATTCAGGAGCAGCTCGTATCTCAACCCTCACCCAACTTCAACCACCTGAAACTTTCAT CCCCAGGTGGGGCTTTGAACCCGGCTTTGAGAACCAAGACTTTAATTATACCCCAACAGATTCCACCA GTGCTACAGGAAAACATTGGACTATTCCGCGGGGCGGGGGAGGACTTTTAGAGTTAGTTTTTAGGCAG ACTTCA GA GACCACTACCTGTGGAGCCTTTTGCTACAAATTAG TTCTTT TAACTAAGGCATCCCGGGC AAGGGTCTTCCACGCGCATGCCTGTTTCAGAGGCTGCCCAGGCCACGTGCACGGA GACCCGGGGCCGT GACCAGGGTGAGACCCGGCAGCCCCACCCACGAACCTGCCCGGTGGCCGCCGCTCCAGCACGTGGGAG TAGCATTTCGGCGACTCCGCTCCGTGCAGCGCGGGGCACTTGCACCTTCCCTCCGCGCTCGCCTGCTG CAGCACCGGACTCCAAGGAGTCCTGAGCTAGGGCTCGCGGAAGGAGAGAGGTCCTGGGTCCCGTCCGC TCTGGGTCCCGCGGAGCCGCGGCCATCTTGGCGGGCTCACCTCGCGGGTCGTGCGCTCGGGCTGGGAC A GGGTGGGGCCCCGGGACCCCACCCCGCTGGGGATCGGGGGGGGGGGGACACGCACCGGAGGA GTGCT CCGTGT GCCGTCACGTGCGCTCCAAGGAGGAGAGTGATGGATGGGGCGACCGCCTGTGCGCGGACTTG GGAGTCCCCACGCGTGGGGGACAGTGGAGCTGAGCCTCCAGTGCAGCTCGA GCCGCTGCGTGACCCTC CGTCCCGGGGTGGGGCACCCTTCCCGAAGCCAAGGCCGAGCGCGGGCTGCGCGGACGCGCGCCCCCCT TCCGGCCGATCCCAGTTTGTGAAGAAAA GGGGCAAAAA GAA GTGTTAAAAGATCCCGGAATTGCGGTT TGGCTC TGGGGCAACGGTTGCTACACGAAGTGAAACCTGCGGAGC GCTCGGGCGCGCGCGCCGCGCCC CGCCCCGATCGGGCGT GACTCATCTTGGGACCGCCCGGCCGGTTCTTATACTCGGGACTGCGGAGCGC GCGCAGGGCTTCCCTCCGCCTGCAGGTCCCGACGCGTCTCCGCCGTCGCCGTCGCCGTCGCCTCCGCC GTACAGCCGAATAGCCGAGGACCCACGCAGAGCCACCAACCAGCCAAGATGCCCAAGCCAGTAAGTTG TCCCTGTTTCGGAGCGGCTGTGCGCCAGGCCGGGTGTCCCCAGGGAATCACCTTGCACTGGCTGCTGG GCAGC TTCTGTA GAACCCAGTT TTCTGGT GAAGGGCCTCTTGTTACCTGTAGACGCCTTTGTGGCACT CTCTAGTCCCCTTTGTGGGTGGTTCTTTTTCGGTTGCTAACGAACCCCCTACCAGGTTTCCGGACTGC TTTAAAAGTGCTTAGTGCAACCTCCAAGTTTCTTAACTGGTGCAAGTAGCACTGAAGCTTTGGTGGCT TCTCACACGACCGACACCTGTTCGCCCAGTTCCTTCGAGCGACCGAA

Exon 1, IFNY-activated site motif, non-optimal IF $N$-activated site motif

Predicted TSS using BDGP neural network prediction tool

\subsubsection{STAT1 readily binds to the tandem GAS motif as a dimer but poorly as a tetramer}

To evaluate whether the identified sequence was in fact an IFN $\gamma$-activated site, gel shift assays were applied using a radioactively labelled probe that contained the native sequence with the tandem GAS motif from the human EZR promoter region. Protein extracts from IFN $\gamma$-treated U3A cells expressing wildtype human STAT1 were incubated with this probe and tested for specific DNA-binding activity (Figure 11A). The autoradiograms showed a specific signal that was interpreted to represent binding of STAT1 to this probe as a dimer, 
while tetrameric complexes were not detectable. The identity of the band was determined through a supershift reaction using an antibody recognizing STAT1 and, as a negative control, STAT3 (Figure 11A, lane 1 and 2).

Whether STAT1 preferentially bound to the first or the second GAS motif of the tandem sequence was then tested. In this study, four different mutant versions of the probe that were termed EZR M1 to EZR M4 were designed. The annealed oligonucleotide termed EZR M1 had an altered first GAS motif and would, therefore, not support STAT1 binding at that site, while for EZR M2 the second GAS motif was altered in the same way. The probe EZR M3 incorporated the mutated sites of both EZR M1 and EZR M2 and was expected not to bind STAT1, and in EZR M4, the GAA of the first and the TTC of the second motif were altered and, therefore, presented with two half-sites. As demonstrated in Figure 11A, lanes 4 to 7, it appeared that there was no preference towards the first or second motif of the tandem GAS element shown by similar signal intensity of the dimer band in lane 4 and 5 . The complex formed was specific since no binding was detected for EZR M3 mutated at both sites (lane 6). EZR M4, however, still showed some, though reduced binding of STAT1 showing that either an intact half site could support the dimer binding or that the dimer could span a longer distance on the DNA with reduced binding affinity.

The tandem GAS element found in the EZR promoter region was different from previously reported tandem GAS motifs. For example, the gene CCL2 was reported to have a one-and-ahalf GAS motif that consisted of a full-length GAS motif and another half-site located at $10 \mathrm{bp}$ distance from the GAS site. This sequence has been discussed to be better at facilitating tetramer stabilization and contributes to the stronger sensitivity of CCL2 gene transcription in response to different phosphorylation levels of STAT1 (Hüntelmann et al. 2014).

In order to confirm that the GAS element found in the EZR promoter did not support tetramer binding, a probe was designed that spanned a larger sequence surrounding the GAS motif which was termed EZR long. This probe was tested with extracts of U3A cells expressing wildtype STAT1 or a mutant of STAT1 with point mutations in positions 92 and 96 which had been shown to provide significantly stronger stabilization of STAT1 tetramers (Staab et al., 2015). When testing this probe a prominent signal resembling binding as a tetramer was detected for the STAT1-DE92/96RR (Figure 11B, lane 4), while the corresponding signal for the wildtype molecule was hardly detectable (lane 2). In contrast, binding of dimeric STAT1 to the probe ERZ long was found for both STAT1 variants. Thus, it was assumed that the sequence found in the promoter region of the human EZR gene is 
readily occupied by phospho-STAT1 dimers but represents only a weak target for tetramer binding.

A

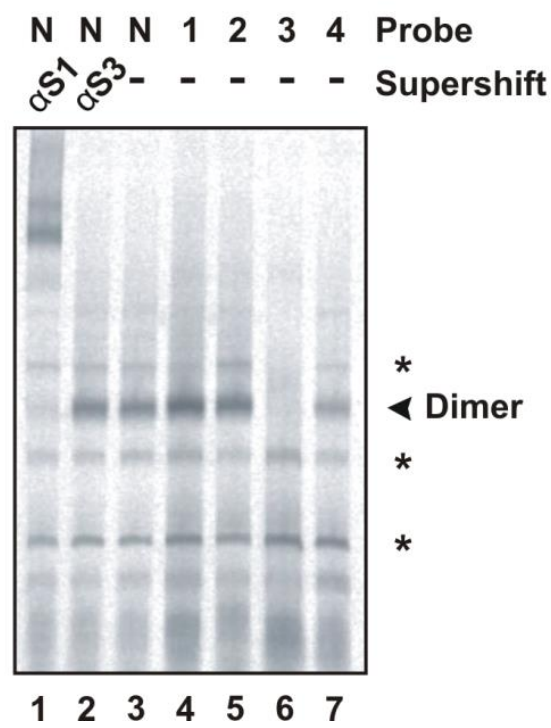

B

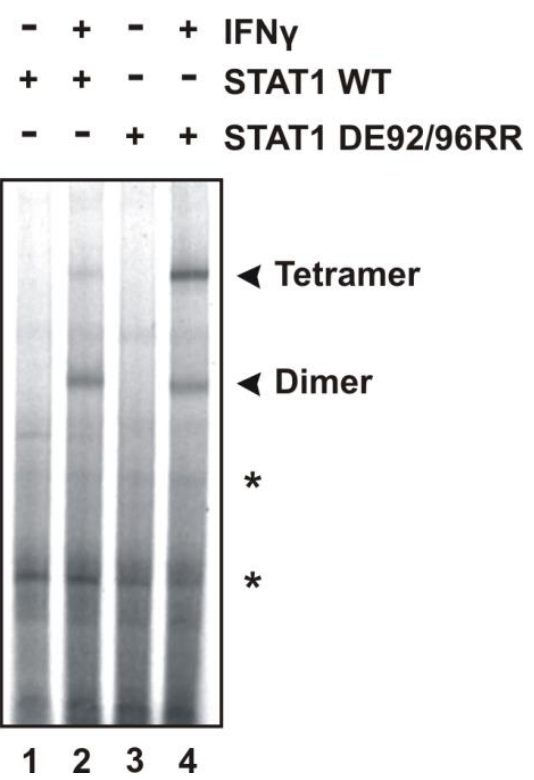

Figure 11: EMSA showing STAT1 binding to tandem GAS elements in the human EZR promoter. (A) Native (N) and mutant versions (probes 1-4) of the tandem GAS motif found in the human EZR promoter were incubated with protein extracts of STAT1-expressing U3A cells treated with IFN $\gamma$ and $\mathrm{Na}_{3} \mathrm{VO}_{4}$. Probes used were native EZR short (N) (5'-TTTTTAATTCCTAGAATTTCAGAGAATC-3'), EZR MI (1) (5'-TTTTTAATAGCTACTATTTCAGAGAATC-3'), EZR M2 (2) (5'-TTTTTAATTCCTA GAATTAGAGACTAATC-3'), EZR M3 (3) (5'-TTTTTAATAGCTACTATTAGAGACTAATC-3'), EZR $M 4$ (4) (5'-TTTTTAATTCCTAGTATTACAGAGAAATC-3'). $\alpha S 1$ = STAT1-specific antibody, $\alpha S 3=$ STAT3-specific antibody. (B) Extracts from U3A cells reconstituted with either wildtype STAT1 or the DE92/96RR double mutant that had been pre-treated with IFN $/ \mathrm{Na}_{3} \mathrm{VO}_{4}$ (lane 2 and 4) or left untreated (lane 1 and 3) were tested with the probe EZR long (5'-TTTTCAAACAAAAAAA CTTAATTCCTAGAATTTCAGAGAAATCCCATGGAGCTTTTT-3'). Asterisks mark unspecific bands.

\subsubsection{STAT1 associates to the EZR promoter in Jurkat cells}

After showing that tyrosine-phosphorylated STAT1 binds to the GAS elements in the promoter of the EZR gene, the next step was to evaluate whether this association was also present in living cells. To this end, we used extracts from Jurkat cells which are a well-studied cell line derived from a T cell leukaemia (Gillis and Watson, 1980). Analysis of STAT1 binding to the EZR promoter using the ChIP technique revealed that, after stimulation of cells with IFN $\gamma$, the respective DNA segment can be precipitated using an antibody targeted to STAT1 while under unstimulated conditions, no precipitation was observed (Figure 12). The 
association of STAT1 to the IRF1 promoter was included as a positive control. This result showed that STAT1 is recruited to the EZR promoter in IFN $\gamma$-treated Jurkat cells.
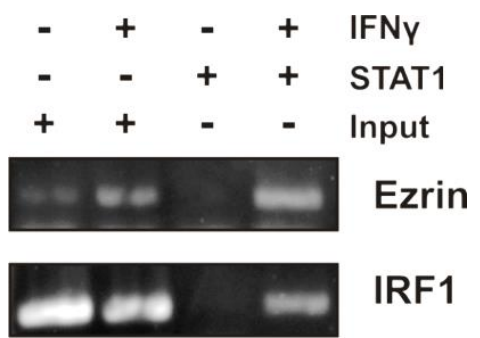

Figure 12: ChIP analysis of STAT1 association to the EZR promoter in IFNy-treated Jurkat cells. Shown is a representative result of a ChIP assay with an anti-STAT1 antibody used for the pulldown including the corresponding input (cross-linked and pre-cleared sample before pulldown). The experiment was performed in Jurkat cells treated with recombinant IFN $\gamma$ for $45 \mathrm{~min}(+)$ and in untreated Jurkat cells (-). For evaluation, the precipitated fragments were amplified using conventional PCR and then visualized by means of agarose gel electrophoresis. IRF1 was analysed as a positive control.

\subsubsection{IFN $\gamma$-activated STAT1 induces $E Z R$ gene transcription}

Since IFN $\gamma$-activated STAT1 is recruited to the promoter of the EZR gene, whether this process had an effect on gene transcription was of interest. As a measure for endogenous gene expression, quantitative PCR (qPCR) was used on reversely transcribed RNA isolates. Jurkat cells were chosen as the suitable system since, in this cell line, the ChIP experiments described above had identified the association of STAT1 to the EZR promoter. HL-60 cells, a myeloid progenitor leukaemia cell line (Collins et al., 1977), were used as a control to confirm the results obtained in Jurkat cells. The data showed that in Jurkat cells, ezr expression increased slightly after $6 \mathrm{~h}$ of incubation with IFN $\gamma$. This trend continued and was even more pronounced after $10 \mathrm{~h}$ incubation and led to a roughly three-fold induction (Figure 13A). Control experiments with known targets of IFN $\gamma$-induced STAT1 signalling, namely irfl and stat1, confirmed that the experimental conditions were suitable to induce STAT1dependent gene transcription but also showed that the induction of the EZR gene was comparably mild (Figure 13B and 13C). Similar results were obtained from HL-60 cells. Expression of the EZR gene was mildly induced by IFN $\gamma$ after $6 \mathrm{~h}$ and $10 \mathrm{~h}$ of incubation (Figure 13D). Control analysis of irfl and stat1 was again in line with the results from Jurkat cells (Figure 13E and 13F). 
A

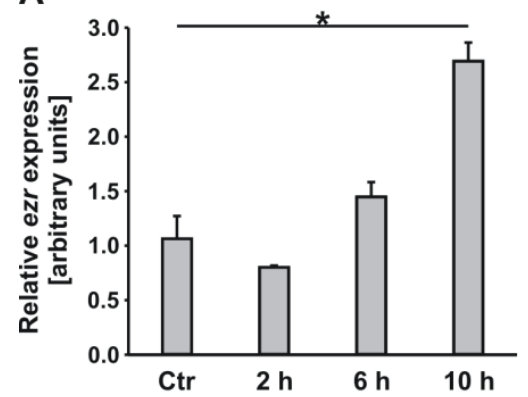

D

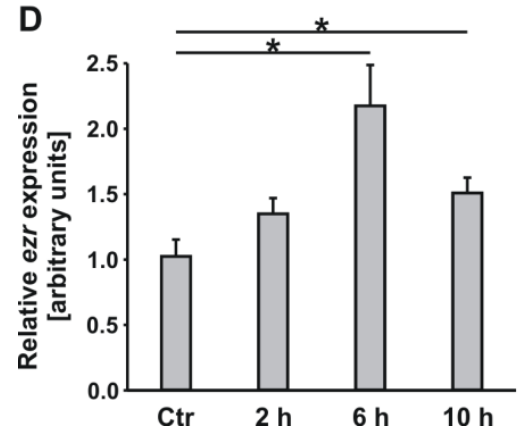

B

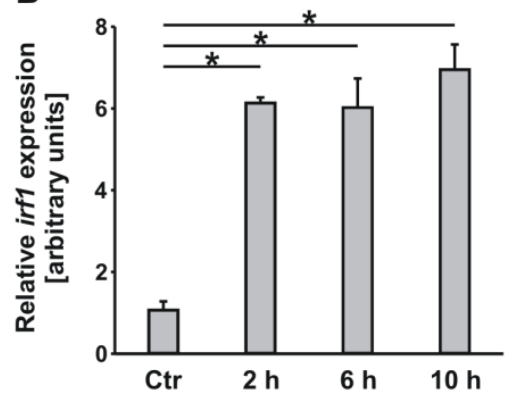

E

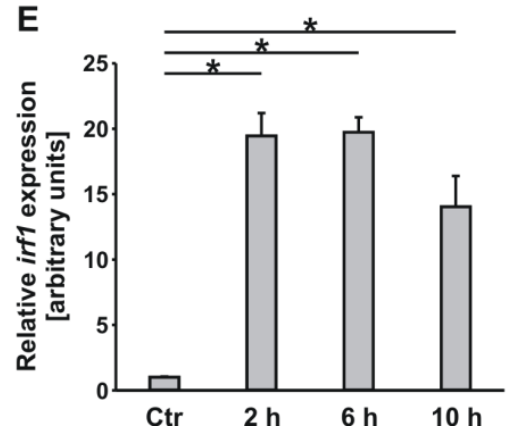

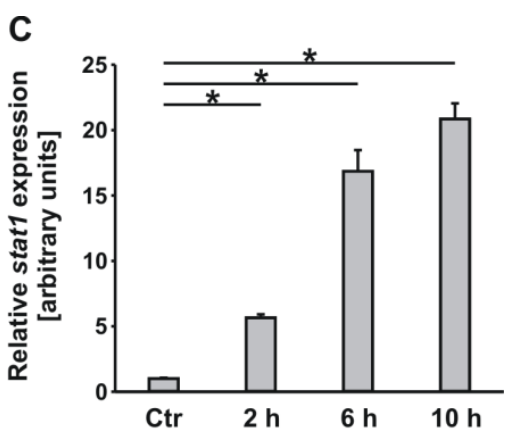

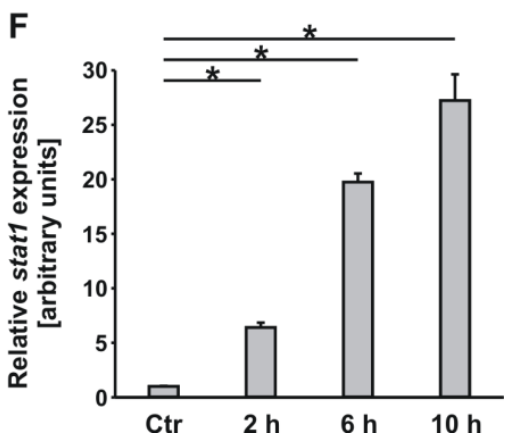

Figure 13: Real-time PCR results from Jurkat cells and HL-60 cells treated with IFN for 2 h, 6 h, and $10 \mathrm{~h}$, respectively. (A-C) Jurkat cells, (D-F) HL-60 cells. Expression of ezrin (A,D), irfl (B,E) and stat1 $(C, F)$. Evaluation was performed using the $\triangle \triangle C T$ method. Shown are representative results. Asterisks indicate statistically significant differences. Error bars represent standard error of the mean.

In order to evaluate whether the observed gene induction was specific for IFN $\gamma$-activated STAT1 signalling, transcription of the EZR gene was analysed also in IFN $\alpha$-treated cells (Figure 14A). It was shown that IFN $\alpha$ was less potent in inducing EZR gene transcription in Jurkat cells as compared to IFN $\gamma$. Analysis of stat1, irfl and ifit1 - an IFN $\alpha$ target gene confirmed adequate stimulation of Jurkat cells (Figure 14B-D).

These data support the hypothesis that EZR is a target gene of IFN $\gamma$-dependent STAT1 signalling. The relatively mild induction of the protein might be attributed to the fact that ezrin is a cytoskeletal protein of which only limited induction is needed. This contrasts to other STAT1-target genes with a much larger increase from baseline expression to cytokineinduced transcription. 
A
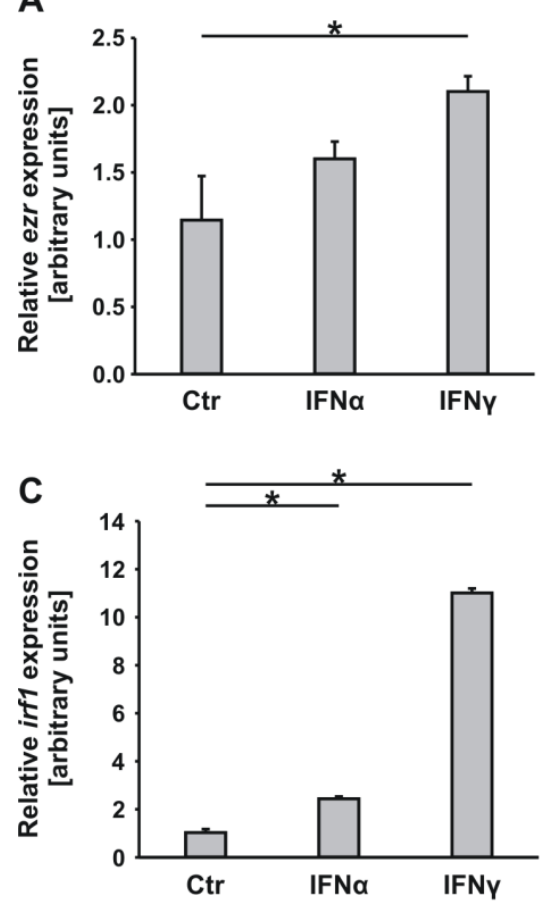

B
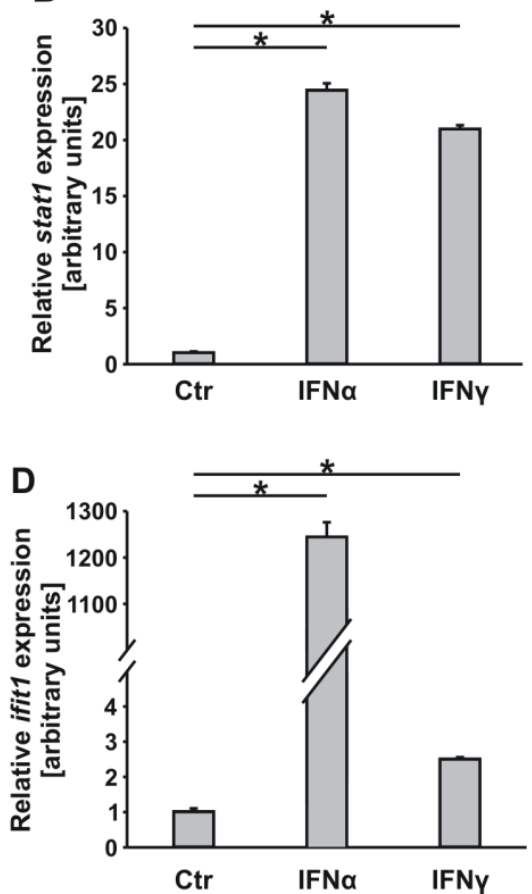

Figure 14: Real-time PCR results of Jurkat cells treated with IFNa and IFNy showing induction of the EZR gene. Expression levels for ezrin (A), stat1 (B), irf1 (C), and ifit1 (D) are shown in unstimulated controls (Ctr) as compared to cells stimulated for $6 \mathrm{~h}$ with either IFNa or IFN $\gamma$. Gene induction was analysed using the $\triangle \triangle C T$ method. Shown are representative results. Asterisks indicate statistically significant differences. Error bars represent standard error of the mean.

\subsubsection{No evidence of IFN $\gamma$-induced ezrin expression in leukaemia cell lines}

Whether stimulation of leukaemia cell lines with IFN $\gamma$ resulted in increased translation of ezrin was then tested. For this purpose, protein lysates were produced using a protocol of fractionated extraction from Jurkat cells and HL-60 cells pre-treated with IFN $\alpha$ or IFN $\gamma$, which were subsequently analysed by means of Western blotting. Stimulation with interferons had no impact on ezrin expression after $4 \mathrm{~h}, 8 \mathrm{~h}$ or $24 \mathrm{~h}$ of treatment, as shown by immunoblotting using an antibody directed against ezrin (Figure 15A and 15E). Quantitation from three experiments confirmed this finding (Figure 15B and 15F). Similar results were obtained using a pan-ERM antibody which detected both ezrin and moesin expression confirming that protein levels of ezrin were unchanged when related to the house-keeping enzyme GAPDH. In addition, no significant differences were seen for moesin expression (Figure 15C, 15D, 15G and 15H). 
A

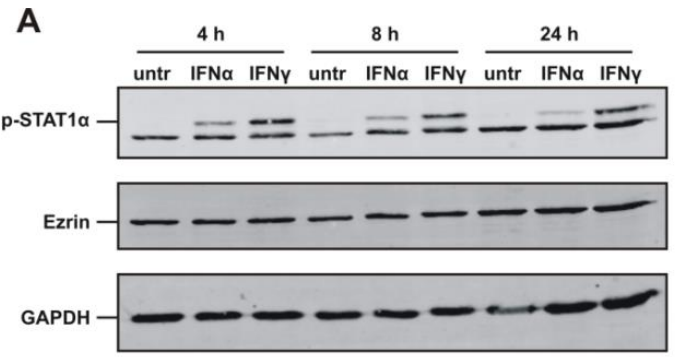

B

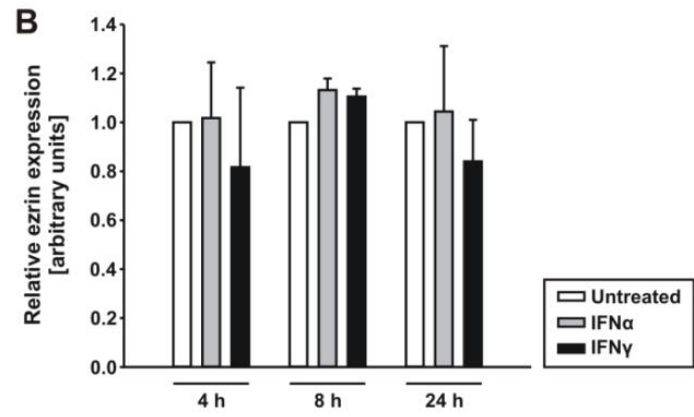

C

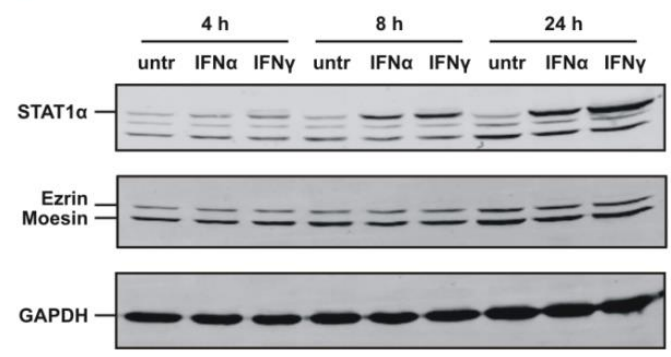

D

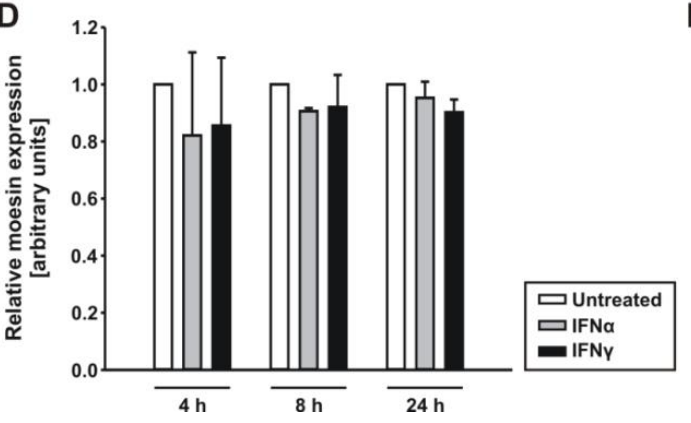

E

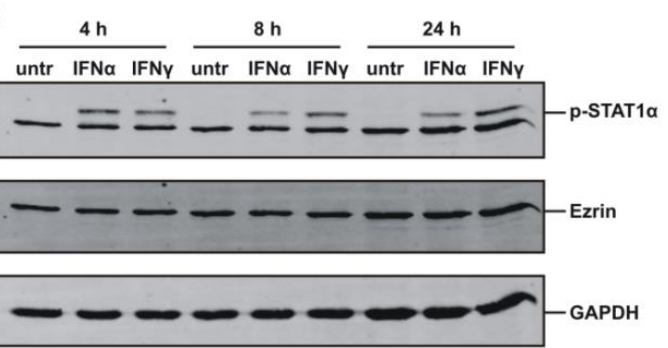

$\mathbf{F}$

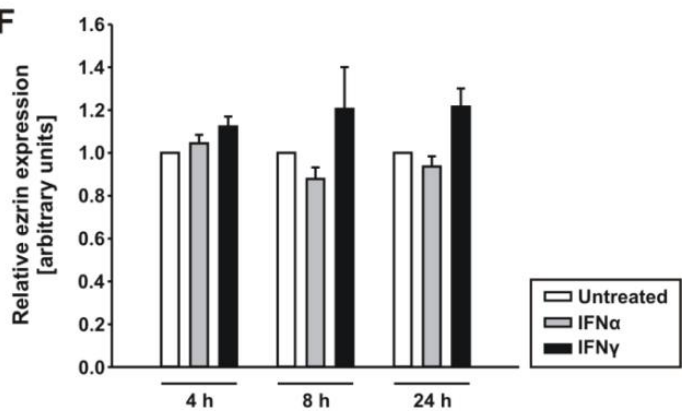

G
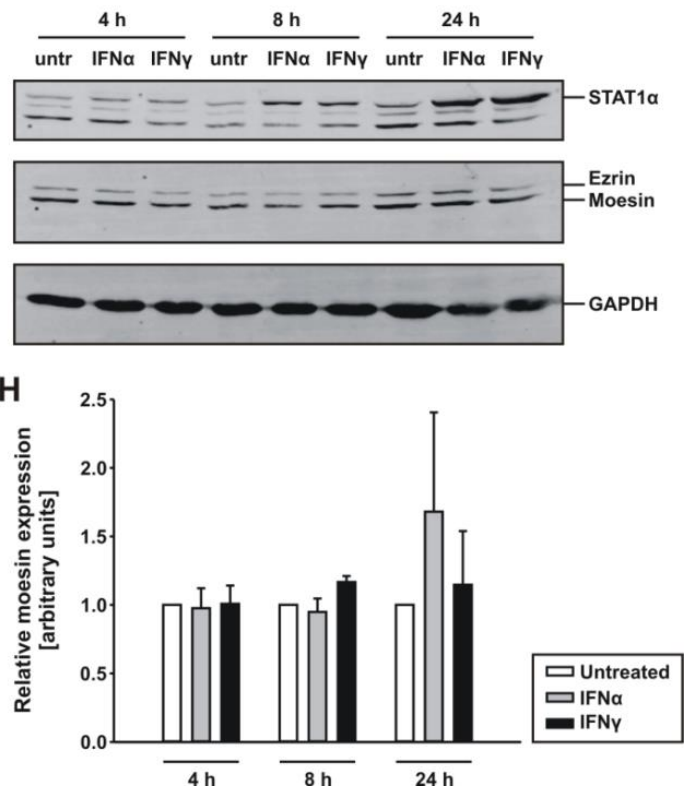

Figure 15: Expression of ezrin and moesin in Jurkat and HL-60 cells treated with IFNa or IFNy. (A) Expression of ezrin in relation to STATl-phosphorylation levels after $4 \mathrm{~h}, 8 \mathrm{~h}$ and $24 \mathrm{~h}$ of IFN $\mathrm{or}$ IFNy treatment in HL-60 cells. (B) Densitometric quantitation of the ezrin signal in (A). (C) Expression of ERM proteins in relation to STATl expression after $4 \mathrm{~h}, 8 \mathrm{~h}$ and $24 \mathrm{~h}$ of IFN $\alpha$ and IFN treatment in HL-60 cells. Ezrin and moesin differ in their molecular weight (approximately by $5 \mathrm{kDa}$ ) and can, therefore, be distinguished, while radixin expression is absent in leukocytes. (D) Densitometric quantitation of the moesin signal in $(C)$. (E) Expression of ezrin in relation to the

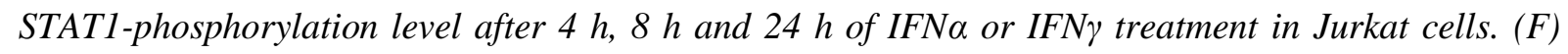
Densitometric quantitation of the ezrin signal in $(E)$. $(G)$ Expression of ERM proteins in relation to STAT1 expression after $4 h, 8 h$ and $24 h$ of IFN $\alpha$ and IFN treatment in Jurkat cells. $(H)$ Densitometric quantitation of the moesin signal in $(G)$. Shown are representative blots. Quantifications show the means of three independent experiments. Error bars represent standard error of the mean. All membranes were first exposed to an antibody against the respective ERM protein, and, subsequently, the same membrane was additionally developed for STAT immunoreactivity. 
A

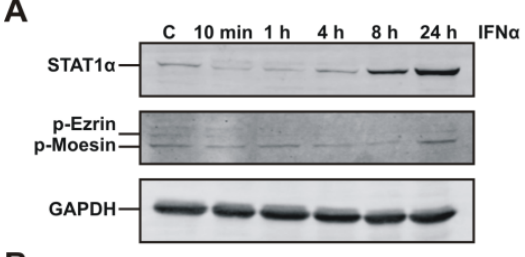

B

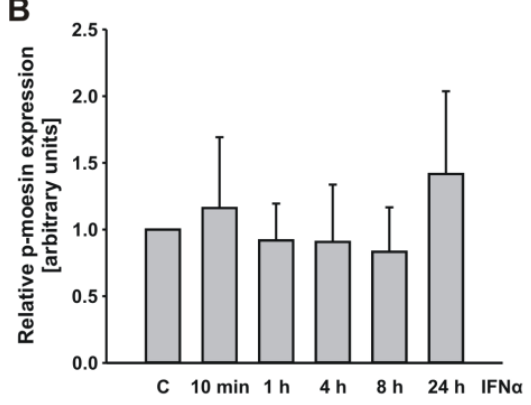

C

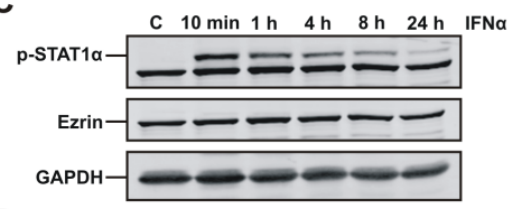

D

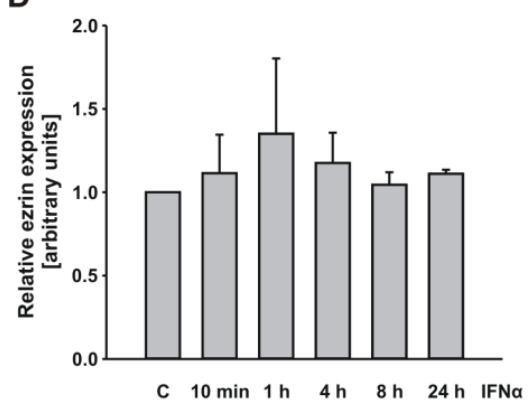

E

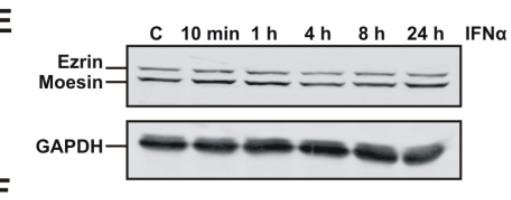

$\mathbf{F}$

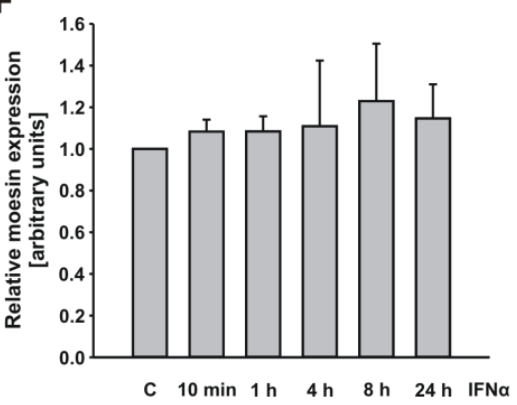

G

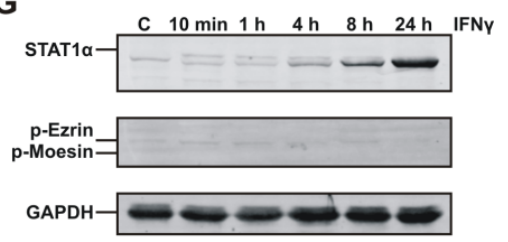

H

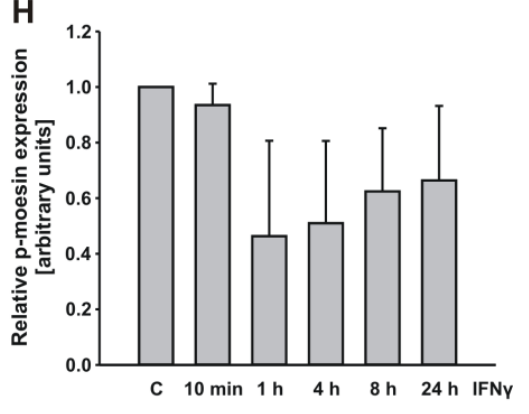

I

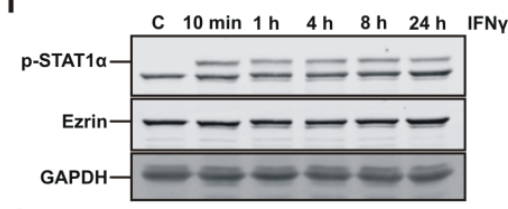

J
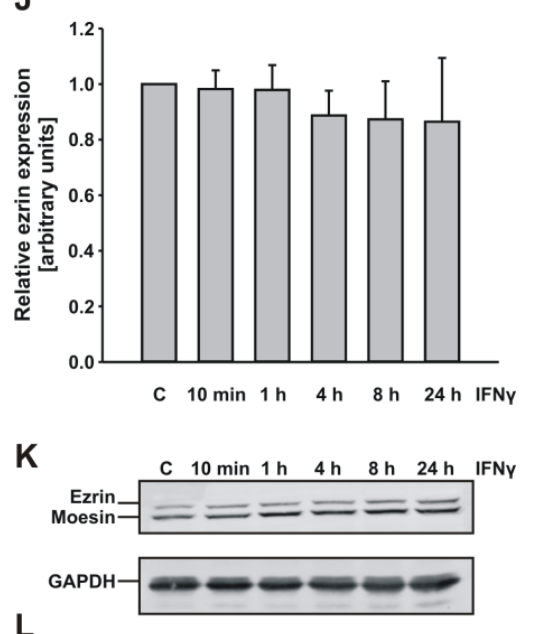

L

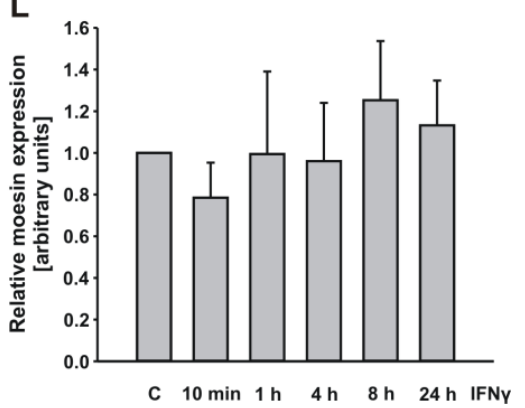

Figure 16: Expression and phosphorylation of ezrin and moesin in HL-60 cells treated with IFNa or IFN $\mathrm{\gamma}$. (A) Phosphorylation levels of ezrin and moesin in relation to STAT1 expression after 10 min, $1 \mathrm{~h}, 4 \mathrm{~h}, 8 \mathrm{~h}$ and $24 \mathrm{~h}$ of treatment with IFN $\mathrm{as}$ compared with the untreated control. (B) Densitometric quantitation of the signal for phosphorylated moesin in (A). Due to low signal intensities, levels of phospho-ezrin were not quantified. (C) Expression of ezrin in relation to phosphorylated STAT1 after indicated times of treatment with IFN . (D) Densitometric quantitation of the ezrin signal in $(C)$. (E) Expression of ERM proteins after the indicated times of treatment with IFNa. $(F)$ Densitometric quantitation of the moesin signal in $(E)$. $(G)$ Phosphorylation levels of ezrin and moesin in relation to STAT1 expression after $10 \mathrm{~min}, 1 \mathrm{~h}, 4 \mathrm{~h}, 8 \mathrm{~h}$ and $24 \mathrm{~h}$ of treatment with IFN $\gamma$ 
as compared with the untreated control. (H) Densitometric quantitation of the signal for phosphorylated moesin in (G). (I) Expression of ezrin in relation to phosphorylated STAT1 in IFN $\gamma$ treated cells, as described above. (J) Densitometric quantitation of the ezrin signal in $(I) .(K)$ Expression of ERM proteins in cells treated with IFN $\gamma .(L)$ Densitometric quantitation of the moesin signal in $(K)$. Shown are representative blots. Quantitations show means of three independent experiments, and error bars represent standard error of the mean. The membranes in $(A),(C),(G)$ and (I) were first exposed to an antibody against the respective ERM protein, and, subsequently, the same membrane was additionally developed for STAT immunoreactivity.

To test whether interferon signalling might have an effect on the phosphorylation levels of ezrin and/or moesin, an antibody targeted to all ERM proteins in their phosphorylated state was used. The respective Western blotting data (Figure 16A and 16G) including their densitometric analysis (Figure 16B and 16H) did not reveal significant differences in the phosphorylation level of moesin for any of the tested treatment durations ranging from $10 \mathrm{~min}$ to $24 \mathrm{~h}$. Ezrin phosphorylation levels were barely detectable and, therefore, not quantified. The data obtained for ezrin and moesin (Figure 16C-F and 16I-L) confirmed the results demonstrated in Figure 15. In summary, the data showed that, despite being weakly activated on the transcriptional level in response to IFN $\gamma$ signalling, ezrin production was not increased at the protein level in two widely used leukocyte-like cell lines.

\subsubsection{Ex vivo analysis of STAT1-deficient and STAT1-F77A mutant mice does not show decreased ezrin protein expression}

Since no ezrin induction on the protein level had been observed in IFN $\gamma$-treated cells, whether baseline ezrin expression was dependent on STAT1 signalling was tested. For this evaluation, bone marrows extracted from mice carrying a deletion of major parts of the STAT1 gene (referred to as STAT1-KO) and mice carrying a point mutation at position 77 which strongly impairs IFN $\gamma$ signal transduction (referred to as STAT1-F77A) were compared to those of wildtype mice.

Figure 17A and 17C shows the baseline expression of ezrin and moesin in bone marrow of three individual animals of each genotype. The blot as well as the corresponding quantitation of ezrin and moesin expression (Figure 17B and 17D) showed no differences between the three genotypes.

To gain a deeper insight into the regulation of ezrin and moesin in murine bone marrow, the isolated cells were incubated with IFN $\alpha$ or IFN $\gamma$ for $45 \mathrm{~min}$ and the whole cell proteins were isolated and analysed by means of Western blotting technique. Again, no significant differences between the three genotypes were detected for the expression of moesin (Figure 
$18 \mathrm{~A}$ and 18B). Analysis of the phosphorylation levels of ezrin and moesin did not reveal a marked difference between the genotypes, neither did the treatment conditions (Figure 18C and 18D). Figure $18 \mathrm{E}$ and $\mathrm{F}$ show the expression and quantitation of ezrin protein expression after stimulation again detecting no differences among the genotypes.
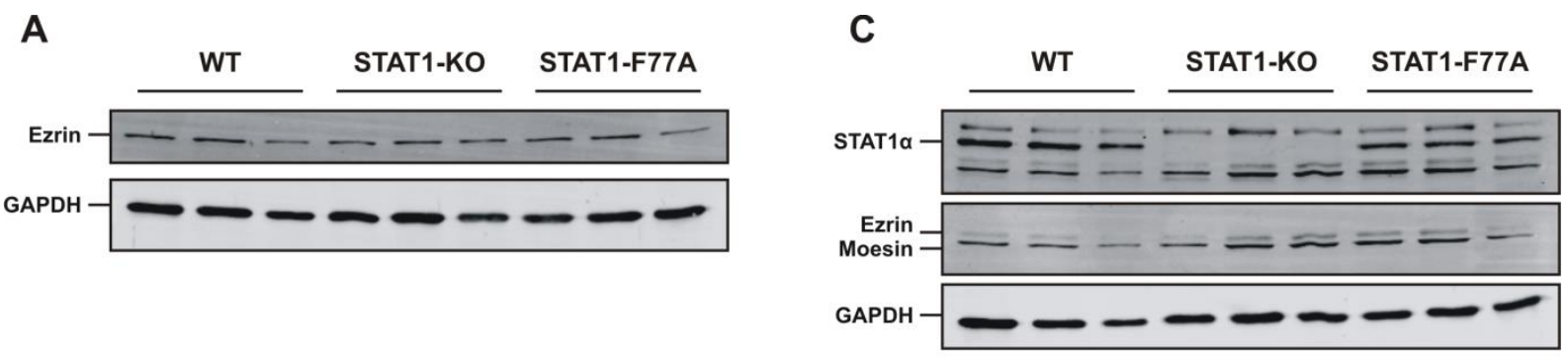

B

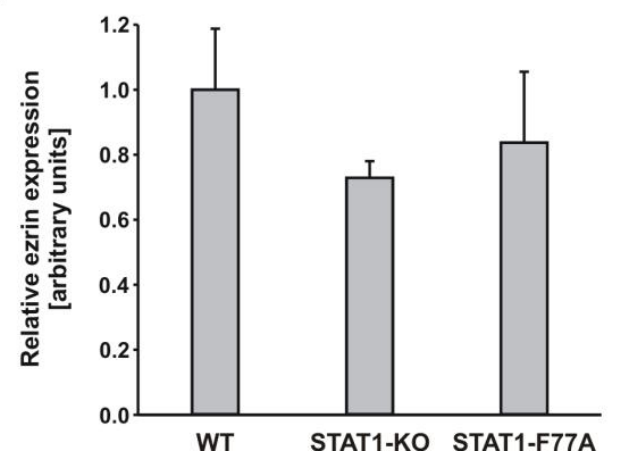

D

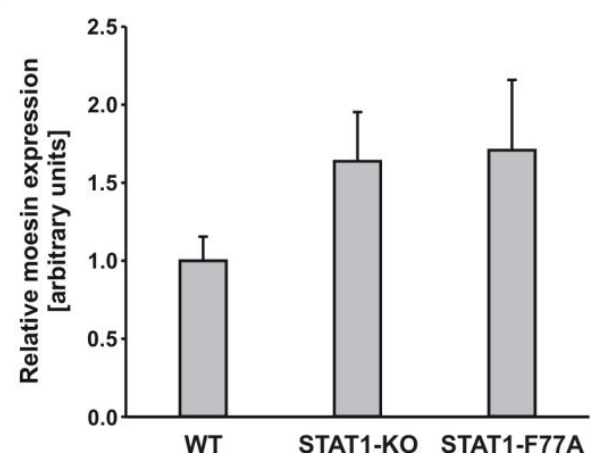

Figure 17: Ex vivo analysis of baseline expression of ezrin and moesin in bone marrow cells from wildtype, STAT1-deficient and STAT1-F77A mice. (A) Expression of ezrin at baseline in protein extracts derived from bone marrow obtained from three individual mice of each genotype (B) Densitometric quantitation of the moesin signal in (A). (C) Expression of the ERM proteins in relation to STAT1 in protein extracts derived from murine bone marrow obtained from three individual animals of each genotype. (D) Densitometric quantitation of the moesin signal in (C). Error bars represent standard error of the mean. In (C), first, immunoreactivity for ERM protein was detected, and in a second step the same membrane was developed for STAT immunoreactivity.

To test the hypothesis that in the absence of STAT1-dependent signalling, STAT3dependent signalling becomes upregulated, STAT3 and p-STAT3 were detected in the bone marrow protein extracts of all three genotypes. Analysis of the phosphorylation level of STAT3 revealed an induction by IFN $\gamma$ and to a limited extent also by IFN $\alpha$. The induction, as well as, the baseline phosphorylation appeared to be slightly more pronounced in the F77A mutant but this effect remained below the level of significance (Figure 18G and 18H). 
A

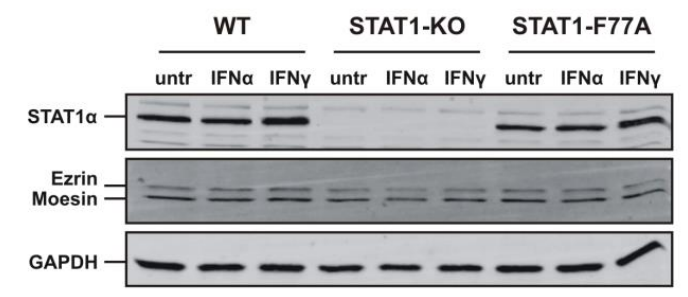

C

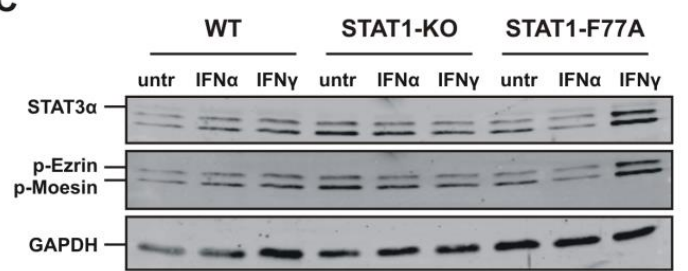

E

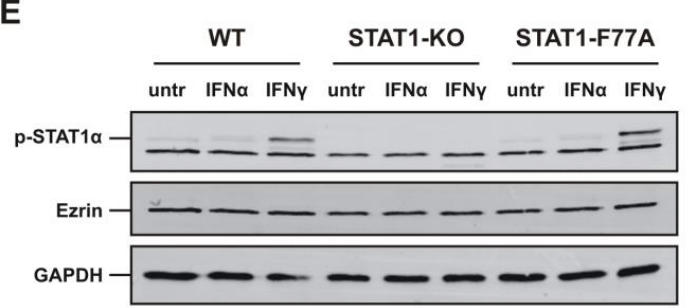

G

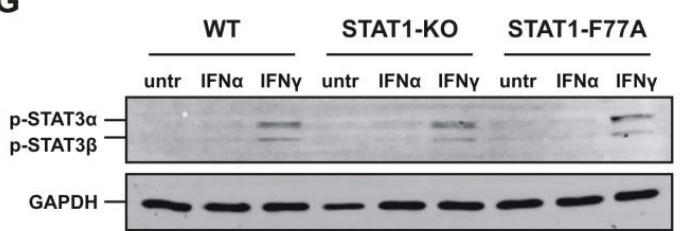

B
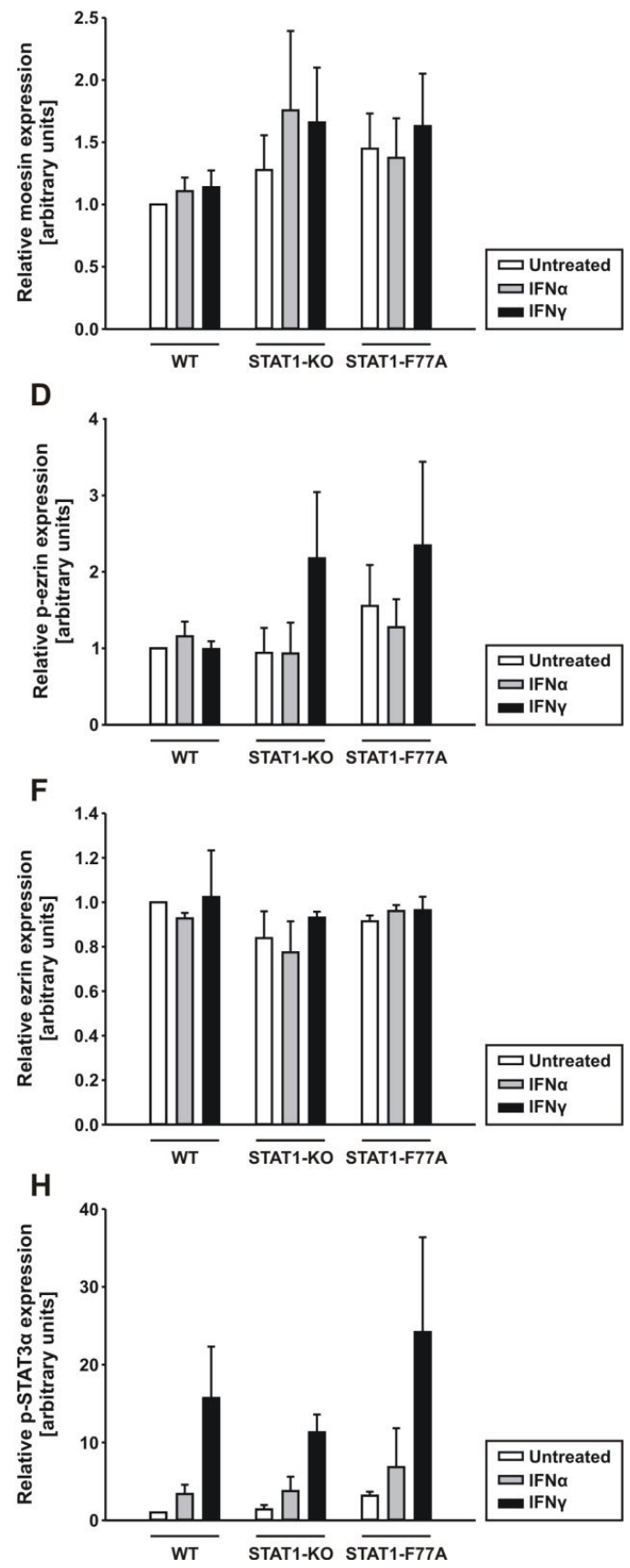

Figure 18: Phosphorylation levels of ezrin and moesin in bone marrow from wildtype, STAT1deficient and STAT1-F77A knockin mice treated ex vivo with interferons. (A) Expression of the ERM proteins in bone marrow cells treated for 0 min (untr) or 45 min with IFNa or IFN $\gamma .(B)$ Densitometric quantitation of the moesin signal in (A). (C) Phosphorylation levels of ezrin and moesin

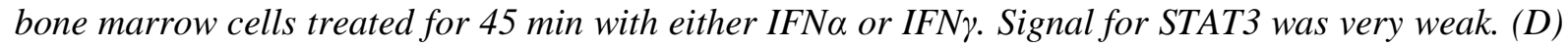
Densitometric quantitation of the phospho-ezrin signal in $(C)$. (E) Expression of ezrin in bone marrow

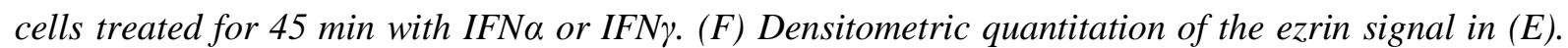
(G) Phosphorylation levels of STAT3 in bone marrow cells treated for 45 min with IFN or IFN $\gamma .(H)$ Densitometric quantitation of phospho-STAT3. Shown are representative blots. Quantitation shows means of three independent experiments. Error bars represent standard error of the mean. First, immunoreactivity for ERM protein was detected in $(A),(C)$ and $(E)$ and in a second step the same membrane was developed for STAT immunoreactivity. 
The data obtained from the two mouse models of impaired IFN $\gamma / \mathrm{STAT} 1$ signalling were in line with the results from Jurkat cells and HL-60 cells which did not show a significant change in ezrin expression upon cytokine stimulation. A mild but consistent and robust induction of EZR gene transcription by IFN $\gamma$ signalling in both cell lines tested was shown. However, an increased production of ezrin at the protein level was neither detected in human cell lines nor in murine bone marrow cells.

\subsection{Assessment of the role of IFN $\gamma$-dependent STAT1 signalling in myocardial infarction}

Upon myocardial infarction, functional cardiac tissue is not only lost due to ischemia-related tissue necrosis but also by an accompanying inflammatory reaction (Bonaventura et al., 2016). Inhibiting the recruitment of inflammatory cells to the myocardium is hypothesized to be a promising approach to favour repair and reduce injury. With respect to the role of STAT1 in the context of cardiac ischemia, it was shown that STAT1 signalling favours apoptosis over autophagy, as demonstrated in an ischemia/reperfusion model using the Langendorff heart (McCormick et al., 2012). Since this model ignores the role of the immune reaction, the focus of this project was set on an in vivo model of myocardial infarction where cardiac function as well as the immune response can be monitored.

\subsubsection{General characteristics of the study cohort and infarct survival}

Mice carrying the STAT1-F77A mutation on a C57B1/6N background as well as wildtype control mice were performed surgery on to induce myocardial infarction by ligating the left anterior descending artery. Table 25 summarizes the general characteristics of the study cohort. Mice were divided into a group that was sacrificed three days after surgery to analyse the hearts during the acute phase of inflammation and a second cohort that was kept for 4 weeks after surgery to acquire data on survival and cardiac remodelling. Echocardiography was performed before and 3 days, 1 week and 4 weeks after surgery to monitor cardiac function and remodelling. Additionally, samples were taken on day 3 for histological and biochemistry analyses to evaluate cardiac inflammation during the acute phase of myocardial infarction. Heterozygous mice were only included for the early analysis. 
Table 25: Survival rates of mice after undergoing myocardial infarction surgery at day 3 postmyocardial infarction

\begin{tabular}{|c|c|c|c|c|c|c|}
\hline \multirow{2}{*}{} & \multicolumn{3}{|c|}{ MI/Sham } & \multicolumn{2}{c|}{ MI Survival/Death } \\
\cline { 2 - 7 } & Total & + & $\sigma^{\lambda}$ & Total & + & $\sigma^{\lambda}$ \\
\hline Total & $125 / 23$ & $59 / 11$ & $66 / 12$ & $117 / 8$ & $56 / 3$ & $61 / 5$ \\
\hline WT & $50 / 8$ & $23 / 4$ & $27 / 4$ & $47 / 3$ & $22 / 1$ & $25 / 2$ \\
\hline WT/F77A & $28 / 7$ & $14 / 3$ & $14 / 4$ & $25 / 3$ & $13 / 1$ & $12 / 2$ \\
\hline F77A/F77A & $47 / 8$ & $22 / 4$ & $25 / 4$ & $45 / 2$ & $21 / 1$ & $24 / 1$ \\
\hline
\end{tabular}

All animals which survived the MI-surgery were observed for at least three days before being sacrificed in order to collect cardiac tissue for further analysis. Within the first $48 \mathrm{~h}$ after myocardial infarction, the mice lost a mean $9 \%$ of their body weight but started to gain weight again and were only $5.2 \%$ below their pre-surgery body weight on day 3 . This observation was similar in wildtype, heterozygous and STAT1-F77A knockin mice and did not differ significantly between males and females. The overall survival rate of the surgery during the first three days was $88 \%$ without significant differences among the genotypes. Females displayed a slightly higher survival rate $(92.5 \%)$ than males $(84.8 \%)$ which was in line with studies using different models of myocardial infarction, heart failure and atherosclerosis which describe beneficial effects of oestrogen (Piro et al. 2010).

In the cohort for the 4 week follow-up, only homozygous animals were included. The higher mortality of males as compared to females was confirmed. Data on the weight loss after surgery confirmed the observation that after an initial weight loss of about $10 \%$ of the original body weight within the first day after surgery, mice started gaining weight again until reaching a weight gain of roughly $7 \%$ of the pre-surgery weight at 4 weeks post-infarction. No significant differences between the genotypes were observed with respect to changes in body weight.

During the chronic follow-up, a high number of animals (in total $40 \%$ of the study cohort) died or had to be euthanized. The mortality was non-significantly higher in the wildtype as compared with the STAT1-F77A knockin cohort (47.8 \% 4-week-survival in the wildtype group as compared to $72.7 \%$ 4-week-survival in the knockin group). The analysis of these data for males and females separately, showed that the effect disappeared for female animals while becoming significant in males for the 1 -week as well as the 4 -week time point. Figure 
19 presents the Kaplan-Meier survival curves of the animals included in the study cohort up to 4-weeks after myocardial infarction. The data showed that while survival rates in females are in general high, a large proportion of male mice died between the $4^{\text {th }}$ and $7^{\text {th }}$ day after myocardial infarction. This drop in survival was significantly more pronounced in wildtype animals than in the STAT1-F77A knockin mice. Therefore, loss of the IFN $\gamma$-STAT1 axis was considered to be beneficial in male mice undergoing myocardial-infarction surgery.

A

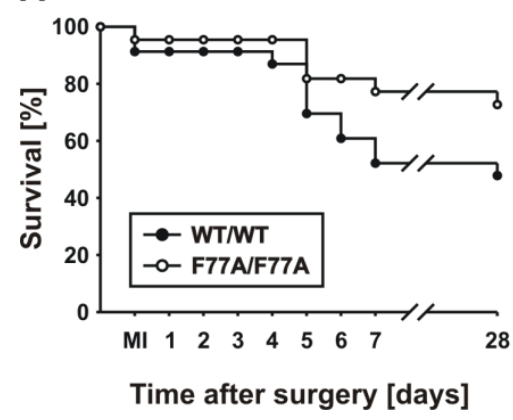

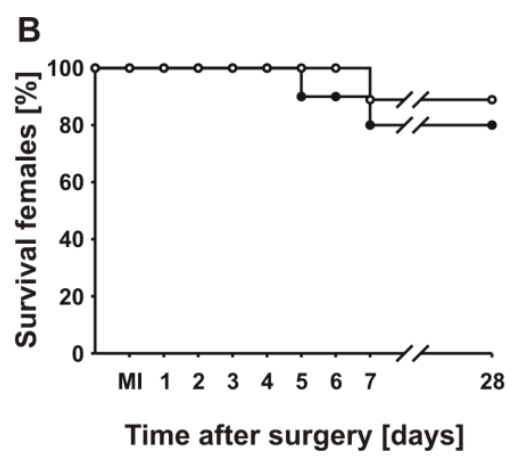

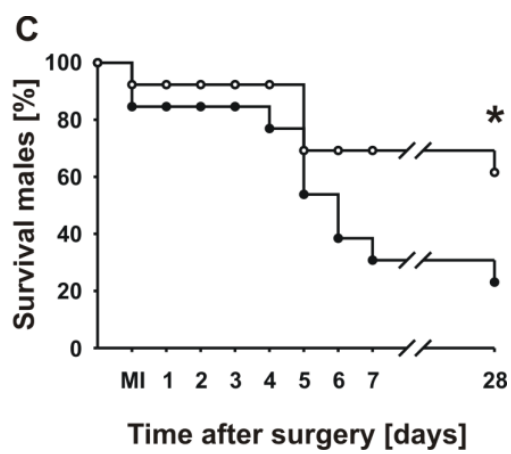

Figure 19: Kaplan-Meier survival analysis of mice subjected to experimental myocardial infarction. Shown are the survival rates for all animals in the study group included in the 4-week analysis. Animals of both sexes $(A)$ as well as exclusively female $(B)$ and male $(C)$ mice were evaluated. Asterisk indicates statistically a significant difference.

\subsubsection{H\&E staining did not reveal differences in the size of the infarcted area}

In order to assess the extent of inflammation of the ventricular wall during myocardial infarction, mice were sacrificed 3 days after MI surgery, and their explanted hearts were immediately fixed and embedded in paraffin. To allow for an estimation of the position of the sections used for staining, sections of $5 \mu \mathrm{m}$ thickness were produced in a serial manner. Sections taken roughly $1.2 \mathrm{~mm}, 1.6 \mathrm{~mm}, 2.0 \mathrm{~mm}$ and $2.4 \mathrm{~mm}$ from the apex of the heart, corresponding to the entire region of the infarcted area, were subjected to H\&E staining. Figure 20 shows representative sections of MI and sham-operated hearts of each genotype at $\sim 1.6 \mathrm{~mm}$ from the cardiac apex. All samples except the sham-operated hearts showed pronounced accumulation of invading leukocytes after myocardial infarction, as can be concluded from the presence of numerous cells with a small cytoplasmic rim and haemalumstained nuclei.

In order to quantify the extent of the inflamed area of the cardiac muscle, the angle for distribution of inflammatory foci over the entire circumference of the left ventricle was assessed (Figure 21A). Results showed that the size of the infarcted area did not differ 
between wildtype, STAT1-F77A knockin and heterozygous mice, as judged from the measurement of the circumference affected by infiltrates.

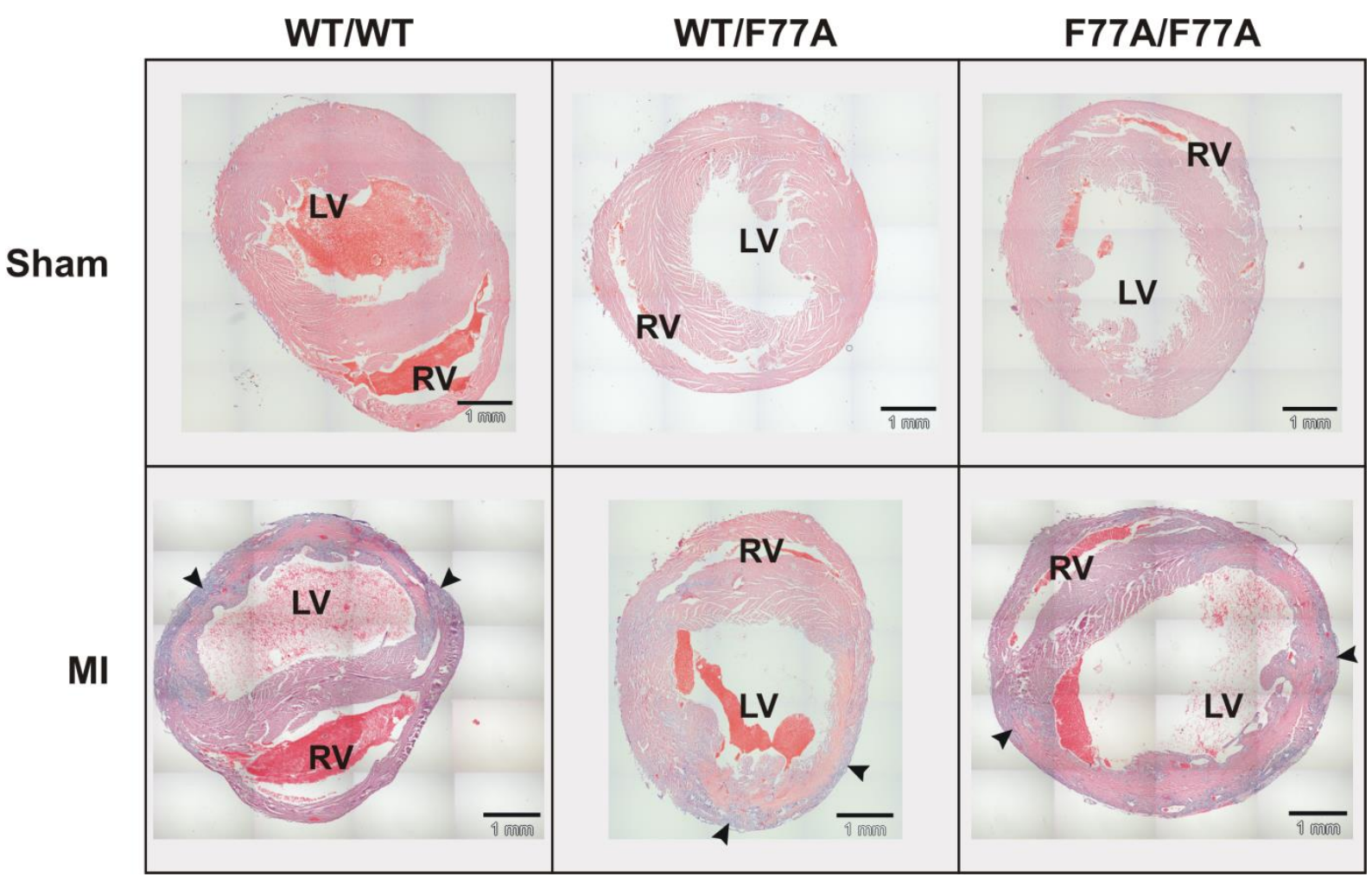

Figure 20: $\boldsymbol{H} \& \mathrm{E}$ staining of acute myocardial infarction. Shown are representative slides of hearts 3 days after MI or sham operation of each genotype. Strong infiltration of the left ventricular myocardium is visible in all three genotypes after infarction but not in the hearts of sham-operated animals. Arrowheads mark representative areas of infiltration. LV: left ventricle, RV: right ventricle. Images are montages of 15 to 20 individual images.

To take into account the severity of the inflammation and not only the distribution, a score between 0 (no inflammation) and 4 (very severe inflammation) was assigned to the $\mathrm{H} \& \mathrm{E}$ stained MI samples at each of the aforementioned positions (Figure 21B). Again, there was no significant difference in the score assigned to the infarcted sections between the different genotypes. Figure $21 \mathrm{C}$ shows the results of the consecutive ranking of the extent of inflammation. There appeared to be a mild tendency of the STAT1-F77A samples towards a score at a slightly higher rank than the wildtype counterparts but the effect was below the level of significance and is not supported by the data of the heterozygous animals which received the lowest mean rank. These data showed that wildtype and STAT1-F77A knockin mice did not differ with respect to the severity and distribution of inflammatory foci in their hearts in the acute phase of inflammation, 3 days after myocardial infarction. 

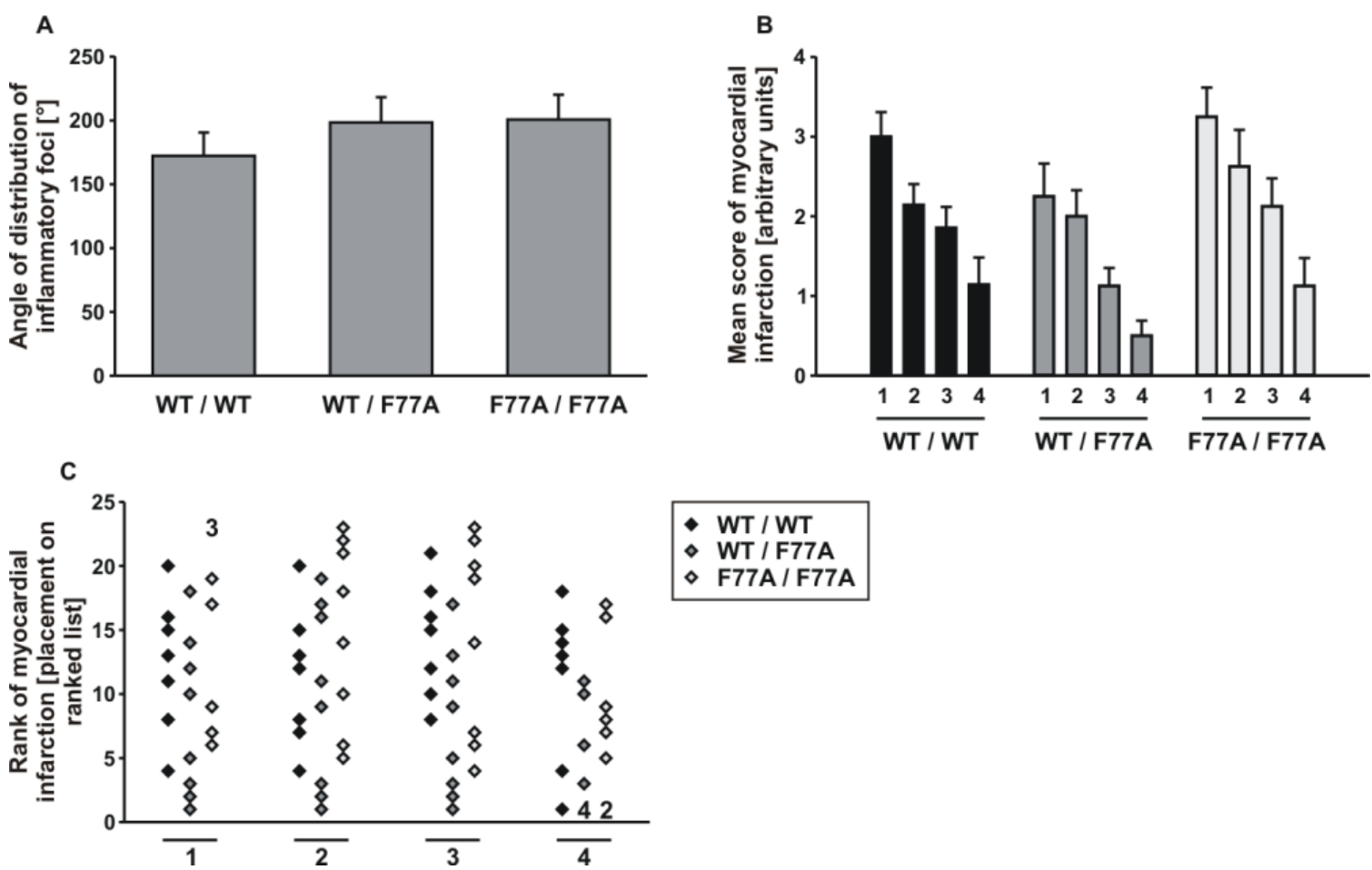

- WT I WT

-WT / F77A

$\diamond$ F77A / F77A

Figure 21: Evaluation of the extent of inflammation according to $H \& E$ staining of sections of paraffin-embedded cardiac tissue three days after myocardial infarction. (A) The distribution of inflammatory infiltrates was assessed by determining the angle from the centre of the left ventricle in which areas of pronounced accumulation of small cells (assumed to be leukocytes) were located. The angle was determined for sections in the middle of the infarcted area. The mean was calculated for each genotype. Error bars represent standard error of the mean. (B) An arbitrary score was assigned to each infarcted heart in serial section near the apex (1, $1.2 \mathrm{~mm}$ from apex), in the middle of the infarcted area (2, 1.6 $\mathrm{mm}$ from the apex), in the upper part of the infarcted area (3, 2 $\mathrm{mm}$ from the apex) and at the top part of the infarcted area (4, $\sim 2.4 \mathrm{~mm}$ from the apex). The score " 0 " was assigned to sections without inflamed areas, "1" for small, restricted areas of inflammation of less than half the left ventricular wall, "2" for distribution of inflammatory foci over up to $90 \%$ of the left ventricular wall, "3" for entire left ventricle with little or no involvement of septum and right ventricle and "4" for involvement of septum and right ventricle. Error bars represent standard error of the mean. (C) Scatter blot of the consecutive ranks assigned to each MI heart. Largest infarction was ranked highest. Each point corresponds to one heart at the respective position. Numbers indicate that several samples were assigned the same rank. For wildtype, 7 cases were analysed, and for heterozygous and knockin 8 animals were included.

\subsubsection{Mice carrying the STAT1-F77A mutant are protected from adverse cardiac remodelling}

In the acute phase after myocardial infarction, immune cells are recruited to ischemic areas of the heart during a first inflammatory phase (reviewed in Bonaventura et al., 2016). This inflammatory reaction begins within the first hours after the insult and is resolved after a few days. It has been noted that the inflammatory environment extends beyond the ischemic tissue and may cause additional damage in the border zone. Shortly after the invasion of the tissue 
by inflammatory cells, anti-inflammatory signalling starts to facilitate clearing of the inflammation. Following survival of myocardial infarction, patients often suffer from heart failure due to post-infarction left ventricular remodelling (reviewed in Galli and Lombardi, 2016). During this process, fibrotic scar formation of the previously hypoxic tissue is accompanied by an alteration in matrix composition as well as the absence of contracting cardiomyocytes in this area. As a consequence, the left ventricle is often dilated resulting in a permanently reduced ejection fraction.

A

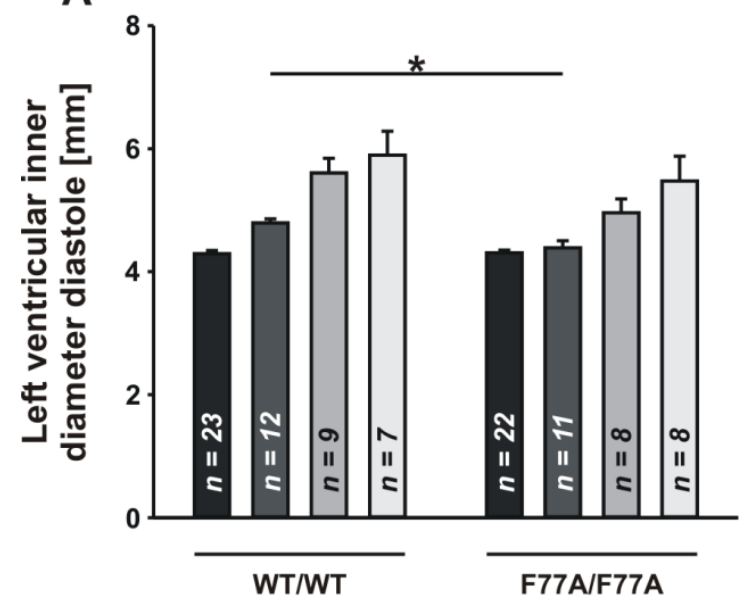

B

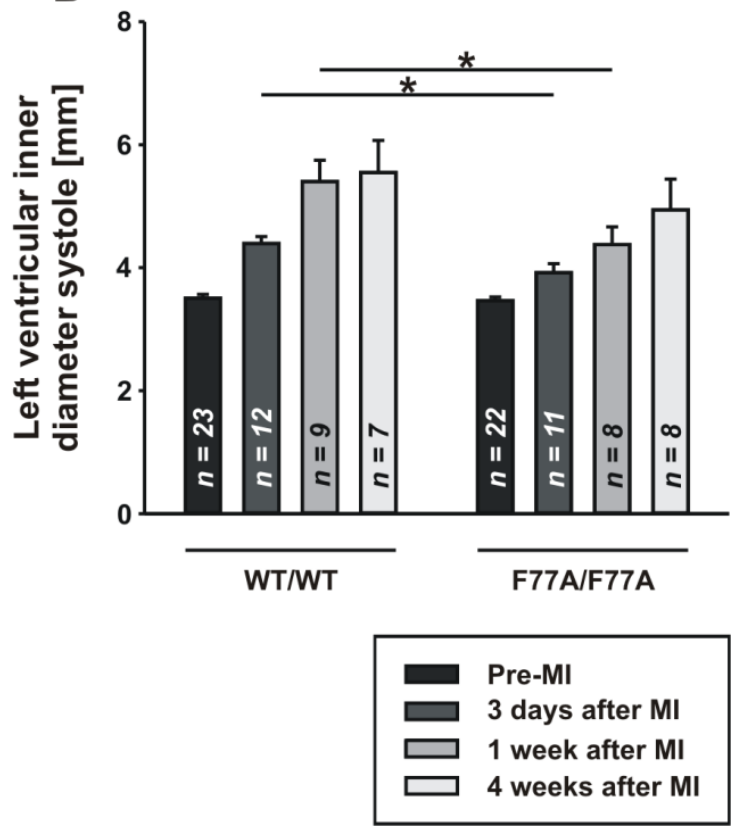

Figure 22: Increase in left ventricular diameter after myocardial infarction. The diameter of the left ventricular lumen was measured in diastole (A) and systole (B) in wildtype and STAT1-F77Aexpressing mice using echocardiographic images obtained in $B$ mode. For all mice analysed, preinfarction data were acquired. Mice were analysed either during the acute phase (analysis on day 3 after infarction) or kept for monitoring cardiac remodelling (analysis 1 week and 4 weeks after infarction). Shown are only the data acquired from female mice. The numbers of mice included in this experiment is indicated inside the columns. Asterisks indicate statistically significant differences. Error bars represent standard error of the mean.

In order to evaluate the adaption of the cardiac tissue in the absence of IFN $\gamma$ signalling, echocardiography was performed on mice, three days, one week and four weeks after ligation of the left anterior descending coronary artery and compared with the measurement obtained before surgery (mice of the day-3 cohort were analysed before and 3 days after surgery, mice of the 4-week cohort were analysed before and 1 week after and 4 weeks after surgery). Based on anatomical measurements of left ventricular dimensions, functional data were computed. 
A
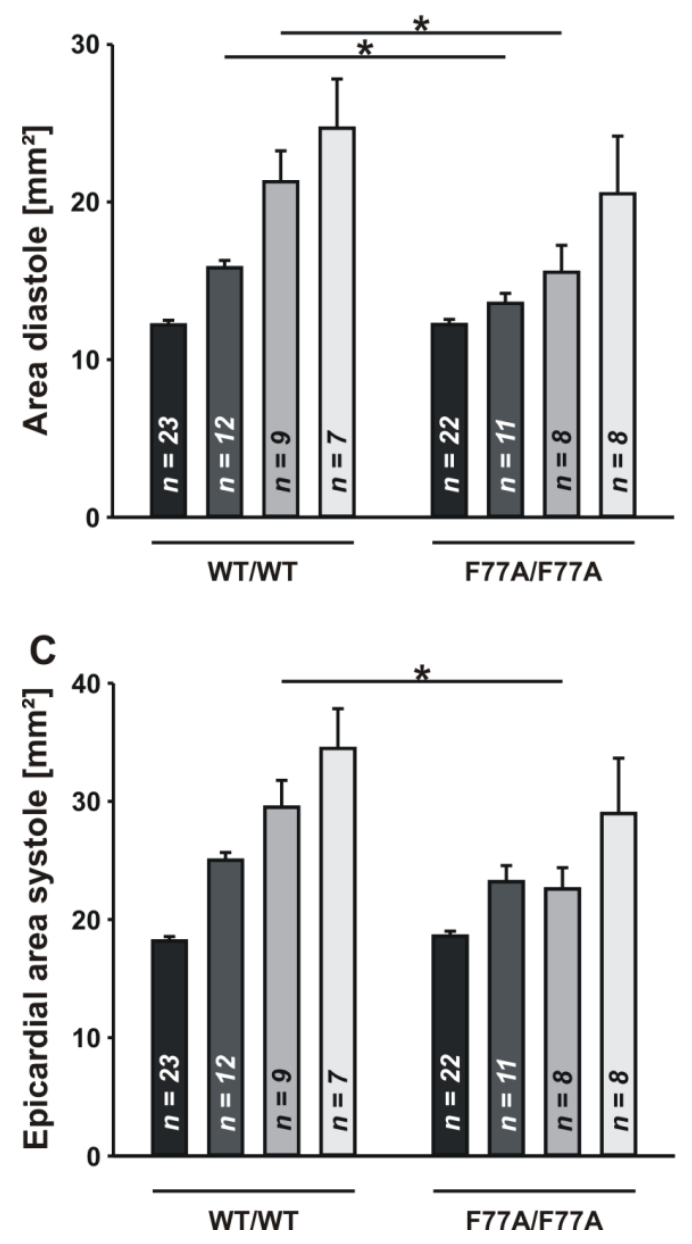

B

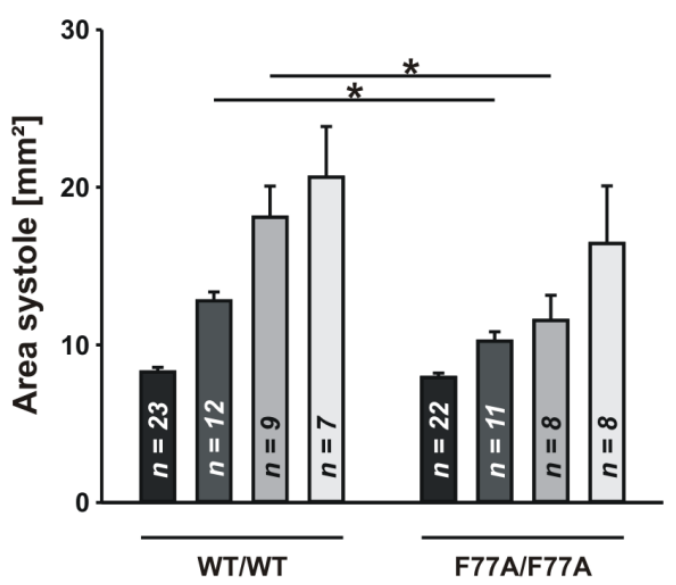

Figure 23: Increased left ventricular cross-sectional area due to myocardial infarction. The crosssectional area of the left ventricular lumen and the epicardial area were measured using echocardiographic images obtained in B mode. (A) Left ventricular cross-sectional area of the lumen in diastole. (B) Measurements obtained in systole. (C) End-systolic epicardial cross-sectional area. For all mice analysed, pre-infarction data were acquired. Mice were analysed either during the acute phase (analysis on day 3 after infarction) or kept for monitoring cardiac remodelling (analysis 1 week and 4 weeks after infarction). Shown are only the data acquired from female animals. The numbers of mice included are indicated inside the columns. Asterisks indicate statistically significant differences. Error bars represent standard error of the mean.

Analysis of the entire cohort showed no differences in cardiac remodelling, shape or performance between the wildtype and the STAT1-F77A knockin mouse. Variance of all measurements was high. Separation of the data obtained from male and female mice and separate analysis of these produced a drop in variance. In females carrying the STAT1-F77A knockin allele, the increase in both diastolic and systolic left ventricular diameter was attenuated during short-term follow-up (Figure 22). Similar differences between female wildtype and knockin littermates were detected for the area of luminal and epicardial cross- 
sections 3 days and 1 week following ligation of the coronary artery (Figure 23). In male mice, no difference was found among the genotypes (data not shown).
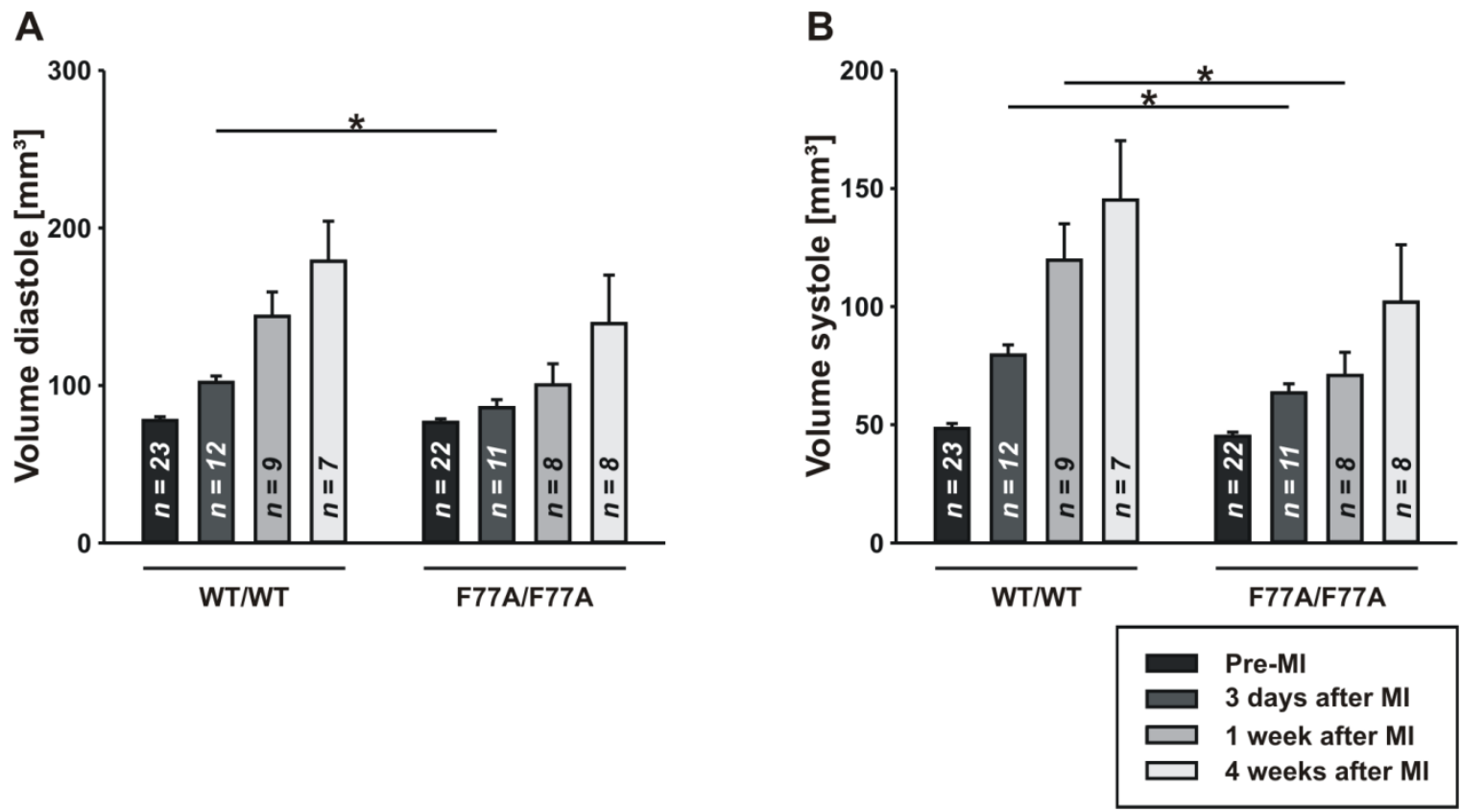

Figure 24: Increase in end-diastolic and end-systolic volume after myocardial infarction. The endsystolic volume $(A)$ and end-diastolic volume $(B)$ were calculated from the echocardiographic data obtained in B mode. For all mice analysed, pre-infarction data were acquired. Mice were analysed either during the acute phase on day 3 after infarction or kept for monitoring cardiac remodelling at 1 week and 4 weeks following myocardial infarction. Shown are only the data acquired from female mice. Numbers of mice included are indicated inside the columns. Asterisks indicate statistically significant differences. Error bars represent standard error of the mean.

Based on the anatomical measurements of left ventricular dimensions, end-diastolic and end-systolic volumes were computed (Figure 24). Following myocardial infarction, the left ventricle started to dilate massively, almost tripling its original volume by week 4 in the wildtype animals. In line with the above-mentioned data, enlargement of the left ventricle was reduced in the F77A knockin mice during the early phase on day 3 and week 1 and continued as a - however non-significant - trend up to week 4. Therefore, lack in IFN $\gamma$-responsiveness significantly ameliorated cardiac remodelling in females. 

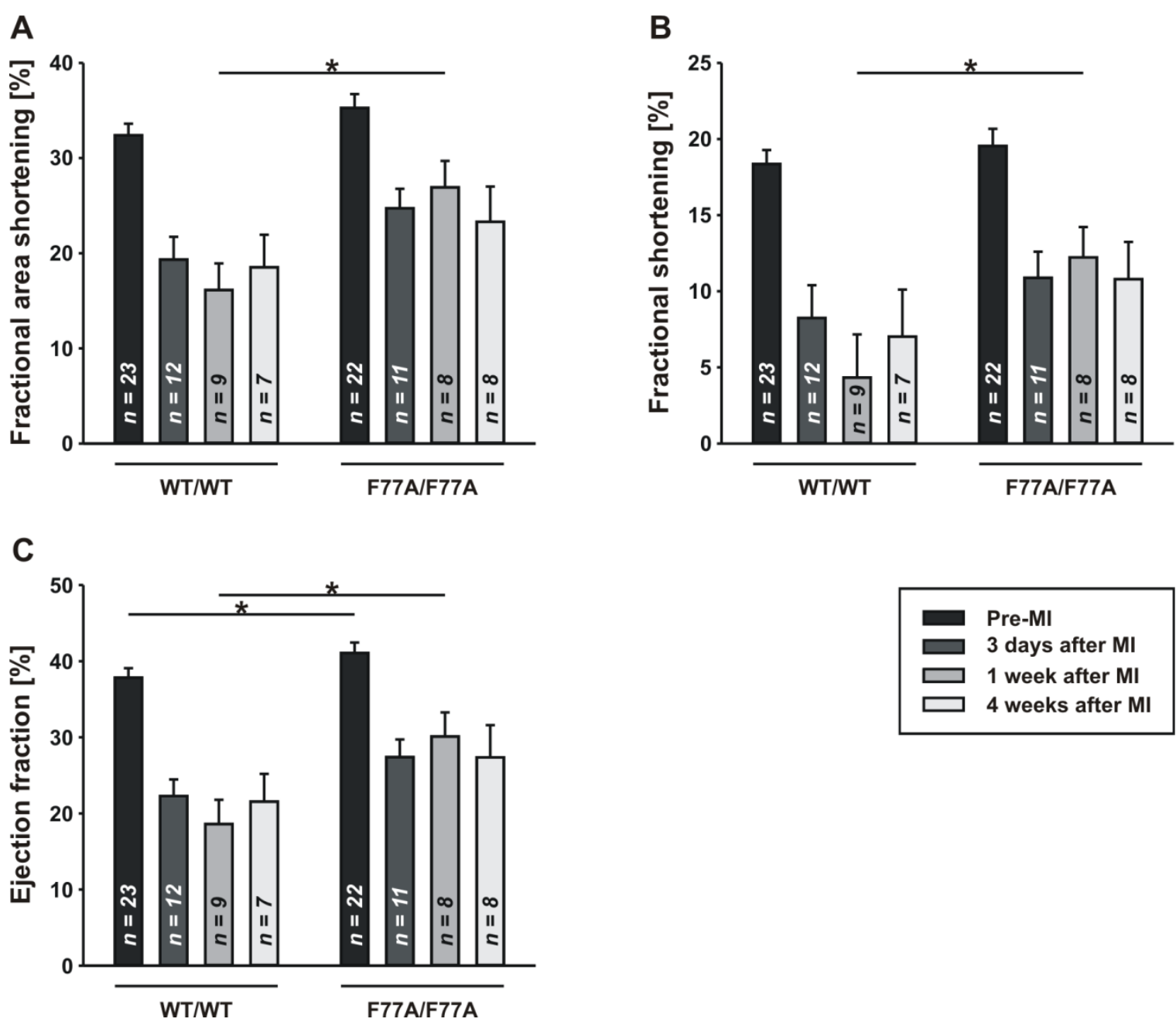

Figure 25: The reduced cardiac function after myocardial infarction is blunted in mice with impaired STAT1 tetramer stabilization. Fractional area shortening (A), fractional shortening $(B)$ and ejection fraction $(C)$ were computed from the echocardiographic data obtained in B mode. For all mice analysed, pre-infarction data were acquired. Mice were analysed either during the acute phase on day 3 after infarction or kept for monitoring cardiac remodelling on week 1 and week 4 after coronary ligation. Shown are only the data acquired from female mice. The numbers of mice included are indicated inside the columns. Asterisks indicate statistically significant differences. Error bars represent standard error of the mean.

In order to test the significance of these findings, fractional area shortening, fractional shortening and the ejection fraction were calculated (Figure 25). In response to the insult, cardiac function was markedly reduced as indicated by a pronounced and immediate drop in all three measurements. In STAT1-F77A female mice, cardiac function was ameliorated in comparison with the wildtype, 1 week after MI surgery. This trend was seen on a nonsignificant level on day 3 and week 4 after experimental infarction. Again, no significant differences were observed from the analysis of male mice. The lack of significant differences among the male cohorts might be attributed to the poor overall survival rates, as described above. Since only a few of the male mice survived, variance was high especially in the 
wildtype group, suggesting that animals with the highest impact of the infarction were not included in our analysis. Overall, the data revealed that the IFN $\gamma$-irresponsive STAT1-F77A mutant mouse line displayed ameliorated cardiac dilation and blunted remodelling resulting in better cardiac performance. This finding suggested that the absence of a functional IFN $\gamma /$ STAT1-dependent signal response was not only protective due to its direct nontranscriptional effect on apoptosis and autophagy (McCormick et al. 2012) but also due to other transcriptionally related mechanisms.

\subsubsection{STAT1 expression is reduced in hearts of STAT1-F77A knockin mice compared with the wildtype on day 3 following myocardial infarction}

Given the beneficial effects of the STAT1-F77A mutation in cardiac function during the acute phase of myocardial infarction, STAT1 regulation was then investigated at the molecular level. To this end, protein was extracted from the infarcted area of hearts that had been obtained and snap frozen 3 days after myocardial infarction. These lysates were compared with protein extracts isolated from unaffected areas of the basal parts of the ventricles and atria, as demonstrated by means of Western blotting. Figure 26A and 26B shows representative Western blots of protein samples from infarcted hearts of mice homo- and heterozygous for the STAT1-F77A mutation as compared with the wildtype. The phosphorylation levels of STAT1 and STAT3 as well as pan-STAT3 did not differ significantly between the three genotypes (Figure 26C, 26D and 26F). However, a significantly lower expression of STAT1 in the infarcted areas of the hearts of the STAT1F77A knockin mice was detected. This observation is in line with the overall reduced expression of STAT1 in the non-infarcted areas (Figure 26E). The expression of STAT3 is favoured in the myocardium of STAT1-F77A mice, as demonstrated by the increased ratio of total STAT3 to STAT1 which is not reflected on the phosphorylation level (Figure 26G and $26 \mathrm{H})$. 
A

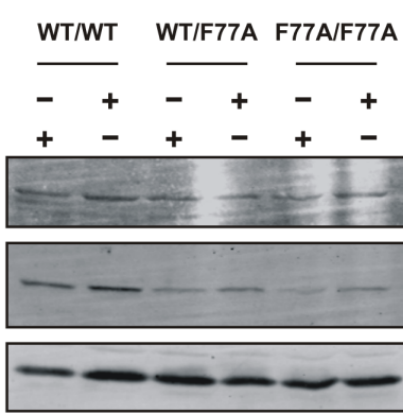

C
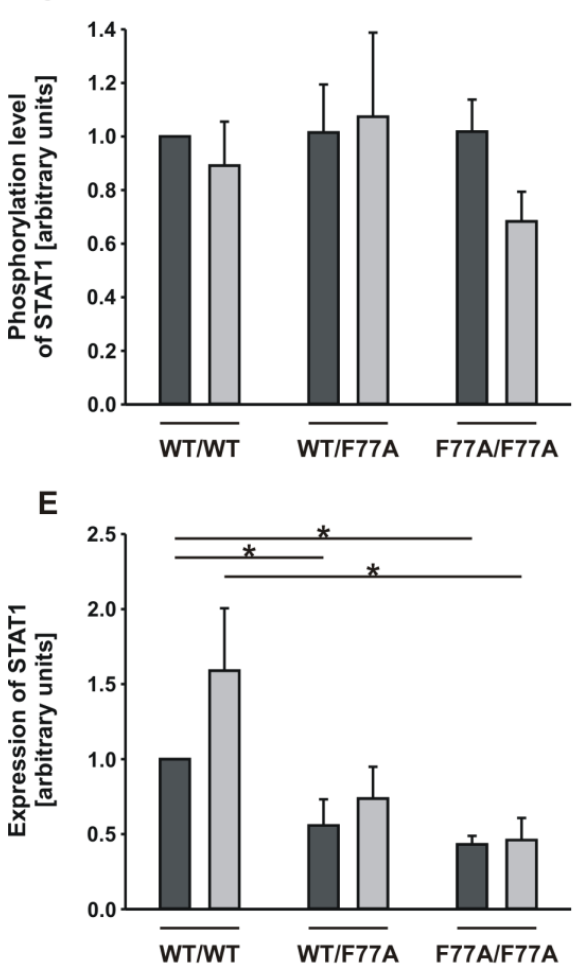

G

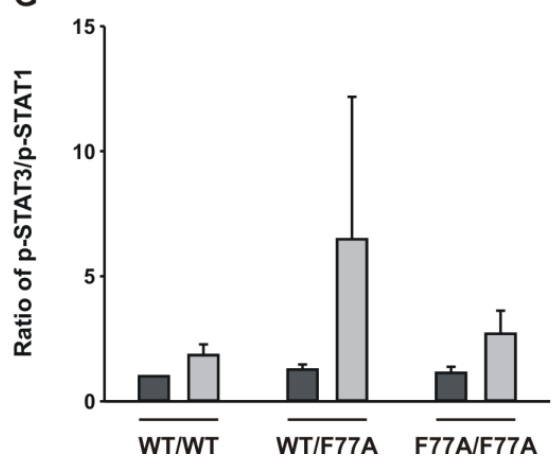

B

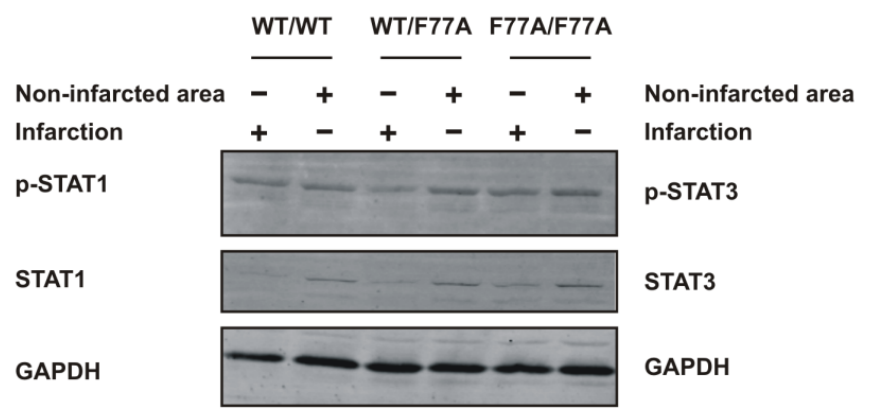

D
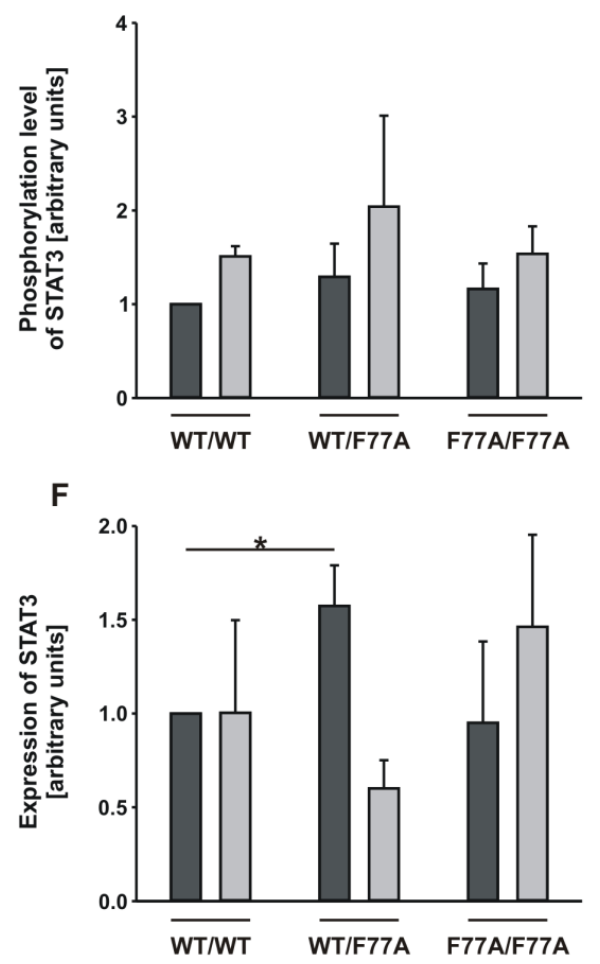

H

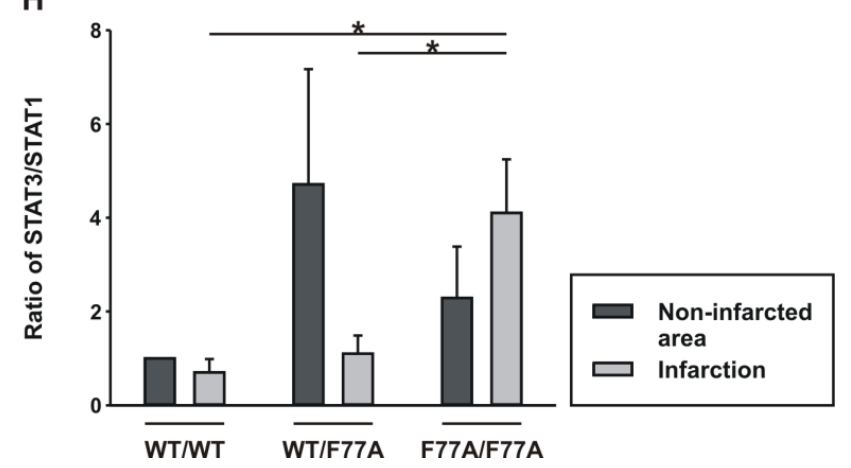

Figure 26: Expression and phosphorylation of STAT1 and STAT3 in heart tissue from STAT1F77A knockin mice on day 3 after myocardial infarction. (A) Representative Western blots of phosphorylated (p)-STAT1, STAT1 and GAPDH. (B) Representative western blots of p-STAT3, STAT3 and GAPDH. Densitometry analysis of phosphorylated STAT1 (C), phosphorylated STAT3 (D), and pan-STAT1 all normalized to GAPDH expression (E), as well as pan-STAT3 $(F)$, phospho-STAT3 to phospho-STAT1 $(G)$, and pan-STAT3 to pan-STAT1 (H). Data show means of four independent experiments. Only the $\alpha$-form of STAT1 and STAT3 were evaluated. Asterisks indicate statistically significant differences. Error bars represent standard error of the mean. 


\subsubsection{Immune histochemistry shows an accumulation of cells expressing tyrosine- phosphorylated STAT1 in the inflammatory infiltrate}

After assessment of the expression and activation of STAT1 and STAT3 using Western blotting, immunohistochemical staining was performed. Slides $(5 \mu \mathrm{m})$ of paraffin-embedded hearts obtained 3 days after myocardial infarction from wildtype C57BL/6N mice as well as mice heterozygous and homozygous for the STAT1-F77A allele were stained for tyrosinephosphorylated STAT1. Figure 27A shows representative images taken from hearts of myocardial infarction- and sham-operated animals from each genotype three days after surgery. Following sham operation, no phospho-STAT1-positive cells were observed in the cardiac muscle. However, following myocardial infarction, cells scattered throughout the infarcted area stained positively for tyrosine-phosphorylated STAT1. There was a tendency for phospho-STAT1-positive cells to be distributed at a low density over the infarcted area and to form clusters with a higher density distributed randomly within the infarcted area. Outside the inflammatory infiltrate, only few cells that were stained for phospho-STAT1, indicating that there is an association of STAT1 activation with the acutely infarcted area.

The accumulation of cells which stained positively for p-STAT1 was quantified using a score, considering two factors: their distribution over the ventricular wall and septum and, in addition, the density of phospho-STAT1-positive cells within this area. A score of "4" indicates the widest distribution and highest density, while " 0 " represents the absence of positive immunoreactivity. Figure $27 \mathrm{~B}$ and $28 \mathrm{C}$ show the results of these quantifications. Among the different genotypes, there was a marked difference in the distribution of the phospho-STAT1-positive cells, showing that, in the STAT1-F77A knockin mice, STAT1 phosphorylation was less restricted to confined areas. With respect to the density of p-STAT1-positive cells, there were no significant differences between the genotypes. This indicates that more cells staining positively for p-STAT1 were present in the infarcted area of cardiac sections obtained from STAT1-F77A knockin mice. These cells were distributed over a larger area of the myocardium but did not show stronger accumulation to confined regions when compared with the wildtype. 
A
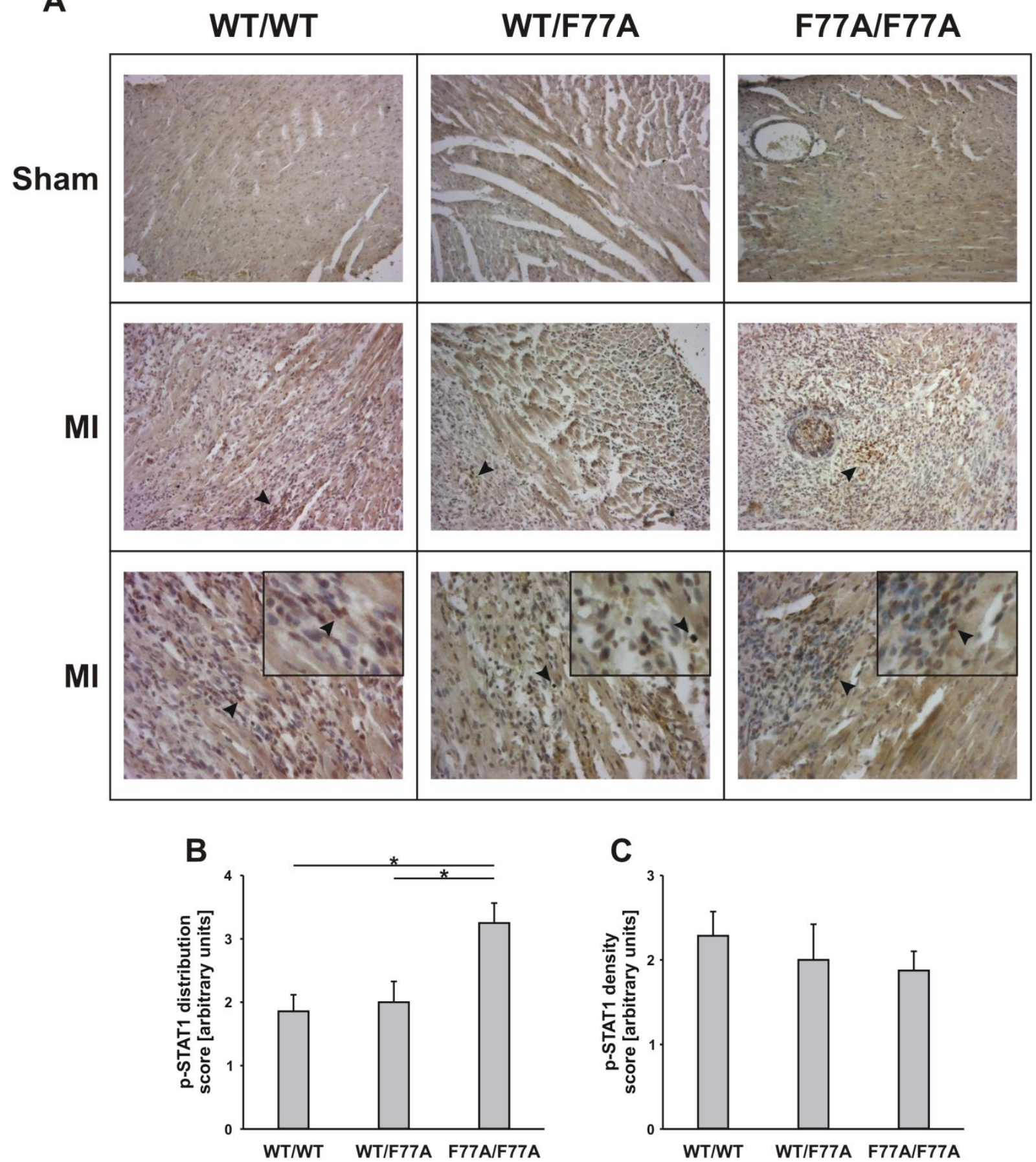

Figure 27: Staining of tyrosine-phosphorylated STAT1 in heart sections on day 3 after myocardial infarction or sham operation. (A) Representative microscopic fields of view of the left ventricular wall of sham-operated animals (top) or the infarcted area of MI-operated animals (middle and bottom) of all three genotypes are shown. In the infarcted areas, p-STAT1-positive cells are accumulated in clusters (arrowheads) with moderate accumulation outside these areas. The bottom row shows a higher magnification of p-STAT1 areas with staining of p-STAT1-expressing cells. $(B)$ Arbitrary quantification of the distribution of clusters of p-STAT1-positive cells over the ventricular wall and septum. (C) Arbitrary quantification of the density of p-STAT1-positive cells within the infarcted area. Asterisks indicate statistically significant differences. Error bars represent standard error of the mean. 


\subsubsection{No evidence of altered recruitment of neutrophils, monocytes or $\mathbf{T}$ cells to areas of myocardial infarction was observed in STAT1-F77A mutant mice}

A hallmark of ischemic injury of the cardiac muscle is an inflammatory reaction based on the recruitment of different immune cells. The main effectors in this context are neutrophil granulocytes, monocytes/macrophages, and T cells (Bonaventura et al. 2016). Whether the recruitment of one or all of these subsets was impaired in the absence of IFN $\gamma$ signaling was considered. FACS analysis of the lymphatic organs was performed to quantify baseline populations of inflammatory cell types in wildtype and STAT1-F77A knockin mice. For this purpose, the populations of $\mathrm{B}$ cells, $\mathrm{T}$ cells, neutrophils and monocytes were analysed in blood, spleen, lymph nodes and thymus in 9-week-old females. The analysis revealed a mild increase in regulatory $\mathrm{T}$ cells in spleen and lymph nodes (Figure 28). None of the other cell types showed significant differences with respect to their population size.
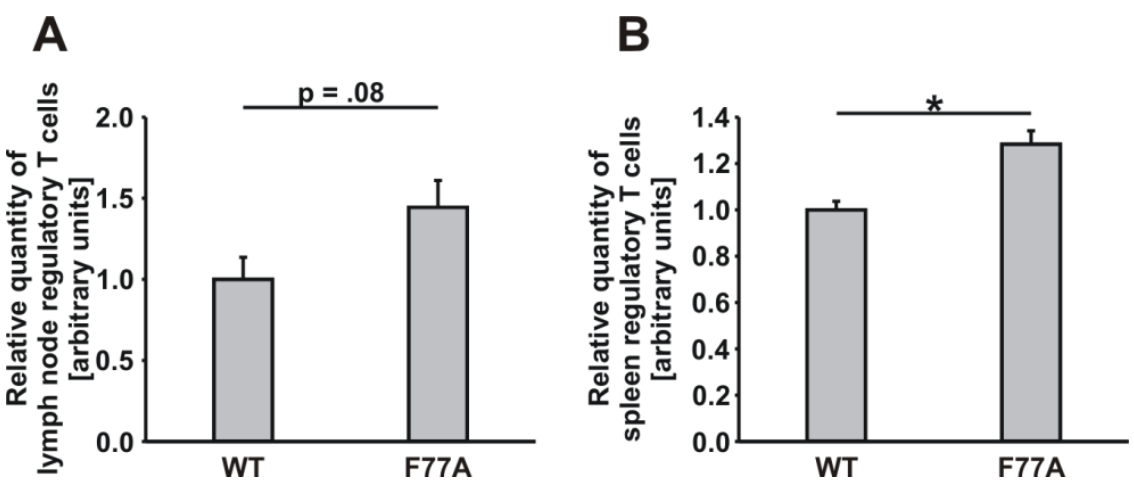

Figure 28: Analysis of the relative numbers of regulatory $T$ cells in lymph node and spleen of the STAT1-F77A mouse line. A: Analysis of regulatory $T$ cell populations in lymph nodes (A) and spleen (B) of STAT1-F77A knockin mice as compared to wildtype controls. Asterisks indicate statistically significant differences. Error bars represent standard error of the mean.

Figure 29 shows the typical expression patterns of two chemotactic cytokines in reconstituted U3A cells lacking endogenous STAT1 expression. The two STAT1-target genes CCL2 and CXCL1O display structurally different GAS elements in their promoter in which with the gene encoding CCL2 has a one-and-a-half GAS motif, while CXCL1O has one single GAS element. Both genes were induced less efficiently in the mutant despite almost normal expression levels at baseline. While CCL2 showed a reduced induction approximately by a factor of 10, CXCL10 induction had almost completely disappeared. 

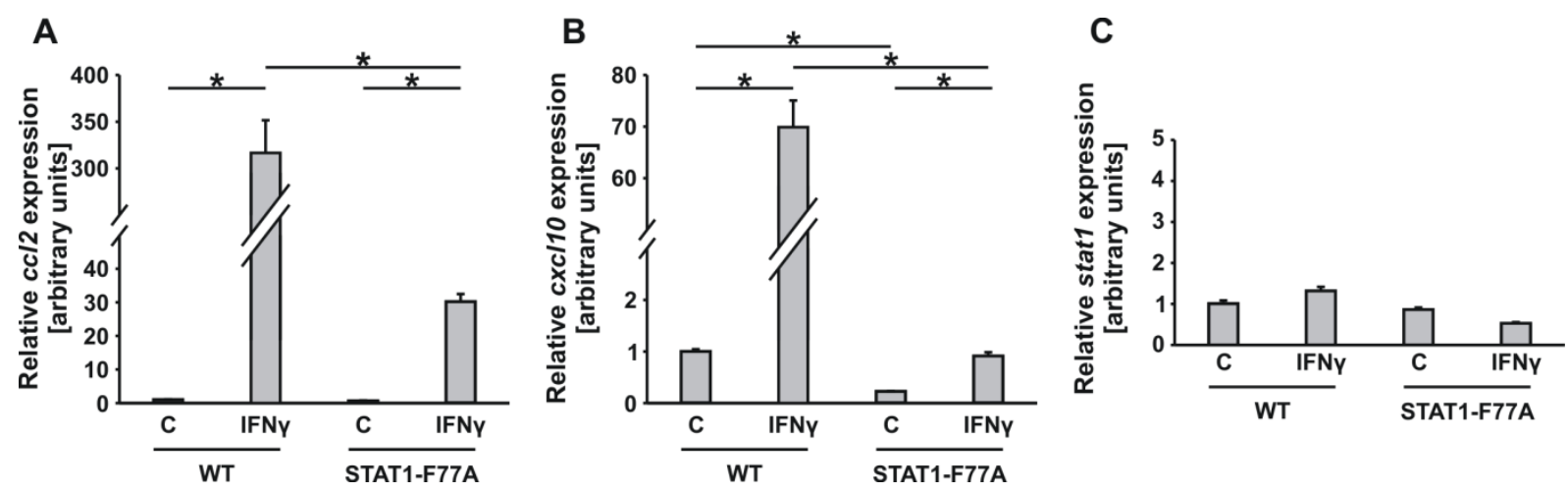

Figure 29: Reduced activation of two known IFNy-target genes by STAT1-F77A in reconstituted U3A cells. Shown are representative qPCRs of cDNA samples obtained from wildtype or STAT1-F77A expressing U3A cells. Untreated cells are compared with $6 \mathrm{~h}$ of IFN $\gamma$ treatment for the induction of the ccl2 (A), cxcl10 (B) and, as control statl (C) transcript. Asterisks indicate statistically significant differences. Error bars represent standard error of the mean.

Immunohistochemical staining using a horseradish-peroxidase-based method was utilised to identify different subsets of inflammatory cells during the acute phase of myocardial infarction. Staining for myeloperoxidase (MPO) was used as a marker for infiltrating neutrophils. The results identified neutrophils as the most prevalent cell type in the inflammatory infiltrates (Figure 30A). Quantification of the extent of myocardial infiltration by neutrophils revealed no significant differences in the overall area of infiltrates between the genotypes but a reduction in neutrophil density in animals carrying one but not two STAT1F77A alleles (Figure 30B and 30C).

In order to assess the presence of monocytes and macrophages, CD68 expression was detected (Figure 31). Compared with the population of neutrophils at the site of inflammation, CD68-positive cells made up a markedly smaller proportion of the inflammatory infiltrate (Figure 31A). However, when quantifying the distribution and density of monocytes and macrophages, no difference was detected between the genotypes (Figure 31B and 31C).

As the last subset, the infiltration of $\mathrm{T}$ cells was assessed through staining for CD3. Analysis revealed that this subset constitutes the smallest portion among the three subsets (Figure 32A). Again, quantification revealed that the cells were distributed equally between the genotypes (Figure 32B and 32C). In summary, immunohistochemical staining for the most prominent leukocyte subsets of the inflammatory infiltrate in the acute phase after myocardial infarction identified a small, yet non-significant reduction in the portion of neutrophils in mice homozygous for the STAT1-F77A mutation as compared to wildtype animals. This trend was support by a significantly lower density of neutrophils in the heterozygous mice compared with the wildtype. 

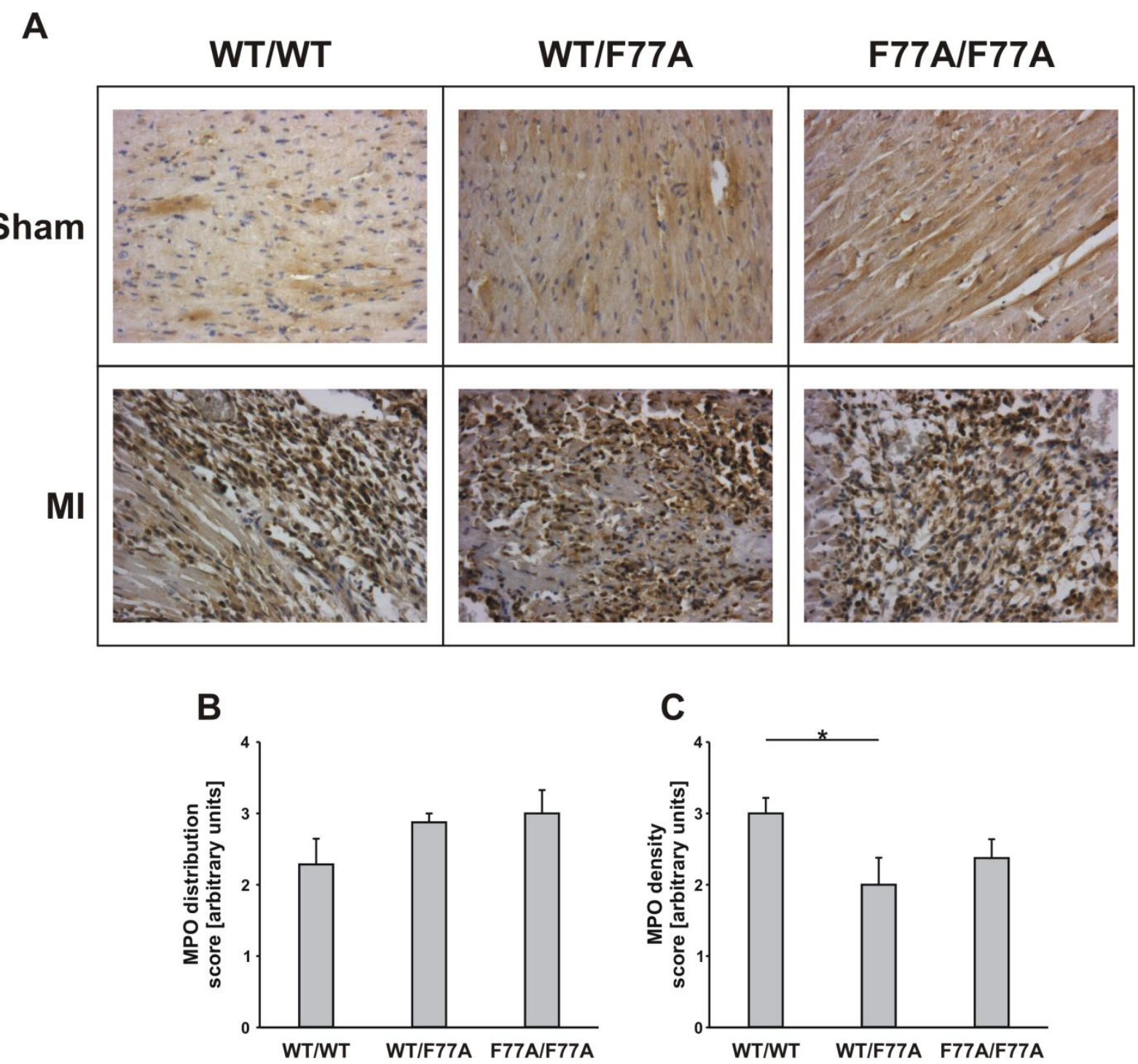

Figure 30: Staining of myeloperoxidase (MPO) on day 3 following myocardial infarction or sham operation. (A) Shown are representative fields of view of the left ventricular wall of sham-operated animals (top) and MI-operated animals (bottom) of all three genotypes investigated. Arbitrary quantification of MPO-positive cells with respect to their distribution over the ventricular wall and septum $(B)$ the density of MPO-positively stained cells $(C)$. Asterisks indicate statistically significant differences. Error bars represent standard error of the mean. 

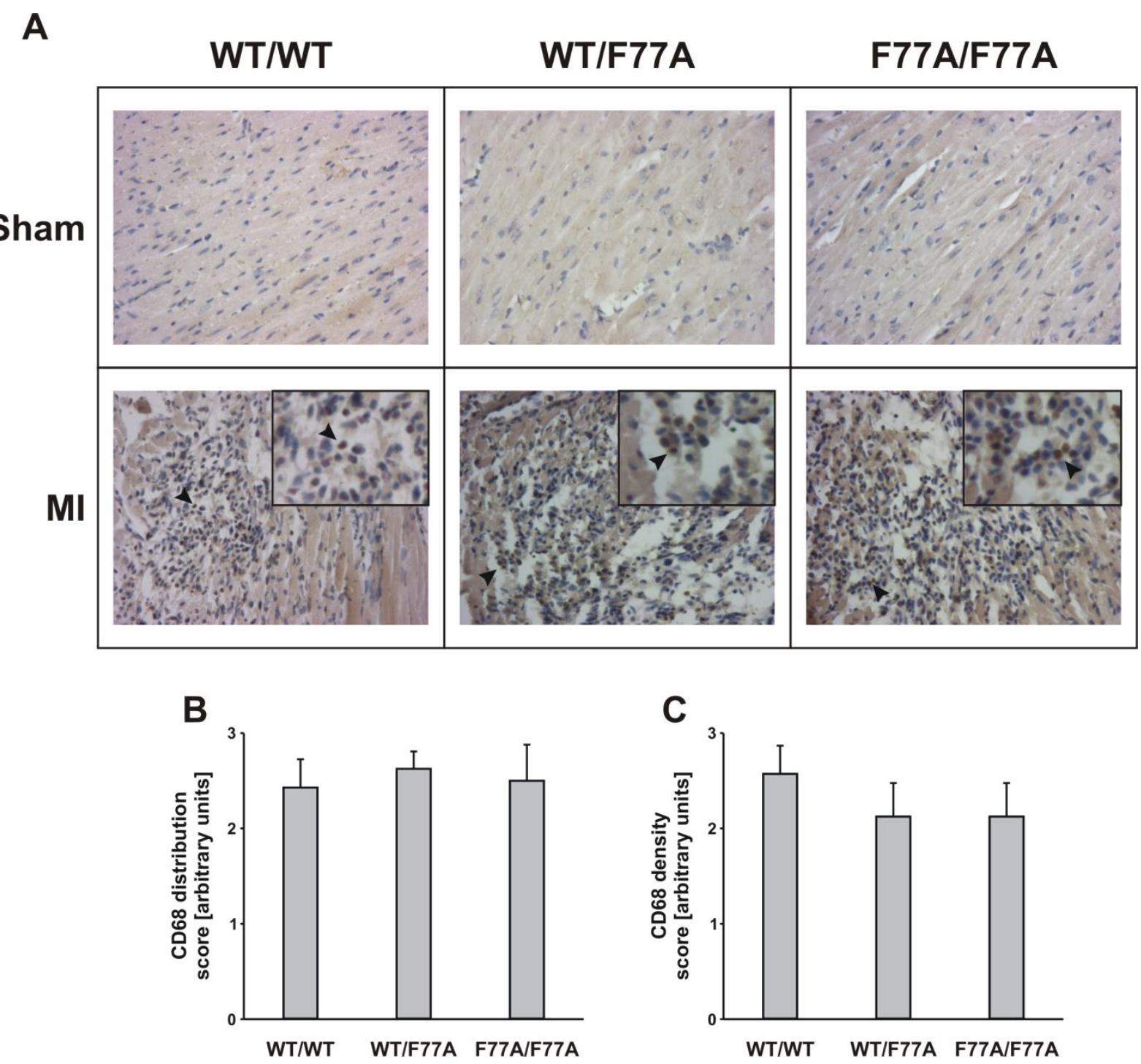

Figure 31: Staining of CD68 following ligation of the left ascending coronary artery or sham operation on day 3. (A) Shown are representative fields of view of the left ventricular wall of shamoperated (top) or MI-operated animals (bottom) of all three genotypes investigated. Single CD68positive cells were found in the inflammatory infiltrates (arrowheads) but not in the mock-operated ventricular wall. Arbitrary quantification of CD68-positive cells with respect to their distribution over the ventricular wall and septum $(B)$ and the density of CD68-positively stained cells $(C)$. Error bars represent standard error of the mean. 

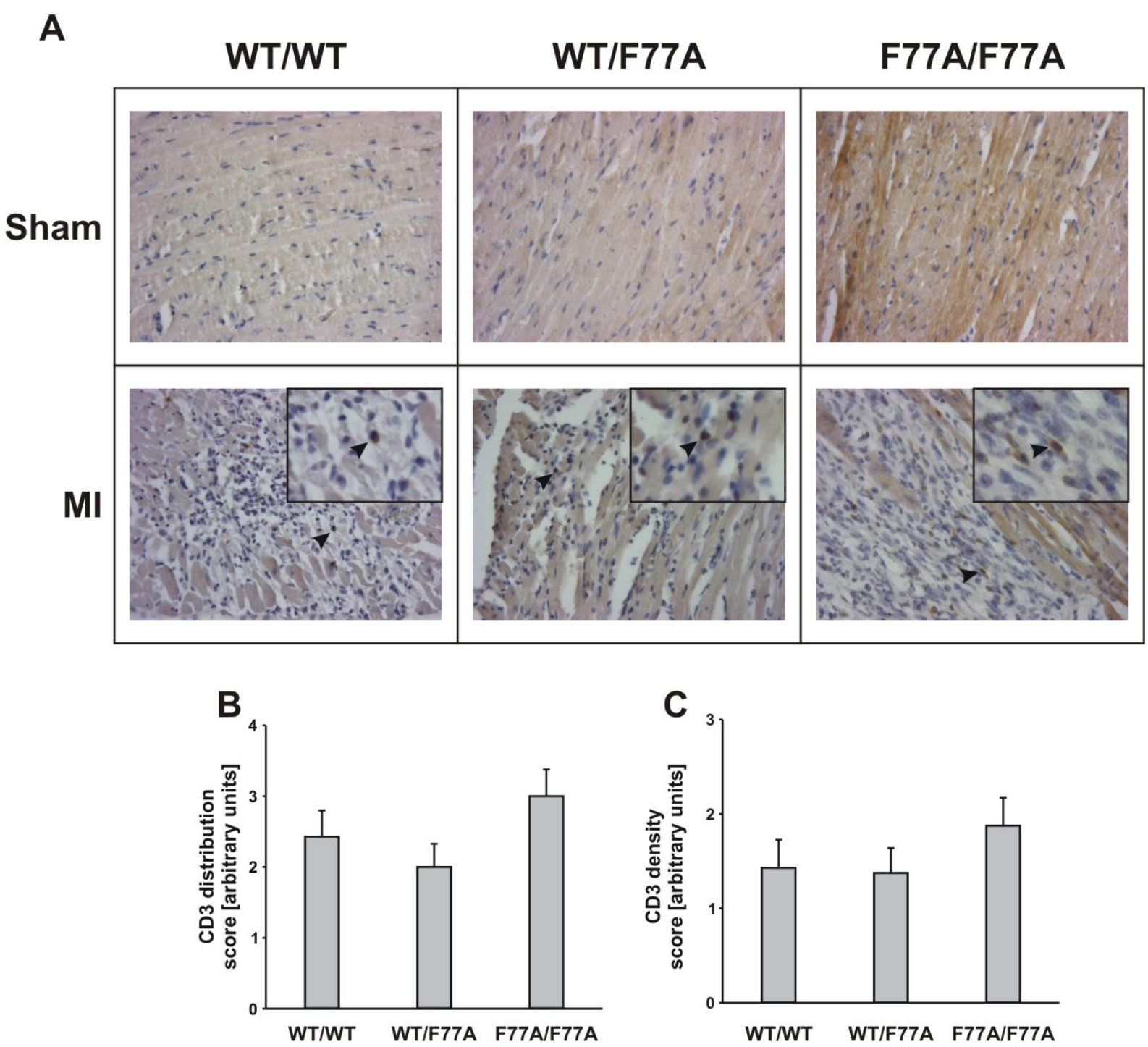

Figure 32: Expression of CD3 in infarcted heart tissue on day 3 follow-up. (A) Representative fields of view of the left ventricular wall of sham-operated animals (top) and mice subjected to myocardial infarction (bottom) of all three genotypes are shown. CD3-positive cells were found in the inflammatory infiltrate (arrowheads) but not in the ventricular wall of sham-operated animals. Arbitrary quantification of the distribution of CD3-positive cells over the ventricular wall and septum $(B)$ and the density of CD3-positive cells within the infarcted area $(C)$.

\subsection{STAT1 deficiency delays onset of EAE upon challenge with}

\section{lipopolysaccharide}

A plethora of studies has been published that show that STAT1 and STAT3 are main factors in the determination of $\mathrm{T}$ cell fate (Ma et al. 2016). Defects in these pathways have been associated with dysfunction of the immune system including immunodeficiency and autoimmunity. Within the context of experimental autoimmune encephalomyelitis (EAE), lack of STAT1 expression was shown to result in exacerbated disease progression and a 
higher rate of so-called atypical EAE (Bettelli et al. 2004; Peters et al. 2015). Studies on the impact of LPS on the onset and progression of EAE are rare and, depending on the exact time point of LPS application, a delay or exacerbation of the disease have been reported (Buenafe and Bourdette 2007; Park et al. 2015).

\subsubsection{STAT1 knockout mice are protected upon LPS challenge}

In this project, we aimed to discover the effect of a combination of $\mathrm{MOG}_{35-55}$ peptide-induced EAE and LPS-mediated activation of TLR4. Wildtype and STAT1-deficient mice both on a C57BL/6 genetic background were immunized following the standard protocol. Pertussis toxin was given at the time of immunization and $48 \mathrm{~h}$ later to increase the effectiveness of the challenge with the antigen. Half the mice cohort were given LPS i.p. ( $5 \mathrm{mg} / \mathrm{kg}$ body weight) $24 \mathrm{~h}$ after immunization. Following this protocol, the onset of EAE is expected between day 10 and day 12 after immunization.

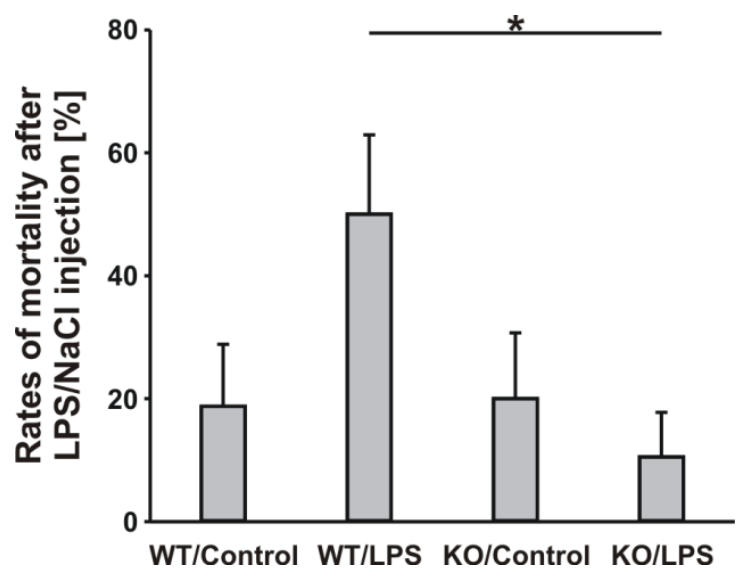

Figure 33: Survival before onset of EAE upon LPS challenge. Shown is the percentage of animals surviving the period between immunization with $M O G_{35-55}$ peptide and 10 days after immunization. Injection with LPS was performed i.p. on day 1 after immunization. Control animals were injected with an equal volume of $0.9 \% \mathrm{NaCl}$ solution. Asterisks indicate statistically significant differences. Error bars represent standard error of the mean.

The mean weight at the time of LPS challenge was $21.4 \mathrm{~g}$, and the mean age at $\mathrm{MOG}_{35-55}$ immunization was 11.3 weeks. Sixteen out of 66 animals, that had been immunized, died before the onset of EAE. Most of these animals belonged to the groups challenged with $5 \mathrm{mg} / \mathrm{kg}$ bodyweight LPS. This mortality was significantly higher in the wildtype than in the STAT1-deficient animals (Figure 33). The decreased sensitivity towards LPS challenge is in line with observations made for the STAT1-F77A mice in a previous study, also showing 
decreased mortality in the knockin model compared with their wildtype littermates (Begitt et al. 2014).

\subsubsection{Onset of clinical symptoms of EAE is delayed in STAT1 knockout mice injected with LPS}

The mean onset of EAE was 10 to 12 days after immunization with $\mathrm{MOG}_{35-55}$ peptide and progressed usually as an ascending flaccid paresis of tail and limbs. Disease severity was rated using a scale from $0-10$, where 0 corresponds to no symptoms, 7 to complete paresis of the hindlimbs, and 10 to death.

Figure 34 shows the mean onset and progression of EAE in STAT1-KO as compared to wildtype C57BL/6N mice that had been injected with LPS or with $0.9 \% \mathrm{NaCl}$ used as a control. In line with the literature, disease severity was mildly but not significantly increased in STAT1-deficient mice as compared to wildtype mice. Injection of LPS $24 \mathrm{~h}$ after immunization with $\mathrm{MOG}_{35-55}$ peptide resulted in a delay of disease onset by several days. This effect was even more pronounced in the group of STAT1-deficient mice injected with LPS (Figure 35). In terms of the disease severity, there were differences among the various treatment conditions: the NaCl-treated STAT1 knockout animals showed the most severe symptoms, while the LPS-treated wildtype animals presented with the mildest symptoms. The high variance is attributed to the high mortality rate upon LPS injection, which reduced the sample size of the cohort. In summary, the LPS-induced delay in the onset of EAE symptoms was equally pronounced in STAT1 knockout mice and wildtype animals. 


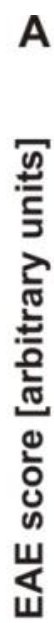

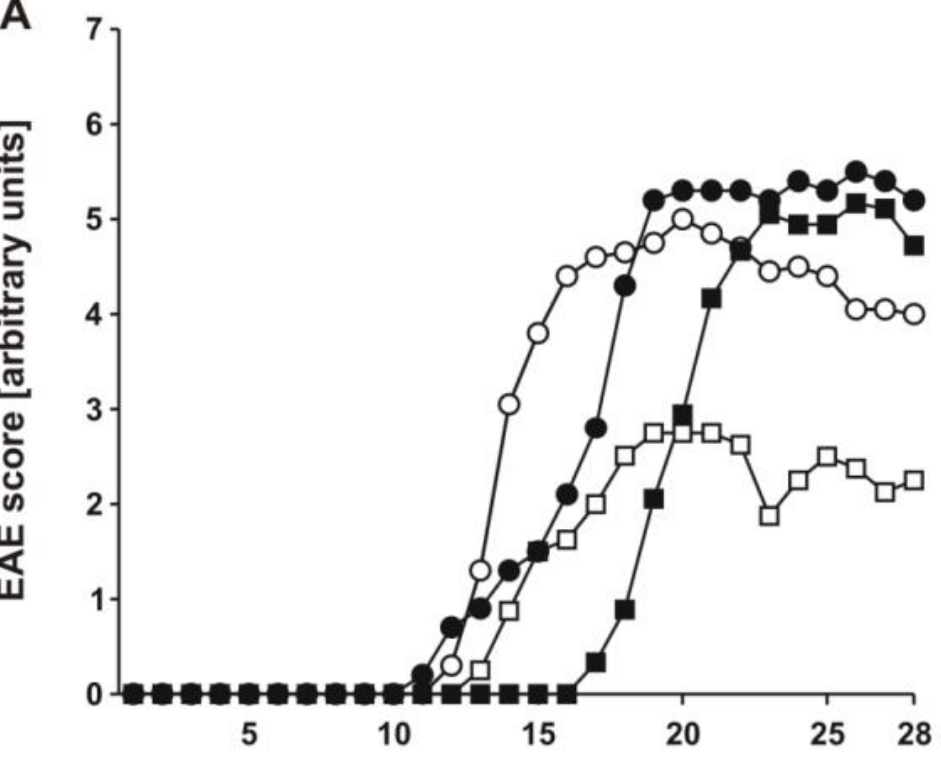

-O- WT control

$\rightarrow-\quad$ WT LPS

- KO contro

15

28

KO LPS

Time post immunization [days]
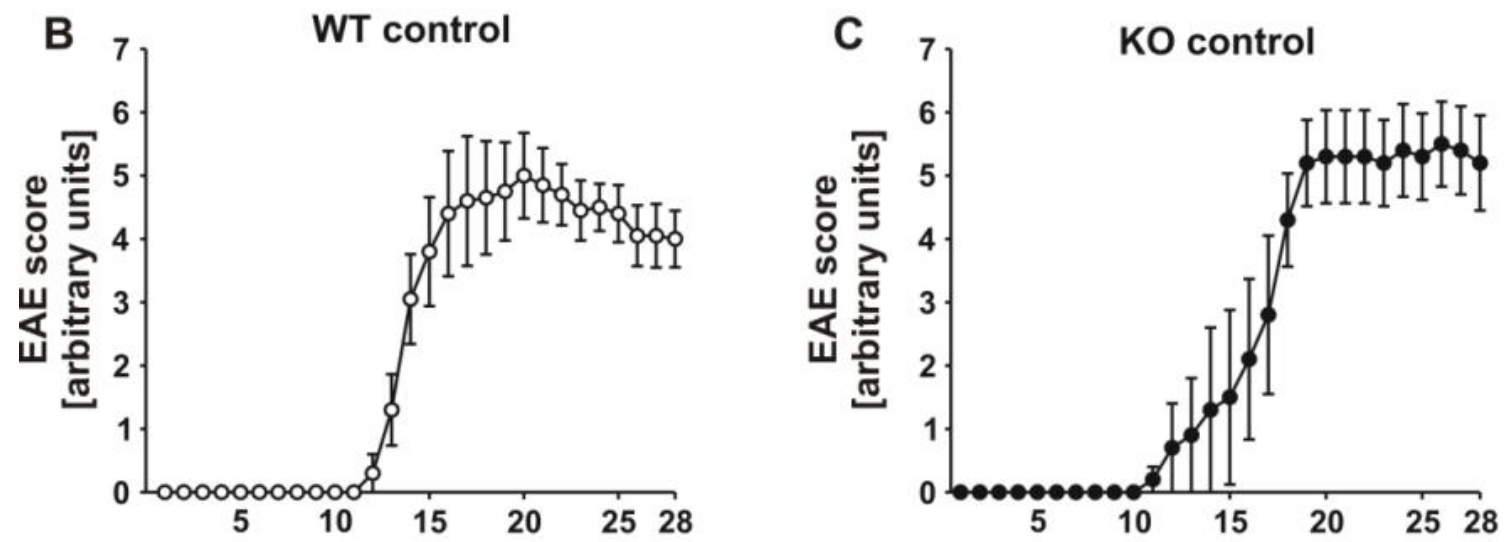

Time post immunization [days]

Time post immunization [days]
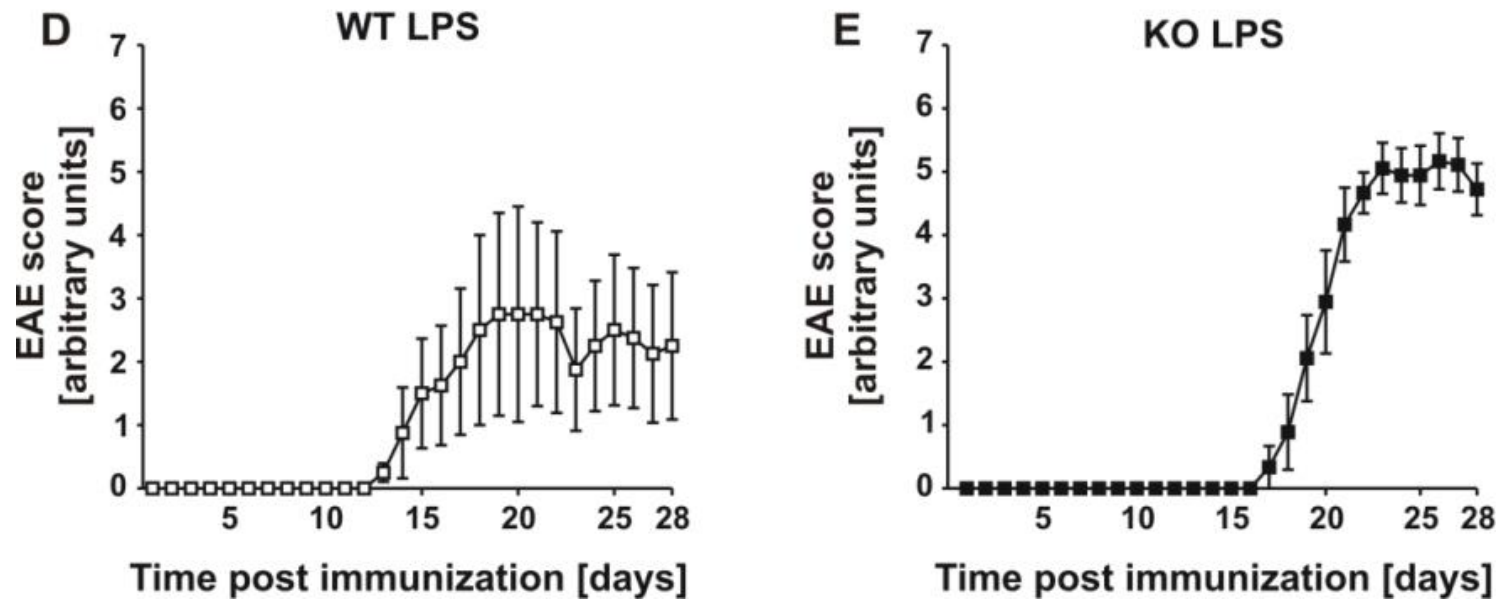

Time post immunization [days]

Figure 34: Clinical scores in STAT1-deficient and wildtype mice treated with and without LPS injection. (A) Combined scores of all four experimental conditions. Clinical scores of EAE progression rated on a 10-point scale in wildtype mice injected with $\mathrm{NaCl}$ solution (B), in STAT1 knockout mice injected with $\mathrm{NaCl}$ solution $(C)$, in wildtype mice injected with LPS (D), and in knockout mice injected with LPS (E). Error bars represent standard error of the mean. 


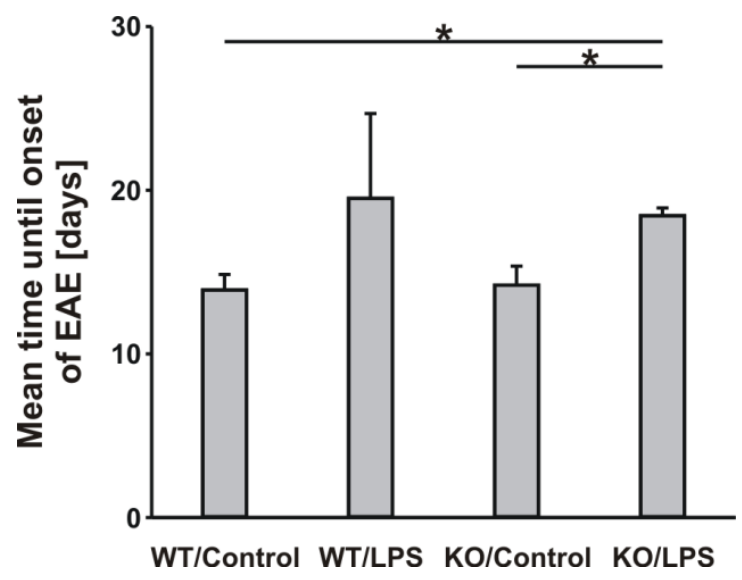

Figure 35: Mean date of EAE onset. Comparison between the four groups of wildtype (WT) and STAT1 knockout (KO) mice challenged with and without LPS after immunization for the induction of EAE. Asterisks indicate statistically significant differences. Error bars represent standard error of the mean.

\subsubsection{Cells from STAT1 knockout mice show increased proliferation upon $\mathrm{MOG}_{35-55}$ peptide stimulation}

EAE symptoms develop through the destruction of the myelin sheath of axons after invasion of the CNS by autoreactive immune cells. In order to provoke such a response, clonal expansion of antigen-specific $\mathrm{CD}^{+}{ }^{+} \mathrm{T}$ cells in the lymphatic organs is necessary (Rangachari and Kuchroo, 2013). To assess the proliferative capacity of immune cells derived from wildtype and STAT1-deficient mice, an ex vivo proliferation assay with $\mathrm{MOG}_{35-55}$ peptide was performed. Spleens and lymph nodes were extracted from mice of both genotypes which had been immunized with $\mathrm{MOG}_{35-55}$ peptide and then challenged with LPS or left untreated on day 10 and day 17 after immunization. Single cell suspensions produced from spleen and lymph nodes were incubated with either $\mathrm{MOG}_{35-55}$ peptide as a specific stimulus or concanavalin $\mathrm{A}$ as an unspecific stimulus for two days before addition of $\left[{ }^{3} \mathrm{H}\right]$-labelled thymidine for $16 \mathrm{~h}$ to evaluate proliferation through the readout of incorporated radioactivity.

Figure 36A shows the results of this proliferation assay for spleen-derived cells obtained 10 days after immunization. For the cell isolates from wildtype animals, there were only minor differences between LPS-challenged and unchallenged cells. When comparing cells from wildtype carriers with cells derived from STAT1 knockout animals and treated under the same conditions, a markedly higher proliferation rate in response to $\mathrm{MOG}_{35-55}$ peptide as well as concanavalin A was observed in the unchallenged cells. In contrast, cells obtained after LPS treatment displayed a markedly reduced response to $\mathrm{MOG}_{35-55}$ peptide, while the 
concanavalin A response remained strong. Similar results were obtained for cell isolates derived from the lymph nodes (Figure 36B), although these differences did not reach statistical significance.
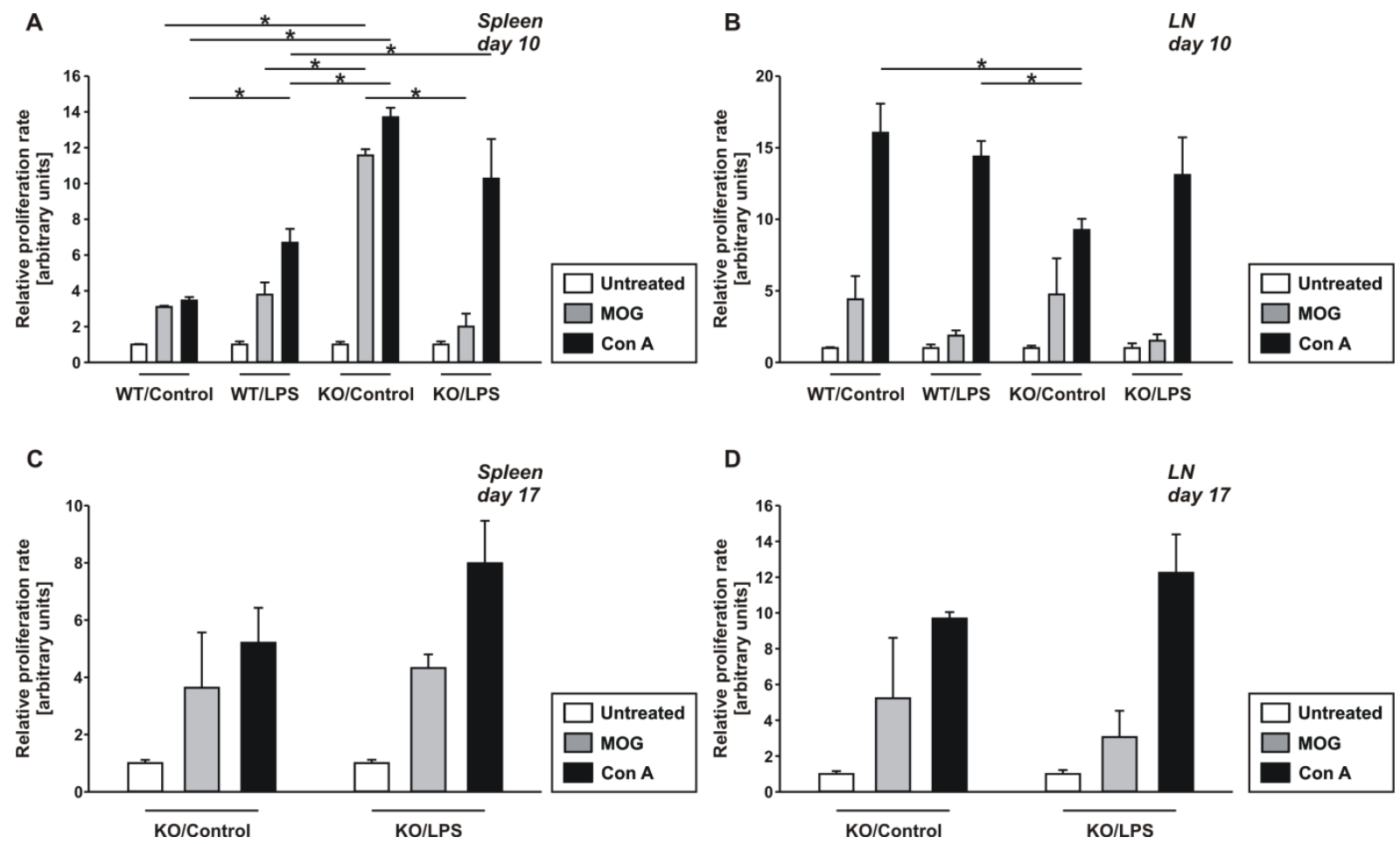

Figure 36: Relative proliferation rates of cells derived from lymphatic organs challenged with

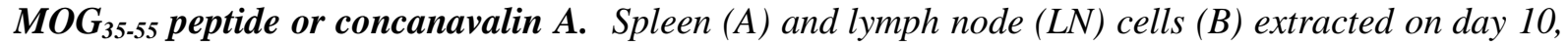
as well as spleen cells $(C)$ and lymph node $(D)$ cells extracted on day 17 were stimulated with $M_{35} 55$ peptide, concanavalin A (Con A) or left untreated. Proliferation was measured by incorporation of $\left[{ }^{3} \mathrm{H}\right]$-labelled thymidine for $16 \mathrm{~h}$. Asterisks indicate statistically significant differences. Error bars represent standard error of the mean.

Although LPS markedly delayed the onset of EAE in both wildtype and knockout mice, the reduced proliferation capacity in response to a specific antigen was more pronounced in the KO animals, while, in the wildtype animals, it remained at a similar level to that in the group of the untreated controls. The KO mice which had not been injected with LPS showed enhanced proliferation in response to the specific as well as the unspecific stimulus. Given the considerable differences between the cells derived from STAT1-deficient mice with and without LPS injection, a second proliferation assay was performed using cells obtained 17 days after $\mathrm{MOG}_{35-55}$ immunization. This time point was chosen since LPS delayed the onset of disease symptoms by about 1 week. Figure 36C shows the respective data of cells derived from the spleen. No significant differences were observed among the two conditions. These findings were supported by the lack of significant differences in the data obtained for the 
lymph node derived cells (Figure 36D). This indicates that the impact of LPS stimulation on lymphocyte proliferation was transient and in line with the in vivo observation that LPS injection delayed but did not reduce the severity in EAE in STAT1-deficient mice.

\subsubsection{STAT1 knockout cells produce increased amounts of IL-17A and IFNy}

A major mediator of the development of EAE is the population of $\mathrm{CD} 4^{+} \mathrm{T}$ cells which can develop towards different cell fates such as Th1, Th2, Th17 and regulatory T cells in response to signalling peptides (Bonelli et al., 2014). For the model of EAE in mice, Th1 cells and Th17 cells have been identified as the major actors in disease progression (Rangachari and Kuchroo, 2013). In order to identify whether the proliferative expansion of cells shown in Figure 36 was attributed to Th1 or Th17 cells, the secretion of marker cytokines of both cell lineages was quantified in the culture supernatant using ELISA technique. Figure 37 shows the results for the secretion of IL-17A, the marker cytokine of Th17 cells. Only low amounts of IL-17A were detected in the supernatants of cultured cells from spleen and lymph nodes of wildtype animals on day 10 (Figure 37A and 37B). The STAT1 knockout cells obtained from mice which had been immunized but not treated with LPS produced significantly higher amounts of IL-17A under $\mathrm{MOG}_{35-55}$ peptide or concanavalin A stimulation than cells from wildtype mice. This suggested stronger proliferation of Th17 cells and was in line with the more severe progression of EAE in this group. Cells derived from STAT1-deficient mice following injection with LPS secreted only low amounts of IL-17A in response to $\mathrm{MOG}_{35-55}$ peptide and concanavalin A.

ELISA analysis was repeated for cells derived from spleen and lymph nodes of STAT1$\mathrm{KO}$ animals sacrificed on day 17 after immunization (Figure 37C and 37D). In line with the observations made in the proliferation assays described above (Figure 36C and 36D), the differences between the LPS-treated and non-treated conditions disappeared at this later time point.

ELISA analysis was subsequently performed under similar experimental conditions to identify IFN $\gamma$ concentration in the ex vivo culture supernatant as a readout of Th1 proliferation (Figure 38). In the cells derived from untreated wildtype spleen, there was a marked secretion of IFN $\gamma$ in response to $\mathrm{MOG}_{35-55}$ peptide stimulation that exceeded the response to concanavalin A supporting the notion that Th1 cells are the main actors of EAE in this context (Figure 38A). In the cells derived from the LPS treated animals, the secretion of IFN $\gamma$ appeared to be lower although this effect was non-significant. Interestingly, there was a strong 
secretion of IFN $\gamma$ in the cells derived from STAT1 knockout animals treated with $\mathrm{MOG}_{35-55}$ peptide - the condition that showed the highest proliferation and also highest secretion of IL17A. Again, this effect was reversed in the cells derived from animals that had been injected with LPS. Data of the cells derived from lymph nodes confirmed these observations, although secreted levels of IFN $\gamma$ were much lower in the supernatants of these cultured cells and in some cases below the level of detection (Figure $38 \mathrm{~B}$ ).
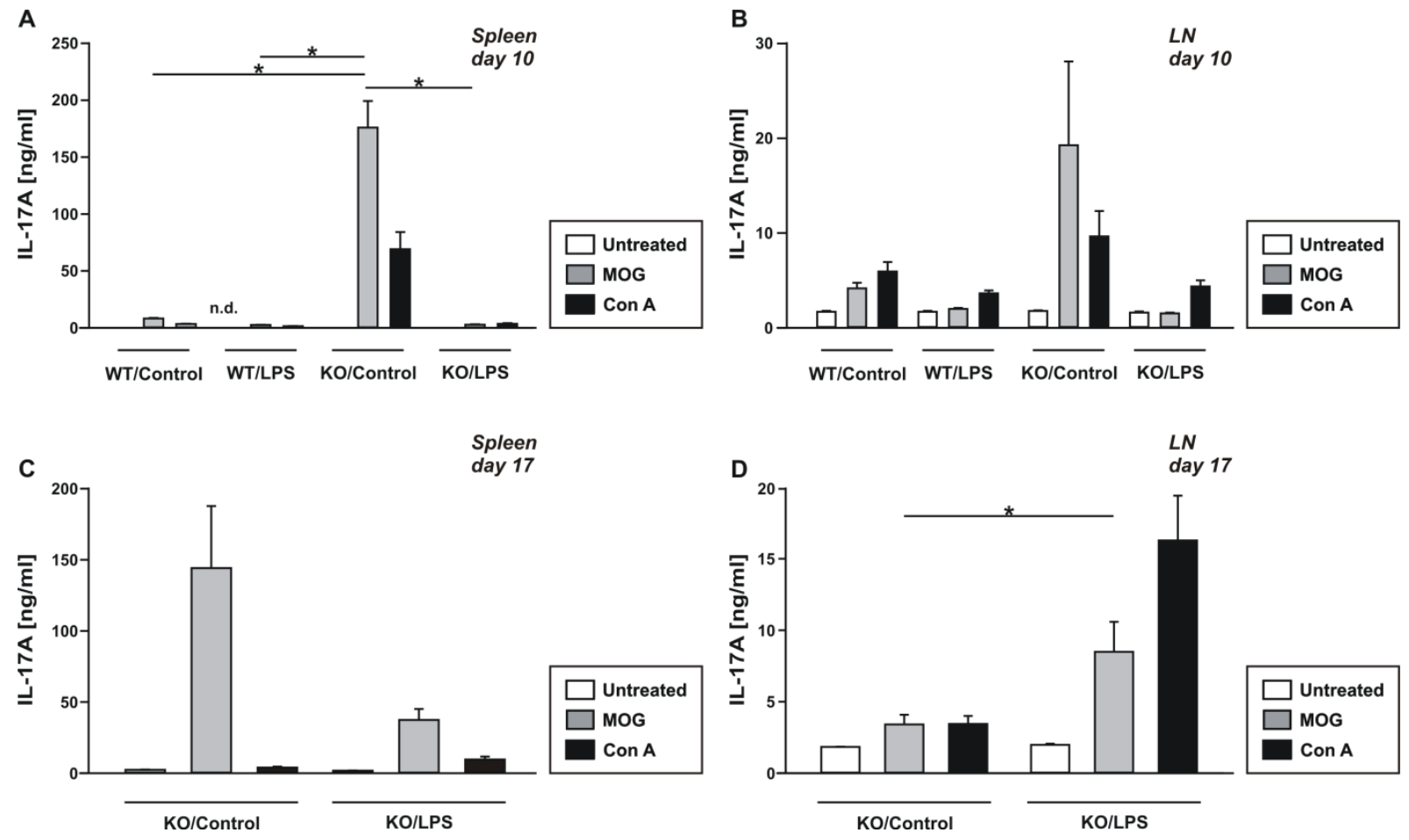

Figure 37: IL-17A concentrations in the supernatants of spleen and lymph node cells from $M_{O G}{ }_{35-55}$-immunised mice in the presence or absence of LPS challenge. Spleen cells $(A, C)$ and lymph node cells $(B, D)$ obtained on day $10(A, B)$ or day $17(C, D)$ after $M_{0 G_{35-55}}$ immunization of mice in the absence or presence of LPS injection were stimulated ex vivo with $M_{O G_{35-55}}$ peptide, concanavalin A (Con A) or left untreated. Production of IL-17A was measured using ELISA technique. n.d. marks measurements below the detection level. Asterisks indicate statistically significant differences. Error bars represent standard error of the mean.

The results of the later time point on day 17 post-immunization were in line with the previous observation that at this point, the differences between the STAT1-deficient animals injected with LPS or saline disappeared (Figure $38 \mathrm{C}$ and D). In summary, the secreted cytokine patterns supported the observations made from the proliferation assays and the clinical scoring for disease progression. 
A

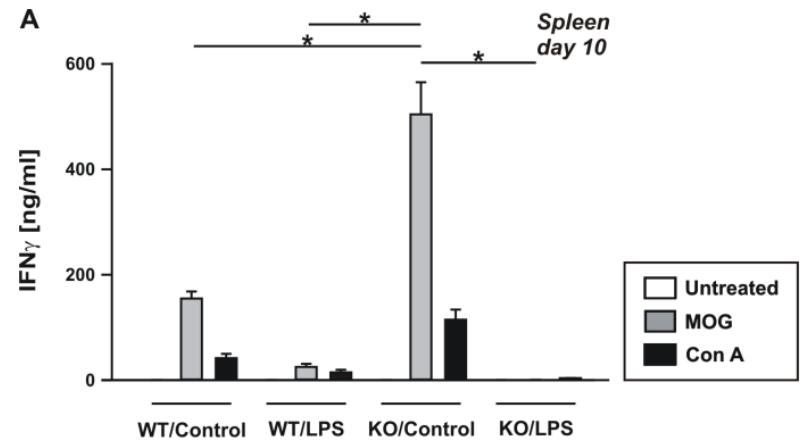

C

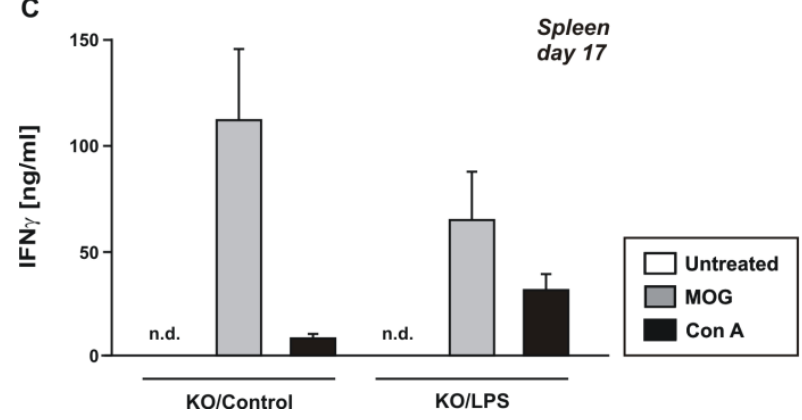

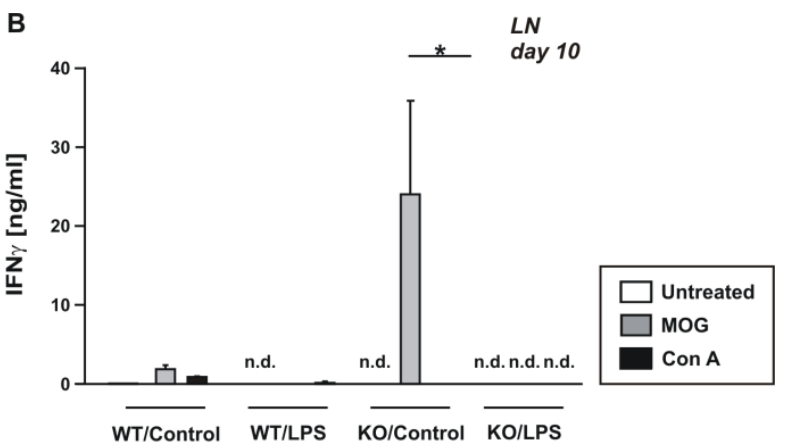

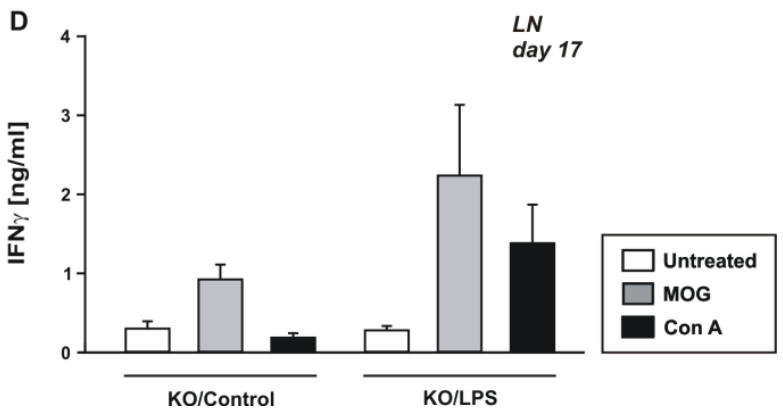

Figure 38: Dysregulated IFNy production in the supernatants of primary cells from lymphatic organs of STAT1 knockout animal cells immunized with $M O G_{35-55}$ peptide in the presence or absence of LPS co-stimulation. Spleen cells $(A, C)$ and lymph node cells $(B, D)$ obtained on day 10 $(A, B)$ or day $17(C, D)$ after $M O G_{35-55}$ immunization of mice in the absence (controls) or presence of LPS challenge. IFN concentrations in the supernatants of primary cells were detected using ELISA technique. n.d. marks measurements below the detection level. Asterisks indicate statistically significant differences. Error bars represent standard error of the mean. 


\section{Discussion}

Since the 1990s, STAT1 regulation has been under investigation as to its function in immunological processes (Shuai et al., 1992; Larner et al., 1993). Numerous studies have highlighted the essential role of the STAT1 transcription factor in defending viral, bacterial and parasite pathogens, and mutations in this protein have been shown to affect susceptibility to a catalogue of pathogens in mammals (Zhang et al., 2008). From crystallographic data, it is known that STAT1 can form a DNA-bound parallel dimer conformation as well as an antiparallel conformation not bound to DNA (Chen et al., 1998; Mao et al., 2005). Reciprocal interactions between $\mathrm{N}$-terminal domain dimers are required for stabilization of tetrameric complexes. To characterize the physiological consequences of impaired tetramer stability in an organismic context, a genetic knockin mouse line carrying the STAT1-F77A mutant allele has been described (Begitt et al., 2014). The phenotype of this mouse line shows decreased stabilization of tetramers, loss of cooperative DNA binding and an overall failure to induce IFN $\gamma$-regulated target genes. In this project, the impact of abolished STAT1 cooperative DNA binding was studied in a murine model of ischemic injury and the impact of a complete loss of STAT1-dependent signalling was analysed in the context of autoimmunity. Both pathological conditions are characterized by a pronounced inflammatory reaction which induces loss of functional tissue. The role of STAT1 in the regulation of rearrangements of the actin cytoskeleton in leukocytes through the ERM proteins will be discussed as a possible mechanism of leukocyte recruitment. The results underscore the fact that the STAT1-F77A mutant is a valuable tool for the understanding of STAT1 signal transduction and, in particular, for the study of the molecular mechanisms of the conformational transition from the parallel to the anti-parallel conformation.

The proposed mechanism of STAT1-dimer conformational shift, which is in line with data from the Vinkemeier laboratory (Wenta et al., 2008), relies on the constant dissociation and re-association of protomers as opposed to the hypothesis of $\mathrm{N}$-terminal interactions facilitating the conversion between the two conformational states of the STAT1 dimer (Mertens et al., 2006). An experimental set-up using EMSA was established to study the recombination between full-length untagged STAT1 and the GFP-tagged fusion protein thereof. The assay proved helpful in identifying inter-molecular interactions since it allowed for the identification of the two STAT1 variants based on an electrophoretic separation due to their different molecular weights (Figure 3). Both STAT1 variants demonstrated similar affinity and binding kinetics when interacting with consensus GAS elements, indicating that 
the carboxy-terminal fusion to GFP neither augments nor impairs the dissociation of tyrosinephosphorylated STAT1 dimers from their target sequence on DNA (Figure 4). Co-incubation experiments with untagged and GFP-tagged STAT1 showed that the two variants were able to form functional heterotetramers in the presence of specific GAS binding sites - a prerequisite to render them helpful in the study of the kinetics of the exchange of protomers (Figure 5 and Figure 7).

However, incubation of STAT1 dimers with DNA was shown to significantly slow down the kinetics of the protomer exchange which led to the notion that DNA binding protects from monomer exchange (Figure 9). Regarding the tetramer, the exchange of dimers was observed in the absence of DNA binding (Figure 7). It is worthy of note that reciprocal amino-terminal interactions facilitated by the critical phenylalanine residue in position 77 are dispensable for the dissociation into promoters, as shown by the fact that a STAT1 mutant carrying a mutation to alanine in this critical residue also showed dissociation (Figure 9 and Figure 10). Therefore, in contrast to its pivotal role in tetramer stabilization and cooperative DNA binding, no indication was found that the phenylalanine in position 77 was needed for the transition between the STAT1 conformational states or the exchange of protomers.

The data on the kinetics of the protomer exchange (Figure 9) when compared with the DNA binding kinetics (Figure 4) show that while the association and release from DNA occurs rather rapidly the in vitro half-life of phosphorylated STAT1 dimers at room temperature exceeds $10 \mathrm{~min}$. The structure of the phosphorylated STAT1 dimer was shown to be relatively stable even in the absence of DNA. High-affinity binding to its known target sequence further decelerates the exchange kinetics of STAT1 monomers. Furthermore, the results show that reciprocal interactions of the amino-termini of two adjacent monomers are not necessary for the formation of new dimer combinations.

As an alternative model for the reciprocal amino-terminal interactions facilitating the transition between the two conformational states of the STAT1 dimer (Mertens et al., 2006), a different mechanism had been proposed (Wenta et al., 2008). From the literature, it seems well established that the phosphorylation status of the critical tyrosine residue 701 is important for dimer orientation. While phosphorylated STAT1 preferentially acquires the parallel conformation, the absence of tyrosine phosphorylation stabilizes the more condensed anti-parallel conformation. Wenta and colleagues estimated a half-life of 20-40 min for each STAT1 dimer conformation which is of the same order of magnitude identified for the halflife of the protomer exchange kinetics measured in this project. This supports the hypothesis 
that conformational change might be achieved by dissociation and re-association in different conformational states.

The acquired data cannot exclude the possibility that the phosphorylated STAT1 dimer is constantly oscillating between the two different conformational states at a rate much faster than the kinetics identified for the exchange of protomers and that this oscillation might be supported by amino-terminal interactions. However, this putative oscillation cannot be the rate limiting step in the dimer dissociation kinetics nor the main determinant of dimer stability.

The physiological significance of these observations remains to be evaluated. It is assumed that the anti-parallel STAT1 dimer is the substrate for the inactivating STAT1-specific phosphatase, since binding of STAT1 to DNA hinders dephosphorylation (Meyer et al., 2003; Mertens et al., 2006). The conformational change from the parallel to the anti-parallel state is a prerequisite for STAT1 inactivation. Mutant isoforms of STAT1 such as F77A which are discussed to specifically hinder the alignment in the anti-parallel orientation have been found to be associated with prolonged tyrosine phosphorylation (Zhong et al., 2005; Mertens et al., 2006).

A study using single-molecule microscopy has shown that phosphorylated STAT1 associates to the DNA in a way that alternates short binding events of up to $5 \mathrm{sec}$ with distinct saltatory movements (Speil et al., 2011). This process was interpreted as a series of intermitting events of retardation and immobilization by putative STAT1-binding sites. These data support the observation from this study that DNA-dimer interactions of STAT1 are less stable and characterised by a shorter half-life than intradimer interactions. Therefore, the dimer dissociation kinetics could be the rate limiting step in the STAT1 activation cycle.

The findings support the hypothesis that constant dissociation and re-association of phospho-STAT1 dimers occurs. However, the kinetics of the protomer exchange appears to be relatively slow in comparison with the fast release of dimers from DNA suggesting that the dissociation kinetics is the rate limiting step in the STAT1-mediated transcriptional response within the nucleus. The results from this thesis clearly demonstrate that high-affinity binding to DNA hinders monomer exchange and that intradimeric interactions between the N-termini are dispensable for the protomer exchange.

The focus of this project was then directed towards the regulation of the expression of the EZR gene and whether STAT1 is playing a role in this context. Several reports have identified 
components of the JAK/STAT pathway as mediators in actin cytoskeleton rearrangements during inflammatory processes and one way to induce stimulus-dependent cytoskeletal alterations is by ezrin signalling. Up to now, the focus of most of these studies had been on immediate changes, mostly via Rho kinase signalling. In macrophages, IFN $\gamma$ was shown to increase the fraction of filamentous actin and Rac1-GTP which enhances the phagocytosis of bacteria (Frausto-Del-Río et al., 2012). Another study demonstrated that acquisition of an M1 or M2 type by macrophages is linked to the reorganization of the cytoskeleton. In vitro differentiation experiments have revealed that M1 macrophages, as induced by IFN $\gamma$ and LPS, are round and flat in shape, while M2 macrophages, as induced by IL-4 and IL-13, are elongated and show a more polarized phenotype. When being grown on a surface that strongly favours an elongated cell shape, the M2 lineage was favoured even in the presence of LPS and IFN $\gamma$ stimuli showing that cell shape and cytoskeletal structure help determine macrophage cell fate (McWhorter et al., 2013). In T cells, signalling induced by CXCL12 and CCL21 triggers dephosphorylation of ERM proteins and increases motility of the cells. Surprisingly, knockdown of JAK1 and JAK2 inhibited ERM phosphorylation and impaired chemokine-induced migration suggesting that JAK/STAT signalling can influence ERM metabolism (Pérez-Rivero et al., 2013).

Unlike the moesin and radixin gene, the gene encoding ezrin carries a GAS motif near the transcription start site (Table 23 and Table 24). In humans, this GAS motif possesses a unique structure since two optimal STAT1-binding sites are located approximately $500 \mathrm{bp}$ upstream of the transcription start site in tandem orientation, separated by only $1 \mathrm{bp}$. With these characteristics, the GAS motif of the EZR gene markedly differs from the one-and-a-half GAS site of the CCL2 gene which has been suspected of being more sensitive to changes in STAT1 phosphorylation levels and could therefore be affected to a higher degree than most STAT1target genes by just minor increases in STAT1 phosphorylation (Hüntelmann et al., 2014).

The EMSA experiments performed in this project using the motif from the human EZR gene support the conclusion that this sequence can only be occupied by a single STAT1 homodimer (Figure 11). No preference for either of the two GAS elements aligned in tandem orientation was observed. This finding suggests that the putative GAS elements in the promoter of the human EZR gene are a target sequence for STAT1 homodimers which can only bind one dimer specifically most probably for steric reasons. Since most STAT1-target genes contain only one GAS motif in the vicinity of the TSS (Decker et al., 1997), the role of the putative STAT1 target sequence of the EZR gene was studied in the context of living cells. 
ChIP experiments in Jurkat cells revealed that STAT1 binds to the EZR promoter in these cells (Figure 12). Since this human cell line was derived from a T cell leukaemia (Gillis and Watson, 1980), the binding of STAT1 to the EZR promoter not only suggests a role in gene regulation but might also indicate that this is of relevance in $\mathrm{T}$ cell function. However, since ezrin expression and activation have been found in connection with carcinogenesis (Leiphrakpam et al., 2014), induction of the EZR promoter might also be due to the transformed nature of this cell line.

Real-time PCR of reversely transcribed RNA extracted from Jurkat as well as HL-60 cells consistently revealed a significant increase in ezr mRNA expression by the factor 2.5 in response to IFN $\gamma$ treatment (Figure 13 and Figure 14). This effect was less pronounced in response to stimulation with IFN $\alpha$. Both interferons induce the phosphorylation of the STAT1 homodimer but in response to IFNa, a fraction of the STAT1 monomers becomes also sequestered into ISGF-3 (Horvath, 2000). Since no ISRE-binding site has been identified for the EZR promoter, the reduced induction below the level of significance in response to IFN $\alpha$ is in line with the hypothesis that EZR is an exclusive target gene of IFN $\gamma$-mediated STAT1 signalling. The observation that the induction of ezrin transcription is relatively mild might be attributed to the fact that ezrin is a cytoskeletal protein which only needs induction by a low magnitude and is moderately expressed at baseline. In contrast, most other IFN $\gamma$-regulated genes are cytokines, transcription factors and effectors of immune response which are close to silent at baseline and induced at high magnitudes to protect the tissue from infections by pathogenic agents.

Surprisingly, the induction of ezrin expression was not observed on the protein level, neither in leukaemia-derived tumour cell lines nor in bone marrow lysates obtained from wildtype mice, STAT1-deficient mice and mice which expressed the STAT1-F77A mutant (Figure 15, Figure 16, Figure 17, and Figure 18). This suggests that STAT1-induced changes in the protein expression of ezrin are below the detection level or that a different mechanism counteracts the weak induction of ezr mRNA production. A candidate for this effect would be the protease calpain. In response to extracellular stimuli, calpain actively cleaves ezrin in a $\mathrm{Ca}^{2+}$-dependent manner (Yao et al., 1993). Calpain inhibition resulted in massive overexpression of ezrin and mild overexpression of radixin and moesin in NIH-3T3 cells. By overexpressing these ERM proteins, calpain inhibition led to an impairment in the cytoskeletal rearrangements necessary in cell spreading (Potter et al., 1998). Studies in the myeloid leukaemia-derived cell lines THP-1 and U937 have shown that IFN $\gamma$ upregulates 
mRNA and intracellular protein levels of calpain expression (Deshpande et al., 1995). Therefore, increase in calpain expression may be a possible mechanism to counterbalance STAT1-induced ezrin transcription.

Besides the regulation of ezrin on the transcriptional level, whether IFN $\alpha$ - or IFN $\gamma$-induced signalling could evoke phosphorylation of ERM proteins was evaluated. Threonine phosphorylation of the ERM proteins has been shown to regulate their activation status. The Rho kinases, a family of small GTPases, were described to phosphorylate ezrin at Thr567, radixin at Thr564, and moesin at Thr558. Phosphorylation of this conserved residue reduces head-to-tail associations of the amino-terminal and the carboxy-terminal domains (Matsui et al., 1998). Interactions of ERM proteins and small GTPases have been implicated in a variety of ERM-dependent mechanisms including polarization, adhesion, migration and $\mathrm{T}$ cell homing (Okamoto et al., 1999; Menager et al., 1999; Denker and Barber, 2002; CernudaMorollón et al., 2010; Ishihara et al. 2015). IFN $\gamma$-activated signalling mediates internalization of tight-junction proteins in a RhoA/ROCK-dependent manner, thereby impairing epithelial barrier integrity (Youakim and Ahdieh, 1999; Utech et al., 2005). Therefore, phosphorylation of ERM protein in response to interferon treatment was monitored.

In HL-60 cells, ERM phosphorylation levels were comparatively low and showed no induction by interferon treatment over different durations of time ranging from $10 \mathrm{~min}$ to $24 \mathrm{~h}$ (Figure 16). In protein lysates obtained from murine bone marrow, phosphorylation of ezrin and moesin was readily detectable. Treatment of single cell suspensions derived from murine bone marrow with IFN $\alpha$ or IFN $\gamma$ for $45 \mathrm{~min}$, produced no significant changes in phosphorylation levels of ERM proteins (Figure 18). Therefore, the observations do not support the hypothesis that interferon-induced signalling directly affects ERM phosphorylation.

The STAT1-F77A mutant included in these experiments was linked to poor induction of IFN $\gamma$-mediated gene expression due to a lack in tetramer stabilization which is pivotal for cooperative DNA binding of STAT1 (Meyer et al., 2004; Begitt et al., 2014). This mutation has impact on the susceptibility towards infectious disease, as the STAT1-F77A mouse is protected from vesicular stomatitis virus but showed higher mortality upon infection with Listeria monocytogenes. These data are in line with the notion that most genes which are upregulated as a response to viral infection are mediated by type I interferons via the ISGF-3 and that lack in binding cooperativity has little impact on ISGF-3 association to ISRE-binding sites (Meyer et al., 2004; Begitt et al., 2014). The phenotype of the STAT1-F77A mouse has 
so far only been studied in infectious diseases. Here, for this project, its phenotype in the context of myocardial infarction was evaluated.

Over the last two decades, STAT proteins have been attributed a role in myocardial infarction and ischemia/reperfusion injury (Fuchs et al., 2003; Hilfiker-Kleiner et al., 2004; Boengler et al., 2008; Fischer and Hilfiker-Kleiner, 2007; Fischer and Hilfiker-Kleiner, 2008; Hilfiker-Kleiner et al., 2010; McCormick et al., 2012; Knight et al., 2012). Ischemia has been shown to promote apoptosis in an IFN $\gamma / \mathrm{STAT} 1$-dependent manner by inducing the expression of caspase 1 in cultured neonatal cardiomyocytes as well as in the murine heart using the Langendorff perfusion method (Stephanou et al., 2000). For the promotion of apoptosis in cardiomyocytes, the most important role was attributed to the STAT1 C-terminal domain since expression of a truncated mutant lacking the last 60 amino acids resulted in decreased rates of apoptosis. This effect was not found to be directly regulated via STAT1 transcriptional activity, because lack of the DNA-binding domain did not impair the proapoptotic actions of a STAT1 mutant (Stephanou et al., 2002). STAT1 and STAT3 have both been shown to become phosphorylated in response to ischemic injury to the myocardium. Different antioxidant agents which are known to be cardioprotective were shown to inhibit STAT1 activation (McCormick et al., 2006; Scarabelli et al., 2009). Given the function of STAT3 in mitochondrial respiration (Wegrzyn et al., 2009; Carbognin et al., 2016) STAT3 is known to have cardioprotective effects under pressure overload as well as during ischemia/reperfusion injury (Fuchs et al., 2003; Hilfiker-Kleiner et al., 2004; Boengler et al., 2008; Fischer and Hilfiker-Kleiner, 2007; Fischer and Hilfiker-Kleiner, 2008; Heusch et al., 2011). A lack of STAT3 expression in cardiomyocytes resulted in impaired cell survival upon inflammatory stimuli and the spontaneous development of fibrosis and cardiac dysfunction during the process of aging (Jacoby et al., 2003). These effects are partially attributed to its transcriptional pro-survival and anti-ROS actions. In recent years, some non-canonical interactions of STAT3 with the NF- $\kappa \mathrm{B}$ signalling pathway and with complex I of the mitochondrial electron-transport chain have been identified. These interactions have been shown to further reduce damage to cardiomyocytes by limiting damage by reactive oxygen species (ROS) (Zouein et al., 2015). Therefore, the current notion is that, in cardiomyocytes, STAT1 increases the cell damage by promoting apoptosis while STAT3 mediates pro-survival signalling.

The human heart consists of approximately four to five billion cardiomyocytes (Frangogiannis, 2012; Bonaventura et al., 2016), a cell type which has a very limited 
regenerative capacity. Upon myocardial infarction, functional cardiomyocytes are lost due to necrotic cell death as well as apoptosis. Tissue inflammation has been a main feature of ischemic injury of the heart and induces a pro-apoptotic environment often with damaging effects to the post-infarction myocardium. If a substantial amount of cardiomyocytes is lost, only defective healing marked by collagen deposition and scar formation but no functional healing by cardiomyocyte proliferation occurs (Scarabelli and Gottlieb, 2004; Frangogiannis, 2012; Bonaventura et al., 2016). Post-infarction cardiac repair is orchestrated in three phases. The first phase is characterised by massive invasion of the cardiac muscle by inflammatory cells. This leads to the clearance of the infarcted tissue of cell debris and dead cells and initiates reparative signalling pathways. During the second, proliferative phase, vascular cells and myofibroblasts are recruited by growth factors mainly secreted by macrophages. As a result, the structural integrity of the heart becomes restored by the synthesis of extracellular matrix proteins. During the third and final phase, many of the effector cells become quiescent or undergo apoptosis to avoid excess extracellular matrix deposition. The newly formed collagen is crosslinked to ensure structural integrity therefore completing the scar (Frangogiannis, 2012; Bonaventura et al., 2016).

Upon necrosis of the myocardium, damage-associated molecular patterns induce an activation of the innate immune system (Kono and Rock, 2008). The induction of damageassociated signalling was shown for several factors released during tissue necrosis, such as high mobility group box 1 (HMGB1), galectin-9 and heat-shock proteins. These factors induced maturation of dendritic cells, cytokine production, differentiation of Th1 cells and induction of receptor of advanced glycation end products (RAGE) signalling (Basu, 2000; Scaffidi et al., 2002; Dai et al., 2005). This pro-inflammatory environment leads to the recruitment of immune cells to the ischemic heart. The main effectors of this inflammatory response were identified as being neutrophil granulocytes, monocytes/macrophages, lymphocytes, mast cells, myofibroblasts, platelets and vascular cells (Bonaventura et al., 2016). Platelets are the first cells to become activated and localize to the infarcted area. Upon binding to adhesion or matrix proteins, they become activated and release a variety of chemotactic factors (Gawaz 2004). Binding of neutrophils with cardiomyocytes induced the intracellular generation of reactive oxygen species in both interaction partners (Entman et al., 1992). Adhesion molecules have received some attention in the context of the recruitment of inflammatory cells to the myocardium. Neutralizing antibodies against CD11a, CD11c, CD18, ICAM-1 and P-selectin were shown to reduce the infiltrate as well as the infarction size (Yamazaki et al., 1993; Tojo et al., 1996). However, first clinical trials translating the 
promising findings from cell-adhesion inhibition in animal models into humans by administration of neutralizing antibodies against integrins to patients during the first hours after myocardial infarction did not result in detectable beneficial effects (Christia and Frangogiannis, 2013).

Since the role of STAT1 upon myocardial ischemia has mainly be assessed in ex vivo models, in this project an in vivo model was applied to evaluate how STAT1-dependent signalling is involved in myocardial infarction and post-infarction remodelling. Ligation of the left anterior descending artery was performed on mice carrying the F77A knockin mutation in the Stat1 gene. During the first $96 \mathrm{~h}$ after the induction of myocardial infarction, no differences between wildtype and knockin mice were observed with respect to weight loss or sickness behaviour. Mortality during the first 4 days after infarction was below $10 \%$. However, between day 4 and day 7, survival of wildtype mice dropped from $87 \%$ to $52 \%$, while survival rates of the STAT1-F77A knockin mice decreased from $95 \%$ to $77 \%$ (Figure 19). In the study cohort, this effect was almost completely attributed to the male sex, while mortality rates of female mice were equally low in the two genotypes. In the male mice, the carriers of the STAT1-F77A mutant were protected from death during the follow-up period after myocardial infarction. The timing of the marked drop in survival, which is usually more pronounced in males than in females, is due to left ventricular rupture or heart failure - a phenomenon that has been reported in several studies to occur between the $2^{\text {nd }}$ and $7^{\text {th }}$ day after infarction (Scherrer-Crosbie et al., 2001; Gao et al., 2005; Xiang et al., 2009; Sauvé et al., 2010). A mouse model of ischemia/reperfusion injury in mice deficient for the IFN $\gamma$ induced gene $C c l 2$ resulted in attenuated and delayed cardiac remodelling which was caused by reduced recruitment of macrophages and myofibroblasts (Dewald et al., 2005). Diminished recruitment of macrophages to sites of inflammation was also described for a genetic knockout model of the gene encoding the CCL2 receptor (Kurihara et al., 1997). Since the STAT1-F77A mutant does not induce transcription of the CCL2 gene as efficiently as the wildtype (Figure 29), a reduced recruitment of macrophages to the infarcted hearts of the knockin mouse was expected. Another cytokine, which is usually induced via IFN $\gamma$-dependent STAT1 signalling, is CXCL10. In the STAT1-F77A knockin model, cxcl10 is only induced by IFN $\alpha$, while IFN $\gamma$ fails to induce gene transcription (Begitt et al. 2014, Figure 29). Ischemia/reperfusion injury of the heart in the CXCL10-deficient mouse was characterized by increased numbers of neutrophils in the early acute phase 24-72 $\mathrm{h}$ post-infarction and enhanced density of macrophages in the late acute phase 3-7 days after the infarction. This was accompanied by an increased recruitment of myofibroblasts and an early dilative 
remodelling leading to poor cardiac function (Bujak et al., 2009). Since both cytokines are less inducible in the STAT1-F77A knockin mouse model and one has proven beneficial while the other mediates adverse effects during post-infarction remodelling, the outcome of myocardial infarction in the STAT1-F77A mouse was difficult to predict. However, since both cytokines mediate the recruitment of different cellular subsets to the inflamed region, an overall reduced recruitment of immune cells resulting in attenuated post-infarction remodelling was expected.

Ligation of the left anterior descending artery was rapidly followed by massive infiltration of the myocardium with several leukocytes subsets. The extent of this infiltration did not differ among the wildtype and the homozygous STAT1-F77A knockin mouse line (Figure 20, Figure 21, Figure 27, and Figure 30-32) In the STAT1-F77A knockin mouse line, a higher number of cells staining positively for activated STAT1 was observed. This observation is in line with the in vitro data showing that the STAT1-F77A mutant displays prolonged and increased rates of phosphorylation compared with the wildtype molecule (Meyer et al., 2004). Cells stained positively for tyrosine-phosphorylated STAT1 were found at similar densities in the hearts of wildtype and knockin mice during the acute phase of myocardial infarction, although, in the transgenic mouse line, these cells were distributed over a larger area. The cells recruited during the acute phase are mainly neutrophils, monocytes/macrophages, and T cells, as demonstrated by means of immunohistochemistry. Massive infiltration of the myocardium with neutrophils was detected during the acute phase as well as a moderate infiltration with macrophages and T cells (Figure 30, Figure 31, and Figure 32). Neutrophil infiltration was pronounced in all genotypes and was significantly reduced in heterozygous mice, but not in homozygous mice carrying the STAT1-F77A mutation as compared to their wildtype littermates.

Protein lysates of the hearts revealed increased expression levels of STAT1 and STAT3 in the infarcted areas compared with lysates obtained from non-infarcted areas (Figure 26). While phosphorylation levels of STAT1 appeared to be fairly equal among the genotypes, there was a higher expression of STAT1 in the infarcted myocardium of wildtype mice as compared with the hetero- and homozygous STAT1-F77A knockin mice. Since the STAT1F77A mutant fails to activate STAT1-driven IFN $\gamma$ target genes, STAT3-mediated signalling may be preferred in this mouse line. Corroborating this hypothesis, it was found that the ratio of STAT3 to STAT1 expression is increased in homozygous STAT1-F77A mice as compared 
to wildtype and heterozygous animals, while the corresponding ratios of their phosphorylated forms were similar among the three genotypes.

Clinical studies in humans after myocardial infarction have identified ejection fraction, end-systolic volume and end-diastolic volume to be valid and strong predictors of postinfarction survival. Elevated end-systolic volume proved to be the most accurate predictor for post-infarction death from cardiac dysfunction-related causes (White et al., 1987). To some extent, post-infarction cardiac remodelling and scar formation can be seen as beneficial since it helps to restore and maintain cardiac output. Continued maladaptive cardiac remodelling, however, was linked to the progression of heart failure (Cohn et al., 2000).

When analysing for the effects of the STAT1-F77A knockin on post-infarction cardiac remodelling by means of echocardiography, the transgenic mouse line showed attenuated cardiac dilation in females (Figure 22-25). Analysis of the heterozygous genotype before and three days after myocardial infarction did not contradict the observed effect but was not identified clearly as showing a gene-dose effect or mimicking the results of the wildtype or the mutant (data not shown). The striking difference among the sexes was unexpected but could be attributed to the reduction in cohort size due to the high mortality rate during the first week after surgery. It is possible that, preferentially those animals of the study cohort which had the most severely affected heart function died. Corroborating the data from the pure anatomical measurements including the end-systolic and end-diastolic volume, hearts of STAT1-F77A mice showed an ameliorated cardiac performance during the course of cardiac remodelling (Figure 25). After MI injury, fractional area shortening, fractional shortening and ejection fraction were reduced in both wildtype and homozygous mutant mice. However, the drop was less pronounced in the IFN $\gamma$-irresponsive STAT1-F77A mutant.

All findings were most pronounced at week 1 after MI injury and had partially faded to being non-significant when the data obtained 4 weeks after the insult were analysed. Since only few female mice died during the follow-up, this result might indicate that the functional effect is transient. In the CCL2 knockout mouse, cardiac remodelling was delayed which resulted in a more favourable outcome with regard to cardiac performance (Dewald et al., 2005). Since CCL2 induction by the STAT1-F77A mutant is impaired, the observed effect could be attributed to a similar one mediated by fewer macrophages and a lower recruitment of myofibroblasts. However, this hypothesis must remain controversial since no reduction in macrophage recruitment was observed for the knockin mice in the early phase after myocardial injury. The reduced numbers of neutrophils in the myocardial infiltrates in 
heterozygous mice suggest that these animals may be protected from neutrophil-induced ROS generation (Entman et al., 1992). This favours higher rates of cardiomyocyte survival and improved cardiac function as a permanent effect. Although the exact mechanism remains to be identified, the data clearly show beneficial effects of impaired IFN $\gamma$-dependent signalling during the early phase after MI injury.

Cellular effects in myocardial infarction are the consequence of hypoxia-induced organ damage and are mainly mediated by processes of innate immunity including the recruitment of neutrophils and macrophages and the induction of extracellular matrix deposition (Bonaventura et al., 2016). In contrast, autoimmunity is a process resulting from the recognition of self-antigens by $\mathrm{B}$ or $\mathrm{T}$ lymphocytes. STAT1 and STAT3 and their downstream signalling effectors, have been attributed some functions during the development of several autoimmune diseases including multiple sclerosis (MS) and systemic lupus erythematosus (Feng et al., 2002; Ding et al., 2016). Therefore, the study was aimed at evaluating the clinical consequences of interactions between the innate immunity as activated by LPS challenge and factors of the adaptive immunity as induced by experimental autoimmune encephalomyelitis.

In this study, mice were injected with $5 \mathrm{mg} / \mathrm{kg}$ body weight LPS during the induction phase of EAE. This injection resulted in the development of severe symptoms of systemic inflammation and was accompanied by weight loss and general signs of fatigue. Interestingly, mortality was particularly high in the group of LPS-injected wildtype mice which express all components of intact IFN $\gamma$-dependent signalling (Figure 33). The results showed that the STAT1 genotype is a strong predictor of mortality in the sense that the STAT1 knockout mice are protected upon LPS treatment. This is in line with a report that mice deficient for STAT1, STAT4 or TYK2 are protected from LPS-induced sepsis-related death (Kamezaki et al., 2004).

Lipopolysaccharide is a bacterial substance that induces signalling via the toll-like receptor 4. The toll-like receptors (TLRs) are a class of pattern recognition receptors and part of the innate immune system which poses a first line defence mechanism in the recognition of characteristic structures displayed by microbial pathogens (Kawai and Akira, 2010). Although most studies on TLR4 have focused on its role in innate immunity, LPS-dependent TLR4 signalling was identified as promoter of the development of Th17 cells but not Th1 or Th2 cells (Park et al., 2015). 
Besides the direct effect of LPS in acute inflammation, mice injected with it displayed their first symptoms of EAE later than mice not co-treated with it (Figure 35). Wildtype mice that had received an LPS injection prior to disease development showed a higher variability in their disease onset with a mean delay of approximately one week. This effect is in line with a report of a delay of 5 days in EAE onset in mice in response to LPS treatment three days prior to EAE induction (Buenafe and Bourdette, 2007). In this study, the delay in onset was attributed to the induction of TNF $\alpha$, TGF $\beta$, IFN $\beta$ and SOCS3 in response to the TLR4 stimulus. This delay could also be reported for the STAT1-deficient mice but with a more consistent delay in disease onset of 1 week. The knockout mice developed slightly more pronounced disease symptoms regardless of whether they had been treated with LPS or not (Figure 34). However, this exacerbated disease progression was not as pronounced as had been reported earlier by the laboratory group of Bettelli (Bettelli et al., 2004). Most studies indicate protective and beneficial effects due to LPS treatment (e.g. Raziuddin and Morrison, 1982; Buenafe and Bourdette, 2007; Ellestad et al., 2009; Li et al., 2010), whereas there are some which name detrimental effects of LPS (e.g. Nogai et al., 2005). However, the protocols used in these studies vary widely with respect to the dose as well as the time point of LPS administration. In general, gavage of LPS to mice prior to the induction of EAE delayed or attenuated disease development.

One proposed mechanism of LPS action was the induction of IL-10 production by dendritic cells which has proven to be inhibitory to disease progression (Zhou et al., 2014). An earlier study showed that mice which produce transgenic IL-10 under the control of the $H 2$ promoter are protected from EAE induction (Cua et al., 1999). However, this notion might be problematic since LPS-induced IL-10 production is, at least in part, considered to result from type I interferon signalling (Iyer et al., 2010). High IL-10 production has been reported for the STAT1 knockout as well as the T-bet-STAT1 double knockout mice (Bettelli et al., 2004).

Experimental autoimmune encephalomyelitis is an inducible model of a neurodegenerative disease mimicking the main features of multiple sclerosis (MS). Most commonly, EAE is induced in rodent models such as mouse and rat but also protocols for EAE induction in nonhuman primates are utilised (Hart et al., 2015). Multiple sclerosis is among the most common causes of neurological disability in young adults with higher prevalence in females than in males. Owing to demyelination and axon loss, patients develop physical and mental disability. Approximately 2 million people are estimated to be affected by this disease worldwide, 
roughly half of whom live in Europe (Kingwell et al., 2013; Grigoriadis and van Pesch, 2015). According to disease progression, MS is categorized as the relapsing-remitting MS, which can develop into the secondary progressive form, or the primary progressive form of MS. Patients suffering from relapsing-remitting MS alternate between periods of severe disease symptoms and periods of almost complete recovery. In some cases, the disease course develops into the secondary progressive form with no recovery. Patients suffering from the primary progressive form do not go into remission (Kieseier and Hartung, 2003).

On the immunological level, MS is a disease mediated by dysfunction of parts of the innate, as well as, the adaptive immune system. Infiltration of the CNS, mainly the white matter by Th1 and Th17 cells, is a characteristic feature of the disease. Release of inflammatory cytokines activates macrophages and microglia which leads to the production of reactive oxygen and nitrogen species. In this pro-inflammatory environment, axons are demyelinated and degenerate leading to neurological disease symptoms (Grigoriadis and van Pesch, 2015). In the model of $\mathrm{MOG}_{35-55}$ peptide-induced EAE, dendritic cells pick up the peptide, migrate to the lymph nodes and cross-present the antigen to naïve $\mathrm{T}$ cells (Slavin et al., 2001). Symptoms are caused by the demyelination of axons of CNS neurons evoked by invasion of the CNS by autoreactive T cells, and the onset of disease is preceded by the activation and proliferation of T cells in the lymphatic organs (Soulika et al., 2009).

Several studies in mice have reported that impairment of the IFN $\gamma$-STAT1 axis, including genetic knockout of the genes encoding for STAT1, IFN $\gamma$ or the ligand-binding chain of IFNGR, do not ameliorate EAE development and progression but rather augments disease severity, susceptibility and mortality (Ferber et al., 1996; Krakowski and Owens, 1996; Willenborg et al., 1996; Bettelli et al., 2004). Differences in numbers and function of regulatory $\mathrm{T}$ cells have been suggested as an underlying mechanism for the EAE progression in these transgenic models. In $129 \mathrm{~Sv}$ mice, reduced numbers of regulatory $\mathrm{T}$ cells, as defined by the co-expression of CD4 and CD25, have been reported in STAT1-deficient mice as compared to a wildtype background (Nishibori et al., 2004). Furthermore, STAT1-deficient regulatory $\mathrm{T}$ cells were reported to lack the ability to inhibit growth. This observation contradicts our analysis of decreased numbers of regulatory $\mathrm{T}$ cells in the IFN $\gamma$-irresponsive STAT1 knockin mouse line, as shown in Figure 28. Some studies have reported that FoxP3positive induced regulatory T (iTreg) cells are less frequent in STAT1- and in IRF1-deficient mice and that IFN $\gamma$-signalling is either a suppressor or inducer of Treg development (Wang et al., 2006; Fragale et al., 2008; Chang et al., 2009). The results from the experiments with the 
STAT1-F77A knockin mouse line support studies showing that defective IFN $\gamma$ signaling is linked to increased iTreg differentiation.

A different mechanism to explain the protective effect of IFN $\gamma$-dependent signalling in EAE was the induction of iNOS which leads to increased levels of reactive nitrogen species (Willenborg et al., 1999). The inhibitory effect of increased NO production resulting from IFN $\gamma$ - or concanavalin A-induced signalling has been repeatedly reported (Albina et al., 1991; Krenger et al., 1996; Zettl et al., 1997). This mechanism was identified as specific for Th1 activation: IFN $\gamma$ which was released from Th1 cells inhibited exacerbated Th1 activation resulting from NO production by APCs (van der Veen et al., 2000). Deficiency in iNOS expression was shown to hinder remission during the course of EAE. Overall, lack of iNOS was shown to augment EAE progression in mice (Fenyk-Melody et al., 1998; Sahrbacher et al., 1998). Furthermore, IFN $\gamma$-dependent signalling has been seen to regulate activationinduced cell death in T lymphocytes (Refaeli et al., 2002).

In an adoptive transfer model of EAE, Th1 recipient mice displayed a chemokine profile of high CXCL9, CXCL10 and CXCL11 expression, while Th17 recipients secreted CXCL1 and CXCL2. Although the cytokine profiles were distinct, clinical disease progression was similar in Th1- and Th17-mediated disease (Kroenke et al., 2008). In IFN $\gamma$ knockout mice, the recruitment of inflammatory cells to the CNS is mainly mediated by CXCL2 and CCL1, not by CCL5 and CCL2 as in the wildtype (Tran et al., 2000). In an experimental autoimmune uveitis model, a similar pattern of recruitment of immune cells via the Th2 and Th17 cytokines CCL1, CXCL2, CCL11, CCL17 and CCL22 instead of the Th1-dependent cytokines CCL5, CXCL9, and CXCL10 was observed for the IFN $\gamma$ knockout mouse (Su et al., 2007). Here, the differential expression of cytokines led to an increased recruitment of neutrophil and eosinophil granulocytes and a reduced recruitment of lymphocytes and monocytes.

In vitro proliferation assays using cell isolates derived from spleen and lymph nodes of $\mathrm{MOG}_{35-55}$ peptide-immunized mice shortly before the predicted onset of initial disease symptoms demonstrated a striking difference among the cells of wildtype and STAT1deficient mice (Figure 36). While $\mathrm{MOG}_{35-55}$ peptide and concanavalin A both readily induced proliferation in cells from wildtype lymph nodes, cells derived from STAT1 knockout mice showed an even stronger increase in proliferation in response to either stimulus. This observation is in line with the notion that disease symptoms were augmented by the STAT 1 knockout genotype as indicated by the severity of clinical symptoms. In support of these 
findings, T cells from STAT1 knockout mice have been reported to be hyperproliferative (Bettelli et al., 2004). Increased proliferation of activated $\mathrm{CD} 4^{+} \mathrm{T}$ cells characterised by high expression of CD44 and low expression of CD62L has been previously reported for IFN $\gamma$ deficient mice (Chu et al., 2000). In this model, the effect was, in part, attributed to failure of APCs to induce apoptosis.

Pre-treatment of mice with LPS showed little effect on the proliferation of cells of the wildtype genotype (Figure 36). However, the analysis of the impact of LPS on proliferation in the knockout genotype, revealed that the proliferation rate in response to $\mathrm{MOG}_{35-55}$ peptide was critically impaired while the response to concanavalin A appeared to be unchanged. These findings indicate that LPS has little effect on the induction of proliferation of T cells in spleen and lymph nodes of wildtype animals, whereas it impairs proliferation in cells from STAT1 knockout mice. MHC class II molecules are under transcriptional control of STAT1. A study using early life exposure of mice to LPS showed a protective effect of this treatment on EAE severity which was mainly attributed to reduced expression of MHC class II molecules on antigen presenting cells (Ellestad et al., 2009). Since it had been shown that STAT1 signalling in APCs limits T cell proliferation, the observations can be explained by an impaired antigen presentation after LPS treatment with an overall impaired effect on growth inhibition in the STAT1 knockout (van der Veen et al., 2000).

Reports on the mechanisms underlying the onset and progression of EAE have attributed major roles to $\mathrm{CD}^{+}$lymphocytes and, in particular, Th1 and Th17 cells (Rangachari and Kuchroo, 2013; Bonelli et al., 2014). Th1 and Th17 cells are characterised by the secretion of marker cytokines which are IFN $\gamma$ in the case of Th1 cells and IL-17A in Th17 cells (Mosmann et al., 1986; Park et al., 2005). Assessment of the release of these cytokines in supernatants of ex vivo cultures of spleen and lymph node cells led to the notion that cells from immunized STAT1-deficient mice readily released large amounts of both cytokines in response to $\mathrm{MOG}_{35-55}$ peptide encounter (Figure 37 and Figure 38). This observation was surprising since IFN $\gamma$-dependent signalling is mainly transduced via the transcription factor STAT1, and the gene encoding T-bet, TBX21, is a known STAT1-target gene and the masterregulator of Th1 differentiation (Lighvani et al., 2001; Afkarian et al., 2002; Villarino et al., 2010). The expectation in this context was that the Th17 lineage would be preferred in the absence of IFN $\gamma / \mathrm{STAT} 1-d e p e n d e n t$ signal transduction (Harrington et al., 2005). Furthermore, it has been reported that lack of STAT1-mediated signalling usually pushes a STAT3-dependent reaction with STAT3 as a regulator of ROR $\gamma \mathrm{t}$, the main inducer of Th17 
maturation. Disturbances of the balance between STAT1- and STAT3-dependent signal pathways have been linked to altered lineage decision as a response to typical Th1 or Th17 inducing cytokines (Qing and Stark, 2004; Yang et al., 2007; Peters et al., 2015; Wan et al., 2015). However, although T-bet is regulated by IFN $\gamma$-dependent STAT1 signalling, it can be upregulated in $\mathrm{T}$ cell subsets in the absence of IFN $\gamma$ signalling and even in the combined knockdown of IFN $\gamma$ and IL-12. In the context of T cell fate, IFN $\gamma$-producing and IL-17Aproducing T cells developed as well as T cells expressing both cytokines (Yeh et al., 2011). A study on Th17 cells showed that commitment towards this lineage was not permanent. Upon treatment of Th17 cells with Th1- or Th2-inducing factors, the production of Th1 and Th2 marker cytokines could still be induced (Stritesky et al., 2008). IL-23 has been identified as an agent which pushes the development of IL-17A and IFN $\gamma$ double positive cells from Th17 cells. Knockout of STAT1, STAT4 or TBX21 did not reduce the size of this T cell population (Duhen et al., 2013). Similar to its effect in leukocyte proliferation, LPS treatment abrogated the release of IL-17A and IFN $\gamma$ by the cultured cells. This effect was similar in the two genotypes and in line with the observation of delayed onset of disease symptoms after treatment with LPS. Therefore, the strong induction of cytokine production in the STAT1 knockout can, in part, be explained by the strong proliferation. Additionally, Th17 cells that acquire the double positive phenotype of IL-17A and IFN $\gamma$-producing cells can also contribute to the high secretion of both cytokines.

In summary, this project underscores the protomer exchange kinetics as a critical step in the STAT1 activation/inactivation cycle and describes this process as an alternative mechanism for the conformational change between the parallel and anti-parallel dimer conformation. Furthermore, it was shown that DNA binding of STAT1 not only hinders dephosphorylation but also the exchange of STAT1 protomers. The gene encoding the cytoskeletal protein ezrin was identified as a STAT1-regulated target gene since binding of tyrosine-phosphorylated STAT1 to the EZR promoter induced a weak transcriptional response. This effect could not be found at the level of protein expression which might indicate a counteracting mechanism. The phenotype of the STAT1-F77A mutant was further characterised by showing that this mutant was proficient in facilitating normal monomer exchange kinetics. Moreover, this mutant, similar to the complete functional knockout of STAT1, displayed no reduced expression levels of ezrin or moesin, supporting the notion that STAT1 is dispensable for the baseline expression of the two ERM proteins. In the context of inflammation following myocardial infarction, STAT1-F77A knockin males showed significantly higher rates of survival, while females were partially protected from cardiac 
dilation and had a less impaired cardiac function when compared with their wildtype littermates. Analysis of the inflammatory infiltrate revealed a minor increase in cells expressing activated STAT1, while slightly fewer neutrophil granulocytes were recruited during the acute phase in heterozygous STAT1-F77A knockin mice. Analysis of the role of STAT1 in autoimmunity using EAE as the model of choice, revealed hyperproliferation and increased production of Th1 and Th17 marker cytokines in the STAT1 knockout context. These findings emphasize the role of STAT1 in non-infectious inflammatory diseases. 


\section{References}

Abroun S, Saki N, Ahmadvand M, Asghari F, Salari F, Rahim F. STATs: An old story, yet mesmerizing. Cell J 2015;17(3):395-411.

Afkarian M, Sedy JR, Yang J, Jacobson NG, Cereb N, Yang SY, Murphy TL, Murphy KM. T-bet is a STAT1-induced regulator of IL-12R expression in naïve CD4+ $\mathrm{T}$ cells. Nat Immunol 2002;3(6):549-57.

Albina JE, Abate JA, Henry WL Jr. Nitric oxide production is required for murine resident peritoneal macrophages to suppress mitogen-stimulated T cell proliferation. Role of IFN-gamma in the induction of the nitric oxide-synthesizing pathway. J Immunol 1991;147(1):144-8.

Alexander WS. Suppressors of cytokine signalling (SOCS) in the immune system. Nat Rev Immunol 2002;2(6):410-6.

Alonzi T, Maritano D, Gorgoni B, Rizzuto G, Libert C, Poli V. Essential role of STAT3 in the control of the acute-phase response as revealed by inducible gene inactivation in the liver. Mol Cell Biol 2001;21(5):1621-32.

Bach EA, Aguet M, Schreiber RD. The IFN gamma receptor: a paradigm for cytokine receptor signaling. Annu Rev Immunol 1997;15:563-91.

Baden HA, Sarma SP, Kapust RB, Byrd RA, Waugh DS. The amino-terminal domain of human STAT4. Overproduction, purification, and biophysical characterization. $J$ Biol Chem 1998;273(27):17109-14.

Bagawath-Singh S, Staaf E, Stoppelenburg AJ, Spielmann T, Kambayashi T, Widengren J, Johansson S. Cytokines induce faster membrane diffusion of MHC class I and the Ly49A receptor in a subpopulation of natural killer cells. Front Immunol 2016;7:16.

Barreiro O, Yanez-Mo M, Serrador JM, Montoya MC, Vicente-Manzanares M, Tejedor R, Futhmayr H, Sanchez-Madrid F. Dynamic interaction of VCAM-1 and ICAM-1 with moesin and ezrin in a novel endothelial docking structure for adherent leukocytes. J Cell Biol 2002;157(7):1233-45.

Basu S, Binder RJ, Suto R, Anderson KM, Srivastava PK. Necrotic but not apoptotic cell death releases heat shock proteins, which deliver a partial maturation signal to dendritic cells and activate the NF-kappaB pathway. Int Immunol 2000;12(11):1539-46.

Becker S, Groner B, Müller CW. Three-dimensional structure of the Stat3beta homodimer bound to DNA. Nature 1998;394(6689):145-51.

Begitt A, Droescher M, Meyer T, Schmid CD, Baker M, Antunes F, Knobeloch KP, Owen MR, Naumann R, Decker T, Vinkemeier U. STAT1-cooperative DNA binding distinguishes type 1 from type 2 interferon signaling. Nat immunol 2014;15(2):168-76.

Begitt A, Meyer T, van Rossum M, Vinkemeier U. Nucleocytoplasmic translocation of Stat1 is regulated by a leucine-rich export signal in the coiled-coil domain. Proc Natl Acad Sci U S A 2000;97(19):10418-23.

Bettelli E, Carrier Y, Gao W, Korn T, Strom TB, Oukka M, Weiner HL, Kuchroo VK. Reciprocal developmental pathways for the generation of pathogenic effector TH17 and regulatory T cells. Nature 2006;441(7090):235-8. 
Bettelli E, Sullivan B, Szabo SJ, Sobel RA, Glimcher LH, Kuchroo VK. Loss of T-bet, but not STAT1, prevents the development of experimental autoimmune encephalomyelitis. $J$ Exp Med 2004;200(1):79-87.

Boengler K, Hilfiker-Kleiner D, Drexler H, Heusch G, Schulz R. The myocardial JAK/STAT pathway: from protection to failure. Pharmacol Ther 2008;120(2):172-85.

Bonaventura A, Montecucco F, Dallegri F. Cellular recruitment in myocardial ischaemia/reperfusion injury. Eur J Clin Invest 2016;46(6):590-601.

Bonelli M, Shih H, Hirahara K, Singelton K, Laurence A, Poholek A, Hand T, Mikami Y, Vahedi G, Kanno Y, O'Shea JJ. Helper $\mathrm{T}$ cell plasticity: impact of extrinsic and intrinsic signals on transcriptomes and epigenomes. Curr Top Microbiol Immunol 2014;381:279-326.

van Boxel-Dezaire AH, Zula JA, Xu Y, Ransohoff RM, Jacobberger JW, Stark GR. Major differences in the responses of primary human leukocyte subsets to IFN-beta. J Immunol 2010;185(10):588899.

Bradford MM. A rapid and sensitive method for the quantitation of microgram quantities of protein utilizing the principle of protein-dye binding. Anal Biochem 1976;72:248-54.

Bretscher A, Edwards K, Fehon RG. ERM proteins and merlin: integrators at the cell cortex. Nat Rev Mol Cell Biol 2002;3(8):586-99.

Buenafe AC, Bourdette DN. Lipopolysaccharide pretreatment modulates the disease course in experimental autoimmune encephalomyelitis. J Neuroimmunol 2007;182(1-2):32-40.

Bujak M, Dobaczewski M, Gonzalez-Quesada C, Xia Y, Leucker T, Zymek P, Veeranna V, Tager $\mathrm{AM}$, Luster AD, Frangogiannis NG. Induction of the CXC chemokine interferon-gamma-inducible protein 10 regulates the reparative response following myocardial infarction. Circ Res 2009;105(10):973-83.

Carbognin E, Betto RM, Soriano ME, Smith AG, Martello G. Stat3 promotes mitochondrial transcription and oxidative respiration during maintenance and induction of naïve pluripotency. EMBO J 2016;35(6):618-34

Caretto D, Katzman SD, Villarino AV, Gallo E, Abbas AK. Cutting edge: the Th1 response inhibits the generation of peripheral regulatory T cells. J Immunol 2010;184(1):30-4.

Cernuda-Morollón E, Millán J, Shipman M, Marelli-Berg FM, Ridley AJ. Rac activation by the T-cell receptor inhibits T cell migration. PloS One 2010;5(8):e12393.

Cetin B, Gonul II, Buyukberber S, Afsar B, Gumusay O, Algin E, Turan N, Ozet A, Benekli M, Coskun U. The impact of immunohistochemical staining with ezrin-carbonic anhydrase IX and neuropilin-2 on prognosis in patients with metastatic renal cell cancer receiving tyrosine kinase inhibitors. Tumour Biol 2015;36(11):8471-8.

Chang JH, Kim YJ, Han SH, Kang CY. IFN-gamma-STAT1 signal regulates the differentiation of inducible Treg: potential role for ROS-mediated apoptosis. Eur J Immunol 2009;39(5):1241-51.

Chauhan AK, Moore TL. T cell activation by terminal complex of complement and immune complexes. J Biol Chem 2011;286(44):38627-37.

Cheeran MC, Mutnal MB, Hu S, Armien A, Lokensgard JR. Reduced lymphocyte infiltration during cytomegalovirus brain infection of interleukin-10-deficient mice. J Neurovirol 2009;15(4):334-42. 
Chen EJ, Shaffer MH, Williamson EK, Huang Y, Burkhardt JK. Ezrin and moesin are required for efficient T cell adhesion and homing to lymphoid organs. PloS One 2013;8(2):e52368.

Chen W, Jin W, Hardegen N, Lei KJ, Li L, Marinos N, McGrady G, Wahl SM. Conversion of peripheral CD4+CD25- naive $\mathrm{T}$ cells to $\mathrm{CD} 4+\mathrm{CD} 25+$ regulatory $\mathrm{T}$ cells by TGF-beta induction of transcription factor Foxp3. J Exp Med 2003a;198(12):1875-86.

Chen X, Bhandari R, Vinkemeier U, van Den Akker F, Darnell JE Jr, Kuriyan J. A reinterpretation of the dimerization interface of the N-terminal domains of STATs. Protein Sci 2003b;12(2):361-5.

Chen X, Vinkemeier U, Zhao Y, Jeruzalmi D, Darnell JE Jr, Kuriyan J. Crystal structure of a tyrosine phosphorylated STAT-1 dimer bound to DNA. Cell 1998;93(5):827-39.

Cheon H, Stark GR. Unphosphorylated STAT1 prolongs the expression of interferon-induced immune regulatory genes. Proc Natl Acad Sci U S A 2009;106(23):9373-8.

Christia P, Frangogiannis NG. Targeting inflammatory pathways in myocardial infarction. Eur J Clin Invest 2013;43(9):986-95.

Chu CQ, Wittmer S, Dalton DK. Failure to suppress the expansion of the activated CD4 T cell population in interferon gamma-deficient mice leads to exacerbation of experimental autoimmune encephalomyelitis. J Exp Med 2000;192(1):123-8.

Cohen SN, Chang AC, Hsu L. Nonchromosomal antibiotic resistance in bacteria: genetic transformation of Escherichia coli by R-factor DNA. Proc Natl Acad Sci U S A 1972;69(8):21104.

Cohn JN, Ferrari R, Sharpe N. Cardiac remodeling — concepts and clinical implications: A consensus paper from an international forum on cardiac remodeling. J Am Coll Cardiol 2000;35(3):569-82.

Collins SJ, Gallo RC, Gallagher RE. Continuous growth and differentiation of human myeloid leukaemic cells in suspension culture. Nature 1977;270(5635):347-9.

Copeland NG, Gilbert DJ, Schindler C, Zhong Z, Wen Z, Darnell JE Jr, Mui AL, Miyajima A, Quelle FW, Ihle JN, Jenkins NA. Distribution of the mammalian Stat gene family in mouse chromosomes. Genomics 1995(29):225-8.

Cross AK, Woodroofe MN. Chemokines induce migration and changes in actin polymerization in adult rat brain microglia and a human fetal microglial cell line in vitro. J Neurosci Res 1999;55(1):17-23.

Cua DJ, Groux H, Hinton DR, Stohlman SA, Coffman RL. Transgenic interleukin 10 prevents induction of experimental autoimmune encephalomyelitis. J Exp Med 1999;189(6):1005-10.

Cypowyj S, Picard C, Maródi L, Casanova J, Puel A. Immunity to infection in IL-17-deficient mice and humans. Eur J Immunol 2012;42(9):2246-54.

Dai S, Nakagawa R, Itoh A, Murakami H, Kashio Y, Abe H, Katoh S, Kontani K, Kihara M, Zhang SL, Hata T, Nakamura T, Yamauchi A, Hirashima M. Galectin-9 induces maturation of human monocyte-derived dendritic cells. J Immunol 2005;175(5):2974-81.

Darnell JE Jr. STATs and gene regulation. Science 1997;277(5332):1630-5.

Darnell JE Jr, Kerr IM, Stark GR. Jak-STAT pathways and transcriptional activation in response to IFNs and other extracellular signaling proteins. Science 1994;264(5164):1415-21.

Decker T, Kovarik P. Serine phosphorylation of STATs. Oncogene 2000;19(21):2628-37. 
Decker T, Kovarik P, Meinke A. GAS elements: a few nucleotides with a major impact on cytokineinduced gene expression. J Interferon Cytokine Res 1997;17(3):121-34.

Denker SP, Barber DL. Cell migration requires both ion translocation and cytoskeletal anchoring by the Na-H exchanger NHE1. J Cell Biol 2002;159(6):1087-96.

Deshpande RV, Goust JM, Chakrabarti AK, Barbosa E, Hogan EL, Banik NL. Calpain expression in lymphoid cells. Increased mRNA and protein levels after cell activation. J Biol Chem 1995;270(6):2497-505.

Devaux P, Priniski L, Cattaneo R. The measles virus phosphoprotein interacts with the linker domain of STAT1. Virology 2013;444(1-2):250-6.

Dewald O, Zymek P, Winkelmann K, Koerting A, Ren G, Abou-Khamis T, Michael LH, Rollins BJ, Entmann ML, Frangogiannis NG. CCL2/monocyte chemoattractant protein-1 regulates inflammatory responses critical to healing myocardial infarcts. Circ Res 2005;96(8):881-9.

Ding C, Chen X, Dascani P, Hu X, Bolli R, Zhang H, Mcleish KR, Yan J. STAT3 signaling in B cells is critical for germinal center maintenance and contributes to the pathogenesis of murine models of lupus. J Immunol 2016;196(11):4477-86.

Doi Y, Itoh M, Yonemura S, Ishihara S, Takano H, Noda T, Tsukita S. Normal development of mice and unimpaired cell adhesion/cell motility/actin-based cytoskeleton without compensatory upregulation of ezrin or radixin in moesin gene knockout. J Biol Chem 1999;274(4):2315-21.

Duhen R, Glatigny S, Arbelaez CA, Blair TC, Oukka M, Bettelli E. Cutting edge: the pathogenicity of IFN-gamma-producing Th17 cells is independent of T-bet. J Immunol 2013;190(9):4478-82.

Durbin JE, Hackenmiller R, Simon MC, Levy DE. Targeted disruption of the mouse Stat 1 gene results in compromised innate immunity to viral disease. Cell 1996;84(3):443-50.

Ellestad KK, Tsutsui S, Noorbakhsh F, Warren KG, Yong VW, Pittman QJ, Power C. Early life exposure to lipopolysaccharide suppresses experimental autoimmune encephalomyelitis by promoting tolerogenic dendritic cells and regulatory T cells. J Immunol 2009;183(1):298-309.

Elzagheid A, Korkeila E, Bendardaf R, Buhmeida A, Heikkilä S, Vaheri A, Syrjänen K, Pyrhönen S, Carpén O. Intense cytoplasmic ezrin immunoreactivity predicts poor survival in colorectal cancer. Hum Pathol 2008;39(12):1737-43.

Endo K, Kondo S, Shackleford J, Horikawa T, Kitagawa N, Yoshizaki T, Furukawa M, Zen Y, Pagano JS. Phosphorylated ezrin is associated with EBV latent membrane protein 1 in nasopharyngeal carcinoma and induces cell migration. Oncogene 2009;28(14):1725-35.

Entman ML, Youker K, Shoji T, Kukielka G, Shappell SB, Taylor AA, Smith CW. Neutrophil induced oxidative injury of cardiac myocytes. A compartmented system requiring CD11b/CD18ICAM-1 adherence. J Clin Invest 1992;90(4):1335-45.

Fagerlund R, Mélen K, Kinnunen L, Julkunen I. Arginine/lysine-rich nuclear localization signals mediate interactions between dimeric STATs and importin alpha 5. J Biol Chem 2002;277(33):30072-8.

Fehon RG, McClatchey AI, Bretscher A. Organizing the cell cortex: the role of ERM proteins. Nat Rev Mol Cell Biol 2010;11(4):276-87. 
Feng X, Petraglia AL, Chen M, Byskosh PV, Boos MD, Reder AT. Low expression of interferonstimulated genes in active multiple sclerosis is linked to subnormal phosphorylation of STAT1. J Neuroimmunol 2002;129(1-2):205-15.

Fenyk-Melody JE, Garrison AE, Brunnert SR, Weidner JR, Shen F, Shelton BA, Mudgett JS. Experimental autoimmune encephalomyelitis is exacerbated in mice lacking the NOS2 gene. J Immunol 1998;160(6):2940-6.

Ferber IA, Brocke S, Taylor-Edwards C, Ridgway W, Dinisco C, Steinman L, Dalton D, Fathman CG. Mice with a disrupted IFN-gamma gene are susceptible to the induction of experimental autoimmune encephalomyelitis (EAE). J Immunol 1996;156(1):5-7.

Fischer P, Hilfiker-Kleiner D. Role of gp130-mediated signalling pathways in the heart and its impact on potential therapeutic aspects. Br J Pharmacol 2008;153 Suppl 1:S414-27.

Fischer P, Hilfiker-Kleiner D. Survival pathways in hypertrophy and heart failure: the gp130-STAT axis. Basic Res Cardiol 2007;102(5):393-411.

Flaishon L, Lantner F, Hershkoviz R, Levo Y, Shachar I. Low levels of IFN-gamma down-regulate the integrin-dependent adhesion of $\mathrm{B}$ cells by activating a pathway that interferes with cytoskeleton rearrangement. J Biol Chem 2001;276(50):46701-6.

Fontenot JD, Rasmussen JP, Gavin MA, Rudensky AY. A function for interleukin 2 in Foxp3expressing regulatory T cells. Nat Immunol 2005;6(11):1142-51.

Fragale A, Gabriele L, Stellacci E, Borghi P, Perrotti E, Ilari R, Lanciotti A, Remoli AL, Venditti M, Belardelli F, Battistini A. IFN regulatory factor-1 negatively regulates CD4+ CD25+ regulatory T cell differentiation by repressing Foxp3 expression. J Immunol 2008;181(3):1673-82.

Frangogiannis NG. Regulation of the inflammatory response in cardiac repair. Circ Res 2012;110(1):159-73.

Frausto-Del-Río D, Soto-Cruz I, Garay-Canales C, Ambriz X, Soldevila G, Carretero-Ortega J, Vázquez-Prado J, Ortega E. Interferon gamma induces actin polymerization, Rac1 activation and down regulates phagocytosis in human monocytic cells. Cytokine 2012;57(1):158-68.

Frisullo G, Angelucci F, Caggiula M, Nociti V, Iorio R, Patanella AK, Sancricca C, Mirabella M, Tonali PA, Batocchi AP. pSTAT1, pSTAT3, and T-bet expression in peripheral blood mononuclear cells from relapsing-remitting multiple sclerosis patients correlates with disease activity. J Neurosci Res 2006;84(5):1027-36.

$\mathrm{Fu} \mathrm{XY}$. A transcription factor with $\mathrm{SH} 2$ and SH3 domains is directly activated by an interferon $\alpha$ induced cytoplasmic protein tyrosine kinase(s). Cell 1992;70(2):323-35.

Fuchs M, Hilfiker A, Kaminski K, Hilfiker-Kleiner D, Guener Z, Klein G, Podewski E Schieffer B, Rose-John S, Drexler H. Role of interleukin-6 for LV remodeling and survival after experimental myocardial infarction. FASEB J 2003;17(14):2118-20.

Furutani Y, Matsuno H, Kawasaki M, Sasaki T, Mori K, Yoshihara Y. Interaction between telencephalin and ERM family proteins mediates dendritic filopodia formation. $J$ Neurosci 2007;27(33):8866-76.

Galli A, Lombardi F. Postinfarct left ventricular remodelling: a prevailing cause of heart failure. Cardiol Res Pract 2016;2016:2579832. 
Gao XM, Xu Q, Kiriazis H, Dart AM, Du XJ. Mouse model of post-infarct ventricular rupture: time course, strain- and gender-dependency, tensile strength, and histopathology. Cardiovasc Res 2005;65(2):469-77.

Gawaz M. Role of platelets in coronary thrombosis and reperfusion of ischemic myocardium. Cardiovasc Res 2004;61(3):498-511.

Gillis S, Watson J. Biochemical and biological characterization of lymphocyte regulatory molecules. $\mathrm{V}$. Identification of an interleukin 2-producing human leukemia $\mathrm{T}$ cell line. J Exp Med 1980;152(6):1709-19.

Grigoriadis N, van Pesch V. A basic overview of multiple sclerosis immunopathology. Eur J Neurol 2015;22 Suppl 2:3-13.

Gutzmer R, Lisewski M, Zwirner J, Mommert S, Diesel C, Wittmann M, Kapp A, Werfel T. Human monocyte-derived dendritic cells are chemoattracted to $\mathrm{C} 3 \mathrm{a}$ after up-regulation of the C3a receptor with interferons. Immunology 2004;111(4):435-43.

Hamada K, Shimizu T, Matsui T, Tsukita S, Hakoshima T. Structural basis of the membrane-targeting and unmasking mechanisms of the radixin FERM domain. EMBO J 2000;19(17):4449-62.

Harada T, Kyttaris V, Li Y, Juang YT, Wang Y, Tsokos GC. Increased expression of STAT3 in SLE T cells contributes to enhanced chemokine-mediated cell migration. Autoimmunity 2007;40(1):1-8.

Harrington LE, Hatton RD, Mangan PR, Turner H, Murphy TL, Murphy KM, Weaver CT. Interleukin 17-producing CD4+ effector T cells develop via a lineage distinct from the Thelper type 1 and 2 lineages. Nat Immunol 2005;6(11):1123-32.

Hart BA 't, van Kooyk Y, Geurts JJ, Gran B. The primate autoimmune encephalomyelitis model; a bridge between mouse and man. Ann Clin Transl Neurol 2015;2(5):581-93.

Heusch G, Musiolik J, Gedik N, Skyschally A. Mitochondrial STAT3 activation and cardioprotection by ischemic postconditioning in pigs with regional myocardial ischemia/reperfusion. Circ Res 2011;109(11):1302-8.

Hilfiker-Kleiner D, Hilfiker A, Fuchs M, Kaminski K, Schaefer A, Schieffer B, Hillmer A, Schmiedl A, Ding Z, Podewski E, Podeswki E, Poli V, Schneier MD, Schulz R, Park JK, Wollert KC, Drexler H. Signal transducer and activator of transcription 3 is required for myocardial capillary growth, control of interstitial matrix deposition, and heart protection from ischemic injury. Circ Res 2004;95(2):187-95.

Hilfiker-Kleiner D, Shukla P, Klein G, Schaefer A, Stapel B, Hoch M, Müller W, Scherr M, Theilmeier G, Ernst M, Hilfiker A, Drexler H. Continuous glycoprotein-130-mediated signal transducer and activator of transcription-3 activation promotes inflammation, left ventricular rupture, and adverse outcome in subacute myocardial infaction. Circulation 2010;122(2):14-55.

Hoeve J ten, Jesus Ibarra-Sanchez M de, Fu Y, Zhu W, Tremblay M, David M, Shuai K. Identification of a nuclear Stat1 protein tyrosine phosphatase. Mol Cell Biol 2002;22(16):5662-8.

Horvath CM. STAT proteins and transcriptional responses to extracellular signals. Trends Biochem Sci 2000;25(10):496-502.

Hu X, Herrero C, Li W, Antoniv TT, Falck-Pedersen E, Koch AE, Woods JM, Haines GK, Ivashkiv LB. Sensitization of IFN-gamma Jak-STAT signaling during macrophage activation. Nat Immunol 2002;3(9):859-66. 
Hu X, Ivashkiv LB. Cross-regulation of signaling pathways by interferon-gamma: implications for immune responses and autoimmune diseases. Immunity 2009;31(4):539-50.

Hüntelmann B, Staab J, Herrmann-Lingen C, Meyer T. A conserved motif in the linker domain of STAT1 transcription factor is required for both recognition and release from high-affinity DNAbinding sites. PloS One 2014;9(5):e97633.

Ishihara S, Nishikimi A, Umemoto E, Miyasaka M, Saegusa M, Katagiri K. Dual functions of Rap1 are crucial for T-cell homeostasis and prevention of spontaneous colitis. Nat Commun 2015;6:8982.

Ivanov II, McKenzie BS, Zhou L, Tadokoro CE, Lepelley A, Lafaille JJ, Cua DJ, Littman DR. The orphan nuclear receptor RORgammat directs the differentiation program of proinflammatory IL17+ T helper cells. Cell 2006;126(6):1121-33.

Ivetic A, Ridley AJ. Ezrin/radixin/moesin proteins and Rho GTPase signalling in leucocytes. Immunology 2004;112(2):165-76.

Iyer SS, Ghaffari AA, Cheng G. Lipopolysaccharide-mediated IL-10 transcriptional regulation requires sequential induction of type I IFNs and IL-27 in macrophages. J Immunol 2010;185(11):6599-607.

Jacoby JJ, Kalinowski A, Liu M, Zhang SS, Gao Q, Chai G, Ji L, Iwamoto Y, Li E, Schneider M, Russell KS, Fu XY. Cardiomyocyte-restricted knockout of STAT3 results in higher sensitivity to inflammation, cardiac fibrosis, and heart failure with advanced age. Proc Natl Acad Sci U S A 2003;100(22):12929-34.

John S, Vinkemeier U, Soldaini E, Darnell JE Jr, Leonard WJ. The significance of tetramerization in promoter recruitment by Stat5. Mol Cell Biol 1999;19(3):1910-8.

Jouanguy E, Zhang S, Chapgier A, Sancho-Shimizu V, Puel A, Picard C, Boisson-Dupuis S, Abel L, Casanova JL. Human primary immunodeficiencies of type I interferons. Biochimie 2007;89(67):878-83.

Kamezaki K, Shimoda K, Numata A, Matsuda T, Nakayama KI, Harada M. The role of Tyk2, Stat1 and Stat4 in LPS-induced endotoxin signals. Int Immunol 2004;16(8):1173-9.

Kaplan MH, Schindler U, Smiley ST, Grusby MJ. Stat6 is required for mediating responses to IL-4 and for development of Th2 cells. Immunity 1996a;4(3):313-9.

Kaplan MH, Sun YL, Hoey T, Grusby MJ. Impaired IL-12 responses and enhanced development of Th2 cells in Stat4-deficient mice. Nature 1996b;382(6587):174-7.

Kaptein A, Paillard V, Saunders M. Dominant negative Stat3 mutant inhibits interleukin-6-induced Jak-STAT signal transduction. J Biol Chem 1996;271(11):5961-4.

Kawai T, Akira S. The role of pattern-recognition receptors in innate immunity: update on Toll-like receptors. Nat Immunol 2010;11(5):373-84.

Kawata T, Shevchenko A, Fukuzawa M, Jermyn KA, Totty NF, Zhukovskaya NV, Sterling AE, Mann M, Williams JG. SH2 signaling in a lower eukaryote: a STAT protein that regulates stalk cell differentiation in dictyostelium. Cell 1997;89(6):909-16.

Kieseier BC, Hartung HP. Current disease-modifying therapies in multiple sclerosis. Semin Neurol 2003;23(2):133-46. 
Kikuchi S, Hata M, Fukumoto K, Yamane Y, Matsui T, Tamura A, Yonemura S, Yamagishi H, Keppler D, Tsukita S, Tsukita S. Radixin deficiency causes conjugated hyperbilirubinemia with loss of Mrp2 from bile canalicular membranes. Nat Genet 2002;31(3):320-5.

Killock DJ, Parsons M, Zarrouk M, Ameer-Beg SM, Ridley AJ, Haskard DO, Zvelebil M, Ivetic A. In vitro and in vivo characterization of molecular interactions between calmodulin, ezrin/radixin/moesin, and L-selectin. J Biol Chem 2009;284(13):8833-45.

Kingwell E, Marriott JJ, Jetté N, Pringsheim T, Makhani N, Morrow SA, Fisk JD, Evans C, Béland SG, Kulaga S, Dykeman J, Wolfson C, Koch MW, Marrie RA. Incidence and prevalence of multiple sclerosis in Europe: a systematic review. BMC Neurol 2013;13:128.

Knight RA, Scarabelli TM, Stephanou A. STAT transcription in the ischemic heart. JAKSTAT 2012;1(2):111-7.

Kono H, Rock KL. How dying cells alert the immune system to danger. Nat Rev Immunol 2008;8(4):279-89.

Korn T, Bettelli E, Gao W, Awasthi A, Jäger A, Strom TB, Oukka M, Kuchroo VK. IL-21 initiates an alternative pathway to induce proinflammatory T(H)17 cells. Nature 2007;448(7152):484-7.

Kotenko SV, Gallagher G, Baurin VV, Lewis-Antes A, Shen M, Shah NK, Langer JA, Sheikh F, Dickensheets H, Donnelly RP. IFN-lambdas mediate antiviral protection through a distinct class II cytokine receptor complex. Nat Immunol 2003;4(1):69-77.

Krakowski M, Owens T. Interferon-gamma confers resistance to experimental allergic encephalomyelitis. Eur J Immunol 1996;26(7):1641-6.

Krenger W, Falzarano G, Delmonte J Jr, Snyder KM, Byon JC, Ferrara JL. Interferon-gamma suppresses T-cell proliferation to mitogen via the nitric oxide pathway during experimental acute graft-versus-host disease. Blood 1996;88(3):1113-21.

Kroenke MA, Carlson TJ, Andjelkovic AV, Segal BM. IL-12- and IL-23-modulated T cells induce distinct types of EAE based on histology, CNS chemokine profile, and response to cytokine inhibition. J Exp Med 2008;205(7):1535-41.

Kurihara T, Warr G, Loy J, Bravo R. Defects in macrophage recruitment and host defense in mice lacking the CCR2 chemokine receptor. J Exp Med 1997;186(10):1757-62.

Lagresle-Peyrou C, Luce S, Ouchani F, Soheili TS, Sadek H, Chouteau M, Durand A, Pic I, Majewski J, Brouzes C, Lambert N, Bohineust A, Verhoeven E, Cosset FL, Magerus-Chatinet A, RieuxLaucat F, Gandemer V, Monnier D, Heijmans C, van Gijn M, Dalm VA, Mahlaoui N, Stephan JL, Picard C, Durandy A, Kracker S, Hivroz C, Jabado N, de Saint Basile G, Fischer A, Cavazzana M, André-Schmutz I. X-linked primary immunodeficiency associated with hemizygous mutations in the moesin (MSN) gene. J Allergy Clin Immunol 2016 S0091-6749(16)30423-7.

Lamb RF, Ozanne BW, Roy C, McGarry L, Stipp C, Mangeat P, Jay DG. Essential functions of ezrin in maintenance of cell shape and lamellipodial extension in normal and transformed fibroblasts. Curr Biol 1997;7(9):682-8.

Langer JA, Pestka S. Interferon receptors. Immunol Today 1988;9(12):393-400.

Larner AC, David M, Feldman GM, Igarashi K, Hackett RH, Webb DS, Sweitzer SM, Petricoin EF 3rd, Finbloom DS. Tyrosine phosphorylation of DNA binding proteins by multiple cytokines. Science 1993;261(5129):1730-3. 
Lazear HM, Nice TJ, Diamond MS. Interferon-lambda: immune functions at barrier surfaces and beyond. Immunity 2015;43(1):15-28.

Lee J, Katakai T, Hara T, Gonda H, Sugai M, Shimizu A. Roles of p-ERM and Rho-ROCK signaling in lymphocyte polarity and uropod formation. J Cell Biol 2004;167(2):327-37.

Leiphrakpam PD, Rajput A, Mathiesen M, Agarwal E, Lazenby AJ, Are C, Brattain MG, Chowdhury S. Ezrin expression and cell survival regulation in colorectal cancer. Cell Signal 2014;26(5):86879.

Leung $\mathrm{S}$, Li X, Stark GR. STATs find that hanging together can be stimulating. Science 1996;273(5276):750-1.

Levy DE, Darnell JE Jr. Stats: transcriptional control and biological impact. Nat Rev Mol Cell Biol 2002;3(9):651-62.

Li XL, Lv J, Xi NN, Wang T, Shang XF, Xu HQ, Han Z, O'Byrne KT, Li XF, Zheng RY. Neonatal endotoxin exposure suppresses experimental autoimmune encephalomyelitis through regulating the immune cells responsivity in the central nervous system of adult rats. Biochem Biophys Res Commun 2010;398(2):302-8.

Li Y, Harada T, Juang YT, Kyttaris VC, Wang Y, Zidanic M, Tung K, Tsokos GC. Phosphorylated ERM is responsible for increased $\mathrm{T}$ cell polarization, adhesion, and migration in patients with systemic lupus erythematosus. J Immunol 2007;178(3):1938-47.

Lighvani AA, Frucht DM, Jankovic D, Yamane H, Aliberti J, Hissong BD, Nguyen BV, Gadina M, Sher A, Paul WE, O'Shea JJ. T-bet is rapidly induced by interferon-gamma in lymphoid and myeloid cells. Proc Natl Acad Sci U S A 2001;98(26):15137-42.

Lin JX, Li P, Liu D, Jin HT, He J, Ata Ur Rasheed M, Rochman Y, Wang L, Cui K, Liu C, Kelsall BL, Ahmed R, Leonard WJ. Critical role of STAT5 transcription factor tetramerization for cytokine responses and normal immune function. Immunity 2012;36(4):586-99.

Linossi EM, Nicholson SE. Kinase inhibition, competitive binding and proteasomal degradation: resolving the molecular function of the suppressor of cytokine signaling (SOCS) proteins. Immunol Rev 2015;266(1):123-33.

Liu B, Mink S, Wong KA, Stein N, Getman C, Dempsey PW, Wu H, Shuai K. PIAS1 selectively inhibits interferon-inducible genes and is important in innate immunity. Nat Immunol 2004;5(9):891-8.

Liu X, Robinson GW, Wagner KU, Garrett L, Wynshaw-Boris A, Hennighausen L. Stat5a is mandatory for adult mammary gland development and lactogenesis. Genes Dev 1997;11(2):17986.

Livak KJ, Schmittgen TD. Analysis of relative gene expression data using real-time quantitative PCR and the 2(-Delta Delta C(T)) Method. Methods 2001;25(4):402-8.

Lödige I, Marg A, Wiesner B, Malecová B, Oelgeschläger T, Vinkemeier U. Nuclear export determines the cytokine sensitivity of STAT transcription factors. $J$ Biol Chem 2005;280(52):43087-99.

Ma CS, Wong N, Rao G, Nguyen A, Avery DT, Payne K, Torpy J, O'Young P, Deenick E, Bustamante J, Puel A, Okada S, Kobayashi M, Martinez-Barricarte R, Elliott M, Sebnem Kilic S, El Baghdadi J, Minegishi Y, Bousfiha A, Robertson N, Hambleton S, Arkwright PD, French M, 
Blincoe AK, Hsu P, Campbell DE, Stormon MO, Wong M, Adelstein S, Fulcher DA, Cook MC, Stepensky P, Boztug K, Beier R, Ikincioğullari A, Ziegler JB, Gray P, Picard C, BoissonDupuis S, Phan TG, Grimbacher B, Warnatz K, Holland SM, Uzel G, Casanova JL, Tangye SG. Unique and shared signaling pathways cooperate to regulate the differentiation of human CD4+ $\mathrm{T}$ cells into distinct effector subsets. J Exp Med 2016 213(8):1589-608.

Majoros A, Platanitis E, Szappanos D, Cheon H, Vogl C, Shukla P, Stark GR, Sexl V, Schreiber R, Schindler C, Müller M, Decker T. Response to interferons and antibacterial innate immunity in the absence of tyrosine-phosphorylated STAT1. EMBO Rep 2016;17(3):367-82.

Mao X, Ren Z, Parker GN, Sondermann H, Pastorello MA, Wang W, McMurray JS, Demeler B, Darnell JE Jr, Chen X. Structural bases of unphosphorylated STAT1 association and receptor binding. Mol Cell 2005;17(6):761-71.

Marcelo P, Lefèvre F. Induction of the IFN-gamma gene and protein is linked to the establishment of cell polarity in a porcine epithelial cell line. Exp Cell Res 2002;280(1):33-44.

Matsui T, Maeda M, Doi Y, Yonemura S, Amano M, Kaibuchi K, Tsukita S, Tsukita S. Rho-kinase phosphorylates $\mathrm{COOH}$-terminal threonines of ezrin/radixin/moesin (ERM) proteins and regulates their head-to-tail association. J Cell Biol 1998;140(3):647-57.

Matsumoto M, Hirata T. Moesin regulates neutrophil rolling velocity in vivo. Cell Immunol 2016;304305:59-62.

McBride KM, Banninger G, McDonald C, Reich NC. Regulated nuclear import of the STAT1 transcription factor by direct binding of importin-alpha. EMBO J 2002;21(7):1754-63.

McCormick J, Barry SP, Sivarajah A, Stefanutti G, Townsend PA, Lawrence KM, Eaton S, Knight RA, Thiemermann C, Latchman DS, Stephanou A. Free radical scavenging inhibits STAT phosphorylation following in vivo ischemia/reperfusion injury. FASEB J 2006;20(12):2115-7.

McCormick J, Suleman N, Scarabelli TM, Knight RA, Latchman DS, Stephanou A. STAT1 deficiency in the heart protects against myocardial infarction by enhancing autophagy. J Cell Mol Med 2012;16(2):386-93.

McKendry R, John J, Flavell D, Müller M, Kerr IM, Stark GR. High-frequency mutagenesis of human cells and characterization of a mutant unresponsive to both alpha and gamma interferons. Proc Natl Acad Sci U S A 1991;88(24):11455-9.

McWhorter FY, Wang T, Nguyen P, Chung T, Liu WF. Modulation of macrophage phenotype by cell shape. Proc Natl Acad Sci U S A 2013;110(43):17253-8.

Menager C, Vassy J, Doliger C, Legrand Y, Karniguian A. Subcellular localization of RhoA and ezrin at membrane ruffles of human endothelial cells: differential role of collagen and fibronectin. Exp Cell Res 1999;249(2):221-30.

Meraz MA, White JM, Sheehan KC, Bach EA, Rodig SJ, Dighe AS, Kaplan DH, Riley JK, Greenlund AC, Campbell D, Carver-Moore K, DuBois RN, Clark R, Aguet M, Schreiber RD. Targeted disruption of the Stat1 gene in mice reveals unexpected physiologic specificity in the JAK-STAT signaling pathway. Cell 1996;84(3):431-42.

Mertens C, Haripal B, Klinge S, Darnell JE Jr. Mutations in the linker domain affect phospho-STAT3 function and suggest targets for interrupting STAT3 activity. Proc Natl Acad Sci U S A 2015;112(48):14811-6. 
Mertens C, Zhong M, Krishnaraj R, Zou W, Chen X, Darnell JE Jr. Dephosphorylation of phosphotyrosine on STAT1 dimers requires extensive spatial reorientation of the monomers facilitated by the N-terminal domain. Genes Dev 2006;20(24):3372-81.

Meyer T, Begitt A, Lödige I, van Rossum M, Vinkemeier U. Constitutive and IFN-gamma-induced nuclear import of STAT1 proceed through independent pathways. EMBO J 2002;21(3):344-54.

Meyer T, Begitt A, Vinkemeier U. Green fluorescent protein-tagging reduces the nucleocytoplasmic shuttling specifically of unphosphorylated STAT1. FEBS J 2007;274(3):815-26.

Meyer T, Hendry L, Begitt A, John S, Vinkemeier U. A single residue modulates tyrosine dephosphorylation, oligomerization, and nuclear accumulation of stat transcription factors. J Biol Chem 2004;279(18):18998-9007.

Meyer T, Marg A, Lemke P, Wiesner B, Vinkemeier U. DNA binding controls inactivation and nuclear accumulation of the transcription factor Stat1. Genes Dev 2003;17(16):1992-2005.

Moriggl R, Topham DJ, Teglund S, Sexl V, McKay C, Wang D, Hoffmeyer A, van Deursen J, Sangster MY, Bunting KD, Grosveld GC, Ihle JN. Stat5 is required for IL-2-induced cell cycle progression of peripheral T cells. Immunity 1999;10(2):249-59.

Mosmann TR, Cherwinski H, Bond MW, Giedlin MA, Coffman RL. Two types of murine helper T cell clone. I. Definition according to profiles of lymphokine activities and secreted proteins. J Immunol 1986;136(7):2348-57.

Murphy TL, Geissal ED, Farrar JD, Murphy KM. Role of the Stat4 N domain in receptor proximal tyrosine phosphorylation. Mol Cell Biol 2000;20(19):7121-31.

Nefedova Y, Huang M, Kusmartsev S, Bhattacharya R, Cheng P, Salup R, Jove R, Gabrilovich D. Hyperactivation of STAT3 is involved in abnormal differentiation of dendritic cells in cancer. J Immunol 2004;172(1):464-74.

Niggli V. Insights into the mechanism for dictating polarity in migrating T-cells. Int Rev Cell Mol Biol 2014;312:201-70.

Nishibori T, Tanabe Y, Su L, David M. Impaired development of CD4+ CD25+ regulatory T cells in the absence of STAT1: increased susceptibility to autoimmune disease. $J$ Exp Med 2004;199(1):25-34.

Nogai A, Siffrin V, Bonhagen K, Pfueller CF, Hohnstein T, Volkmer-Engert R, Brück W, Stadelmann C, Kamradt T. Lipopolysaccharide injection induces relapses of experimental autoimmune encephalomyelitis in nontransgenic mice via bystander activation of autoreactive CD4+ cells. J Immunol 2005;175(2):959-66.

Novick D, Cohen B, Rubinstein M. The human interferon alpha/beta receptor: characterization and molecular cloning. Cell 1994;77(3):391-400.

Okamoto I, Kawano Y, Matsumoto M, Suga M, Kaibuchi K, Ando M, Saya H. Regulated CD44 cleavage under the control of protein kinase $\mathrm{C}$, calcium influx, and the Rho family of small $\mathrm{G}$ proteins. J Biol Chem 1999;274(36):25525-34.

Ouadrhiri Y, Sibille Y, Tulkens PM. Modulation of intracellular growth of Listeria monocytogenes in human enterocyte Caco-2 cells by interferon-gamma and interleukin-6: role of nitric oxide and cooperation with antibiotics. J Infect Dis 1999;180(4):1195-204. 
Park C, Li S, Cha E, Schindler C. Immune response in Stat2 knockout mice. Immunity 2000;13(6):795-804.

Park H, Li Z, Yang XO, Chang SH, Nurieva R, Wang YH, Wang Y, Hood L, Zhu Z, Tian Q, Dong C. A distinct lineage of CD4 T cells regulates tissue inflammation by producing interleukin 17. Nat Immunol 2005;6(11):1133-41.

Park JH, Jeong SY, Choi AJ, Kim SJ. Lipopolysaccharide directly stimulates Th17 differentiation in vitro modulating phosphorylation of RelB and NF-kappaB1. Immunol Lett 2015;165(1):10-9.

Pérez-Rivero G, Cascio G, Soriano SF, Sanz ÁG, Guinoa JS de, Rodríguez-Frade JM, Gomariz RP, Holgado BL, Cabañas C, Carrasco YR, Stein JV, Mellado M. Janus kinases 1 and 2 regulate chemokine-mediated integrin activation and naive T-cell homing. Eur $J$ Immunol 2013;43(7):1745-57.

Pestonjamasp K, Amieva MR, Strassel CP, Nauseef WM, Furthmayr H, Luna EJ. Moesin, ezrin, and p205 are actin-binding proteins associated with neutrophil plasma membranes. Mol Biol Cell 1995;6(3):247-59.

Peters A, Fowler KD, Chalmin F, Merkler D, Kuchroo VK, Pot C. IL-27 induces Th17 differentiation in the absence of STAT1 signaling. J Immunol 2015;195(9):4144-53.

Piro M, Della Bona R, Abbate A, Biasucci LM, Crea F. Sex-related differences in myocardial remodeling. J Am Coll Cardiol 2010;55(11):1057-65.

Platanias LC. Mechanisms of type-I- and type-II-interferon-mediated signalling. Nat Rev Immunol 2005;5(5):375-86.

Pore D, Gupta N. The ezrin-radixin-moesin family of proteins in the regulation of B-cell immune response. Crit Rev Immunol 2015;35(1):15-31.

Potter DA, Tirnauer JS, Janssen R, Croall DE, Hughes CN, Fiacco KA, Mier JW, Maki M, Herman IM. Calpain regulates actin remodeling during cell spreading. J Cell Biol 1998;141(3):647-62.

Puddu P, Fais S, Luciani F, Gherardi G, Dupuis ML, Romagnoli G, Ramoni C, Cianfriglia M, Gessani S. Interferon-gamma up-regulates expression and activity of P-glycoprotein in human peripheral blood monocyte-derived macrophages. Lab Invest 1999;79(10):1299-309.

Qing Y, Stark GR. Alternative activation of STAT1 and STAT3 in response to interferon-gamma. J Biol Chem 2004;279(40):41679-85.

Rangachari M, Kuchroo VK. Using EAE to better understand principles of immune function and autoimmune pathology. J Autoimmun 2013;45:31-9.

Rawlings JS, Rosler KM, Harrison DA. The JAK/STAT signaling pathway. J Cell Sci 2004;117(Pt 8):1281-3.

Raziuddin S, Morrison DC. Prolonged suppression of experimental allergic encephalomyelitis in the Lewis rats: the effect of lipopolysaccharides. J Immunol 1982;128(3):1486-9.

Refaeli Y, van Parijs L, Alexander SI, Abbas AK. Interferon gamma is required for activation-induced death of T lymphocytes. J Exp Med 2002;196(7):999-1005.

Riley JK, Takeda K, Akira S, Schreiber RD. Interleukin-10 receptor signaling through the JAK-STAT pathway. Requirement for two distinct receptor-derived signals for anti-inflammatory action. J Biol Chem 1999;274(23):16513-21. 
Sahrbacher UC, Lechner F, Eugster HP, Frei K, Lassmann H, Fontana A. Mice with an inactivation of the inducible nitric oxide synthase gene are susceptible to experimental autoimmune encephalomyelitis. Eur J Immunol 1998;28(4):1332-8.

Sauvé M, Ban K, Momen MA, Zhou YQ, Henkelman RM, Husain M, Drucker DJ. Genetic deletion or pharmacological inhibition of dipeptidyl peptidase-4 improves cardiovascular outcomes after myocardial infarction in mice. Diabetes 2010;59(4):1063-73.

Saygideğer-Kont Y, Minas TZ, Jones H, Hour S, Çelik H, Temel I, Han J, Atabey N, Erkizan HV, Toretsky JA, Üren A. Ezrin enhances EGFR signaling and modulates Erlotinib sensitivity in nonsmall cell lung cancer cells. Neoplasia 2016;18(2):111-20.

Scaffidi P, Misteli T, Bianchi ME. Release of chromatin protein HMGB1 by necrotic cells triggers inflammation. Nature 2002;418(6894):191-5.

Scarabelli TM, Gottlieb RA. Functional and clinical repercussions of myocyte apoptosis in the multifaceted damage by ischemia/reperfusion injury: old and new concepts after 10 years of contributions. Cell Death Differ 2004;11 Suppl 2:S144-52.

Scarabelli TM, Mariotto S, Abdel-Azeim S, Shoji K, Darra E, Stephanou A, Chen-Scarabelli C, Marechal JD, Knight R, Ciampa A, Saravolatz L, de Prati AC, Yuan Z, Cavalieri E, Menegazzi M, Latchman D, Pizza C, Perahia D, Suzuki H. Targeting STAT1 by myricetin and delphinidin provides efficient protection of the heart from ischemia/reperfusion-induced injury. FEBS Lett 2009;583(3):531-41.

Schaefer TS, Sanders LK, Nathans D. Cooperative transcriptional activity of Jun and Stat3 beta, a short form of Stat3. Proc Natl Acad Sci U S A 1995;92(20):9097-101.

Scherer WF, Syverton JT, Gey GO. Studies on the propagation in vitro of poliomyelitis viruses IV. Viral mltiplication in a stable strain of human malignant epithelial cells (strain HeLa) derived from an epidermoid carcinoma of the cervix. J Exp Med 1953(97(5)):695-710.

Scherrer-Crosbie M, Ullrich R, Bloch KD, Nakajima H, Nasseri B, Aretz HT, Lindsey ML, Vançon AC, Huang PL, Lee RT, Zapol WM, Picard MH. Endothelial nitric oxide synthase limits left ventricular remodeling after myocardial infarction in mice. Circulation 2001;104(11):128691.

Schindelin J, Arganda-Carreras I, Frise E, Kaynig V, Longair M, Pietzsch T, Preibisch S, Rueden C, Saalfeld S, Schmid B, Tinevez JY, White DJ, Hartenstein V, Eliceiri K, Tomacak P, Cardona A. Fiji: an open-source platform for biological-image analysis. Nat Methods 2012;9(7):676-82.

Schindler C, Fu XY, Improta T, Aebersold R, Darnell JE Jr. Proteins of transcription factor ISGF-3: one gene encodes the 91 -and $84-\mathrm{kDa}$ ISGF-3 proteins that are activated by interferon alpha. Proc Natl Acad Sci U S A 1992a;89(16):7836-9.

Schindler C, Shuai K, Prezioso VR, Darnell JE Jr. Interferon-dependent tyrosine phosphorylation of a latent cytoplasmic transcription factor. Science 1992b;257(5071):809-13.

Schneider CA, Rasband WS, Eliceiri KW. NIH Image to ImageJ: 25 years of image analysis. Nat Methods 2012;9(7):671-5.

Sheppard P, Kindsvogel W, Xu W, Henderson K, Schlutsmeyer S, Whitmore TE, Kuestner R, Garrigues U, Birks C, Roraback J, Ostrander C, Dong D, Shin J, Presnell S, Fox B, Haldeman B, Cooper E, Taft D, Gilbert T, Grant FJ, Tackett M, Krivan W, McKnight G, Clegg C, Foster D, 
Klucher KM. IL-28, IL-29 and their class II cytokine receptor IL-28R. Nat Immunol 2003;4(1):638.

Shimoda K, van Deursen J, Sangster MY, Sarawar SR, Carson RT, Tripp RA, Chu C, Quelle FW, Nosaka T, Vignali DA, Doherty PC, Grosveld G, Paul WE, Ihle JN. Lack of IL-4-induced Th2 response and IgE class switching in mice with disrupted Stat6 gene. Nature 1996;380(6575):630 3.

Shuai K. Modulation of STAT signaling by STAT-interacting proteins. Oncogene 2000;19(21):263844.

Shuai K. Regulation of cytokine signaling pathways by PIAS proteins. Cell Res 2006;16(2):196-202.

Shuai K, Schindler C, Prezioso VR, Darnell JE Jr. Activation of transcription by IFN-gamma: tyrosine phosphorylation of a 91-kD DNA binding protein. Science 1992;258(5089):1808-12.

Shuai K, Stark GR, Kerr IM, Darnell JE Jr. A single phosphotyrosine residue of Stat91 required for gene activation by interferon-gamma. Science 1993a;261(5129):1744-6.

Shuai K, Ziemiecki A, Wilks AF, Harpur AG, Sadowski HB, Gilman MZ, Darnell JE Jr. Polypeptide signalling to the nucleus through tyrosine phosphorylation of Jak and Stat proteins. Nature 1993b;366(6455):580-3.

Slavin AJ, Soos JM, Stuve O, Patarroyo JC, Weiner HL, Fontana A, Bikoff EK, Zamvil SS. Requirement for endocytic antigen processing and influence of invariant chain and $\mathrm{H}-2 \mathrm{M}$ deficiencies in CNS autoimmunity. J Clin Invest 2001;108(8):1133-9.

Soulika AM, Lee E, McCauley E, Miers L, Bannerman P, Pleasure D. Initiation and progression of axonopathy in experimental autoimmune encephalomyelitis. J Neurosci 2009;29(47):14965-79.

Speil J, Baumgart E, Siebrasse JP, Veith R, Vinkemeier U, Kubitscheck U. Activated STAT1 transcription factors conduct distinct saltatory movements in the cell nucleus. Biophys $J$ 2011;101(11):2592-600.

Staab J, Riebeling T, Koch V, Herrmann-Lingen C, Meyer T. The two interfaces of the STAT1 Nterminus exhibit opposite functions in IFNgamma-regulated gene expression. Mol Immunol 2015;67(2 Pt B):596-606.

Stark GR. How cells respond to interferons revisited: from early history to current complexity. Cytokine Growth Factor Rev 2007;18(5-6):419-23.

Starr R, Hilton DJ. Negative regulation of the JAK/STAT pathway. Bioessays 1999;21(1):47-52.

Stephanou A, Brar BK, Scarabelli TM, Jonassen AK, Yellon DM, Marber MS, Knight RA, Latchman DS. J Biol Chem 2000;275(14):10002-8.

Stephanou A, Scarabelli TM, Townsend PA, Bell R, Yellon D, Knight RA, Latchman DS. The carboxyl-terminal activation domain of the STAT-1 transcription factor enhances ischemia/reperfusion-induced apoptosis in cardiac myocytes. FASEB $J$ 2002;16(13):1841-3.

Strehlow I, Schindler C. Amino-terminal signal transducer and activator of transcription (STAT) domains regulate nuclear translocation and STAT deactivation. J Biol Chem 1998;273(43):2804956 .

Stritesky GL, Yeh N, Kaplan MH. IL-23 promotes maintenance but not commitment to the Th17 lineage. J Immunol 2008;181(9):5948-55. 
Su SB, Grajewski RS, Luger D, Agarwal RK, Silver PB, Tang J, Tuo J, Chan CC, Caspi RR. Altered chemokine profile associated with exacerbated autoimmune pathology under conditions of genetic interferon-gamma deficiency. Invest Ophthalmol Vis Sci 2007;48(10):4616-25.

Surin B, Rouillard D, Bauvois B. Loss of alpha5beta1-mediated adhesion of monocytic cells to fibronectin by interferons beta and gamma is associated with changes in actin and paxillin cytoskeleton. Hematol J 2000;1(3):172-80.

Takeda K, Akira S. STAT family of transcription factors in cytokine-mediated biological responses. Cytokine Growth Factor Rev 2000;11(3):199-207.

Takeda K, Kishimoto T, Akira S. STAT6: its role in interleukin 4-mediated biological functions. J Mol Med (Berl) 1997a;75(5):317-26.

Takeda K, Noguchi K, Shi W, Tanaka T, Matsumoto M, Yoshida N, Kishimoto T, Akira S. Targeted disruption of the mouse Stat3 gene leads to early embryonic lethality. Proc Natl Acad Sci U S A 1997b;94(8):3801-4.

Takeuchi K, Sato N, Kasahara H, Funayama N, Nagafuchi A, Yonemura S, Tsukita S, Tsukita S. Perturbation of cell adhesion and microvilli formation by antisense oligonucleotides to ERM family members. J Cell Biol 1994;125(6):1371-84.

Tamura A, Kikuchi S, Hata M, Katsuno T, Matsui T, Hayashi H, Suzuki Y, Noda T, Tsukita S, Tsukita S. Achlorhydria by ezrin knockdown: defects in the formation/expansion of apical canaliculi in gastric parietal cells. J Cell Biol 2005;169(1):21-8.

Tojo SJ, Yokota S, Koike H, Schultz J, Hamazume Y, Misugi E, Yamada K, Hayashi M, Paulson JC, Morooka S. Reduction of rat myocardial ischemia and reperfusion injury by sialyl Lewis $\mathrm{x}$ oligosaccharide and anti-rat P-selectin antibodies. Glycobiology 1996;6(4):463-9.

Tran EH, Prince EN, Owens T. IFN-gamma shapes immune invasion of the central nervous system via regulation of chemokines. J Immunol 2000;164(5):2759-68.

Udy GB, Towers RP, Snell RG, Wilkins RJ, Park SH, Ram PA, Waxman DJ, Davey HW. Requirement of STAT5b for sexual dimorphism of body growth rates and liver gene expression. Proc Natl Acad Sci U S A 1997;94(14):7239-44.

Utech M, Ivanov AI, Samarin SN, Bruewer M, Turner JR, Mrsny RJ, Parkos CA, Nusrat A. Mechanism of IFN-gamma-induced endocytosis of tight junction proteins: myosin II-dependent vacuolarization of the apical plasma membrane. Mol Biol Cell 2005;16(10):5040-52.

Valitutti S, Dessing M, Aktories K, Gallati H, Lanzavecchia A. Sustained signaling leading to T cell activation results from prolonged $\mathrm{T}$ cell receptor occupancy. Role of $\mathrm{T}$ cell actin cytoskeleton. J Exp Med 1995;181(2):577-84.

van der Veen RC, Dietlin TA, Pen L, Gray JD, Hofman FM. Antigen presentation to Th1 but not Th2 cells by macrophages results in nitric oxide production and inhibition of $\mathrm{T}$ cell proliferation: interferon-gamma is essential but insufficient. Cell Immunol 2000;206(2):125-35.

Villarino AV, Gallo E, Abbas AK. STAT1-activating cytokines limit Th17 responses through both Tbet-dependent and -independent mechanisms. J Immunol 2010;185(11):6461-71.

Vilmos P, Kristó I, Szikora S, Jankovics F, Lukácsovich T, Kari B, Erdélyi M. The actin-binding ERM protein moesin directly regulates spindle assembly and function during mitosis. Cell Biol Int 2016;40(6):696-707. 
Vinkemeier U, Cohen SL, Moarefi I, Chait BT, Kuriyan J, Darnell JE Jr. DNA binding of in vitro activated Stat1 alpha, Stat1 beta and truncated Stat1: interaction between NH2-terminal domains stabilizes binding of two dimers to tandem DNA sites. EMBO J 1996;15(20):5616-26.

Vinkemeier U, Moarefi I, Darnell JE Jr, Kuriyan J. Structure of the amino-terminal protein interaction domain of STAT-4. Science 1998;279(5353):1048-52.

Wan CK, Andraski AB, Spolski R, Li P, Kazemian M, Oh J, Samsel L, Swanson PA 2nd, McGavern DB, Sampaio EP, Freeman AF, Milner JD, Holland SM, Leonard WJ. Opposing roles of STAT1 and STAT3 in IL-21 function in CD4+ T cells. Proc Natl Acad Sci U S A 2015;112(30):9394-9.

Wang C, Morley SC, Donermeyer D, Peng I, Lee WP, Devoss J, Danilenko DM, Lin Z, Zhang J, Zhou J, Allen PM, Brown EJ. Actin-bundling protein L-plastin regulates T cell activation. J Immunol 2010;185(12):7487-97.

Wang D, Stravopodis D, Teglund S, Kitazawa J, Ihle JN. Naturally occurring dominant negative variants of Stat5. Mol Cell Biol 1996;16(11):6141-8.

Wang Y, Yago T, Zhang N, Abdisalaam S, Alexandrakis G, Rodgers W, McEver RP. Cytoskeletal regulation of CD44 membrane organization and interactions with E-selectin. J Biol Chem 2014;289(51):35159-71.

Wang Z, Hong J, Sun W, Xu G, Li N, Chen X, Liu A, Xu L, Sun B, Zhang JZ. Role of IFN-gamma in induction of Foxp3 and conversion of CD4+ CD25- T cells to CD4+ Tregs. J Clin Invest 2006;116(9):2434-41.

Watanabe K, Tachibana M, Kim S, Watarai M. Participation of ezrin in bacterial uptake by trophoblast giant cells. Reprod Biol Endocrinol 2009;7:95.

Webb JL, Harvey MW, Holden DW, Evans TJ. Macrophage nitric oxide synthase associates with cortical actin but is not recruited to phagosomes. Infect Immun 2001;69(10):6391-400.

Wegenka UM, Buschmann J, Lütticken C, Heinrich PC, Horn F. Acute-phase response factor, a nuclear factor binding to acute-phase response elements, is rapidly activated by interleukin- 6 at the posttranslational level. Mol Cell Biol 1993;13(1):276-88.

Wegrzyn J, Potla R, Chwae YJ, Sepuri NB, Zhang Q, Koeck T, Drecka M, Szczepanek K, Szelag M, Gornicka A, Moh A, Moghaddas S, Chen Q, Bobbili S, Cichy J, Dulak J, Baker DP, Wolfman A, Stuehr D, Hassan MO, Fu XY, Avadhani N, Drake JI, Fawcett P, Lesnefsky EJ, Larner AC. Function of mitochondrial Stat3 in cellular respiration. Science 2009;323(5915):793-7.

Wenta N, Strauss H, Meyer S, Vinkemeier U. Tyrosine phosphorylation regulates the partitioning of STAT1 between different dimer conformations. Proc Natl Acad Sci U S A 2008;105(27):9238-43.

White HD, Norris RM, Brown MA, Brandt PW, Whitlock RM, Wild CJ. Left ventricular end-systolic volume as the major determinant of survival after recovery from myocardial infarction. Circulation 1987;76(1):44-51.

Willenborg DO, Fordham S, Bernard CC, Cowden WB, Ramshaw IA. IFN-gamma plays a critical down-regulatory role in the induction and effector phase of myelin oligodendrocyte glycoproteininduced autoimmune encephalomyelitis. J Immunol 1996;157(8):3223-7.

Willenborg DO, Fordham SA, Staykova MA, Ramshaw IA, Cowden WB. IFN-gamma is critical to the control of murine autoimmune encephalomyelitis and regulates both in the periphery and in the target tissue: a possible role for nitric oxide. J Immunol 1999;163(10):5278-86. 
Wurster AL, Tanaka T, Grusby MJ. The biology of Stat4 and Stat6. Oncogene 2000;19(21):2577-84.

Xiang FL, Lu X, Hammoud L, Zhu P, Chidiac P, Robbins J, Feng Q. Cardiomyocyte-specific overexpression of human stem cell factor improves cardiac function and survival after myocardial infarction in mice. Circulation 2009;120(12):1065-74, 9 p following 1074.

Yamaoka K, Saharinen P, Pesu M, HoltVE 3rd, Silvennoinen O, O'Shea JJ. The Janus kinases (Jaks). Genome Biol 2004;5(12):253.

Yamazaki T, Seko Y, Tamatani T, Miyasaka M, Yagita H, Okumura K, Nagai R, Yazaki Y. Expression of intercellular adhesion molecule-1 in rat heart with ischemia/reperfusion and limitation of infarct size by treatment with antibodies against cell adhesion molecules. Am J Pathol 1993;143(2):410-8.

Yan R, Small S, Desplan C, Dearolf CR, Darnell JE Jr. Identification of a Stat gene that functions in Drosophila development. Cell 1996;84(3):421-30.

Yang E, Henriksen MA, Schaefer O, Zakharova N, Darnell JE Jr. Dissociation time from DNA determines transcriptional function in a STAT1 linker mutant. J Biol Chem 2002;277(16):1345562 .

Yang J, Stark GR. Roles of unphosphorylated STATs in signaling. Cell Res 2008;18(4):443-51.

Yang XO, Panopoulos AD, Nurieva R, Chang SH, Wang D, Watowich SS, Dong C. STAT3 regulates cytokine-mediated generation of inflammatory helper T cells. J Biol Chem 2007;282(13):9358-63.

Yao X, Thibodeau A, Forte JG. Ezrin-calpain I interactions in gastric parietal cells. Am J Physiol 1993;265(1 Pt 1):C36-46.

Yeh WI, McWilliams IL, Harrington LE. Autoreactive Tbet-positive CD4 T cells develop independent of classic Th1 cytokine signaling during experimental autoimmune encephalomyelitis. J Immunol 2011;187(10):4998-5006.

Youakim A, Ahdieh M. Interferon-gamma decreases barrier function in T84 cells by reducing ZO-1 levels and disrupting apical actin. Am J Physiol 1999;276(5 Pt 1):G1279-88.

Zawawi KH, Kantarci A, Schulze-Späte U, Fujita T, Batista EL Jr, Amar S, van Dyke TE. Moesininduced signaling in response to lipopolysaccharide in macrophages. J Periodontal Res 2010;45(5):589-601.

Zettl UK, Mix E, Zielasek J, Stangel M, Hartung HP, Gold R. Apoptosis of myelin-reactive T cells induced by reactive oxygen and nitrogen intermediates in vitro. Cell Immunol 1997;178(1):1-8.

Zhang SY, Boisson-Dupuis S, Chapgier A, Yang K, Bustamante J, Puel A, Picard C, Abel L, Jouanguy E, Casanova JL. Inborn errors of interferon (IFN)-mediated immunity in humans: insights into the respective roles of IFN-alpha/beta, IFN-gamma, and IFN-lambda in host defense. Immunol Rev 2008;226:29-40.

Zhang Y, Hu MY, Wu WZ, Wang ZJ, Zhou K, Zha XL, Liu KD. The membrane-cytoskeleton organizer ezrin is necessary for hepatocellular carcinoma cell growth and invasiveness. $J$ Cancer Res Clin Oncol 2006;132(11):685-97.

Zheng SG, Wang JH, Gray JD, Soucier H, Horwitz DA. Natural and induced CD4+CD25+ cells educate CD4+CD25- cells to develop suppressive activity: the role of IL-2, TGF-beta, and IL-10. J Immunol 2004;172(9):5213-21. 
Zhong M, Henriksen MA, Takeuchi K, Schaefer O, Liu B, Hoeve J ten, Ren Z, Mao X, Chen X, Shuai K, Darnell JE Jr. Implications of an antiparallel dimeric structure of nonphosphorylated STAT1 for the activation-inactivation cycle. Proc Natl Acad Sci U S A 2005;102(11):3966-71.

Zhou H, Wang Y, Lian Q, Yang B, Ma Y, Wu X, Sun S, Liu Y, Sun B. Differential IL-10 production by DCs determines the distinct adjuvant effects of LPS and PTX in EAE induction. Eur J Immunol 2014;44(5):1352-62.

Zouein FA, Altara R, Chen Q, Lesnefsky EJ, Kurdi M, Booz GW. Pivotal importance of STAT3 in protecting the heart from acute and chronic stress: new advancement and unresolved issues. Front Cardiovasc Med 2015;2:36. 


\section{Curriculum vitae}

Name

Date of Birth

Place of Birth

Nationality

\section{Education:}

05/2013 - present

$10 / 2011-04 / 2013$

$10 / 2008-08 / 2011$

$08 / 1999-06 / 2008$
Theresa Riebeling

8 September 1988

Schwalmstadt, Germany

German
Doctorate student, Institute of Psychosomatic Medicine and Psychotherapy, Department of Molecular Psychocardiology, University Medical Centre Göttingen, Germany, $\mathrm{PhD}$ program "Molecular Medicine"

Research project 10 of the IRTG1816

Master student, Molecular Medicine, Georg-AugustUniversity, Göttingen, Germany,

Master thesis: Oxidative stress as a potential cause for residual DNA damage and adverse side effects of radiation therapy in cancer patients; Grade: 1.5

Bachelor student, Molecular Medicine, Georg-AugustUniversity, Göttingen, Germany, Bachelor thesis: Studies on the development of hypertrophic cardiomyopathy in Hspa4deficient mice; Grade: 1.75

Abitur, Melanchthon-Schule, Willingshausen-Steinatal, Germany; Grade 1.4

\section{Participation in conferences:}

29 May - 03 June 2016: Gordon Research Conference, 'Chemotactic Cytokines', Girona, Spain, poster presentation: Transription of the cytoskeletal protein ezrin is induced by interferon- $\gamma$-activated STAT1

15 - 16 September 2014: Joint KCL-JCI International Symposium, 'Recent advances in heart failure', London, UK, poster presentation: Binding to High-Affinity DNA Sequences Prevents the Disruption of STAT1 dimers 